\title{
Exploration of the sustainability of innovations in hospital care
}

Citation for published version (APA):

Ament, S. M. C. (2015). Exploration of the sustainability of innovations in hospital care. [Doctoral Thesis, Maastricht University]. Datawyse / Universitaire Pers Maastricht. https://doi.org/10.26481/dis.20150904sa

Document status and date:

Published: 01/01/2015

DOI:

10.26481/dis.20150904sa

Document Version:

Publisher's PDF, also known as Version of record

\section{Please check the document version of this publication:}

- A submitted manuscript is the version of the article upon submission and before peer-review. There can be important differences between the submitted version and the official published version of record.

People interested in the research are advised to contact the author for the final version of the publication, or visit the DOI to the publisher's website.

- The final author version and the galley proof are versions of the publication after peer review.

- The final published version features the final layout of the paper including the volume, issue and page numbers.

Link to publication

\footnotetext{
General rights rights.

- You may freely distribute the URL identifying the publication in the public portal. please follow below link for the End User Agreement:

www.umlib.nl/taverne-license

Take down policy

If you believe that this document breaches copyright please contact us at:

repository@maastrichtuniversity.nl

providing details and we will investigate your claim.
}

Copyright and moral rights for the publications made accessible in the public portal are retained by the authors and/or other copyright owners and it is a condition of accessing publications that users recognise and abide by the legal requirements associated with these

- Users may download and print one copy of any publication from the public portal for the purpose of private study or research.

- You may not further distribute the material or use it for any profit-making activity or commercial gain

If the publication is distributed under the terms of Article $25 \mathrm{fa}$ of the Dutch Copyright Act, indicated by the "Taverne" license above, 
Exploration of the sustainability of innovations in hospital care 
The research presented in this thesis was conducted at the School for Public Health and Primary Care: CAPHRI, department Family Practice and at the School for Oncology and Developmental Biology: GROW, of Maastricht University. CAPHRI participates in the Netherlands School of Primary Care Research CaRe.

The studies in this thesis were supported by grants from the Netherlands Organization for Health Research and Development (ZonMw).

(C) Stephanie M.C. Ament, Maastricht 2015

Cover design: Stark Artworks

Layout \& print: Datawyse / Universitaire Pers Maastricht

ISBN: 9789461594549 


\title{
Exploration of the sustainability of innovations in hospital care
}

\author{
PROEFSCHRIFT \\ Ter verkrijging van de graad van doctor aan de Universiteit Maastricht, \\ op gezag van Prof. dr. L.L.G. Soete, Rector Magnificus, \\ volgens het besluit van het College van Decanen, in het openbaar te verdedigen \\ op vrijdag 4 september 2015 om 14:00 uur
}

door

Stephanie Maria Carla Ament

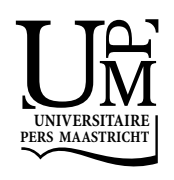




\section{Promotores}

Prof. dr. T. van der Weijden

Prof. dr. C.D. Dirksen

\section{Co-promotor}

Dr. J.M.C. Maessen

\section{Beoordelingscommissie}

Prof. dr. L.W. van Rhijn (voorzitter)

Prof. dr. G.L. Beets

Dr. H. Fiolet

Em. prof. dr. R. Grol, Radboud University Medical Center Nijmegen Prof. dr. H.L. Severens, Erasmus University Rotterdam 


\section{Contents}

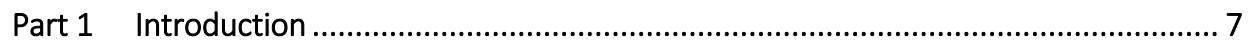

1 General introduction.............................................................................................. 9

2 Sustainability of health care innovations (SUSHI): long-term effects of two implemented surgical care programmes (protocol).................................................... 23

Part 2 Quantitative analyses of the level of sustainability ............................................ 39

3 Sustainability of Short Stay for Breast Cancer Surgery in early adopter hospitals.... 41

4 Sustainability of an Enhanced Recovery After Surgery programme in colonic

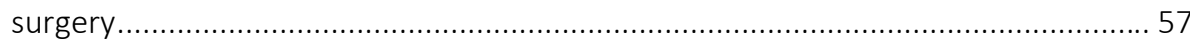

5 Analysing sustainability of economic value using the net monetary benefit approach: The case of a short stay programme for breast cancer surgery care five years after implementation

6 Sustainability of professionals' adherence to guideline recommendations in medical practice: a systematic review.

Part 3 Qualitative analyses of sustainability

7 What determines sustainability of two multidisciplinary hospital-based programmes after achieving early implementation success? A qualitative study... 147

8 Identification of promising strategies to sustain improvements in hospital practice: a qualitative case study.

Part 4 Discussion

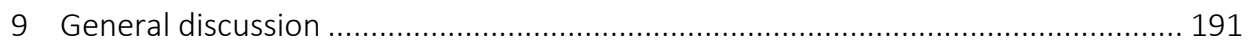

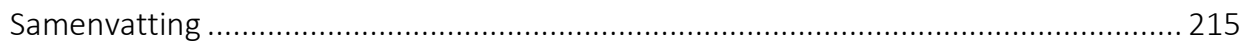

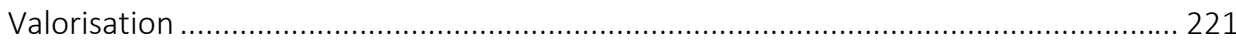

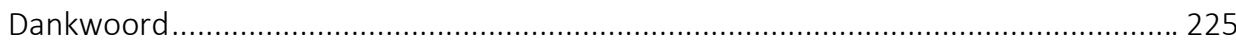

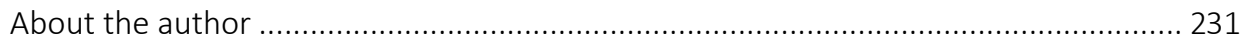





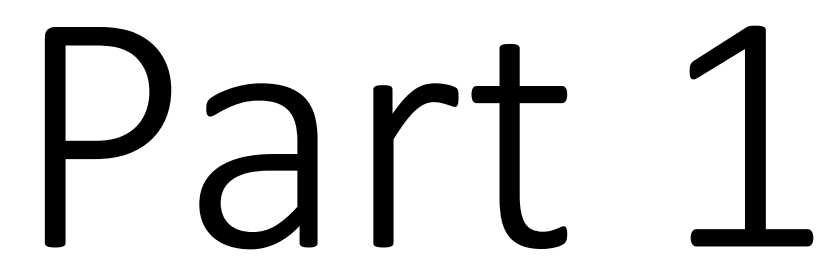

Introduction 



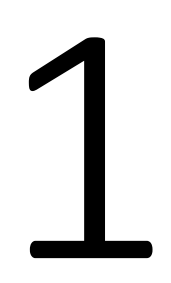

General introduction 
Sustainability has become an upcoming and challenging topic in many fields. Originally, the concept of sustainability is used in environmental and ecological contexts. The Oxford Dictionary states 'sustainable' as 'able to be maintained at a certain rate or level' [1]. In 1987, the Brundtland Commission described 'sustainable development' in an environmental and political context broadly as 'development is sustainable when it meets the needs of the present without compromising the ability of future generations to meet their own needs. It contains within it two concepts: the concept of 'needs', in particular the essential needs of the world's poor, to which overriding priority should be given; and the idea of limitations imposed by the state of technology and social organisation on the environmental's ability to meet present and future needs' [2]. On a macro system level, sustainable development is influenced by societal, environmental and economic determinants which are interrelated and it focuses on creating value with a long-term visionary approach.

In a time when health care is getting more complex and costly, evidence-based innovations need to be implemented to increase the effectiveness and efficiency of care. Unfortunately, many patients still do not receive care following the latest evidence [3]. The harm, of once a patient has accessed health care, majorly contributes to morbidity and mortality $[4,5]$. Besides harm to patients, health care costs may increase as a result of imperfect care delivery [6]. Therefore, an important issue is how quality and efficiency improvements in health care can be achieved.

In health care, innovations are being implemented on a regular basis, leading to various and often unpredictable results [7]. However, if successful implementation of an innovation is achieved, these successes may not be sustained beyond the project timeframe. As Ronald Lippett et al. once described: "Many times an innovation is brought in with great fanfare and is superficially accepted at first. However, months or years later, things have drifted back to the way they were before. Nobody revoked it. It just didn't last" [8]. Many teams that are involved in changing and improving patient care focus on the primary implementation phase, having a short-term lens. It is often unknown to what level the quality improvements have been sustained [9]. Although studies increasingly focus on implementation of evidence, not much research has been performed on the sustainability of health care innovations in practice.

The present thesis explores the concept of sustainability of health care innovations in hospital practice. In this first chapter the content of the thesis is introduced. It starts with background information about innovation and implementation. Innovation and implementation are two components of quality improvement and are applied in practice before the sustainability of change can be analysed. Hereafter, two implementation cases are described, i.e. the short stay programme for breast cancer surgery (SSP) and the enhanced recovery after surgery (ERAS) programme for colonic surgery. These two implementation cases were used for the main research project of the current thesis: the sustainability of health care innovations (SUSHI) study. At the end of this chapter, the aim, the objectives and the outline of this thesis is presented. 


\section{Innovation}

An innovation can be defined as 'a set of ideas, principles and practices that may be adopted in whole or in part' [10]. Moreover, it is a new way of working and it can involve for example changes in a clinical pathway or a new technique. Innovating is an essential focus in every health care setting as it is a way to improve quality or efficiency of care. The complexity of an innovation can be approached using different dimensions, for example by the type of behavior that needs to be changed, by the number of groups or organisational levels which are targeted or by the interacting components of the innovation [11]. Mostly, the recipient of the innovation influences the need for innovation. Rogers described the process of diffusion of innovations to the target group in terms of five categories. Following this theory, the process starts with innovators. Innovators are the first part of a system that take risk by focusing on adoption of an innovation [10]. The second category that is adopting the innovation, are the early adopters followed by the early majority, the late majority and the laggards.

Last decades, a large body of research evidence has become available and from this, new technologies and ideas for process redesigns have emerged. The rate of scientific publications has been increasing over the last 50 years and new insights are increasingly being spread using new distribution channels such as open access journals and publications on the internet [12]. To transfer new available knowledge in health care, clinical practice guidelines are often used. Following the Institute of Medicine (IOM), clinical practice guidelines are "statements that include recommendations intended to optimise patient care that are informed by a systematic review of evidence and an assessment of the benefit and harms of alternative care options" [13]. Clinical practice guidelines contain evidence based advice for professionals and patients and the use of clinical practice guidelines can improve the quality of care [14]. Given the exponential increase in knowledge on innovations, the actual use of the last available evidence has become quite a challenge. There is a growing interest in clinical practice guidelines and they are increasingly being developed and implemented in practice. Key elements of the clinical practice guideline development process are (1) preparing the clinical practice guideline development, (2) systematically reviewing the evidence, (3) drafting the clinical practice guideline and (4) reviewing the clinical practice guideline to evaluate the impact and to possibly update the clinical practice guideline [15-17].

\section{Implementation of innovations}

Rarely, implementation of innovations into practice does happen spontaneously and usually a systematic approach is needed for successful implementation [18]. Implementation strategies can be used to minimise the gap between theoretical and actual implementation effects. Implementation of innovations is complex as the implementation 


\section{CHAPTER 1}

success depends on a variety of factors, such as the innovation, the professional, the patient, professional interactions, incentives and resources, capacity for organisational change; and social, political and legal factors $[19,20]$. Most theoretical models overlap in many dimensions and complement each other. Recently, Damschroder et al. developed the Consolidated Framework Implementation Research (CFIR) which comprises 39 constructs and is based on 19 theories [21]. These constructs are organised into five domains: the characteristics of the innovation, individuals involved, inner setting, outer setting and the implementation process [10, 22-24]. Existing theoretical models often focus on implementation and adoption of innovations rather than on long-term use of innovations in the real-world.

\section{Two implementation cases}

Surgery has a background and a culture to innovate [25]. Traditionally, surgical innovation is described as 'a new or modified surgical procedure that differs from currently accepted local practice, the outcomes of which have not been described, and which may entail risk to the patient' [26]. Like in medical practice in general, innovation is also essential to optimise surgical care delivery. Over the last decades, many innovative advances resulted in substantial quality improvements [27]. New insights emerged and innovations were invented such as minimally invasive surgery and endovascular surgery [28]. This innovative movement in surgery concurs with an increase in cancer patients and an aging population. As a result, surgical care has become more complex and more specialised. Nevertheless, as in other fields, the translation of surgical innovation in practice has been limited due to factors such as inconsistent support by professionals [25].

Between 2005 and 2009, Maastricht University Medical Centre (MUMC) was involved in implementation projects on two evidence-based innovations, the short stay programme for breast cancer surgery (SSP) and the Enhanced Recovery After Surgery (ERAS) programme in colonic surgery. In this thesis, these two surgical implementation cases were used to explore the concept of sustainability. In both implementation cases a clinical practice guideline was implemented to improve the quality of care in a hospital-based and multidisciplinary team setting. Both innovations were externally developed and the implementation strategies were externally guided by expert teams.

\section{Case I: Short Stay Programme for breast cancer surgery}

The global breast cancer incidence is raising with 3.1\% each year [29]. In Europe, the breast cancer incidence rate rises especially in young women [30]. In 2009, 13,000 patients were diagnosed with breast cancer in the Netherlands [31]. The Netherlands has a relatively high incidence level compared to other European countries, which can be explained by high risk factors for breast cancer and the availability of a national screening programme (RIVM). As the majority of breast cancer patients undergo breast sur- 
gery, the increase of the breast cancer incidence will put pressure on hospital and societal costs.

As a result of innovation in surgical care, breast cancer surgery has become less invasive. Also, relatively more patients receive conservative surgery where possible and relatively more patients undergo a sentinel lymph node biopsy instead of complete axillary lymph node dissection in 2010 compared to 2002 [32, 33] Another advance in breast cancer surgery care is treatment in shorter hospital admission. In 2001, the MUMC performed a pilot study in which the feasibility and safety of breast cancer surgery in short stay was tested. Short stay was defined as: 'treating patients in day admission or in overnight admission ( $24 \mathrm{~h}$ admission)' and it could be realised by changing the organisational structure and optimising the patient information. The results of the pilot were promising; the proportion of patients treated in short stay raised from $13 \%$ to $84 \%$ in comparison with traditional breast cancer surgery care in 2001. Four years following the pilot study, a multi-centre implementation study was performed in four early adopter hospitals. The initial implementation aim was to increase the proportion of al types of breast surgery patients treated in short stay by $30 \%$ per centre. First, the project group and the key persons from the participating hospitals defined key recommendations for short stay and identified barriers and facilitators per hospital (table 1). Second, a hospital tailored implementation strategy was executed between 2006 and 2007. A prospectively tailored implementation strategy may be more effective than a generic implementation strategy that is designed without taken barriers into account [34, 35] The hospital tailored strategy consisted of meetings, newsletters, monitoring of results, feedback of performance, reminders and outreach visits. Finally, the implementation project showed an overall increase from $45 \%$ to $82 \%$ of breast cancer surgery treatment in short stay while maintaining quality of care. Treating breast cancer patients in short stay was shown to be effective, safe and efficient. 


\section{CHAPTER 1}

Table 1. Key recommendations to facilitate performing breast cancer surgery care in short stay as implemented in the primary implementation study

Key recommendations

Treatment of breast cancer patient is discussed in a multidisciplinary meeting

The interval between referral and first visit to the breast unit is 5 working days or less

The interval between diagnostic test and informing the patient about the results is 5 working days or less The interval between the decision to operate and surgery is 15 working days or less

The general practitioner is informed about diagnosis, treatment plan and potential side-effects prior to

surgery

The number of preoperative hospital visits is minimised

Patient information about discharge is supported by written information

Specialised home care for patients in the period following surgery has been facilitated

The breast nurse informs the patient about the need for informal care in the home situation

The breast nurse has contacted the patient during the post-operative period

One night of admission is planned for patients with co-morbidity and/or insufficient postoperative supervision Patients planned for day admission are postoperatively given the choice between continuation of admission and discharge

Decisions on patient discharge are based on clear guidelines

\section{Case II: Enhanced Recovery After Surgery for colonic surgery}

In the Netherlands, yearly 13.000 patients undergo colonic surgery as a result of benign and malignant diseases, and the incidence is increasing. Between 1998 and 2001 perioperative care was studied to analyse the gap between evidence based practice and the real-world. This evaluation showed a broad practice variation and revealed care delivery according to traditional surgical routines. Consequently, the ERAS programme in colonic surgery was developed by Fearon et al. The ERAS programme is an evidence-based guideline with perioperative care recommendations (table 2). The programme includes perioperative counselling, no bowel preparation, no preoperative oral fluid restriction, carbohydrate loading up to two hours before surgery, perioperative fluid restrictions, early mobilisation, early oral diet and use of nutritional supplements. Adherence to the guideline has proven to result in faster functional recovery [36-42] and is associated with earlier hospital discharge and research suggested reduced hospital costs [43-45].

As part of the primary implementation study, the ERAS programme was implemented using a breakthrough implementation strategy in 33 hospitals in the Netherlands. The implementation strategy was executed under guidance of the Dutch Institute for Health care Improvement (CBO). The initial implementation aim was to increase the adherence to the ERAS programme elements, to decrease the time needed for functional recovery after surgery and to decrease the length of hospital stay. In every participating hospital, a local implementation project team was established, consisting of a surgeon, an anaesthesiologist, a nurse, a research manager and possibly a hospital manager. The teams attended learning sessions, monitored performance and received feedback in interactive sessions which were organised by the expert team. In the end, 
the implementation study showed that the mean length of hospital stay after colonic surgery dropped from nine to six days and functional recovery decreased from five to three days [46]. Nowadays, the ERAS programme is still considered as best practice in colonic surgery and is being spread to vascular, thoracic, urology and gynaecologic surgery $[47,48]$.

Table 2. Elements of the ERAS protocol as implemented in the primary implementation study

\begin{tabular}{|c|c|}
\hline \multicolumn{2}{|l|}{ Preoperative phase } \\
\hline Preoperative counselling & Extensive information on the ERAS protocol \\
\hline Bowel preparation & None or single phosphate enema \\
\hline Carbohydrate drink & $\begin{array}{l}400 \mathrm{ml} \text { of Pre-op (or comparable) } 2 \text { hours before operation, unless } \\
\text { diabetic }\end{array}$ \\
\hline Sedation & No sedation (short acting if extremely anxious) \\
\hline \multicolumn{2}{|l|}{ Perioperative phase } \\
\hline $\begin{array}{l}\text { Thoracic epidural } \\
\text { analgesia/anaesthesia }\end{array}$ & For all elective bowel surgery \\
\hline Prevention of hypothermia & Temperature $\geq 36.0^{\circ} \mathrm{C}$ at end of operation \\
\hline Thromboembolic prophylaxis & Started $2 \mathrm{~h}$ after epidural and continued until mobilisation \\
\hline Nasogastric tube & Removed before return to ward on day 0 \\
\hline Abdominal drains & No drains \\
\hline Prophylactic antibiotics & 2 drugs, given before incision \\
\hline Incision & Short midline or transverse incisions \\
\hline Oral fluid intake & $800 \mathrm{ml}$ on day 0 (400 $\mathrm{ml}$ if operated in afternoon) \\
\hline Sitting up & At least 15 minutes on day 0 \\
\hline \multicolumn{2}{|l|}{ Postoperative phase } \\
\hline IV fluids & Stopped on day 1 \\
\hline Normal diet & Resumed on day 1 \\
\hline Oral supplements & $200 \mathrm{ml}$ twice daily from day 1 \\
\hline Sitting up & At least 3 times 30 minutes in chair on day 1 \\
\hline Oral laxatives & MgO tablets 1000 mg from day 1 \\
\hline Oral analgesics & $\begin{array}{l}\text { Paracetamol from day 0; NSAID started before removal of epidural } \\
\text { catheter }\end{array}$ \\
\hline Removal of epidural catheter & On day 2 \\
\hline Removal of urinary catheter & At the time of removal of the epidural catheter \\
\hline
\end{tabular}

\section{Sustainability}

In the field of health care a sustainability approach is urgently needed to keep up with the growing health care demand and the limited available resources. In 2008, the Institute for Health care Improvement (U.S.) introduced the 'triple aim' policy approach: '(1) improving the individual experience of care; (2) improving the health of populations; 
and (3) reducing the per capita costs of care for populations' [49]. The triple aim is a statement aiming to improve health care systems while reducing its costs. This approach should increase the chance of creating a sustainable health care system.

In this thesis, sustainability in the context of implementation science is explored. Sustainability of health care innovations after quality improvement efforts is a relatively new concept. Different definitions for sustainability are used in the literature and there are different views about what needs to be sustained [50]. Sustainability can be studied from different perspectives [51]. For example, different settings are studied and different methods and definitions are used. Some definitions focus on maintaining benefits (outcomes) while some other definitions focus on institutionalisation of a programme in the organisation (structure and process). Often, sustainability is described as "maintaining the health benefits", "continuation of the programme activities within an organisational structure " and "building the capacity of a recipient community" [52, 53]. The phenomenon of not maintaining achieved implementation results is called 'evaporation' by the National Health Service (NHS) [54] and 'decay' by Buchanan et al. [55].

Sustainability of health care innovations is characterised as a dynamic process [56, 57]. Scheirer et al. [52] conducted a systematic review of sustainability research in the Canadian and U.S. health care setting. They presented five determinants of sustainability of a new health care programme: the programme can be modified over time, a champion is present, the programme fits with its organisation mission and procedures, benefits to staff or patients are perceived, and stakeholders in other organisations support the innovation. The factors fit in the framework for conceptualising programme sustainability in health education and health promotion as proposed by ShediacRizkallah and Bone [53]. Their framework describes determinants in three main categories of determinants: project design and implementation factors, factors within the organisational setting and factors in the broader community environment. The conclusion of both studies was that the determinants of sustainability varied and that sustainability was studied in different health care fields using different methods. Furthermore, in another systematic review about sustainability in public health, Gruen et al. concluded that the concept of sustainability is underdeveloped as a result of limited research [50]. Changing health care practice is difficult to achieve and not much research has been performed concerning sustainability of health care innovations. Also, there is a lack of guidance by a conceptual framework and a methodology to determine the level of sustainability.

This thesis focuses on the sustainability of health care innovations in medical care, particularly clinical practice guidelines for hospital care. The thesis follows up on longterm quality improvement research of two implementation projects. The sustainability of two multidisciplinary hospital-based innovations was studied from a multidimensional perspective. These two innovations were complex innovations and achieving practice change was challenging, involving many departments and multidisciplinary team members. Primary implementation research focused on the safety, effectiveness, efficiency 
and implementation of the innovations and the early post-implementation results showed successful transformation of care in both cases. However, investments and efforts to change practice are a waste of money and time if achieved quality improvement results decrease unintended in the post-implementation phase. Unfortunately, evidence about whether the implementation successes sustained beyond the cessation of the projects was still missing.

\section{Objective}

The overall objective of this thesis was to improve long-term impact of implementation efforts by exploring the concept of sustainability of innovations in hospital care. This thesis assessed and evaluated the sustainability of SSP and the ERAS programme three to six years following implementation. Sustainability of these two innovations was analysed by means of various evaluations. This research is unique in the field of implementation science while it focuses on the concept of sustainability in hospital care. The research questions are:

- To what level were health care innovations sustained in the late post-implementation phase?

- To what level was the economic value of the short stay programme for breast cancer surgery sustained in four early adopter hospitals?

- What were the determinants of the sustainability of the two innovations three to six years after implementation, as perceived by the professionals?

- What strategies seem to be promising for sustaining the ERAS programme for colonic surgery, as perceived by the professionals?

\section{Outline of the thesis}

This thesis consists of four parts and nine chapters. The first part (chapters 1-2) provides an introduction to the thesis. The protocol of the Sustainability of Health care Innovations (SUSHI) study is presented in chapter 2.

In the second part (chapters 3-6), multiple sustainability evaluations are presented to determine to what level innovations are sustained. In this part, sustainability is evaluated in terms of key outcomes at patient level, professionals' adherence to the innovative guideline, and the economic value of sustaining the innovation. The level of sustainability in terms of outcomes and adherence of SSP and the ERAS programme is presented in respectively chapter 3 and 4 . Chapter 5 introduces the concept of sustainability of economic value. In this chapter sustainability of economic value of SSP is analysed using the net monetary benefit approach. The second part ends with chapter 6, a sys- 


\section{CHAPTER 1}

tematic review of the sustainability of professionals' adherence to clinical practice guidelines in medical care.

Part three (chapters 7,8 ) explores the concept of sustainability using a qualitative method. In chapter 7 the key determinants of the sustainability in terms of early implementation successes of the two hospital-based multidisciplinary innovations were studied. Perceived key determinants that explain sustainability were analysed three to six years following successful implementation. In chapter 8 , strategies that support, have supported or have the potential to support the sustainability of the ERAS programme after joining a quality improvement collaborative are presented. Part four is the final part of the thesis (chapter 9). Chapter 9 provides an overall conclusion and the general discussion of this thesis. As a result of the fact that most papers have been published, overlap in information may occur. 


\section{References}

1. Oxford Dictionaries. "sustainable". Oxford University Press. [cited 2014 Nov 16] Available from: http://www.oxforddictionaries.com/definition/english/sustainable

2. World Commission on Enverinment and Development. Our Common future. Oxford: Oxford University Press, 1987.

3. European Science Foundation. Implementation of Medical Research in Clinical Practice: Forward Look. European Science Foundation. 2011.

4. Jha AK, Larizgoitia I, Audera-Lopez C, Prasopa-Plaizier N, Waters H, Bates DW. The global burden of unsafe medical care: analytic modelling of observational studies. BMJ Quality \& Safety. 2013 October 1, 2013;22(10):809-15.

5. James JT. A new, evidence-based estimate of patient harms associated with hospital care. Journal of patient safety. 2013 Sep;9(3):122-8

6. Newman-Toker DE, Pronovost PJ. Diagnostic errors--the next frontier for patient safety. JAMA. 2009 Mar 11;301(10):1060-2.

7. Guldbrandsson K, folkhälsan Sif. From News to Everyday Use: The Difficult Art of Implementation : a Literature Review: Swedish National Institute of Public Health; 2008.

8. Senft P. THE DYNAMICS OF PLANNED CHANGE. By Ronald Lippitt, Jeanne Watson and Bruce Westley, under the Editorship of Willard B. Spalding. New York: Harcourt, Brace \& Company, 1958. Pp. viii + 312. International Journal of Social Psychiatry. 1960 April 1, 1960;5(4):315-6.

9. Øvretveit J, Bate P, Cleary P, Cretin S, Gustafson D, Mclnnes K, et al. Quality collaboratives: lessons from research. Quality and Safety in Health Care. 2002 December 1, 2002;11(4):345-51.

10. Rogers E. Diffusion on innovations. 5th edition ed. New York: NY: Free Press; 2003.

11. Council MR. Developing and evaluating complex interventions: new guidance. 2008.

12. Larsen PO, von Ins $\mathrm{M}$. The rate of growth in scientific publication and the decline in coverage provided by Science Citation Index. Scientometrics. 2010 Sep;84(3):575-603.

13. Institute of Medicine. Graham R MM, Wolman DM, Greenfield S, Steinberg E, editor(s). Clinical practice guidelines we can trust. . Washington (DC): 2011

14. Thomas LH, McColl E, Cullum N, Rousseau N, Soutter J, Steen N. Effect of clinical guidelines in nursing, midwifery, and the therapies: a systematic review of evaluations. Quality in Health Care. 1998 December 1, 1998;7(4):183-91.

15. Turner T, Misso M, Harris C, Green S. Development of evidence-based clinical practice guidelines (CPGs): comparing approaches. Implementation Science. 2008;3(1):45.

16. Tuut K, Pinkhof H. Pinkhof Zakwoordenboek 'evidence-based' geneeskunde: Bohn Stafleu van Loghum; 2011

17. Regieraad Kwaliteit van Zorg. Richtlijn voor richtlijnen. Den Haag: 2012.

18. van Bokhoven MA, Kok G, van der Weijden T. Designing a quality improvement intervention: a systematic approach. Quality \& safety in health care. 2003 Jun;12(3):215-20.

19. Flottorp SA, Oxman AD, Krause J, Musila NR, Wensing M, Godycki-Cwirko M, et al. A checklist for identifying determinants of practice: a systematic review and synthesis of frameworks and taxonomies of factors that prevent or enable improvements in healthcare professional practice. Implement Sci. 2013;8:35.

20. R. Grol MW, M. Eccles, D. Davis. Improving patient care: the implementation of change in health care. John Wiley \& Sons; 2013.

21. Damschroder LJ, Aron DC, Keith RE, Kirsh SR, Alexander JA, Lowery JC. Fostering implementation of health services research findings into practice: a consolidated framework for advancing implementation science. Implement Sci. 2009;4:50.

22. Damschroder LJ, Hagedorn HJ. A guiding framework and approach for implementation research in substance use disorders treatment. Psychol Addict Behav. 2011 Mar 28.

23. Simpson DD, Flynn PM. Moving innovations into treatment: A stage-based approach to program change. $J$ Subst Abuse Treat. 2007 Sep;33(2):111-20 


\section{CHAPTER 1}

24. Greenhalgh T, Robert G, Macfarlane F, Bate P, Kyriakidou O. Diffusion of innovations in service organizations: systematic review and recommendations. The Milbank quarterly. 2004;82(4):581-629.

25. Riskin DJ, Longaker MT, Gertner M, Krummel TM. Innovation in surgery: a historical perspective. Annals of surgery. 2006 Nov;244(5):686-93.

26. Biffl WL, Spain DA, Reitsma AM, Minter RM, Upperman J, Wilson M, et al. Responsible development and application of surgical innovations: a position statement of the Society of University Surgeons. Journal of the American College of Surgeons. 2008 Jun;206(3):1204-9.

27. Barkun JS, Aronson JK, Feldman LS, Maddern GJ, Strasberg SM, Altman DG, et al. Evaluation and stages of surgical innovations. Lancet. 2009 Sep 26;374(9695):1089-96.

28. Mathis KL, Cima RR, Pemberton JH. New developments in colorectal surgery. Current opinion in gastroenterology. 2011 Jan;27(1):48-53.

29. Forouzanfar MH, Foreman KJ, Delossantos AM, Lozano R, Lopez AD, Murray CJ, et al. Breast and cervical cancer in 187 countries between 1980 and 2010: a systematic analysis. Lancet. 2011 Oct 22;378(9801): 1461-84.

30. Leclere B, Molinie F, Tretarre B, Stracci F, Daubisse-Marliac L, Colonna M. Trends in incidence of breast cancer among women under 40 in seven European countries: a GRELL cooperative study. Cancer epidemiology. 2013 Oct; 37(5):544-9. .

31. Gommer AM (RIVM) PMR, Voogd AC (UM). Prevalentie, incidentie en sterfte naar leeftijd en geslacht. Kanker,Borstkanker Bilthoven: RIVM; 2010 [cited 2013 Oct 28]. Available from: http://www.nationaalkompas.nl.

32. Driul L, Bernardi S, Bertozzi S, Schiavon M, Londero AP, Petri R. New surgical trends in breast cancer treatment: conservative interventions and oncoplastic breast surgery. Minerva ginecologica. 2013 Jun;65(3):289-96. PubMed PMID: 23689171. Epub 2013/05/22. eng.

33. Mascaro A, Farina M, Gigli R, Vitelli CE, Fortunato L. Recent advances in the surgical care of breast cancer patients. World journal of surgical oncology. 2010;8:5.

34. Baker R, Camosso-Stefinovic J, Gillies C, Shaw EJ, Cheater F, Flottorp S, et al. Tailored interventions to overcome identified barriers to change: effects on professional practice and health care outcomes. Cochrane Database Syst Rev. 2010 (3):CD005470.

35. Sinnema $H$, Terluin B, Wensing M, Volker D, Franx G, van Balkom A, et al. Systematic tailoring for the implementation of guideline recommendations for anxiety and depressive disorders in general practice: perceived usefulness of tailored interventions. BMC family practice. 2013;14:94.

36. Gustafsson UO, Scott MJ, Schwenk W, Demartines N, Roulin D, Francis N, et al. Guidelines for perioperative care in elective colonic surgery: Enhanced Recovery After Surgery (ERAS(R)) Society recommendations. Clin Nutr. 2012 Dec;31(6):783-800

37. Kehlet $\mathrm{H}$, Mogensen $\mathrm{T}$. Hospital stay of 2 days after open sigmoidectomy with a multimodal rehabilitation programme. Br J Surg. 1999 Feb;86(2):227-30.

38. Basse L, Hjort Jakobsen D, Billesbolle P, Werner M, Kehlet $\mathrm{H}$. A clinical pathway to accelerate recovery after colonic resection. Annals of surgery. $2000 \mathrm{Jul} ; 232(1): 51-7$.

39. Basse L, Raskov HH, Hjort Jakobsen D, Sonne E, Billesbolle P, Hendel HW, et al. Accelerated postoperative recovery programme after colonic resection improves physical performance, pulmonary function and body composition. Br J Surg. 2002;89(4):446-53.

40. Basse L, Thorbol JE, LossI K, Kehlet H. Colonic surgery with accelerated rehabilitation or conventional care. Dis Colon Rectum. 2004 Mar;47(3):271-7; discussion 7-8.

41. Kehlet H, Wilmore DW. Multimodal strategies to improve surgical outcome. Am J Surg. 2002 Jun;183(6) 630-41.

42. Varadhan KK, Neal KR, Dejong $\mathrm{CH}$, Fearon KC, Ljungqvist O, Lobo DN. The enhanced recovery after surgery (ERAS) pathway for patients undergoing major elective open colorectal surgery: a meta-analysis of randomized controlled trials. Clin Nutr. 2010 Aug;29(4):434-40.

43. Adamina M, Kehlet $H$, Tomlinson GA, Senagore AJ, Delaney CP. Enhanced recovery pathways optimize health outcomes and resource utilization: a meta-analysis of randomized controlled trials in colorectal surgery. Surgery. 2011 Jun;149(6):830-40. 
44. Sammour T, Zargar-Shoshtari K, Bhat A, Kahokehr A, Hill AG. A programme of Enhanced Recovery After Surgery (ERAS) is a cost-effective intervention in elective colonic surgery. The New Zealand medical journal. 2010 Jul 30;123(1319):61-70.

45. Maessen J, Dejong CH, Hausel J, Nygren J, Lassen K, Andersen J, et al. A protocol is not enough to implement an enhanced recovery programme for colorectal resection. Br J Surg. 2007 Feb;94(2):224-31.

46. Gillissen F, Hoff C, Maessen JMC, Winkens B, Teeuwen JH, von Meyenfeldt MF, et al. Structured synchronous implementation of an enhanced recovery program in elective colonic surgery in 33 hospitals in The Netherlands. World journal of surgery. 2013 May;37(5):1082-93.

47. Coolsen MM, Wong-Lun-Hing EM, van Dam RM, van der Wilt AA, Slim K, Lassen K, et al. A systematic review of outcomes in patients undergoing liver surgery in an enhanced recovery after surgery pathways. HPB : the official journal of the International Hepato Pancreato Biliary Association. 2013 Apr;15(4):245-51.

48. Lv D, Wang X, Shi G. Perioperative enhanced recovery programmes for gynaecological cancer patients. The Cochrane database of systematic reviews. 2010 (6):CD008239.

49. Berwick DM, Nolan TW, Whittington J. The Triple Aim: Care, Health, And Cost. Health Affairs. 2008 May 1 , 2008;27(3):759-69.

50. Hanson HM, Salmoni AW, Volpe R. Defining program sustainability: differing views of stakeholders. Canadian journal of public health = Revue canadienne de sante publique. 2009 Jul-Aug;100(4):304-9.

51. Gruen RL, Elliott JH, Nolan ML, Lawton PD, Parkhill A, McLaren CJ, et al. Sustainability science: an integrated approach for health-programme planning. Lancet. 2008 Nov 1;372(9649):1579-89.

52. Scheirer M. Is sustainability possible? A review and commentary on empirical studies of program sustainability Am J Eval. 2005;26:320-47.

53. Shediac-Rizkallah MC, Bone LR. Planning for the sustainability of community-based health programs: conceptual frameworks and future directions for research, practice and policy. Health Educ Res. 1998 Mar;13(1):87-108.

54. NHS. Sustainability and its relationship with spread and adoption, General improvement skills. Coventry, UK: Improvement Leaders' guide; 2007

55. Buchanan D, Fitzgerald L, Ketley D, Gollop R, Jones JL, Lamont SS, et al. No going back: A review of the literature on sustaining organizational change. International Journal of Management Reviews. 2005;7(3): 189-205.

56. Johnson K, Hays C, Center H, Daley C. Building capacity and sustainable prevention innovations: a sustainability planning model. Evaluation and Program Planning. 2004 5//;27(2):135-49.

57. Pluye P, Potvin L, Denis JL, Pelletier J. Program sustainability: focus on organizational routines. Health Promot Int. 2004 Dec;19(4):489-500 



\section{Sustainability of health care innovations}

(SUSHI): long-term effects of two implemented surgical care programmes (protocol)

Stephanie M C Ament*, Freek Gillissen*, José M C Maessen, Carmen D Dirksen, Trudy van der Weijden, Maarten F von Meyenfeldt

Published: BMC Health Services Research 2012, 12:423

* both authors contributed equally 


\section{CHAPTER 2}

\section{Abstract}

\section{Background}

Two health care innovations were successfully implemented using different implementation strategies. First, a short stay programme for breast cancer surgery (SSP) was implemented in four early adopter hospitals, using a hospital-tailored implementation strategy. Second, the Enhanced Recovery After Surgery (ERAS) programme for colonic surgery was implemented in 33 Dutch hospitals, using a generic breakthrough implementation strategy. Both strategies resulted in a shorter hospital length of stay without a decrease in quality of care. Currently, it is unclear to what level these innovative programmes and their results have been sustained three to six years following implementation. The aim of the sustainability of health care innovations (SUSHI) study is to analyse sustainability and its determinants using two implementation cases.

\section{Methods}

This observational study uses a mixed methods approach. The study will be performed in 14 hospitals in the Netherlands, from November 2010. For both implementation cases, the programme aspects and the effects will be evaluated by means of a follow-up measurement in 160 patients who underwent breast cancer surgery and 300 patients who underwent colonic surgery. A policy cost-effectiveness analysis from a societal perspective will be performed prospectively for the short stay programme for breast cancer surgery in 160 patients. To study determinants of sustainability key professionals in the multidisciplinary care processes and implementation change agents will be interviewed using semi-structured interviews.

\section{Discussion}

The concept of sustainability is not commonly studied in implementation science. The SUSHI study will provide insight in to what level the short-term implementation benefits have been maintained and in the determinants of long-term continuation of programme activities. 


\section{Background}

Quality improvement strategies are used to change and improve health care. However, often health care innovations barely get incorporated in every day practice [1-4]. Implementing an innovation is a complex process. Studies show that $30-40 \%$ of patients do not receive health care according to evidence-based knowledge and $20-25 \%$ of the health care provided is not needed or potentially harmful [5-7]. There is a high level of agreement between all parties involved in health care that the quality of care could be improved. However, there is still no consensus on how to achieve better care. A diversity of quality improvement strategies to change and improve health care routines has been proposed. However, there is not one strategy that is superior in its effect above other strategies in improving health care processes [7]. Although magic bullets are lacking among the prevailing implementation strategies, evidence suggests that implementation strategies which are systematically tailored to the actual and perceived barriers and facilitators for change increase the success of implementation $[8,9]$.

Less is known on the long-term effectiveness of implementation strategies. Even after successful implementation, practice seems to indicate that there is a tendency to return to old routines after implementation activities have ended. It can be argued that without consolidation of the achieved benefits, implementation strategies are a waste of time and money. Implementation strategies' 'good value for money' is determined by its ability to implement evidence based clinical guidelines into daily routines and by the implementation costs [10]. The implication of discontinued programmes is however not just financial but can also result in less optimal care and service for patients. Moreover, it can also diminish community trust and support for future programmes. Therefore, it is important that achieved benefits of a proven effective intervention are sustained after implementation.

Sustainability is a relatively new term in health care research, but has become an issue of growing interest. There is no uniform definition of sustainability in the literature [11]. In its simplest form, it can be seen as 'holding the gains' and 'evolving as required'. Different definitions regarding sustainability are used, for example "maintaining the health benefits [12]", "continuation of the programme activities within an organisational structure [13]" and "building the capacity of a recipient community [14]" [11, 15, 16]. Sustainability is generally seen as a dynamic process of continuous improvement. In the current study we use the following definition: "Sustainability of change exists when a newly implemented innovation continues to deliver the achieved benefits over a longer period of time, certainly does not return to the usual processes and becomes 'the way things are done around here', until a better innovation comes along, even after the implementation project is no longer actively carried out $[11,17]$ "

Sustainability is obtaining its position in health care research, but existing work on sustainability has mainly been undertaken from a pragmatic perspective and was impoverished by a lack of process information [18]. Several determinants can have a facili- 


\section{CHAPTER 2}

tating or impeding effect on sustainability. Important questions that still need to be answered are by what strategies particular health care innovations are implemented and sustained (or not) in particular contexts and settings, and whether these strategies can be improved.

Few empirical studies have specifically addressed the implementation and sustainability of innovations. In the current study two Dutch implementation cases are used for research on sustainability and its determinants. A short stay programme (SSP) for breast cancer surgery was implemented in four early adopter hospitals by means of a hospital tailored implementation strategy [19-22], and the Enhanced Recovery After Surgery (ERAS) programme in colonic surgery was implemented in 33 hospitals by means of a generic implementation strategy $[23,24]$. As a result of these efforts (table 1), both cases showed improved short-term results in terms of hospital length of stay. However, it is currently unknown to what level and how these programmes and their results have been sustained.

Table 1. Overview innovation cases

\begin{tabular}{|c|c|c|}
\hline & SSP & ERAS \\
\hline No of participating hospitals & 4 & 33 \\
\hline Innovation & $\begin{array}{l}\text { Ultra short stay programme after breast } \\
\text { cancer surgery }\end{array}$ & $\begin{array}{l}\text { ERAS programme in colonic } \\
\text { surgery }\end{array}$ \\
\hline Implementation strategy & Hospital tailored strategy & The Breakthrough Series \\
\hline Implementation time span & $2005-2007$ & $2006-2009$ \\
\hline Measurement periods & $\begin{array}{l}\text { Two measurement periods of six months, } \\
\text { with six months of actual implementation } \\
\text { in between }\end{array}$ & Three runs of each one year \\
\hline
\end{tabular}

\section{Objectives}

In the present study we will assess the sustainability of a short stay programme for breast cancer surgery and the Enhanced Recovery After Surgery programme in colonic surgery, three to six years after their implementation.

The research questions are:

1. To what level have the achieved benefits of the short stay programme for breast cancer surgery and the Enhanced Recovery After Surgery programme in colonic surgery been sustained?

2. What is the current policy cost-effectiveness of the short stay programme for breast cancer surgery?

3. Which are the determinants of sustainability as perceived by the professionals of the short stay programme for breast cancer surgery and the Enhanced Recovery After Surgery programme in colonic surgery? 


\section{Methods}

\section{Design}

The present study is an observational study using a mixed methods approach, applied to the two implementation cases. First, by means of a retrospective analysis of patient files we will analyse process and outcomes of care to assess the level of sustainability. Second, the policy cost-effectiveness will be calculated for the short stay programme based on prospective data from breast cancer surgery patients. The cost-effectiveness analysis will use the same methodology as in the primary implementation study [19]. Third, the determinants of sustainability will be explored by means of in-depth semi-structured interviews with relevant stakeholders and external change agents. This study will be executed between November 2010 and November 2013

\section{Ethical approval and informed consent}

The Medical Ethical Committee of the University of Maastricht has granted approval, METC 11-4-015.10. The privacy of the included patients is protected, and all data will be coded and processed anonymously. Medical files with explicit patient statements that their medical information should not be used for clinical research will not be included Breast cancer patients eligible for the second research question in this study will be asked for informed consent for prospective collection of quality of life and cost data.

\section{Participants}

\section{Hospitals}

Hospitals participating in the breast cancer surgery case are those four hospitals which participated in the primary implementation strategy, covering four main organisational hospital settings in the Netherlands. The uptake of the short stay programme for breast cancer surgery was successful in all four hospitals; after implementation the average proportion of patients treated in short stay was increased with $36 \%$.

Hospitals participating in the colonic surgery case were selected out of the 33 hospitals which participated in the primary implementation strategy. Selection was based on the criterion that the primary implementation strategy was successfully executed in these hospitals, such that the chance of finding information on sustainability and its determinants is increased. Success was defined as follows: (1) a median hospital length of stay of six days or lower, (2) an overall protocol compliance above seventy percent, and (3) at least forty patients treated according to the ERAS programme. Nine hospitals fulfilled these criteria. One hospital was selected on top of that, based on being a successful early adopter hospital. Ten hospitals were selected for the SUSHI study. 


\section{CHAPTER 2}

Research question one: To what level have the achieved benefits of the short stay programme for breast cancer surgery and the Enhanced Recovery After Surgery programme in colonic surgery been sustained?

\section{Participants}

\section{Patients}

Data collection will be conducted among the last 160 consecutive patients who had been scheduled for breast cancer surgery in short stay ( 40 per centre) and the last 300 consecutive patients who underwent colonic surgery (30 per centre), who met the same inclusion criteria as in the primary implementation studies. Patients in the breast cancer surgery case are all patients aged over 18 years, diagnosed with breast cancer, and who underwent surgery. Patients whose physiology at diagnosis impedes participation in the short stay programme as assessed by the breast surgeon, anaesthesiologist and nurse must be scheduled for at least one overnight stay. Also, patients who cannot rely on sufficient informal home care during the first night after surgery and patients with complaints that necessitate postoperative monitoring must be scheduled for at least one overnight stay. Patients included in the colonic surgery case are all patients aged over 18 undergoing an elective colonic resection above the peritoneal reflection for both malignant and benign diseases. Patients undergoing emergency surgery and requiring an ileo- or colostomy are excluded.

\section{Variables and measurements}

Outcome and process of care will be measured by means of pre-defined indicators to determine the level of sustainability of both programmes, which will be extracted from patient files. The primary outcome measure in the breast cancer surgery case is the proportion of breast cancer surgery patients treated in day care admission or one overnight stay. Secondary outcome measure is the number of complications. The primary outcome measure in the colonic surgery case is the hospital length of stay (LOS). Secondary outcome measure is functional recovery (FR), reached when a patient is tolerating solid foods, is comfortable on oral analgesics only, and mobilised at preoperative level [25]. Baseline patient characteristics and possible reasons for delay in discharge (the gap between FR and LOS) will be recorded (table 2). The process indicators are shown in table 3. For both the breast cancer surgery and colonic surgery case, the outcome measure is the adherence to the protocol. Adherence to the protocol will be determined per process indicator and per patient. Reasons for non-adherence to specific guideline elements will be recorded if available. 
SUSTAINABILITY OF HEALTH CARE INNOVATIONS (PROTOCOL)

Table 2. Outcome indicators and baseline characteristics scored in both cases

\begin{tabular}{ll}
\hline SSP & ERAS \\
\hline Primary outcome & Primary outcome \\
Treated surgically in day care & Postoperative length of hospital stay \\
Treated surgically in overnight stay & Day functional recovery was reached \\
Baseline characteristics & Baseline characteristics \\
Patient characteristics & Patients characteristics \\
Eligible for surgery in short stay & Laparoscopic or open approach \\
Receiving breast conserving surgery & Different types of operations \\
\hline
\end{tabular}

Table 3. Process indicators in both cases

\begin{tabular}{ll}
\hline SSP & ERAS \\
\hline Preoperative counselling & Preoperative counselling \\
Not planned for short stay admission despite & No preoperative bowel preparation \\
fulfilling the inclusion criteria for short stay & Preoperative PreOp carbohydrate drink \\
admission & Epidural anaesthesia/analgesia \\
Being offered home care nursing after breast & Perioperative warming (Bair hugger) \\
cancer surgery & No abdominal drains placed during surgery \\
The reasons for discrepancy between fulfilling the & Nasogastric tube removed after surgery \\
inclusion criteria for short stay admission and & Nutritional supplements postoperatively \\
being scheduled for inpatient admission & Mobilisation $>15$ minutes at day 0 \\
The reasons for not being treated in short stay & Use of oral fluids $>500$ ml at day 0 \\
despite being scheduled for short stay & Mobilisation $>3$ hours at day 1 \\
& Intravenous fluid infusion stopped at day \\
& Resumption of solid food at day 1 \\
& Removal of epidural analgesia on day 2 \\
& Oral laxatives postoperatively \\
& The reasons for not adhering to these different protocol \\
& elements
\end{tabular}

\section{Data analysis}

Descriptive statistics (proportions, mean, median) will be performed for both outcome data sets. The percentage adherence to the protocol will be calculated per programme element for both cases and an overall protocol adherence score will be calculated per patient. All statistical analyses will be performed using SPSS 18.0 (SPSS Inc. Chicago, Illinois, USA).

Research question two: What is the current policy cost-effectiveness of the short stay Programme for breast cancer surgery? 


\section{CHAPTER 2}

\section{Participants}

In this prospective part of the study 40 consecutive patients operated per centre ( $n=160$ $(4 \times 40))$ will be included. Again we will use the same inclusion and exclusion criteria as in the primary implementation study.

\section{Variables and measurements}

\section{Policy cost analysis}

The cost analysis will be performed from a societal perspective, using the Dutch guidelines for cost calculations in health care [26]. Policy costs represent both the programme and implementation costs [27]. The short stay programme for breast cancer surgery related direct and indirect health care and non-health care costs will be measured and valued. We will use a bottom up micro costing method which identifies and measures the health care products per patient [26]. Costs will be expressed in 2012 Euros. Direct health care related resource use will be obtained using Case Record Forms. Direct costs outside health care and out of pockets costs will be collected through a cost diary filled out by patients. One day before surgery, patients will fill out the retrospective part of the cost book to determine the health care related costs for a period of two weeks before surgery. Patients will fill out the prospective part of the cost book during six weeks from the moment of discharge. This time horizon will cover most costs related to surgical treatment and was also used in the primary implementation study. To perform the cost calculations, the volumes of (health care) resource use are multiplied with the cost prices per unit of resource use. Cost prices from the primary study will be actualised and used in this study. Indirect costs are the productivity losses due to sick leave; these will be calculated using the friction costs method. A friction cost method confines productivity losses to the period needed to replace a sick worker. Historical implementation costs of the short stay programme for breast cancer surgery will be used in this study, as these costs have been made in the primary implementation study. The implementation costs were assessed as mean costs per patient.

\section{Effectiveness}

Effectiveness will be determined through a generic health-related quality of life (HRQoL) instrument. In this study the EuroQol (EQ-5D) will be used to calculate quality-adjusted life years (QALYS) [28]. The EQ-5D consists of five different dimensions (mobility, selfcare, daily activities, pain/discomfort, depression/anxiety). Every dimension has three answer possibilities (no problems, some problems, severe problems), which can lead to 243 different health states. The health states as measured in this study will be used for utility score calculation, based on the UK tariff. The utility scores will be determined at four consecutive time points: one day before first surgery (at baseline), one day after first surgery, one week after first surgery and six weeks after first surgery. 


\section{Data analyses}

In this study the current incremental cost-effectiveness ratio (ICER) will be calculated for the short stay programme for breast cancer surgery versus care as usual before primary implementation. For calculation of the ICER, mean incremental policy costs will be divided by the mean incremental QALYs. Multiple imputations will be used to replace missing values with plausible estimates. Bootstrapping will be performed to determine 95\% confidence intervals around cost differences between the short stay programme for breast cancer surgery and care as usual. Bootstrapping will also be used to quantify the uncertainty around the ICER and will be performed using Excel 2003 [29]. The results will be presented in an incremental cost-effectiveness plane in which the vertical axis will represent the incremental effects and the horizontal axis will show the incremental costs between the short stay programme and care as usual before implementation. This will result in four quadrants: 1) southeast quadrant (SE) 2) northwest quadrant (NW) 3) southwest quadrant (SW) 4) northeast quadrant (NE). The costeffectiveness acceptability curve will be calculated to present the probability of the short stay programme being cost-effective for a range of ceiling ratios using Excel 2003. These analyses will be performed using SPSS 18.0 (SPSS Inc. Chicago, Illinois, USA). Sensitivity analyses will be performed to test the robustness of the results for changing several parameters, as well as subgroup analyses. In addition, current policy costs and effects of the short stay programme will be compared to policy costs and effects as calculated in the primary implementation study, and potential differences will be related to sustainability issues when applicable.

Research question three: Which are the determinants of sustainability as perceived by the professionals of the short stay programme for breast cancer surgery and the Enhanced Recovery After Surgery programme in colonic surgery?

\section{Respondents}

\section{Professionals}

Professionals within the participating hospitals will be interviewed. The first interview will be conducted with the responsible surgeon. Following this interview approximately 1-2 key persons involved in the actual care process will be selected per hospital. The number of interviews may differ on hospital level based on the qualitative research process. Before the start of the interview, the interviewees will be formally informed about the relevance, expected duration, confidentiality of the personal data and the permission to audio tape the interviews. 


\section{CHAPTER 2}

\section{External change agents}

External change agents involved in the primary implementation strategies will also be invited for an interview to explore their perspective on sustainability in relation to the implementation processes and results.

\section{Variables and measurements}

The interview schedule (additional file 1) for the semi structured interviews was developed based on a list of relevant topics inspired by the Consolidated Framework Implementation Research (CFIR) model, recently developed by Damschroder et al [30]. The CFIR model (figure 1 ) is composed of 39 factors. These factors are organised into five constructs: the characteristics of the innovation, individuals involved, inner setting, outer setting and the implementation process [31-34]. Furthermore, the interview will be guided by information regarding current process and outcomes of care in comparison to historical results following primary implementation. The interviews will be held by one of the researchers (either SA or FG), who were not involved in the primary implementation process. First, after a short introduction the interviewees' perception of sustainability in their hospital will be questioned. Second, hospital-specific information regarding current practice in comparison to historical results following primary implementation will be graphically displayed, and interviewees will be asked to reflect on these results. Third, interviewees will be questioned about the innovation itself, hospital culture, incentives and other factors possibly influencing sustainability. The interviews will be audio taped for documentation and analysis to guarantee transparency. Interviews will take about forty-five minutes. Immediately afterwards the researcher will make field notes. Summaries of the main findings per transcript will be sent to the particular hospitals to perform a member check.

\section{Data analysis}

The interviews will be transcribed and independently coded by both researchers (SA and FG). Differences in coding will be discussed until consensus is reached. Research software NVivo will be used to analyse the data and progress in coding and analysis will be discussed in the research team. 
SUSTAINABILITY OF HEALTH CARE INNOVATIONS (PROTOCOL)

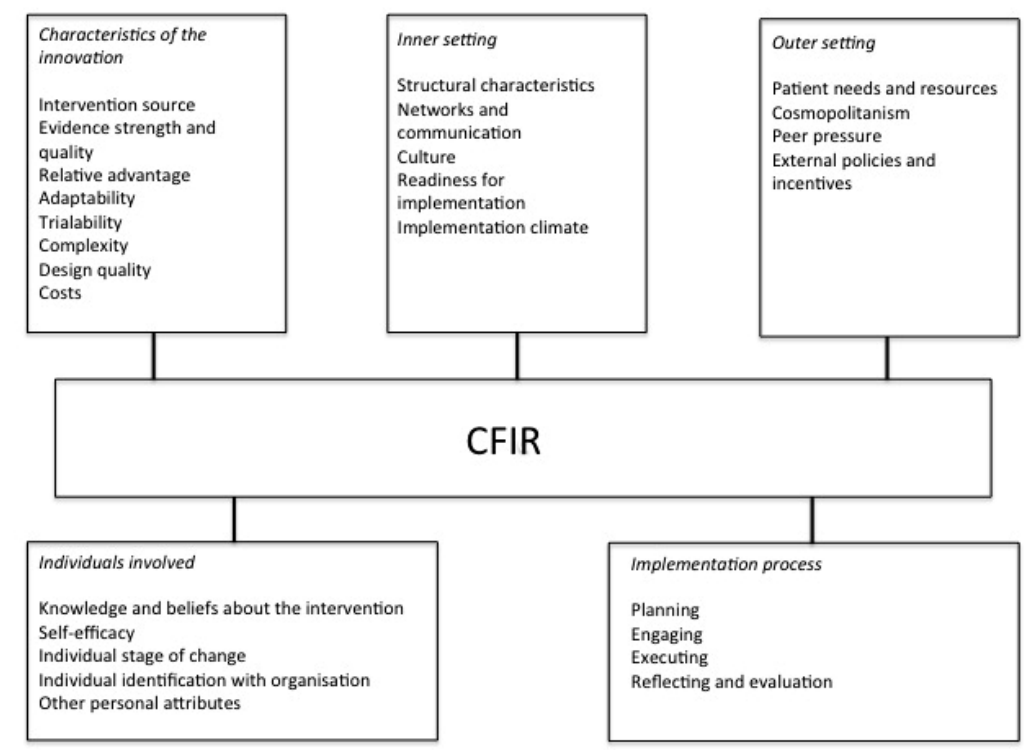

Figure 1: CFIR-Model: Damschroder et al, 2009

\section{Discussion}

This paper describes the protocol of a mixed methods observational study to gain more insight into the concept of sustainability and its determinants. Two health care innovations were implemented in a selection of Dutch hospitals three to six years ago, using different implementation strategies. A short stay programme for breast cancer surgery was implemented in four early adopter hospitals by means of a hospital tailored implementation strategy, and the Enhanced Recovery After Surgery programme in colonic surgery was implemented in 33 hospitals by means of a generic implementation strategy. As a result of these efforts, both cases showed improved short-term results in terms of hospital length of stay. Currently, it is unclear to what level these innovative programmes and their results have been sustained. In the current study, the level of consolidation of the implemented programmes will be assessed in fourteen hospitals in the Netherlands; four hospitals will participate in the breast cancer surgery case and ten hospitals will participate in the colonic surgery case.

\section{Strengths and limitations of the study}

This study will specifically and systematically address the sustainability of two health care innovations three to six years following two different implementation strategies. Regarding the breast cancer case, research on sustainability is not limited to long-term effects of the implementation strategy, but also comprises policy cost-effectiveness. In 
the primary study, a policy cost-effectiveness analysis on the short stay programme was performed. As a consequence of e.g. changes in programme elements or case-mix, the current cost-effectiveness may be different. A recalculation of the cost-effectiveness will be conducted which will partly be based on the detailed cost- and effectiveness data collected during the primary study. Another strength is the possibility to explore perceived determinants of sustainability from two different implementation strategies. Both programmes have been implemented in a multidisciplinary hospital setting. By studying these two different programmes, a large pool of medical specialists as well as other professionals can be approached. As such, this study will offer a multi-disciplinary perspective on sustainability and its determinants. We have chosen for the Consolidated Framework Implementation Research (CFIR) model to examine the determinants and the concept of sustainability. This framework is based on several scientific theories and existing models and considers a wide scope of possible determinants of sustainability.

In this study a mixed method approach will be applied, combining quantitative data with qualitative data. In the quantitative analyses we will examine whether the implementation results have been sustained, while in the qualitative analyses we will examine the professionals view on possible determinants of sustainability. The determinants of sustainability will be explored by means of semi-structured interviews with stakeholders of the health care processes and external change agents. This study is not aimed to analyse independent determinants in a quantitative manner by means of a multivariate regression model because we currently lack well-defined hypotheses on what are the most important determinants of sustainability. In this study we therefore also chose for qualitative methods. Furthermore, the outcome and care process data will be extracted from patient files. The practical advantage is that the participating professionals are not claimed for intensive data collection by means of completing case record forms (CRFs). Also, by auditing the files of the last 40 breast cancer patients operated, and of the last 30 colonic surgery patients, a Hawthorne effect will be prevented. This is the phenomenon that a team is improving its performance due to awareness of being monitored.

This study has also some limitations. The generalisability of our quantitative results regarding sustainability will be limited due to the hospital selection. An analysis of sustainability of the ERAS programme in colonic surgery including all 33 participating implementation hospitals would have been optimal. Although the ten hospitals were selected based on carefully chosen criteria, it cannot be ruled out that some of the other hospitals that initially were not successful in achieving the ERAS programme goals, continued their activities and ultimately implemented the protocol successfully. The evaluation of the effects of the short stay programme for breast cancer surgery after implementation will be conducted among the same hospitals as during the primary study. However, these early adopter hospitals may not fully represent Dutch practice regarding breast cancer surgery. The analysis of sustainability is based on extracting data from 
existing data files. Although a Hawthorne effect is thus prevented, a potential limitation is that some data of interest for this study may not be properly recorded.

Not much is known about long-term effects of implementation strategies and the determinants of sustainability. In this study, we perform an analysis on sustainability and we will try to shed light on potentially relevant attributes of sustainable change. We will build on previous work and aim to expand the knowledge regarding sustainability and its determinants. The results of this study will be relevant for researchers, implementation experts, health care practitioners and policy makers when making decisions about implementation and sustainability resource allocation. 


\section{References}

1. Grol R, Wensing M. What drives change? Barriers to and incentives for achieving evidence-based practice. Med J Aust. 2004 Mar 15;180(6 Suppl):S57-60.

2. Wilson KD, Kurz RS. Bridging implementation and institutionalization within organizations: proposed employment of continuous quality improvement to further dissemination. J Public Health Manag Pract. 2008 Mar-Apr;14(2):109-16.

3. Stetler CB, McQueen L, Demakis J, Mittman BS. An organizational framework and strategic implementation for system-level change to enhance research-based practice: QUERI Series. Implementation science IS. 2008;3:30

4. Grimshaw JM, Eccles MP, Walker AE, Thomas RE. Changing physicians' behavior: what works and thoughts on getting more things to work. J Contin Educ Health Prof. 2002 Fall;22(4):237-43.

5. Schuster MA, McGlynn EA, Brook RH. How good is the quality of health care in the United States? Milbank Q. 1998;76(4):517-63, 09. PubMed PMID: 9879302. Epub 1999/01/08. eng.

6. Grol R, Grimshaw J. From best evidence to best practice: effective implementation of change in patients' care. Lancet. 2003 Oct 11;362(9391):1225-30.

7. Grimshaw J, Eccles M, Tetroe J. Implementing clinical guidelines: current evidence and future implications. J Contin Educ Health Prof. 2004 Fall;24 Suppl 1:S31-7.

8. Baker R, Camosso-Stefinovic J, Gillies C, Shaw EJ, Cheater F, Flottorp S, et al. Tailored interventions to overcome identified barriers to change: effects on professional practice and health care outcomes. Cochrane Database Syst Rev. 2010 (3):CD005470.

9. van Bokhoven MA, Kok G, van der Weijden T. Designing a quality improvement intervention: a systematic approach. Quality \& safety in health care. 2003 Jun;12(3):215-20.

10. Sculpher M. Evaluating the cost-effectiveness of interventions designed to increase the utilization of evidence-based guidelines. Fam Pract. 2000 Feb;17 Suppl 1:S26-31.

11. Shediac-Rizkallah MC, Bone LR. Planning for the sustainability of community-based health programs: conceptual frameworks and future directions for research, practice and policy. Health Educ Res. 1998 Mar;13(1):87-108.

12. Scheirer MA. Is Sustainability Possible? A Review and Commentary on Empirical Studies of Program Sustainability. American Journal of Evaluation. 2005 September 2005;26(3):27.

13. Pluye P, Potvin L, Denis JL, Pelletier J. Program sustainability: focus on organizational routines. Health Promot Int. 2004 Dec;19(4):489-500.

14. Duckers ML, Wagner C, Vos L, Groenewegen PP. Understanding organisational development, sustainability, and diffusion of innovations within hospitals participating in a multilevel quality collaborative. Implementation science : IS. 2011;6(1):18.

15. Gruen RL, Elliott JH, Nolan ML, Lawton PD, Parkhill A, McLaren CJ, et al. Sustainability science: an integrated approach for health-programme planning. Lancet. 2008 Nov 1;372(9649):1579-89.

16. Rabin BA, Brownson RC, Haire-Joshu D, Kreuter MW, Weaver NL. A glossary for dissemination and implementation research in health. J Public Health Manag Pract. 2008 Mar-Apr;14(2):117-23.

17. NHS. Sustainability and its relationship with spread and adoption, General improvement skills. Coventry, UK: Improvement Leaders' guide; 2007

18. Wiltsey Stirman S, Kimberly J, Cook N, Calloway A, Castro F, Charns M. The sustainability of new programs and innovations: a review of the empirical literature and recommendations for future research. Implementation science : IS. 2012;7:17.

19. de Kok M, Dirksen CD, Kessels AG, van der Weijden T, van de Velde CJ, Roukema JA, et al. Costeffectiveness of a short stay admission programme for breast cancer surgery. Acta Oncol. 2010 Apr;49(3):338-46.

20. de Kok M, Frotscher CN, van der Weijden T, Kessels AG, Dirksen CD, van de Velde CJ, et al. Introduction of a breast cancer care programme including ultra short hospital stay in 4 early adopter centres: framework for an implementation study. BMC Cancer. 2007;7:117 


\section{SUSTAINABILITY OF HEALTH CARE INNOVATIONS (PROTOCOL)}

21. de Kok M, Scholte RW, Sixma HJ, van der Weijden T, Spijkers KF, van de Velde CJ, et al. The patient's perspective of the quality of breast cancer care. The development of an instrument to measure quality of care through focus groups and concept mapping with breast cancer patients. Eur J Cancer. 2007 May;43(8):1257-64.

22. de Kok M, van der Weijden T, Kessels A, Dirksen C, van de Velde C, Roukema J, et al. Implementation of an ultra-short-stay program after breast cancer surgery in four hospitals: perceived barriers and facilitators. World journal of surgery. 2008 Dec;32(12):2541-8

23. Fearon KC, Ljungqvist $\mathrm{O}$, Von Meyenfeldt $\mathrm{M}$, Revhaug $\mathrm{A}$, Dejong $\mathrm{CH}$, Lassen $\mathrm{K}$, et al. Enhanced recovery after surgery: a consensus review of clinical care for patients undergoing colonic resection. Clin Nutr. 2005 Jun;24(3):466-77.

24. Maessen J, Dejong CH, Hausel J, Nygren J, Lassen K, Andersen J, et al. A protocol is not enough to implement an enhanced recovery programme for colorectal resection. Br J Surg. 2007 Feb;94(2):224-31.

25. Maessen JMC, Dejong CH, Kessels AG, von Meyenfeldt MF. Length of stay: an inappropriate readout of the success of enhanced recovery programs. World journal of surgery. 2008 Jun;32(6):971-5.

26. Oostenbrink JB, Bouwmans CAM, Koopmanschap MA, en Rutten FFH. Handleiding voor kostenonderzoek, methoden en standaard kostprijzen voor economische evaluaties in de gezondheidszorg: College voor zorgverzekeringen. Geactualiseerde versie 2004.

27. Severens JL. Value for money of changing healthcare services? Economic evaluation of quality improvement. Qual Saf Health Care 2003, 12:366-371

28. EuroQol--a new facility for the measurement of health-related quality of life. The EuroQol Group. Health Policy. 1990 Dec;16(3):199-208.

29. Efron B. Introduction to the bootstrap: Chapman \& Hall: New York, USA; 1993.

30. Damschroder LJ, Aron DC, Keith RE, Kirsh SR, Alexander JA, Lowery JC. Fostering implementation of health services research findings into practice: a consolidated framework for advancing implementation science. Implementation science : IS. 2009;4:50.

31. Rogers E. Diffusion on innovations. 5th ed. New York: NY: Free Press; 2003.

32. Damschroder LJ, Hagedorn HJ. A guiding framework and approach for implementation research in substance use disorders treatment. Psychol Addict Behav. 2011 Mar 28.

33. Simpson DD, Flynn PM. Moving innovations into treatment: A stage-based approach to program change. J Subst Abuse Treat. 2007 Sep;33(2):111-20.

34. Greenhalgh T, Robert G, Macfarlane F, Bate P, Kyriakidou O. Diffusion of innovations in service organizations: systematic review and recommendations. Milbank Q. 2004;82(4):581-629. 


\section{CHAPTER 2}

\section{Additional file 1 Interview themes for sustainability}

What is your vision on the current results?

- Factors

Who was involved during the implementation process?

To what extent did these roles change?

Leader

Are there any changes made in the programme?

- Protocol adherence

Are there any structural changes and activities executed after the implementation? (costs)

- Other investments

- Strategy

- Monitoring

Is there regular communication and feedback between involved professionals?

- Networks and structure

Is there an innovative culture within the hospital?

Are all programme elements understood by the involved professionals?

Were there changes on patient level?

- Patient needs

- Case mix

Do external policy and trends have an influence?

- Transparency performance indicators

What is the influence of other hospitals?

- Peer pressure

- Communication

Which multidisciplinary team members are involved?

What is the level of efficacy of the team members?

What are their support and beliefs about the programme?

What is the level of knowledge and training? 


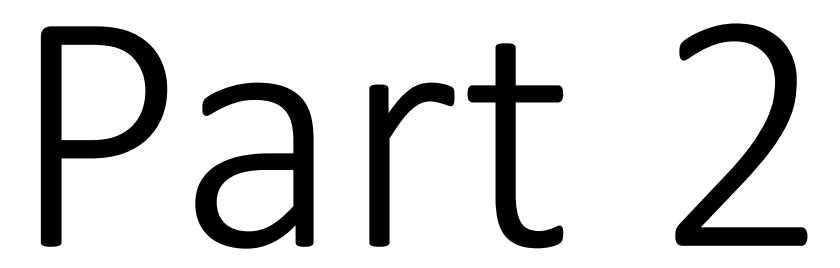

Quantitative analyses of the level of

sustainability 



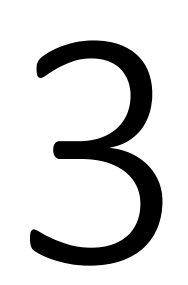

\section{Sustainability of short stay for breast cancer} surgery in early adopter hospitals

Stephanie M C Ament, F Gillissen, J M C Maessen, C D Dirksen, A V R J Bell,

Y L J Vissers, T van der Weijden, M F von Meyenfeldt

Published: The Breast, Volume(s) 23, 01-april-2014, Pages 429-434 


\section{CHAPTER 3}

\section{Abstract}

\section{Background}

Breast cancer care is a dynamic field and is always changing. Innovations are constantly being developed and introduced to improve quality and efficiency of care. It is highly desirable to maintain achieved quality improvements. However, research shows that it is difficult to sustain change as people tend to return to old routines. Between 2005 and 2007 a short stay programme for breast cancer surgery was successfully implemented in early adopter hospitals. The current study evaluates the sustainability of this success five years following implementation.

\section{Methods}

A retrospective audit of 160 consecutive patients undergoing breast cancer surgery was performed five years following implementation of short stay. Sustainability of the primary implementation success was assessed by measuring the primary outcome, the proportion of patients treated in short stay (day or 24 hour admission), and compliance to the key recommendations to facilitate short stay for breast cancer surgery. Data were analysed on overall and on hospital level.

\section{Results}

The total proportion of patients treated in short stay was $82 \%$ (hospital $183 \%$, hospital $278 \%$, hospital $387 \%$, hospital $480 \%$ ) after five years follow-up, which was comparable to the proportion in short stay directly after implementation $(p=0.938)$. Overall compliance to the key recommendations to facilitate short stay for breast cancer surgery increased from 65\% directly after implementation to $78 \%$ five years after implementation. The current study showed that type of surgery was a predictive factor for successful short stay treatment in the real world.

\section{Conclusion}

This study shows that short stay for breast cancer surgery was successfully sustained in early adopter hospitals five years following implementation. 


\section{Introduction}

Breast cancer care is constantly advancing and adapting to new findings and changing needs. As a result, treatments are improving and care is becoming more complex, specialised and given in a multidisciplinary setting [1]. However, further quality and efficiency improvement in breast cancer care is needed, given the fact that the breast cancer incidence is still raising worldwide as a consequence of the ageing population and increased mammographic screening [2]. As the majority of these patients undergo surgery, the growing breast cancer incidence will continue to put pressure on hospital care. Reducing hospital length of stay is one way to improve efficiency. From 2001 to 2002, a short stay programme for breast cancer surgery was introduced in a pilot study at the Maastricht University Medical Centre (MUMC). This resulted in a significant reduction in mean length of hospital stay. Recently, other implementation studies confirmed that short stay for breast cancer surgery can be effective, efficient and feasible while enhancing the quality of care [3-7]. Unfortunately, these studies addressed only shortterm effects of implementing short stay for breast cancer surgery. Currently, it is unknown whether achieved implementation results of short stay for breast cancer surgery are maintained in the real world.

Between 2005 and 2007, short stay for breast cancer surgery was successfully introduced in early adopting hospitals in the Netherlands. The aim of the implementation project was to increase the proportion of patients treated in short stay while maintaining the quality of care as perceived by patients. The short stay programme comprised streamlining the multidisciplinary pathway and improving patient information [8]. The short stay programme was implemented by means of a hospital tailored implementation strategy, meaning that the strategy was shaped to the specific circumstances and needs of the hospital (staff). Initial implementation results showed that the implementation strategy was successful. The implementation study showed an overall increase from $45 \%$ to $82 \%$ in uptake of short stay without a negative effect on quality of life or patient satisfaction, number of readmissions and complications [9]. Moreover, mean societal costs decreased €955,- per patient treated in short stay, compared with patients treated in care as usual [6].

Typically, introduction of innovations in daily practice is project based with an important role of the change agent, who may be an external stakeholder such as an academic research group. After the implementation phase, the challenge is to internalise and normalise the change and to sustain achieved quality improvements $[10,11]$. Sustaining change means maintaining quality improvements for a longer period of time [12, 13]. Sustaining quality improvement, however, is not self-evident as it is a dynamic and complex process [14-17]. Embedding multidisciplinary treatment of patients in short stay admission needs, besides restructuring care, for example also a structural change in mindset by professionals and patients. Returning to the old routines would waste the invested money and time while care would remain inefficient. Therefore, a long-term 


\section{CHAPTER 3}

approach of holding implementation gains is needed when embedding change. Unfortunately, not much research has been conducted regarding the level of sustainability once an innovation has been implemented [16].

The aim of this paper is to evaluate the sustainability of a short stay programme for breast cancer surgery after initially achieved implementation successes. The sustainability evaluation is illustrated by an implementation case focused on short stay for breast cancer surgery in early adopting hospitals. More insight in sustained implementation success in the real world is useful for professionals, managers and researchers who decide to implement innovations.

\section{Materials and methods}

\section{Design}

Performance regarding breast cancer surgery care was measured prospectively as part of the primary implementation study before implementation (2005-2006, preimplementation: PRE group) and directly after implementation (2006-2007, early postimplementation: POST group) [8]. In the present study, sustainability of a short stay programme for breast cancer surgery was evaluated using a follow-up measurement five years following implementation (LATE POST group). The retrospective audit of patient records of the LATE POST group was performed between May 2012 and December 2012. The study protocol has been published elsewhere [18] and the Medical Research Ethics Committee of the University of Maastricht has granted approval, METC 11-4015.10 .

\section{Participants}

\section{Hospitals}

The same hospitals which participated in the primary implementation study were included in this study, covering four main organisational hospital settings in the Netherlands.

\section{Patients}

The same in- and exclusion criteria were used as in the primary implementation study. Patients who were over 18 years old, diagnosed with breast cancer and had undergone breast surgery were eligible. Patient records of the last consecutive 160 patients who had been scheduled for breast cancer surgery (40 patients per centre) were audited, the same number as the PRE and POST measurement. 
Variables for sustainability evaluation

There is no uniform definition or method to evaluate the sustainability of health care innovations [16]. We defined sustainability in the study protocol as: "Sustainability of change exists when a newly implemented innovation continues to deliver the achieved benefits over a longer period of time, certainly does not return to the usual processes and becomes 'the way things are done around here', even after the implementation project is no longer actively carried out, until a better innovation comes along" [18]. Sustainability of a short stay programmefor breast cancer surgery was assessed with respect to two sustainability measures: (1) continuation of delivering the achieved benefits in terms of achieved overall proportion of patients treated in short stay and (2) continuation of overall adherence to the key recommendations of the programme to facilitate short stay for breast cancer surgery.

\section{Proportions of patients treated in short stay}

The same outcome was used as in the primary implementation study (box 1), namely the proportion of patients treated in short stay. Short stay admission comprises treating patients in day admission or in overnight admission ( $24 \mathrm{~h}$ admission). The aim of the primary implementation study was to increase the proportion of patients treated in short stay. In the current study, the primary outcome was assessed as sustained if the overall proportion of patients treated in short stay did not decrease compared to the POST group. Also, the proportion of patients treated in day admission, the proportion of patients planned in short stay, the duration of hospital admission, and the reasons for inpatient care were determined.

\section{Key recommendations to facilitate short stay}

Thirteen key recommendations had been developed and implemented in the primary implementation study. The key recommendations were aimed to improve the quality and efficiency of the breast cancer surgery care process. The rationale behind the key recommendations is that a more streamlined care process fits and complements the short stay approach for breast cancer surgery. In this evaluation a selection of the key recommendations was made based on two criteria [8]: (1) adherence to the key recommendation was measured in the primary implementation study and (2) measurement of adherence to the key recommendation was possible by auditing patient files (additional file 1). The following six key recommendations were checked in this evaluation:

- The interval between referral and first visit to the breast unit is five working days or less

- Treatment is discussed in a preoperative multidisciplinary meeting

- The interval between diagnostic tests and informing patients about the results is five working days or less 


\section{CHAPTER 3}

- The interval between the decision to operate and surgery is fifteen working days or less

- The general practitioner is informed about diagnosis, treatment plan and potential side-effects prior to surgery

- The breast nurse stays in contact with the patient during postoperative phase (phone consultation)

The adherence to the key recommendations was considered as sustained if the overall adherence proportions in the LATE POST group did not decrease in comparison with the post implementation results.

\section{Statistical analysis}

Differences in patient characteristics and primary and secondary outcomes between the POST and the LATE POST group were compared using the Mann-Withney $U$ test for continuous variables and Chi-squared test for categorical variables. Proportions of patients treated in short stay, treated in day admission and planned in short stay were compared between the POST and the LATE POST group using a Chi-squared test. A logistic regression analysis was performed to determine whether age group and type of surgery had influence on the proportion of patients treated in short stay in the LATE POST group. All statistical analyses were performed using SPSS 20.0 (SPSS Inc. Chicago, Illinois, USA). $\mathrm{P}<0.05$ was considered statistically significant.

\section{Results}

\section{Patient characteristics}

In total, data of 484 female breast cancer patients were used in this sustainability evaluation. In the PRE group, POST group and the LATE POST group 161, 163 and $160 \mathrm{pa-}$ tients were included respectively. Patient characteristics are presented in table 1, patient characteristics on hospital level are presented in table 2. Mean age and the proportion of patients aged 65 years and older were significantly higher in the LATE POST group in comparison with the POST group $(p<0.05)$. On hospital level, mean age and the proportion of patients aged 65 years and older were significantly higher in hospitals 1 and 4 in the LATE POST group in comparison with the POST group $(p<0.05)$. More mastectomies $(p<0.05)$ and more immediate reconstructions $(p<0.05)$ were observed in the LATE POST group compared with the POST group. On hospital level, more mastectomies $(p<0.05)$ were observed in hospitals 1 and 2 and more immediate reconstructions $(p<0.05)$ were observed in hospital 1 in the LATE POST group compared with the POST group. 
SUSTAINABILITY OF SHORT STAY FOR BREAST CANCER SURGERY

Table 1. Patient characteristics of the PRE, POST and LATE POST group

\begin{tabular}{|c|c|c|c|c|}
\hline & & $\begin{array}{c}\text { PRE } \\
n=161\end{array}$ & $\begin{array}{c}\text { POST } \\
n=163\end{array}$ & $\begin{array}{c}\text { LATE POST } \\
n=160\end{array}$ \\
\hline \multirow[t]{2}{*}{ Age (years) } & mean (SD) & $55.3(11,5)$ & $56.8(10.8)$ & $61.5(13.2)^{*}$ \\
\hline & 65 years and older (\%) & $34(21)$ & $34(21)$ & $73(46)^{*}$ \\
\hline \multirow[t]{2}{*}{ Type of surgery (\%) } & Breast conserving & $88(55)$ & $109(67)^{*}$ & $75(47)^{*}$ \\
\hline & Mastectomy & $73(45)$ & $54(33)^{*}$ & $85(53)^{*}$ \\
\hline Immediate reconstruction (\%) & Yes & $0(0)$ & $0(0)$ & $12(8)^{*}$ \\
\hline
\end{tabular}

Table 2. Patient characteristics of the POST and LATE POST group per hospital

\begin{tabular}{|c|c|c|c|}
\hline & & POST & LATE POST \\
\hline Hospital 1 & & $n=40$ & $n=40$ \\
\hline \multirow[t]{2}{*}{ Age (years) } & mean (SD) & $57.4(9.73)$ & $64.5(12.1)^{*}$ \\
\hline & 65 years and older (\%) & $5(13 \%)$ & $22(55 \%)^{*}$ \\
\hline \multirow[t]{3}{*}{ Type of surgery (\%) } & Breast conserving & $21(53 \%)$ & $10(25 \%)^{*}$ \\
\hline & Mastectomy & $19(48 \%)$ & $30(75 \%)^{*}$ \\
\hline & Immediate reconstruction (\%) & $0(0 \%)$ & $11(28 \%)^{*}$ \\
\hline Hospital 2 & & $n=50$ & $n=40$ \\
\hline \multirow[t]{2}{*}{ Age (years) } & mean (SD) & $57.6(11.8)$ & $60.1(15.2)$ \\
\hline & 65 years and older (\%) & $14(28 \%)$ & $15(38 \%)$ \\
\hline \multirow[t]{3}{*}{ Type of surgery (\%) } & Breast conserving & $32(64 \%)$ & $14(35 \%)^{*}$ \\
\hline & Mastectomy & $18(36 \%)$ & $26(65 \%)^{*}$ \\
\hline & Immediate reconstruction (\%) & $0(0 \%)$ & $1(2,5 \%)$ \\
\hline Hospital 3 & & $n=34$ & $n=40$ \\
\hline \multirow[t]{2}{*}{ Age (years) } & mean (SD) & $58.2(10.8)$ & $61.8(13.8)$ \\
\hline & 65 years and older (\%) & $10(29 \%)$ & $17(43 \%)$ \\
\hline \multirow[t]{3}{*}{ Type of surgery (\%) } & Breast conserving & $26(77 \%)$ & $28(70 \%)$ \\
\hline & Mastectomy & $8(24 \%)$ & $12(30 \%)$ \\
\hline & Immediate reconstruction (\%) & $0(0 \%)$ & $0(0 \%)$ \\
\hline Hospital 4 & & $n=39$ & $n=40$ \\
\hline \multirow[t]{2}{*}{ Age (years) } & mean (SD) & $53.8(10.2)$ & $61.1(11.4)^{*}$ \\
\hline & 65 years and older (\%) & $5(13 \%)$ & $19(48 \%)^{*}$ \\
\hline \multirow[t]{3}{*}{ Type of surgery (\%) } & Breast conserving & $30(77 \%)$ & $23(58 \%)$ \\
\hline & Mastectomy & $9(23 \%)$ & $17(43 \%)$ \\
\hline & Immediate reconstruction (\%) & $0(0 \%)$ & $0(0 \%)$ \\
\hline
\end{tabular}




\section{CHAPTER 3}

\section{Proportions of patients treated in short stay}

The overall proportion of patients treated in short stay did not change and was maintained at $82 \%$ in the LATE POST group compared with the POST group ( $p=0.938$ ) (figure 1). The proportion of patients treated in short stay increased from $74 \%$ to $78 \%$ in centre 2 and increased from $73 \%$ to $87 \%$ in centre 3 . In centre 4 , the proportion of patients treated in short stay decreased from $87 \%$ to $80 \%$. The proportion of patients treated in short stay decreased from $95 \%$ to $83 \%$ in centre 1 .

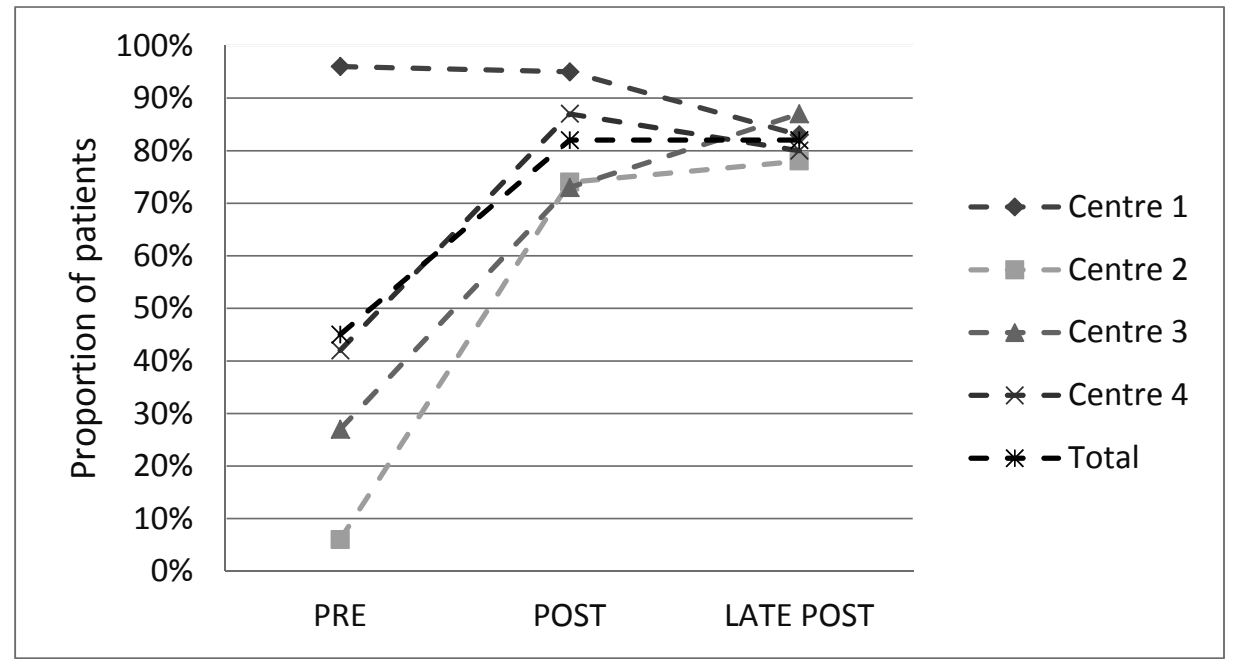

Figure 1. Proportion of breast cancer surgery patients treated in short stay in the PRE, POST and LATE POST group

The overall proportion of patients treated in real day admission decreased from $33 \%$ in the POST group to $28 \%$ in the LATE POST group ( $p=0.604$ ) (figure 2). In centre 2 and 3 the proportion of patients discharged on the day of surgery increased (from $2 \%$ to $8 \%$ in centre 2 and from 35\% to 50\% in centre 3). The proportion of patients treated in day admission decreased in centre 1 and 4 in the LATE POST group compared with the POST group (from $60 \%$ to $28 \%$ in centre 1 and from $44 \%$ to $30 \%$ in centre 4 ). Mean length of hospital stay was 0,9 day in the LATE POST group (SD 0,8) and was 1,1 day (SD 2,9) in the POST group and was not significantly different $(p=0.772)$. 


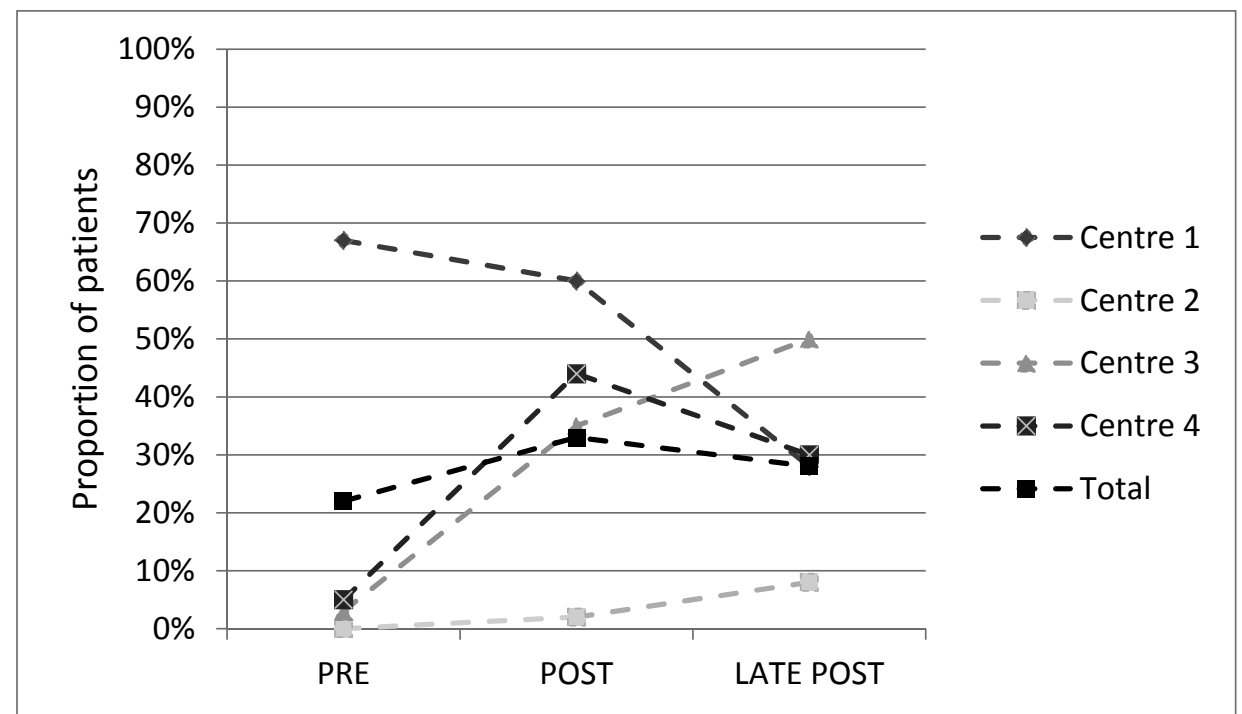

Figure 2. Proportion of breast cancer surgery patients treated in day admission in the PRE, POST and LATE POST group

The overall proportion of patients planned in short stay slightly decreased from $95 \%$ in the POST group to $93 \%$ in the LATE POST group ( $p=0.255)$. The proportion of patients planned in short stay decreased in three out of four centres. The proportion of patients planned in short stay increased in centre 4. There were no data available of the PRE group. Reasons for a delayed discharge in the LATE POST group were related to the patient's wellbeing (eleven patients). The reason for delayed discharge was missing for thirteen patients. Reasons for inpatient planning in the LATE POST were patient's wellbeing (six patients) and a lack of informal care support during the first 24 hours after discharge (three patients). One patient in the LATE POST group was not planned in short stay due to breast cancer surgery in combination with an immediate reconstruction.

\section{Key recommendations to facilitate short stay}

Overall compliance with the key recommendations increased from $65 \%$ in the POST group to $78 \%$ in the LATE POST (table 3). The highest adherence (100\%) was observed in key recommendation 'Treatment is discussed in a preoperative multidisciplinary meeting' and the lowest adherence (15\%) was observed in key recommendation 'breast nurse stays in contact with the patient after short stay (phone consultation)'. Compliance increased in hospital 2 and 3, hospital 1 showed decreased compliance and hospital 4 showed equal compliance results. 
Table 3. Adherence to the key recommendations to facilitate short stay for breast cancer surgery in the PRE, POST and LATE POST group (excluding missing values)

\begin{tabular}{|c|c|c|c|}
\hline & PRE & POST & LATE POST \\
\hline Treatment is discussed in a preoperative multidisciplinary meeting & $139(86)$ & $154(95)$ & $156(100)$ \\
\hline $\begin{array}{l}\text { The interval between referral and first visit to the breast unit is five } \\
\text { working days or less }\end{array}$ & $16(37)$ & $45(61)$ & $109(84)$ \\
\hline $\begin{array}{l}\text { The interval between diagnostic tests and informing patients about their } \\
\text { results is five working days or less }\end{array}$ & $99(62)$ & $105(64)$ & $133(90)$ \\
\hline $\begin{array}{l}\text { The interval between the decision to operate and surgery is fifteen } \\
\text { working days or less }\end{array}$ & $144(89)$ & $131(80)$ & $128(80)$ \\
\hline $\begin{array}{l}\text { The general practitioner is informed about diagnosis, treatment plan } \\
\text { potential side-effects prior to surgery }\end{array}$ & $118(73)$ & $123(76)$ & $156(98)$ \\
\hline $\begin{array}{l}\text { The breast nurse stays in contact with the patient after short stay (phone } \\
\text { consultation) }\end{array}$ & $11(7)$ & $19(12)$ & $24(15)$ \\
\hline Overall adherence (\%) & 59 & 65 & 78 \\
\hline
\end{tabular}

\section{Predictors of treatment in short stay}

Logistic regression analysis showed that undergoing breast conserving surgery (OR 95\% Cl 4.3 1.6-11.2) was associated with a higher probability of treatment in short stay. On hospital level, undergoing breast conserving surgery was shown to be associated with a higher probability of short stay in hospital 4 (OR 95\% Cl 19.6 1.9-202.5) in the LATE POST group. Furthermore, patients undergoing immediate reconstruction were less likely to be treated in short stay in hospital 1 (OR 95\% Cl $0.090 .01-0.97$ ) in the LATE POST group.

\section{Discussion}

Breast cancer surgery is increasingly performed in short stay and prior research has shown that implementation of short stay for breast cancer surgery was successful [5]. However, these studies were focused on early implementation successes. The current study focused on the long-term achievement of implementation of breast cancer surgery performed in short stay. Our results showed that the achieved benefit, in terms of the overall proportion of patients treated in short stay, was fully sustained. In addition, the adherence to the key recommendations seems to have normalised in the LATE POST group. Therefore, the current study confirms that short stay for breast cancer surgery was sustained and the quality of the care process was even further improved in the early adopter hospitals.

This evaluation showed that sustainability of short stay is possible in a real world setting. However, relevant practice variation between hospitals was observed regarding the proportions of patients treated in day admission. This may be explained by the type of surgery at hospital level and by possible modifications of the application of short stay 
admission on centre level over a longer period of time [19]. Previous research showed that age, breast conserving surgery, having children and having a job are predictive factors for short stay admission for breast cancer surgery [9]. The current study showed that undergoing breast conserving surgery was a predictive factor for successful short stay treatment in the real world. In hospital 1, the case mix was significantly different in the LATE POST group compared to the POST group as more patients received immediate reconstruction and patients were older. This may explain the decreased proportion of patients treated in short stay in hospital 1 . However, the overall proportion of patients treated in short stay was fully maintained at $82 \%$, despite a significantly higher age and significantly more mastectomies in the LATE POST group compared with the POST group. This result highlights full embedding of short stay five years following implementation. In addition, practice variation between hospitals regarding the proportions of patients treated in short stay has been diminished. This may imply that the application of short stay for breast cancer surgery became more normalised and balanced after years of experience. Yet, short stay for breast cancer surgery is not applicable to all patients. Patients' physiology and preferences are still taken into account five years following implementation. Clearly, patient specific decisions must be made to guarantee the quality and safety of breast cancer surgical care. Furthermore, the key recommendation 'breast nurse stays in contact with the patient after short stay (phone consultation)' showed a relatively low adherence rate. This element was perceived as a facilitating factor for short stay by surgeons and breast nurses in participating hospitals before implementation. However, in reality this key recommendation seems not always necessary for short stay for breast cancer surgery.

Sustainability of quality improvement is a dynamic process [20, 21]. Actual performance may have been influenced by ongoing changes and developments in breast cancer surgical care. For example, the national context regarding quality of breast cancer care in the Netherlands has been changing. National registration of breast cancer care performance to increase quality of care has taken its position over the years. Verbeek et al. showed that registration and benchmarking contributed to an increase in quality of breast cancer care between 2002 and 2008 in the Midwestern part of the Netherlands [23]. As a consequence of the co-occurrence of macro level advances, adherence to the key recommendations of short stay for breast cancer surgery may have been further improved between 2008 and 2012. Moreover, hospital length of stay for all diagnoses is decreasing worldwide. In the Netherlands average hospital length of stay for all causes decreased from 8.5 days in 2000 to 5.6 days in 2009 [24]. More care is also delivered in outpatient settings instead of inpatient settings [25]. As a result of a general decrease in hospital length of stay for all causes, sustainability of the short stay performance could have been more influenced by these developments than by the primary implementation efforts. However, the national mean hospital length of stay for breast cancer surgery was 1.52 (sd: 1.52, IQR 25-75: 1) and 66\% of all patients was treated in day admission or was discharged the day after surgery in 2011 (Netherlands Cancer Registry). 
Clearly, the early adopter hospitals that participated in the primary implementation study perform better than the national results. Being an early adopter hospital may have been facilitators for these long-term results. Independently from changes at the national level following the implementation, practice at the local level may have changed as well [26]. For example, new insights and advances in practice, such as immediate reconstruction, were introduced in some centres. This may also have influenced the sustainability of short stay.

A strength of the current study was the use of a sustainability work definition focusing on maintaining achieved benefits and continuation of adherence. Currently, long-term implementation research is fragmented and a high proportion of studies in the field of sustainability do not use a work definition [16]. Sustainability was primarily assessed on patient level instead of on hospital level due to the low number of patients per hospital. Information on hospital level was also presented, but was not included in our final conclusion. It is possible that the short stay admission rate may be different in a next audit due to changes in context or adaptations within practices as breast cancer care is continuously changing. A strength of evaluating sustainability of short stay by using an audit is that all eligible patients are included. Furthermore, this audit being retrospective prevented a Hawthorne effect to occur. A Hawthorne effect is the phenomenon that a team is improving its performance due to awareness of being monitored. Moreover, the audit was performed by two researchers (FG and SA), who were not involved in the primary implementation study. This resulted in a more independent observation. On the other hand, we compared data which were collected using different techniques. Thus, retrospective audit data (LATE POST group) were compared to the prospectively collected data in the POST group. This may have contributed to the significant differences in age and type of surgery between these groups. For example, patients who did not participate in the primary implementation study may have been older than the actually included patients as it can be assumed that the chance for a Hawthorne effect may be higher in the prospective group. In the retrospective audit, all consecutive patients were included thus limiting patient selection bias, and a more truthful view of "how things are done around here".

This study made an attempt to gain new insight in sustainability of initially successful implementation of an innovation which is used by a multidisciplinary team in the cure setting. Currently, not much sustainability research is done in this type of setting. However, the path to sustainability may be different in other target locations [27]. The generalisability may therefore be limited to other health care settings. Notably, hospitals which were selected in the primary implementation study were early adopting hospitals in breast cancer surgery care. Being recognised as an early adopter may have been a facilitator for sustaining change. These early adopter hospitals may not fully represent Dutch practice regarding innovation in the field of breast cancer care. As a consequence, external validity may be limited due to a hospital selection bias. Furthermore, short stay was introduced by means of an implementation strategy which was 
tailored to the specific needs of each hospital. The effectiveness of tailored implementation strategies in improving clinical practice is unknown, though they seem promising [28] [29]. Thus, current short stay results could be different if a large-scale non-tailored implementation strategy had been used.

Determinants of primary implementation results are not necessarily the same as for sustainability results. Therefore, more research is needed to understand the paths to sustainability of health care innovations. For example, the role of being an early adopter or the use of the tailored implementation strategy must be analysed. Additional qualitative research will be performed in participating hospitals and is intended to explore the determinants of maintaining early implementation successes of a complex innovation over a longer period of time [18].

\section{Conclusion}

This sustainability evaluation provided insight into long-term implementation results of a short stay programmefor breast cancer surgery in early adopter hospitals. Short stay admission for breast cancer surgery was shown to be normalised in a real world setting. Further spread of short stay is recommended to improve quality and efficiency of breast cancer care. 


\section{CHAPTER 3}

\section{References}

1. Wouters MW, Jansen-Landheer ML, van de Velde CJ. The Quality of Cancer Care initiative in the Netherlands. European journal of surgical oncology : the journal of the European Society of Surgical Oncology and the British Association of Surgical Oncology. 2010 Sep;36 Suppl 1:S3-S13.

2. Forouzanfar MH, Foreman KJ, Delossantos AM, Lozano R, Lopez AD, Murray CJ, et al. Breast and cervical cancer in 187 countries between 1980 and 2010: a systematic analysis. Lancet. 2011 Oct 22;378(9801):1461-84.

3. Weber WP, Barry M, Junqueira MJ, Lee SS, Mazzella AM, Sclafani LM. Initial experiences with a multidisciplinary approach to decreasing the length of hospital stay for patients undergoing unilateral mastectomy. European journal of surgical oncology : the journal of the European Society of Surgical Oncology and the British Association of Surgical Oncology. 2011 Nov;37(11):944-9.

4. Hainsworth AJ, Lobo CR, Williams P, Case C, Surridge F, Sharma AK, et al. '23 h Model' for breast surgery: An early experience. Breast (Edinburgh, Scotland). 2013 May 8.

5. Marla S, Stallard S. Systematic review of day surgery for breast cancer. International journal of surgery (London, England). 2009 Aug;7(4):318-23.

6. de Kok M, Dirksen CD, Kessels AG, van der Weijden T, van de Velde CJ, Roukema JA, et al. Costeffectiveness of a short stay admission programme for breast cancer surgery. Acta Oncol. 2010 Apr;49(3):338-46.

7. Mertz BG, Kroman N, Williams H, Kehlet H. Fast-track surgery for breast cancer is possible. Danish medical journal. 2013 May;60(5):A4615.

8. de Kok M, Frotscher CN, van der Weijden T, Kessels AG, Dirksen CD, van de Velde CJ, et al. Introduction of a breast cancer care programme including ultra short hospital stay in 4 early adopter centres: framework for an implementation study. BMC Cancer. 2007;7:117.

9. de Kok M, van der Weijden T, Voogd AC, Dirksen CD, van de Velde CJ, Roukema JA, et al. Implementation of a short-stay programme after breast cancer surgery. Br J Surg. 2010 Feb;97(2):189-94.

10. Scheirer MA, Dearing JW. An agenda for research on the sustainability of public health programs. American journal of public health. 2011 Nov;101(11):2059-67.

11. Rabin BA, Brownson RC, Haire-Joshu D, Kreuter MW, Weaver NL. A glossary for dissemination and implementation research in health. J Public Health Manag Pract. 2008 Mar-Apr;14(2):117-23.

12. Greenhalgh T, Robert G, Macfarlane F, Bate P, Kyriakidou O. Diffusion of innovations in service organizations: systematic review and recommendations. Milbank Q. 2004;82(4):581-629.

13. Shediac-Rizkallah MC, Bone LR. Planning for the sustainability of community-based health programs: conceptual frameworks and future directions for research, practice and policy. Health Educ Res. 1998 Mar;13(1):87-108.

14. Grol R, Grimshaw J. From best evidence to best practice: effective implementation of change in patients care. Lancet. 2003 Oct 11;362(9391):1225-30.

15. Grimshaw JM, Shirran L, Thomas R, Mowatt G, Fraser C, Bero L, et al. Changing provider behavior: an overview of systematic reviews of interventions. Med Care. 2001 Aug;39(8 Suppl 2):I12-45.

16. Wiltsey Stirman S, Kimberly J, Cook N, Calloway A, Castro F, Charns M. The sustainability of new programs and innovations: a review of the empirical literature and recommendations for future research. Imple mentation science : IS. 2012;7:17.

17. Gruen RL, Elliott JH, Nolan ML, Lawton PD, Parkhill A, McLaren CJ, et al. Sustainability science: an integrated approach for health-programme planning. Lancet. 2008 Nov 1;372(9649):1579-89.

18. Ament SMC, Gillissen F, Maessen JMC, Dirksen CD, van der Weijden T, von Meyenfeldt MF. Sustainability of healthcare innovations (SUSHI): long term effects of two implemented surgical care programmes (protocol). BMC health services research. 2012;12:423.

19. Stirman SW, Miller CJ, Toder K, Calloway A. Development of a framework and coding system for modifications and adaptations of evidence-based interventions. Implementation science : IS. 2013 Jun 10;8(1):65.

20. Rogers E. Diffusion on innovations. 5th ed. New York: NY: Free Press; 2003. 


\section{SUSTAINABILITY OF SHORT STAY FOR BREAST CANCER SURGERY}

21. Plochg T, Delnoij DM, Hoogedoorn NP, Klazinga NS. Collaborating while competing? The sustainability of community-based integrated care initiatives through a health partnership. BMC health services research. 2006;6:37.

22. Veerbeek L, van der Geest L, Wouters M, Guicherit O, Does-den Heijer A, Nortier J, et al. Enhancing the quality of care for patients with breast cancer: seven years of experience with a Dutch auditing system. European journal of surgical oncology : the journal of the European Society of Surgical Oncology and the British Association of Surgical Oncology. 2011 Aug;37(8):714-8.

23. OECD. Average length of stay in hospitals, In: Health at a Glance 2011: OECD Indicators. 2011.

24. Krystyna-Wasowicz D. Trends in day surgery in the Netherlands. Enschede: Universiteit Utrecht; 2008.

25. Greenberg CC, Lipsitz SR, Hughes ME, Edge SB, Theriault R, Wilson JL, et al. Institutional variation in the surgical treatment of breast cancer: a study of the NCCN. Annals of surgery. 2011 Aug;254(2):339-45.

26. Scheirer MA. Linking sustainability research to intervention types. American journal of public health. 2013 Apr;103(4):e73-80.

27. Baker R, Camosso-Stefinovic J, Gillies C, Shaw EJ, Cheater F, Flottorp S, et al. Tailored interventions to overcome identified barriers to change: effects on professional practice and health care outcomes. Cochrane Database Syst Rev. 2010 (3):CD005470.

28. Wensing M, Oxman A, Baker R, Godycki-Cwirko M, Flottorp S, Szecsenyi J, et al. Tailored Implementation For Chronic Diseases (TICD): a project protocol. Implementation science : IS. 2011;6:103. 


\section{CHAPTER 3}

Additional file 1

Measurement of key recommendations to facilitate short stay in breast cancer surgery in sustainability evaluation

\begin{tabular}{|c|c|c|c|}
\hline Key recommendations & $\begin{array}{c}\text { Measured in primary } \\
\text { implementation } \\
\text { study }\end{array}$ & $\begin{array}{l}\text { Measured in } \\
\text { sustainability } \\
\text { evaluation }\end{array}$ & $\begin{array}{l}\text { Measurable on } \\
\text { patient level }\end{array}$ \\
\hline Interval referral-1st visit $<5$ working days & + & + & + \\
\hline $\begin{array}{l}\text { The treatment of all patients is planned in a weekly } \\
\text { multdisciplinary meeting (MDO) }\end{array}$ & + & + & + \\
\hline Interval diagnostic tests - informing $<5$ working days & + & + & + \\
\hline Interval decision to operate - surgery $<15$ working days & + & + & + \\
\hline Home care is offered after breast cancer surgery & + & + & + \\
\hline $\begin{array}{l}\text { Breast cancer nurse informs patient about need for } \\
\text { informal care in the home situation }\end{array}$ & + & + & - \\
\hline Number of hospital visits is minimised & - & - & - \\
\hline GP is preop informed about diagn, treatment & + & + & + \\
\hline $\begin{array}{l}\text { At least one night of hospital admission is planned for } \\
\text { patients with comorbidity }\end{array}$ & - & - & + \\
\hline $\begin{array}{l}\text { Patients planned for day care are postoperatively given } \\
\text { the choice for discharge }\end{array}$ & + & - & + \\
\hline $\begin{array}{l}\text { Info given to patients about discharge is supported by } \\
\text { written info }\end{array}$ & - & + & + \\
\hline $\begin{array}{l}\text { Decisions on patient discharge are based on clear } \\
\text { guidelines }\end{array}$ & - & - & - \\
\hline $\begin{array}{l}\text { Breast cancer nurse stays in contact with the patient } \\
\text { during postoperative period }\end{array}$ & - & + & + \\
\hline
\end{tabular}




\section{4}

\section{Sustainability of an Enhanced Recovery}

After Surgery programme for colonic surgery

Freek Gillissen, Stephanie M C Ament, José M C Maessen, Cees H C Dejong,

Carmen D Dirksen, Trudy van der Weijden, Maarten F von Meyenfeldt

Published: World Journal of surgery 2015 Feb;39(2):526-33 


\section{CHAPTER 4}

\section{Abstract}

\section{Background}

Between 2006 and 2009 the enhanced recovery after surgery (ERAS) programme for colonic surgery was implemented in one-third of all hospitals in the Netherlands ( $\mathrm{n}=$ 33). This resulted in enhanced recovery and a decrease in hospital length of stay (LOS) from a median of 9 days at baseline to six days at one-year follow-up. The present study assessed the sustainability of the ERAS programme three to five years after its implementation.

\section{Materials and methods}

From the 33 ERAS hospitals, ten initially successful hospitals were selected, with success defined as a median LOS of six days or lower and protocol adherence rates above $70 \%$. In 2012 a retrospective audit of 30 consecutive patients was performed in each of these hospitals. Sustainability of the ERAS programme was assessed on hospital level, using median hospital LOS, protocol adherence rates and time needed for functional recovery. Data were compared with the implementation phase data.

\section{Results}

Overall median LOS in the selected hospitals increased from 5.25 days (interquartile range [IQR] 4.75-6.00; min: 4.00 - max: 6.00) to 6 days (IQR 5.00-7.00; min: 5.00 - max: $8.00)$, but this change was not significant $(p=0.052)$. Time to functional recovery was equal in both phases: median 3.00 days $(p=0.26)$. Protocol adherence decreased from 75 to $67 \%(p=0.32)$. Especially adherence to postoperative care elements dropped considerably.

\section{Conclusions}

Despite a slight decrease in protocol adherence, the ERAS programme was sustained reasonably well in the ten selected hospitals, although there was quite some variation between the hospitals. 
SUSTAINABILITY OF ENHANCED RECOVERY AFTER SURGERY (ERAS)

\section{Introduction}

Many innovations have been implemented in the last decades, some more successful than others $[1,2]$. Implementing an effective innovation into daily routines is, generally speaking, a dynamic and difficult process [3], and results may vary between organisations [4-6]. Furthermore, once implementation has succeeded there often is a tendency for relapse into old routines after the implementation activities have ended [7]. Research shows that in public health $40 \%$ of all innovations are not sustained after termination of the initial implementation activities and funding [8]. This may be seen as a waste of time and money spent on the implementation. Besides the financial implications, the discontinuation of successful innovations might result in less than optimal care for patients, and it may cause frustration and diminish the support for future health care initiatives. Therefore, it is important that achieved benefits of an effective intervention are sustained after implementation.

Although sustainability is an important subject in health care, there is no standard definition of sustainability [9]. It can be seen as "holding the gains" or "maintaining health benefits" and "continuation of the programme activities within an organisational structure" [7, 9-11]. Sustainability of change exists when a newly implemented innovation continues to deliver the achieved benefits over a longer period of time, certainly does not return to the usual processes and becomes "the way things are done around here," even after the implementation project is no longer actively carried out or until a better innovation comes along [12]. Furthermore, little is known about differences in sustainability success between hospitals, although a recent study suggested that insight into practice variation might improve the quality of care [13]. Surgical research tends to focus on overall results on the patient level, but because it has been suggested that organisations differ in their innovative climate and urge to implement new insights, it is likely that changes are sustained in varying degrees in different hospitals.

Surgery, as other specialties, is a domain in health care that has a high turn-around and a fast-changing character, with many innovations in technology [14]. Many innovations have been implemented in recent decades, such as minimally invasive surgery, endovascular surgery, and ancillary innovations aimed at improving quality of care [1517]. Another example is the introduction of evidence-based multimodal perioperative care protocols, leading to an enhanced recovery and a reduction in hospital length of stay (LOS) in surgical patients [18-22]. In the Netherlands, the enhanced recovery after surgery (ERAS) programme was implemented in colonic surgery in 33 hospitals by means of a generic implementation strategy, the so-called Breakthrough Series. This resulted in enhanced recovery and an overall decrease in LOS after colonic surgery, from a median of nine days at baseline to six days after one year, at the end of the implementation strategy [23]. To study whether these results were sustained we assessed the benefits achieved of the perioperative care programme three to five years after the implementation strategy had been ended. 


\section{CHAPTER 4}

\section{Methods}

For the long-term follow-up measurement we used a retrospective observational study design. As part of the primary implementation project (the Breakthrough Study) the participating hospitals had already performed a retrospective baseline measurement of surgical care during the year before implementation (2005-2007; pre-implementation phase), and a prospective measurement of care performed during the implementation project (2006-2009; implementation phase). This Breakthrough Study ended in 2009. During the present study a retrospective analysis of surgical care was performed (2012; post-implementation phase). This long-term evaluation study was not planned as part of the initial Breakthrough Study; as such, it is independent of the original implementation study. To assess sustainability, results of the post-implementation phase were compared with the implementation phase. Results of the pre-implementation phase were presented for further information.

\section{Ethical approval and informed consent}

The Medical Ethical Committee of the University of Maastricht granted approval for the project, METC 11-4-015.10. The privacy of the included patients was protected, and all data were coded and processed anonymously. Medical files with explicit patient statements that their medical information should not be used for clinical research were not included.

\section{Participants}

\section{Hospitals}

Ten hospitals were selected out of the total of 33 hospitals that had participated in the original Breakthrough Study. Only those hospitals were selected in which the primary implementation strategy was successfully executed. Initial implementation success was defined as follows: (1) a median LOS of 6 days or lower, (2) an overall protocol adherence above $70 \%$, and (3) at least 40 patients treated within the year of the implementation project.

\section{Patients}

For the post-implementation audit of each selected hospital, 30 consecutive patients undergoing colorectal surgery were included from the period 2011-February 2012. The same inclusion and exclusion criteria were used as in the primary implementation project: patients undergoing elective colorectal resection above the peritoneal reflection for benign or malignant disease were eligible for this study. Patients who needed emergency surgery and those requiring an end or diverting ileostomy or colostomy were excluded. 


\section{Outcomes}

Sustainability was assessed according to three different outcomes: LOS, functional recovery, and protocol adherence rates. These results were compared with the results of the audit during implementation. We defined sustainability at the hospital level as being reached if the performance in the post-implementation phase was equal to or better than performance during the implementation phase.

\section{Length of stay}

Hospital length of stay was defined as the number of nights in hospital after surgery.

\section{Time to functional recovery}

Time to functional recovery (FR) was defined as the number of postoperative days until adequate pain control requiring oral analgesia only was achieved, as well as tolerance of solid foods and independent mobility sufficient to perform activities of daily living at the preoperative level.

\section{Protocol adherence}

The performance on key elements of the ERAS programme was evaluated per element.

\section{Data collection}

Data collection started in March 2012. Hospitals were asked to provide a list of the last consecutive 30 patients who underwent elective colonic surgery, until the start of data collection. Data were extracted from the (electronic) patient files by two of the authors ( $F G, S A)$. In the beginning this was done as a joint exercise to guarantee standardisation of auditing methods. At a later stage, the data collection was done by one of these two authors separately.

\section{Statistical analysis}

Differences between the pre-implementation, implementation, and post-implementation phases were checked using the Mann-Whitney $U$ test for continuous variables and the Chi-square test for categorical variables. Differences in LOS and FR between the implementation phase and the post-implementation phase were analysed with log-rank tests, as LOS and FR were censored if the patient had died. A p value of $\leq 0.05$ was considered statistically significant. The unit of analysis was hospital level, to assess the variation between hospitals. Continuous variables are presented as means and standard deviations (SD) or as median and range in the case of skewed data. Both LOS and FR are presented as median of the median per hospital. Interquartile ranges and minimum and maximum values are presented between bars. Number and percentage were used for categorical variables. All analyses were performed with SPSS 20.0 (SPSS Inc., Chicago, IL). In the pre-implementation phase, fewer data were available for analysis. These are reported as not available/missing (NA) in the Results section. 


\section{CHAPTER 4}

\section{Results}

The ten selected hospitals participating in the project included seven teaching hospitals and three non-teaching hospitals. In the pre-implementation phase 450 patients in total were included. In the implementation phase 523 patients were included, with 297 patients remaining in the post-implementation phase. Overall demographics are shown in table 1 . In the post-implementation phase significantly older patients were included, with more patients in American Society of Anesthesiologists (ASA) grade III/IV and more patients with a malignancy $(p<0.001)$. Also, in the post-implementation phase, more than half of the surgical procedures were performed laparoscopically, whereas some 40 $\%$ of procedures were laparoscopic during the implementation phase $(p=0.45)$. Results on LOS, FR, and protocol adherence are shown on hospital level (table 2).

Table 1 Comparison of overall patient characteristics of the pre-implementation and implementation with the post-implementation phase on the hospital level ${ }^{\mathrm{a}}$

\begin{tabular}{lllll}
\hline & $\begin{array}{l}\text { Pre-implementation } \\
\mathrm{n}=450\end{array}$ & $\begin{array}{l}\text { Implementation } \\
\mathrm{n}=532\end{array}$ & $\begin{array}{l}\text { Post-implementation } \\
\mathrm{n}=297\end{array}$ & P Value \\
\hline Sex Male (\%) & 50 (SD 0.5, R 40-61) & 49 (SD 0.5 R 41-56) & 48 (SD 0.5 R 39-63) & 0.75 \\
Mean age & 64 (SD 14.5 R 60-69) & 66 (SD 13.4 R 61-70) & 70 (SD 11.1 R 67-73) & 0.001 \\
ASA III/IV (\%) & NA & 12 (SD0.7 R 0-42) & 20 (SD 0.8 R 3-41) & 0.001 \\
Malignancy (\%) & NA & 63 (SD 0.9 R 41-86) & 84 (SD 0.9 R 57-100) & 0.001 \\
Laparoscopy(\%) & NA & 43 (SD 0.5 R 0-83) & 56 (SD 0.6 R 21-90) & 0.45 \\
\hline
\end{tabular}

${ }^{a}$ Numbers between parentheses are the standard deviation (SD) and the range between hospitals (R) (excluding missing values)

ASA: American Society of Anesthesiologists, NA: Not Available

Table 2 Overall results of the implementation and the post-implementation phase per hospital ${ }^{\mathrm{a} * \mathrm{~B}^{*}>}$

\begin{tabular}{|c|c|c|c|c|c|c|}
\hline & \multicolumn{2}{|l|}{ LOS } & \multicolumn{2}{|l|}{ FR } & \multicolumn{2}{|c|}{ Adherence } \\
\hline & Impl & Post-impl & $\mid \mathrm{mpl}$ & Post-impl & Impl & Post-impl \\
\hline Hospital 1 & 6 & 7 & 3 & 3 & 77 & 61 \\
\hline Hospital 2 & 4 & 8 & 3 & 4 & 82 & 70 \\
\hline Hospital 3 & 5 & 5 & 3 & 2 & 71 & 56 \\
\hline Hospital 4 & 5 & 6 & NA & 3 & 65 & 68 \\
\hline Hospital 5 & 6 & 6.5 & 3 & 3 & 78 & 60 \\
\hline Hospital 6 & 4 & 6 & 3 & 3 & 87 & 69 \\
\hline Hospital 7 & 5 & 7.5 & 3 & 3 & 80 & 73 \\
\hline Hospital 8 & 5.5 & 6 & 3 & 2.5 & 75 & 68 \\
\hline Hospital 9 & 6 & 5 & 4 & 3 & 68 & 70 \\
\hline Hospital 10 & 6 & 5 & 4 & 3 & 64 & 72 \\
\hline
\end{tabular}

${ }^{a}$ Data of length of stay (LOS) and functional recovery (FR) are median days. Data regarding adherence are percentages. NA: Not Available, Impl: implementation 


\section{Length of stay}

Overall, the median LOS increased from 5.25 days (IQR P25 4.75- P75 6.00; min 4.00; $\max 6.00$ ) in the implementation phase to 6.00 days (IQR P25 5.00-P75 7.00; min 5.00; $\max 8.00$ ) in the post-implementation phase, but this difference was not significant ( $p=$ 0.052). Hospital LOS was sustained in three hospitals: two hospitals achieved a further reduction in LOS in the post-implementation phase, and one hospital showed an equal LOS. Seven hospitals showed an increase in LOS in the post-implementation phase. One of these hospitals fell back to the same level noted before the implementation phase (figure 1).

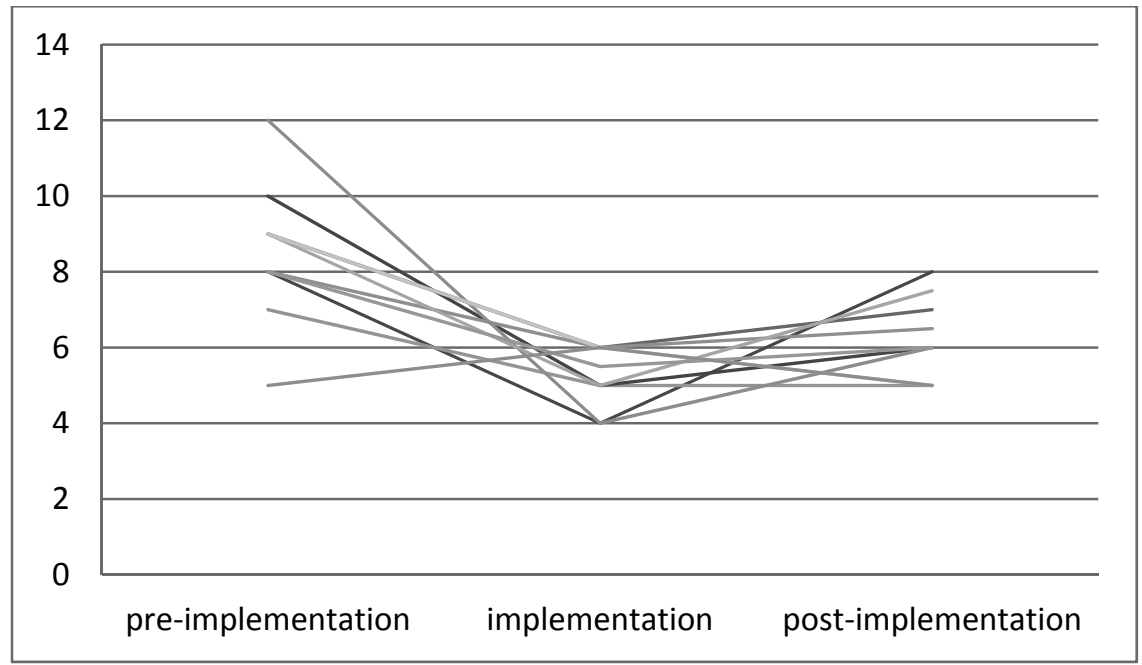

Figure 1. Median length of stay per hospital in the pre-implementation, implementation and postimplementation groups of the Enhanced Recovery after Surgery (ERAS) programme ${ }^{a}$

${ }^{a}$ Two hospitals reached the same result; those findings are shown as one line

\section{Functional recovery}

Overall FR was reached in a period of median 3.00 days (IQR P25 2.88-P75 3.00; min 2.00; $\max 4.00)$ in the post-implementation phase, which was equal to the median FR 3.00 (IQR P25 3.00-P75 3.50; $\min 3.00 ; \max 4.00$ ) in the implementation phase. Time to FR was sustained in eight hospitals: three hospitals showed a further reduction of FR of one day, and time to FR remained equal in five hospitals. One hospital showed an increase in time to FR of one-and-a-half day. In one hospital no data were available for FR in the implementation phase. Overall, the proportion of patients recovered on postoperative day two increased from 18 to $37 \%$ (range 27-52\%), and increased on postoperative day three from 54 to 64\% (range 40-85\%). These results differed markedly between hospitals, the proportion of patients showing FR on day two in the bestperforming hospital being $52 \%$, while FR was $27 \%$ in the hospital with the poorest performance. The same observation was made on the third postoperative day: in the best- 


\section{CHAPTER 4}

performing hospital $85 \%$ of patients were functionally recovered on the third postoperative day, whereas in the least-performing hospital $40 \%$ of patients were functionally recovered on this day (figure 2 ).
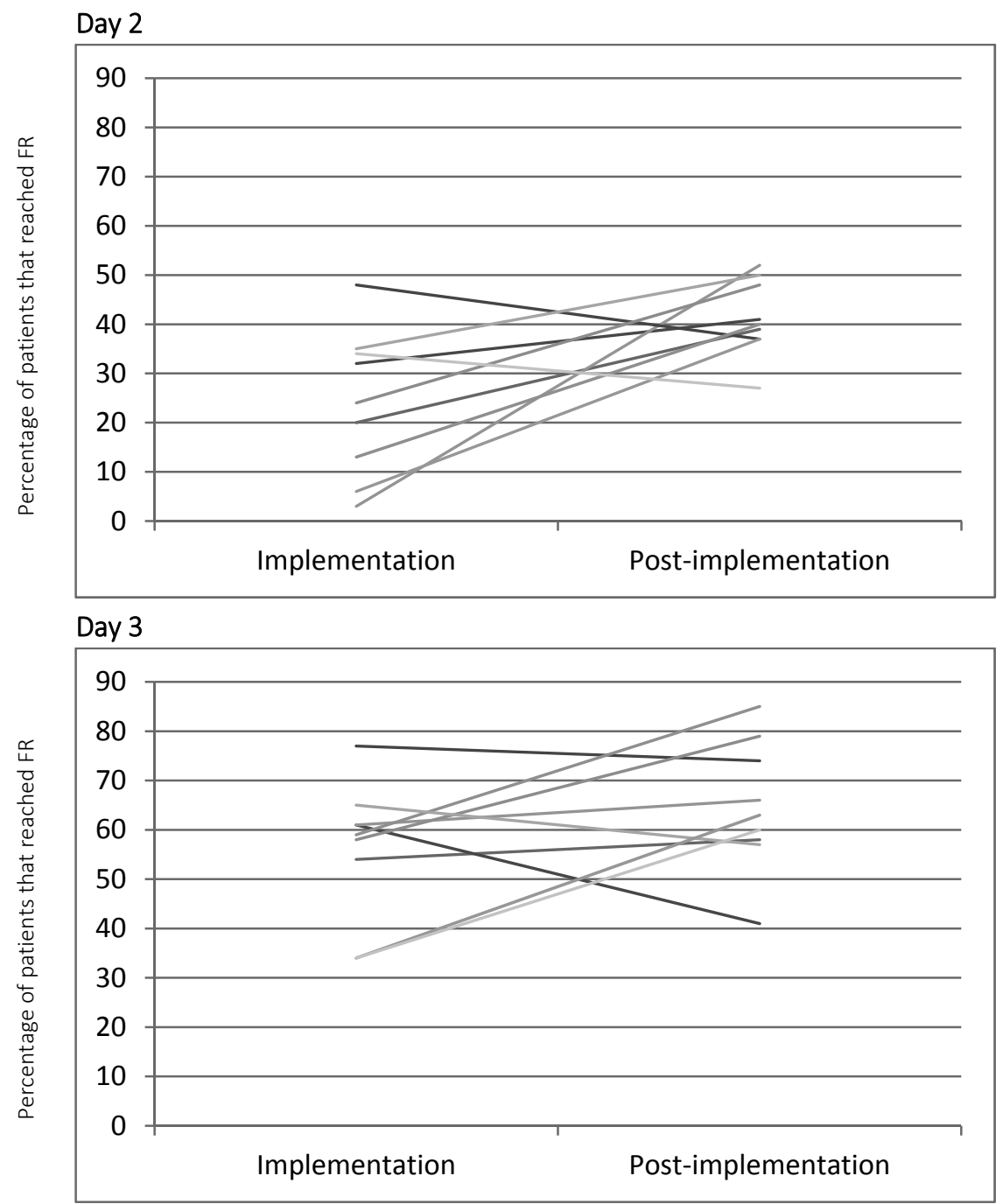

Figure 2 Cumulative percentages of proportion of patients functionally recovered (FR) per hospital on postoperative day 2 and day 3 


\section{Protocol adherence}

Overall mean protocol adherence rate in the post-implementation phase was $67 \%$ (SD $6.3 \mathrm{~min} 56 \%$; $\max 73 \%$ ), whereas it was 75\% (SD $7.6 \mathrm{~min} 64 \%$; $\max 87 \%$ ) in the implementation phase. Overall adherence in the preoperative and perioperative period remained almost equal in the implementation and post-implementation phases, but postoperative adherence dropped considerably in the post-implementation phase (table 3) Particularly, the cessation of IV fluids on the first postoperative day (from 36 to 9\%), mobilisation for more than three hours (90-38\%), and resumption of solid foods (65$37 \%)$ were protocol elements less adhered to. There was a wide variation between hospitals. Three hospitals sustained their performance on protocol adherence, two hospitals showed a reduction of less than $10 \%$, and five hospitals showed a reduction of adherence of more than $10 \%$ (figure 3).

Table 3 Protocol adherence in the implementation and post-implementation phase

\begin{tabular}{|c|c|c|c|}
\hline Perioperative care elements & $\begin{array}{l}\text { Implementation } \\
\mathrm{n}=523\end{array}$ & $\begin{array}{l}\text { Post-implementation } \\
\mathrm{n}=297\end{array}$ & P Value \\
\hline \multicolumn{4}{|l|}{ Before surgery } \\
\hline Pre-admission counselling & 78 (SD 20.5 R 33-100) & 87 (SD 22.5 R 22-100) & $<0.01$ \\
\hline No bowel preparation & 98 (SD 3.5 R 88-100) & 87 (SD 19.8 R 40-100) & $<0.01$ \\
\hline Oral carbohydrate treatment & 88 (SD 11.4 R 58-98) & 86 (SD 22.8 R 22-100) & $<0.01$ \\
\hline Preoperative adherence & 90 (SD 8.2 R 76-99) & 87 (SD 10.6 R 68-99) & $<0.01$ \\
\hline \multicolumn{4}{|l|}{ During surgery } \\
\hline $\begin{array}{l}\text { Active warming by upper body air- } \\
\text { warming device }\end{array}$ & 100 (SD 0.6 R 98-100) & 99 (SD 2.3 R 93-100) & 0.17 \\
\hline Epidural anesthesia & 90 (SD 11.9 R 59-100) & 81 (SD 23.7 R 29-97) & $<0.01$ \\
\hline $\begin{array}{l}\text { Nasogastric drainage removed at } \\
\text { end of surgery }\end{array}$ & 93 (SD 8.4 R 71-100) & 97 (SD 3.3 R 93-100) & 0.02 \\
\hline Perioperative adherence & 95 (SD 5.3 R 85-100) & 92 (SD 9.1 R 74-99) & $<0.01$ \\
\hline \multicolumn{4}{|l|}{ Postoperative elements, day 0} \\
\hline Mobilisation > 15 minutes & 69 (SD 22.8 R 20-100) & 50 (SD 19.6 R 9-87) & $<0.01$ \\
\hline Oral fluids intake $>500 \mathrm{ml}$ & 67 (SD 24.9 R 0-93) & 65 (SD 17.5 R 20-80) & $<0.01$ \\
\hline \multicolumn{4}{|l|}{ Day 1 after surgery } \\
\hline IV fluid infusion stopped & 36 (SD 25.6 R 3-87) & 9 (SD 6.0 R 0-17) & $<0.01$ \\
\hline Mobilisation $>3$ hours & 90 (SD 21.8 R 83-100) & 38 (SD 14.7 R 17-66) & $<0.01$ \\
\hline Oral nutritional supplements & 63 (SD 36.1 R 0-98) & 70 (SD 28.6 R 3-100) & $<0.01$ \\
\hline Oral laxatives (MgO) & 78 (SD 6.3 R 77-100) & 94 (SD 7.4 R 78-100) & $<0.01$ \\
\hline \multicolumn{4}{|l|}{ Day 2 after surgery } \\
\hline Epidural removed & 72 (SD 21.9 R 15-98) & 79 (SD 16.0 R 50-93) & $<0.01$ \\
\hline Postoperative adherence & 70 (SD 15.2 R 47-94) & 58 (SD 7.2 R 44-71) & $<0.01$ \\
\hline Overall compliance rate & 75 \% (SD 7.6 R 64-87) & 67 \% (SD 6.3 R 56-73) & $<0.01$ \\
\hline
\end{tabular}

${ }^{a}$ Presented are the percentages (standard deviation SD and the range between hospitals $\mathrm{R}$ ) of patients treated according to the ERAS programme elements, overall, and for the preoperative, perioperative and postoperative elements separately 


\section{CHAPTER 4}

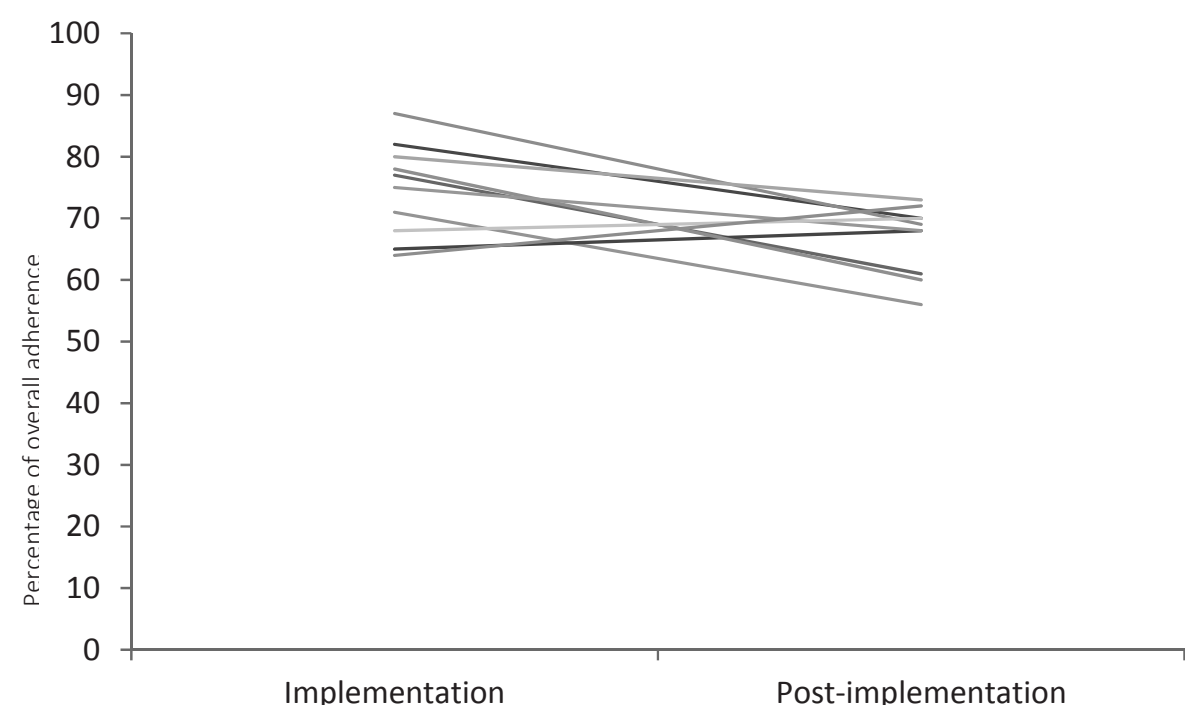

Figure 3 Adherence to the protocol rate per hospital in the implementation and the post-implementation phase

\section{Discussion}

The present study shows that initial results of a large-scale implementation project of the ERAS programme were sustained reasonably well, although there was a large variation among hospitals. The primary aim of the evidence-based perioperative care programme was to decrease the time to postoperative recovery and, subsequently, the time of hospitalisation. The present long-term follow-up demonstrated that time to recovery was sustained in most hospitals, whereas the achieved reduction in LOS was not fully sustained in all hospitals. The implementation successes in protocol adherence were not very well sustained, as, in most hospitals, adherence rates dropped in the post-implementation phase.

With regard to LOS, other studies showed a median of two days after colonic surgery within a fast track protocol $[20,24]$, but these results were not reached in the present study. In the Netherlands, the overall median LOS after colonic surgery was 7-8 days in 2012 [25]. Although the optimum was not reached, nine of the ten hospitals in the current study performed considerably better with respect to LOS than the rest of the Netherlands, and LOS of only one hospital increased to eight days. These results suggest that the ERAS programme is still effective in the participating hospitals. The fact that a LOS of two days after colonic surgery was not reached could be due to the health care policy regarding reimbursement in the Netherlands. The system does not stimulate discharge of patients as soon as they are capable of going home (FR). Evaluation of the 
initial implementation results showed that all hospitals had difficulties with discharging patients at the moment of FR [23]. The present study shows that the gap between FR and discharge of postoperative patients remains large. More research is needed to determine possible reasons for this gap that prohibits reaching a hospital LOS of two days, as seen in other countries.

Despite a significant increase in the age of the patients and a larger proportion of ASA III/IV patients who were operated and cared for according to the ERAS programme, LOS and FR were sustained in several hospitals. This might be explained by the proportion of patients operated laparoscopically being significantly larger in the postimplementation phase compared with the implementation phase, again with a wide variation among hospitals (range 21-90\%).

In contrast to the preoperative and perioperative adherence rates of the ERAS programme, the adherence rates to postoperative care elements in our series dropped remarkably, similar to other studies [26]. In particular, adherence to three protocol elements deteriorated: (1) mobilisation of patients for more than three hours on the first postoperative day, (2) cessation of IV fluids on the first postoperative day, and (3) resumption of solid foods. These postoperative protocol elements were also those that were the most difficult to adhere to in the implementation phase [23]. Postoperative mobilisation of patients and the cessation of IV fluids are elements in postoperative care that are influenced by multiple factors, such as patient characteristics, the doctors on the ward, the nurses, and physiotherapists. The number of professionals involved may be causal for the difficulty to sustain the required changes, particularly if there is no specific attention to the continuation of these elements. Interestingly, the best performing hospital with regard to length of stay and recovery showed a low protocol adherence rate, whereas the least performing hospital showed a high adherence rate. It therefore remains unclear what elements of the ERAS programme are the determinants of success of implementation.

Hospitals in the present study were selected because they initially showed good results on LOS and adherence rates. This suggests that implementation of the ERAS programme caused a change in the mind-set of health care professionals in these hospitals [27, 28], although this kind of change has been suggested to be difficult $[1,29,30]$. After the initial implementation, the ERAS programme needs to be normalised within the local hospital organisation. Because results on sustainability of the ERAS programme varied remarkably, it could be argued that this change in mind-set was not equally strong in every hospital.

A strength of the study is that we analysed surgical care three to five years after the primary implementation of the ERAS programme. The median follow-up time for interventions aimed at improving quality of care is generally less than one year [31], although a recent review shows that more than $50 \%$ of sustainability studies are performed two years or later after implementation [32]. By expanding the follow-up peri- 
od, more insight is gained regarding the long-term impact of implementing change and its sustainability. If an innovation is effective the first year after implementation but the effect dies out in the succeeding years, the benefit of the innovation becomes questionable.

Another strength of the study is the analysis on the hospital level. Most surgical implementation studies present results on the patient level, overlooking institutional variation [6]. By analysing results on the hospital level, more detailed insight is gained into the variation in performance of the ERAS programme in each hospital. The choice to analyse the data on hospital level, however, made it impossible to develop a reliable multivariate regression model. This means that the influence on LOS of important factors such as laparoscopy and rising patient age was not assessed.

A further strong point of the present study is the definition of sustainability. We applied a narrative definition based on the literature, and in light of this definition we specified endpoints applicable for this specific case, the ERAS programme for colonic surgery. Choosing specific endpoints is necessary for a transparent and meaningful evaluation of sustainability. We realise, however, that our endpoints cannot be applied universally. Another issue concerning these endpoints was the use of three outcome parameters. Because all three are influenced by several factors other than the patient alone, it was impossible to conclude which outcome parameter is the most important one. Therefore, we did not provide an overall result regarding sustainability, but assessed sustainability according to these three parameters separately.

A weakness of the current study is the retrospective character of the audit. A problem with auditing patient files retrospectively could be that not all process indicators were recorded accurately, meaning that the actual performance on care elements could have been underestimated by a lack of registration. However, by choosing a retrospective audit, a Hawthorne effect was prevented. This is the phenomenon that a team of professionals is improving its performance due to awareness of being evaluated. Thus, although performance on some process indicators may have been underestimated, the retrospective audit provides a more honest insight into the actual care provided.

Another weakness of the study is the restriction to a subsample of hospitals. It was not possible to assess the sustainability of the ERAS programme in all 33 hospitals because of the limitations of time and resources. We therefore chose the best-performing hospitals at the time of primary implementation of the ERAS programme, as successful primary implementation is a prerequisite for a sustainability evaluation in the longterm. We assume that the overall sustainability of all hospitals in the ERAS group is lower than the study group of the ten best-performing hospitals, although it is theoretically possible that the other 23 hospitals kept developing colonic surgery after the implementation phase, and showed a delayed success with reduction in median LOS and FR because of a slow learning curve. This could mean that these results might not be generalisable to all hospitals. 
SUSTAINABILITY OF ENHANCED RECOVERY AFTER SURGERY (ERAS)

\section{Conclusions}

This study shows that the ERAS programme was reasonably well sustained, although results varied remarkably between hospitals. Six years after the implementation of the ERAS programme median LOS increased from 5.25 to 6 days, but this result was not statistically significant. There was, however, a large variation among hospitals regarding LOS, time to FR, as well as protocol adherence. 


\section{References}

1. Grol R, Wensing M. What drives change? Barriers to and incentives for achieving evidence-based practice. Med J Aust. 2004 Mar 15;180(6 Suppl):S57-60.

2. Wilson KD, Kurz RS. Bridging implementation and institutionalization within organizations: proposed employment of continuous quality improvement to further dissemination. J Public Health Manag Pract. 2008 Mar-Apr;14(2):109-16.

3. Grol R, Grimshaw J. From best evidence to best practice: effective implementation of change in patients' care. Lancet. 2003 Oct 11;362(9391):1225-30.

4. Kitson A, Harvey G, McCormack B. Enabling the implementation of evidence based practice: a conceptual framework. Quality in health care : QHC. 1998 Sep;7(3):149-58.

5. Scott-Findlay S, Golden-Biddle K. Understanding how organizational culture shapes research use. The Journal of nursing administration. 2005 Jul-Aug;35(7-8):359-65.

6. Rangachari P, Rissing P, Rethemeyer K. Awareness of evidence-based practices alone does not translate to implementation: insights from implementation research. Quality management in health care. $2013 \mathrm{Apr}-$ Jun;22(2):117-25.

7. Scheirer MA. Is Sustainability Possible? A Review and Commentary on Empirical Studies of Program Sustainability. American Journal of Evaluation. 2005 September 2005;26(3):27.

8. Schell SF, Luke DA, Schooley MW, Elliott MB, Herbers SH, Mueller NB, et al. Public health program capacity for sustainability: a new framework. Implementation science : IS. 2013;8:15

9. Shediac-Rizkallah MC, Bone LR. Planning for the sustainability of community-based health programs: conceptual frameworks and future directions for research, practice and policy. Health Educ Res. 1998 Mar;13(1):87-108.

10. Gruen RL, Elliott JH, Nolan ML, Lawton PD, Parkhill A, McLaren CJ, et al. Sustainability science: an integrated approach for health-programme planning. Lancet. 2008 Nov 1;372(9649):1579-89.

11. Rabin BA, Brownson RC, Haire-Joshu D, Kreuter MW, Weaver NL. A glossary for dissemination and implementation research in health. J Public Health Manag Pract. 2008 Mar-Apr;14(2):117-23.

12. Ament SMC, Gillissen F, Maessen JMC, Dirksen CD, van der Weijden T, von Meyenfeldt MF. Sustainability of healthcare innovations (SUSHI): long term effects of two implemented surgical care programmes (protocol). BMC health services research. 2012;12:423. PubMed PMID: 23174024.

13. Tomson $C R$, van der Veer SN. Learning from practice variation to improve the quality of care. Clinical medicine (London, England). 2013 Feb;13(1):19-23.

14. Rogers WA, Lotz M, Hutchison K, Pourmoslemi A, Eyers A. Identifying Surgical Innovation: A Qualitative Study of Surgeons' Views. Ann Surg. 2013 Jun 19.

15. Cahill RA, Lindsey I. Operative innovations for colorectal cancer. Colorectal Dis. 2011 Nov;13 Suppl 7:1-2.

16. Mathis KL, Boostrom SY, Pemberton JH. New developments in colorectal surgery. Curr Opin Gastroenterol. $2013 \operatorname{Jan} ; 29(1): 72-8$.

17. Atallah S, Nassif G, Polavarapu H, Debeche-Adams T, Ouyang J, Albert M, et al. Robotic-assisted transanal surgery for total mesorectal excision (RATS-TME): a description of a novel surgical approach with video demonstration. Tech Coloproctol. 2013 Jun 26.

18. Fearon KC, Ljungqvist O, Von Meyenfeldt M, Revhaug A, Dejong CH, Lassen K, et al. Enhanced recovery after surgery: a consensus review of clinical care for patients undergoing colonic resection. Clin Nutr. 2005 Jun;24(3):466-77.

19. Kehlet $\mathrm{H}$. Multimodal approach to control postoperative pathophysiology and rehabilitation. Br J Anaesth. 1997 May; 78(5):606-17.

20. Kehlet $\mathrm{H}$, Mogensen $\mathrm{T}$. Hospital stay of 2 days after open sigmoidectomy with a multimodal rehabilitation programme. Br J Surg. 1999 Feb;86(2):227-30.

21. Varadhan KK, Neal KR, Dejong CH, Fearon KC, Ljungqvist O, Lobo DN. The enhanced recovery after surgery (ERAS) pathway for patients undergoing major elective open colorectal surgery: a meta-analysis of randomized controlled trials. Clin Nutr. 2010 Aug;29(4):434-40. 


\section{SUSTAINABILITY OF ENHANCED RECOVERY AFTER SURGERY (ERAS)}

22. Vlug MS, Wind J, Hollmann MW, Ubbink DT, Cense HA, Engel AF, et al. Laparoscopy in Combination with Fast Track Multimodal Management is the Best Perioperative Strategy in Patients Undergoing Colonic Surgery: A Randomized Clinical Trial (LAFA-study). Ann Surg. 2011 May 18.

23. Gillissen F, Hoff C, Maessen JMC, Winkens B, Teeuwen JH, von Meyenfeldt MF, et al. Structured synchronous implementation of an enhanced recovery program in elective colonic surgery in 33 hospitals in The Netherlands. World journal of surgery. 2013 May;37(5):1082-93.

24. Rossi G, Vaccarezza H, Vaccaro CA, Mentz RE, Im V, Alvarez A, et al. Two-day Hospital Stay After Laparoscopic Colorectal Surgery under an Enhanced Recovery after Surgery (ERAS) Pathway. World J Surg. 2013 Jul 24.

25. DSCA. Jaarrapportage 2012 Dutch Surgical Colorectal Audit Leiden: DICA; 2012.

26. Hendry PO, Hausel J, Nygren J, Lassen K, Dejong CH, Ljungqvist O, et al. Determinants of outcome after colorectal resection within an enhanced recovery programme. Br J Surg. 2009 Feb;96(2):197-205.

27. Biffl WL, Spain DA, Reitsma AM, Minter RM, Upperman J, Wilson M, et al. Responsible development and application of surgical innovations: a position statement of the Society of University Surgeons. Journal of the American College of Surgeons. 2008 Jun;206(3):1204-9.

28. Ahmed J, Khan S, Lim M, Chandrasekaran TV, MacFie J. Enhanced recovery after surgery protocols - compliance and variations in practice during routine colorectal surgery. Colorectal Dis. 2012 Sep;14(9):104551.

29. Grimshaw JM, Eccles MP, Walker AE, Thomas RE. Changing physicians' behavior: what works and thoughts on getting more things to work. J Contin Educ Health Prof. 2002 Fall;22(4):237-43.

30. Polle SW, Wind J, Fuhring JW, Hofland J, Gouma DJ, Bemelman WA. Implementation of a fast-track perioperative care program: what are the difficulties? Dig Surg. 2007;24(6):441-9.

31. Hovlid E, Bukve O, Haug K, Aslaksen AB, von Plessen C. Sustainability of healthcare improvement: what can we learn from learning theory? BMC health services research. 2012;12:235.

32. Wiltsey Stirman S, Kimberly J, Cook N, Calloway A, Castro F, Charns M. The sustainability of new programs and innovations: a review of the empirical literature and recommendations for future research. Implementation science : IS. 2012;7:17. 



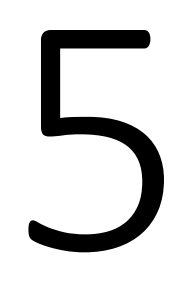

\section{Analysing sustainability of economic value using the net monetary benefit approach: The case of a short stay programme for breast cancer surgery care five years after implementation}




\section{CHAPTER 5}

\section{Abstract}

\section{Objectives}

The aim of this study was to perform an analysis of sustainability of economic value using the net monetary benefit (NMB) approach. The application of the approach was demonstrated by using an exemplary implementation case on a short stay programme for breast cancer surgery (SSP).

\section{Methods}

Sustainability of economic value was determined in terms of the incremental net mone-

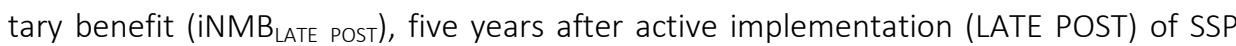
compared to early post-implementation results (POST). According to the NMB approach, the economic value is fully sustained if the iNMB $B_{\text {LATE POST }}$ equals or is greater than zero. If the iNMB ${ }_{\text {LATE POST }}$ is negative, this represents the value of sustainability.

\section{Results}

Using a lambda of $€ 80.000$, the economic value of the SSP was fully sustained with a mean pooled iNMB $_{\text {LATE POST }}$ of $€ 1649$ per patient. After adjustment for age, iNMB

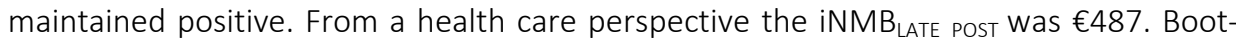
strap analyses showed that the probability that the economic value of SSP was sustained was $95 \%$ and $65 \%$ respectively from a societal and a health care perspective (lambda: €0-€75.000).

\section{Conclusions}

Results showed that sustainability of economic value of health care innovations can be calculated using the NMB approach. Application of the NMB approach for the analysis of sustainability of economic value raised some issues, such as whether regression analysis should be performed to correct for differences in case-mix over time, the appropriate perspective, the level of analysis (macro or meso level), and the appropriate timing of analysis. 


\section{Introduction}

In budget-constrained health care systems it is key to invest in valuable and sustainable innovations. To this end, the concept of sustainability is increasingly used in the health care domain. For example, sustainability is used in terms of creating a health care system in which health care maintains affordable and of high quality [1]. Following Berwick, a sustainable health care system can be created if the Triple Aim [2], i.e. improving the populations' health, delivering better health care as experienced by the patient and increasing the value for money of health care, is being integrated in policies.

In relation to implementation of health care innovations another focus of the concept of sustainability in health care can be used [3]. In this context, an innovation is sustained if, following implementation, it continues to deliver the benefits achieved over a longer period of time, the people involved do not return to the usual processes [4] and the innovation becomes 'the way things are done around here' [5], even after the implementation project is no longer actively carried out, until a better innovation comes along [6]. The sustainability of innovations is essential in order to optimise health care delivery continuously. Moreover, sustaining evidence-based innovations in daily clinical practice ultimately adds to sustainability of the health care system from a macro-system perspective. Nevertheless, many innovations which are initially successfully introduced in practice, fail to become fully sustained in routine care $[7,8]$.

Sustainability of evidence-based innovations in practice is a relatively new concept in health care and various definitions are used $[7,9,10]$. A recent review showed that quantitative sustainability evaluation studies in health care mainly focus on maintaining the innovations' benefits in terms of health care professionals' adherence and clinical outcomes [7]. However, according to Porter [11], value instead of intermediate outcomes at patient level and professionals' adherence to the innovation should be the primary focus when improving health care, where value represents the favourable balance between the input (costs) and the output (health outcomes) of health care innovations. Porter stated that "if value improves, patients, payers, providers, and suppliers can all benefit while the economic sustainability of the health care system increases." Not sustaining innovations implies a waste of the efforts and money invested in implementation activities. Furthermore, sustainability of innovations in terms of, for example, health outcomes and adherence to programme elements, does not automatically imply sustainability of economic value. Economic value may be not sustained, due to for example political, demographic or socio-economic changes, or the emergence of new technologies.

Recently, Fenwick [12] introduced the concept of value of implementation which uses a net monetary benefit (NMB) approach. Value of implementation represents the opportunity loss as a consequence of suboptimal health care delivery [13]. The aim of this study is to investigate how the NMB approach for the analysis of sustainability of economic value in health care after implementation of an innovation can be applied. 


\section{CHAPTER 5}

First, the exemplary case will be described, that is, implementation of a short stay programme for breast cancer surgery patients in four early adopter hospitals in the Netherlands. Then, the use of the NMB approach to analyse the sustainability of economic value will be described. Based on the analyses, this study will reflect on how the application of the NMB approach can be used to analyse the sustainability of economic value five years following implementation of the short stay programme.

\section{Methods}

\section{Illustrative case study}

\section{A short stay programme for breast cancer surgery care}

Between 2005 and 2007 a short stay programme was implemented in four early adopter hospitals, designed to enable short admission for patients undergoing breast cancer surgery. The short stay programme is a comprehensive programme which was developed to increase the efficiency of breast cancer surgery care, while maintaining the perceived quality of care by patients. Immediately after implementation (POST group, December 2006 - July 2007), societal costs and QALYs were measured with a time horizon of 6 weeks, as part of the primary implementation study [14]. Early postimplementation results showed successful results in terms of safety, quality and costeffectiveness. Mean societal costs decreased by €955 for patients who were treated after implementation of the comprehensive care programme, compared to traditional care, while quality of life in terms of Quality Adjusted Life Years (QALYs) and quality of care from a patient perspective were maintained [14]. As part of the SUSHI study, it was shown that the short stay programme was sustained in the four early adopter hospitals five years following implementation, in terms of proportion of patients treated in short stay and professionals' adherence to key recommendations [15]. This contribution focuses on the sustainability of economic value.

\section{Costs and effectiveness}

Societal costs and QALYs related to the full breast cancer surgery care process were remeasured in a prospective cohort of breast cancer patients in the same four hospitals five years following implementation (LATE POST group, June 2012 and June 2013). The same inclusion and exclusion criteria, data collection methods and analyses as in the primary implementation study were used to determine costs and effectiveness [14]. The study protocol of the SUSHI study has been published elsewhere (6) and the Medical Ethical Committee of the University of Maastricht granted approval, METC 11-4015.10. To compare the costs of the POST and LATE POST group, cost prices from the primary study were actualised from 2008 to 2012 and were used in this study. 
Data analysis

\section{Sustainability of economic value}

Sustainability of benefits achieved was assessed in terms of the continuation of economic value. Economic value comprises 'patient health outcomes achieved relative to the costs of care' [11]. Economic value was expressed in terms of the net monetary benefit (NMB). The NMB is a combined metric as it comprises both the monetary value of the health effect of care and the costs of care. Therefore, separate analyses on health effects (QALYS) and costs are not required when using the NMB approach. NMB has a linear nature and is reformulated from the ICER formulation as a function of lambda [16]. The NMB can be calculated by the formula: (QALYs $\times \lambda$ ) - costs (17).

The approach resembles the one used to determine the value of implementation [12] but is focused on the late post-implementation phase. Economic value was based on the costs and effects at two moments in time: POST (measurement in the early postimplementation phase, i.e. directly following implementation) and LATE POST (measurement in the late post-implementation phase, i.e. five years following implementation). To analyse the sustained level of the economic value achieved in the primary implementation study, the analysis focused on the NMB $\mathrm{NMATE}_{\text {POST }}$ and the NMB $\mathrm{B}_{\text {POST. }}$. Sustainability of economic value was determined by the incremental net monetary benefit

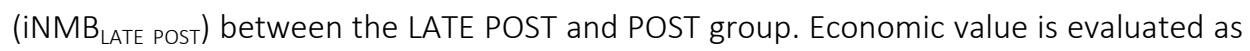
fully sustained if the iNMB $B_{\text {LATE POST }}$ equals or is greater than zero. An important assumption of the NMB approach is thus that the innovation is cost-effective and has been optimally implemented in the past [12]. Optimal implementation of an innovation reflects the perfect attainable implementation level in the real world, which may not be the same as the theoretical optimum of full (100\%) implementation. If the iNMB equals zero or is positive, no (additional) investment in sustainability activities is re-

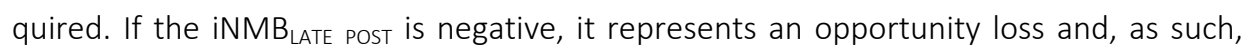
the value of sustainability. In the current study a $\lambda$ of $€ 80000$ was used for the base-case analysis.

Differences in patient characteristics were compared using the Mann-Whitney $U$ test for continuous variables and Chi-squared test for categorical variables between the POST and the LATE POST group. Multiple imputation was used to replace missing values of resource use and effectiveness data with plausible estimates. Cost, utility data and NMB results were pooled on imputation level. Bootstrapping was performed on base-

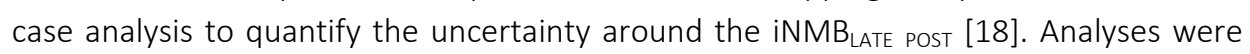
performed using SPSS 20.0 and Microsoft Excel 2010.

\section{Secondary analyses}

Several secondary analyses were performed in order to further explore the concept of sustainability of economic value. First, during the post-implementation period of five years, the case-mix may have been changed, which may impact the results. Therefore, 


\section{CHAPTER 5}

univariate regression analyses for socio-demographic characteristics, hospital and measurement group (i.e. LATE POST and POST) in relation to mean pooled NMB were performed to get insight into the crude associations. Then, mean pooled NMB was adjusted for the effect of covariates using stepwise multi regression analysis [16]. If after adjustment $\mathrm{NNMB}_{\text {LATE POST }}$ has an increased value (which is represented by the beta of 'measurement group'), the change in case-mix has a negative impact on the sustain-

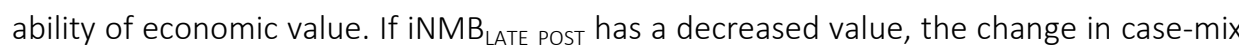
has a positive impact on the results. Second, a health care perspective instead of a societal perspective was taken to the analysis of sustainability of economic value, as costs which fall outside the health care sector may have changed over time, simply as the result of political or socio-economic changes. Third, subgroup analyses were performed on hospital level to assess potential variation between hospitals as regards sustainability of economic value, both from a societal and from a health care perspective [19]. This was done as the decision to invest in sustainability activities is made on a hospital level and not on an overall group level.

\section{Results}

\section{Patient characteristics}

A total of 160 breast cancer patients were included in the LATE POST group, of which 39 did not return the questionnaires six weeks after surgery. The patients who did not return the questionnaires did not significantly differ from those in the LATE POST group with respect to age $(p=0.612)$, type of surgery $(p=0.637)$, reoperation $(p=0.618)$ and readmission rate $(p=0.626)$. In total, data of 248 patients were included in the NMB analyses (121 LATE POST group; 127 POST group). Mean age was 60.3 years (IQR P25:53-P75:68) in the LATE POST group and was 56.1 years (IQR P25:48-P75:62) in the POST group $(p=0.001)$. Other patient characteristics were comparable between both groups (table 1). 
ANALYSING THE SUSTAINABILITY OF ECONOMIC VALUE

Table 1 Social demographic characteristics of the POST and LATE POST group (excluding missing values)

\begin{tabular}{|c|c|c|c|}
\hline & $\begin{array}{l}\text { POST group } \\
(n=127)\end{array}$ & $\begin{array}{l}\text { LATE POST } \\
(n=121)\end{array}$ & P-value \\
\hline Mean age (years) & 56 (SD 10,8) [33-89] & $60 *(S D 10,3)[39-90]$ & 0.001 \\
\hline Aged 65 years and older $(n)$ & $24(18.9 \%)$ & $44 *(36.7 \%)$ & 0.002 \\
\hline Living together with partner $(n)$ : yes & $100(78.7 \%)$ & $100(82.6 \%)$ & 0.437 \\
\hline Highest educational degree (n) & & & 0.231 \\
\hline Primary school & $4(3.2 \%)$ & $1(0.8 \%)$ & \\
\hline Secondary school & $98(77.8 \%)$ & $86(71.1 \%)$ & \\
\hline College & $19(15.1 \%)$ & $28(23.1 \%)$ & \\
\hline University & $5(4.0 \%)$ & $6(5 \%)$ & \\
\hline Paid work (n): Yes & $55(44 \%)$ & $50(41.3 \%)$ & 0.671 \\
\hline Mean hours paid work per week & 10.1 (SD 13.5) [0-40] & 10.3 (SD 14.7) [0-70] & 0.779 \\
\hline Performing housekeeping tasks (n): Yes & $72(57.1 \%)$ & $57(47.5 \%)$ & 0.130 \\
\hline Having a housekeeper (n): Yes & $26(20.6 \%)$ & $24(19.8 \%)$ & 0.902 \\
\hline Volunteer work (n): Yes & $21(16.7 \%)$ & $25(20.8 \%)$ & 0.402 \\
\hline Having children (n): Yes & $107(85.6 \%)$ & $100(82.6 \%)$ & 0.526 \\
\hline
\end{tabular}

$P<0,050$

Sustainability of economic value

\section{Costs and effectiveness}

The proportion of patients treated in short stay was $90 \%$ in the LATE POST group and $81 \%$ in the POST group $(p=0.028)$. The proportion of readmissions was significantly lower in the LATE POST group compared to the POST group ( $p=0.038$ ). The proportion of patients undergoing breast conserving surgery was $62 \%$ in the LATE POST group and $63 \%$ in the POST group ( $p=0.896$ ). The complication rate was not significantly different $(p=0.451)$. Less reoperations were observed in the LATE POST group compared to the POST group ( $p=0.010$ ). Mean pooled societal costs of SSP decreased from $€ 8013$ in the POST group to $€ 6526$ in the LATE POST group (additional file 1$)(p=0.014)$. Health care costs were comparable, amounting to $€ 4337$ in the LATE POST group and $€ 4663$ in the POST group ( $p=0.272)$. Mean pooled effectiveness was 0.089 QALY $(S E=0.002)$ in the LATE POST group and 0.086 QALY ( $S E=0.002)$ in the POST group $(p=0.514)$.

\section{Net Monetary Benefit}

Mean pooled NMB per patient was $€ 550$ (SE=418) in the LATE POST group and $€-1098$ $(\mathrm{SE}=556)$ in the POST group. The mean pooled INMB ${ }_{\text {LATE POST }}$ per patient of breast cancer 


\section{CHAPTER 5}

surgery care was $€ 1649$. This means that, at a lambda of $€ 80.000$, the economic value of SSP was fully sustained. Bootstrap analysis showed a $95 \%$ probability that the economic value of SSP was sustained also with a range lower threshold values (€0$€ 75.000)$.

\section{Secondary analyses}

Univariate regression analyses showed that age category ( $B=€ 3000, S E=€ 796, p=<0.001$ ) and 'having a paid job' ( $B=€-4497, S E=€ 642, p=<0.001$ ) were associated with the NMB from a societal perspective. As 'having a paid job' was significantly and highly correlated with age category $(r=-0.434, p=<0.001)$ and age is a more intrinsic patient characteristic, 'having a paid job' was not included in the multiple regression model. Higher age in the LATE POST group showed to have a positive impact on the economic value as the iNMB ${ }_{\text {LATE }}$ POST decreased from $€ 1649$ (SE=698, $p=0.018$ ) to $€ 1162$ (SE=714, $p=0.105$ ) after adjusting for changes in age $(B=2661, S E=821, p=0.001)$. Economic value was fully

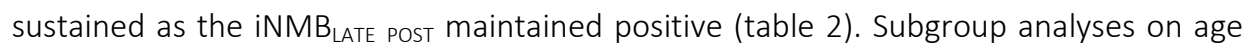
category level, i.e. patients 64 years of age or younger and patients 65 years of age or older, were performed with respect to productivity losses (total indirect costs).

From a health care perspective the iNMB $\mathrm{NATE}_{\text {POST }}$ was $€ 487$ (table 3 ) and bootstrap analysis showed a $65 \%$ probability that the economic value of SSP was sustained $(\mathrm{Cl} 95 \%$ $€$-88 - €949) irrespective of the threshold value (€0-€75.000). Age category had no significant impact on iNMB $B_{\text {LATE }}$ POST from a health care perspective $(B=€ 42, S E=527$, $p=0.937)$.

Subgroup analyses showed that the iNMB $\mathrm{BATE}_{\text {POST }}$ from a societal perspective varied on hospital level. Three hospitals showed a positive iNMB LATE POST, whereas hospital

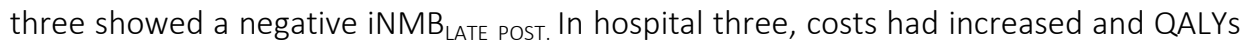
had decreased in the LATE POST group compared to the POST group.

Table 2 Stepwise multivariate regression analyses examining mean pooled NMB Dependent variable: Net Monetary Benefit (mean pooled costs $(€), n=248$ )

\begin{tabular}{lllll}
\hline Measurement & B & SE & P level & Adjusted R $^{2}$ \\
\hline Constant & -1098 & 506 & 0.031 & 0.024 \\
Measurement (LATE POST, yes/no) & 1649 & 698 & 0.018 & \\
Measurement in combination with age category & $\mathrm{B}$ & $\mathrm{SE}$ & $\mathrm{p} \mathrm{level}$ & Adjusted R \\
Constant & -1601 & 498 & 0.001 & 0.065 \\
Measurement (LATE POST, yes/no) & 1162 & 714 & 0.105 & \\
Age category ( $\geq 65$ years, yes/no) & 2661 & 821 & 0.001 & \\
\hline
\end{tabular}

$\mathrm{P}<0.050$, POST: immediately after implementation, LATE POST: five years after active implementation 


\section{ANALYSING THE SUSTAINABILITY OF ECONOMIC VALUE}

Table 3 Secondary analyses results of mean (pooled) costs, effects and net monetary benefit per patient LATE POST group in the post-implementation phase, compared to the POST group

\begin{tabular}{|c|c|c|c|c|c|}
\hline & & Costs $(€)$ & $\begin{array}{l}\text { Effectiveness } \\
\text { (QALYs) }\end{array}$ & $\begin{array}{l}\text { NMB } \\
(€)\end{array}$ & $\begin{array}{c}\text { iNMB* }^{*} \\
(€)\end{array}$ \\
\hline \multicolumn{6}{|c|}{ Hospital level (total costs from a societal perspective) } \\
\hline \multirow[t]{2}{*}{ Hospital 1} & POST (77\% SSP) & $8622(\mathrm{SE}=1199)$ & $0.081(S E=0.005)$ & $-2160(S E=1329)$ & NA \\
\hline & LATE POST (94\% SSP) & $6226(S E=592)$ & $0.087(S E=0.004)$ & $-707(\mathrm{SE}=733)$ & 1453 \\
\hline \multirow[t]{2}{*}{ Hospital 2} & POST (67\% SSP) & $6924(\mathrm{SE}=689)$ & $0.091(S E=0.005)$ & $313(S E=895)$ & NA \\
\hline & LATE POST (97\% SSP) & $6246(S E=822)$ & $0.090(S E=0.004)$ & $965(\mathrm{SE}=1055)$ & 652 \\
\hline \multirow[t]{2}{*}{ Hospital 3} & POST (93\% SSP) & $6400(\mathrm{SE}=717)$ & $0.089(S E=0.004)$ & $748(\mathrm{SE}=891)$ & NA \\
\hline & LATE POST ( $82 \%$ SSP) & $7314(\mathrm{SE}=779)$ & $0.084(S E=0.005)$ & $-626(S E=878)$ & -1374 \\
\hline \multirow[t]{2}{*}{ Hospital 4} & POST ( $85 \%$ SSP) & $9505(\mathrm{SE}=892)$ & $0.086(S E=0.005)$ & $-2505(S E=1052)$ & NA \\
\hline & LATE POST (92\% SSP) & $6178(\mathrm{SE}=548)$ & $0.095(S E=0.002)$ & $1427(\mathrm{SE}=599)$ & 3932 \\
\hline \multicolumn{6}{|c|}{ Health care perspective } \\
\hline & POST ( $81 \%$ SSP) & $4663(\mathrm{SE}=267)$ & $0.086(S E=0.002)$ & $2252(\mathrm{SE}=340)$ & NA \\
\hline & LATE POST (90\% SSP) & $4337(\mathrm{SE}=111)$ & $0.089(S E=0.002)$ & $2739(\mathrm{SE}=213)$ & 487 \\
\hline \multicolumn{6}{|c|}{ Hospital level (total costs from a health care perspective) } \\
\hline \multirow[t]{2}{*}{ Hospital 1} & POST (77\% SSP) & $5379(\mathrm{SE}=734)$ & $0.081(S E=0.005)$ & $1082(\mathrm{SE}=882)$ & \\
\hline & LATE POST (94\% SSP) & $4325(\mathrm{SE}=229)$ & $0.087(S E=0.004)$ & $2607(S E=415)$ & 1525 \\
\hline \multirow[t]{2}{*}{ Hospital 2} & POST (67\% SSP) & $4316(S E=262)$ & $0.091(S E=0.005)$ & $2922(S E=548)$ & \\
\hline & LATE POST (97\% SSP) & $4114(\mathrm{SE}=275)$ & $0.090(S E=0.004)$ & $3097(\mathrm{SE}=492)$ & 175 \\
\hline \multirow[t]{2}{*}{ Hospital 3} & POST (93\% SSP) & $3474(\mathrm{SE}=207)$ & $0.089(S E=0.004)$ & $3675(\mathrm{SE}=432)$ & \\
\hline & LATE POST ( $82 \%$ SSP) & $4782(\mathrm{SE}=164)$ & $0.084(S E=0.005)$ & $1906(\mathrm{SE}=410)$ & -1769 \\
\hline \multirow[t]{2}{*}{ Hospital 4} & POST (85\% SSP) & $5135(S E=404)$ & $0.086(S E=0.005)$ & $1864(\mathrm{SE}=588)$ & \\
\hline & LATE POST (92\% SSP) & $4013(\mathrm{SE}=199)$ & $0.095(S E=0.002)$ & $3592(\mathrm{SE}=310)$ & 1728 \\
\hline \multicolumn{6}{|c|}{ Age category (total indirect costs) } \\
\hline \multirow[t]{2}{*}{$<65$ years } & POST $(n=103,81 \%)$ & $3386(\mathrm{SE}=369)$ & $0.086(\mathrm{SE}=0.003)$ & $3483(\mathrm{SE}=454)$ & \\
\hline & LATE POST $(n=24,19 \%)$ & $2817(S E=444)$ & $0.089(\mathrm{SE}=0.006)$ & $3950(\mathrm{SE}=506)$ & 467 \\
\hline \multirow[t]{2}{*}{$\geq 65$ years } & POST $(n=76,63 \%)$ & $942(S E=882)$ & $0.085(S E=0.003)$ & $6167(S E=1054)$ & \\
\hline & LATE POST $(n=44,37 \%)$ & $215(\mathrm{SE}=69)$ & $0.095(\mathrm{SE}=0.003)$ & $7392(S E=208)$ & 1225 \\
\hline
\end{tabular}

NMB: net monetary benefit, POST: measurement immediately after implementation, LATE POST: measurement five years after active implementation, $*$ iNMB ${ }_{\text {LATE POST }}=$ NMB LATE POST $_{-}$NMB POST 


\section{CHAPTER 5}

These differences in iNMB $\mathrm{BATE}_{\text {POST }}$ between hospitals were also observed from a health care perspective. Hospital one, two and four showed sustained economic value results while the iNMB $\mathrm{NATE}_{\text {POST }}$ in hospital three was $€-1769$.

As the higher age in the LATE POST group positively impacted on the societal NMB only (not on health care NMB), we additionally performed a subgroup analyses distinguishing patients younger than 65 and patients aged 65 and older with respect to NMB based on indirect costs (mainly productivity losses). The iNMB group aged 65 and older and $€ 467$ in the group of patients younger than 65 . This difference was mainly explained by lower productivity costs in the group aged 65 and older.

\section{Discussion}

In the current paper the NMB approach was applied for the analysis of sustainability of economic value in health care. The approach builds on the framework for assessing the value of implementation [12]. Application of the NMB approach was demonstrated by calculating the level of sustained economic value of a short stay programme for breast cancer surgery care. The results showed that the overall economic value achieved early after implementation was fully sustained five years following implementation. Additional analyses were performed to further explore the concept of sustainability of economic value.

Some interesting issues were revealed when analysing the sustainability of economic value of an innovation. First, the current study showed that patients were significantly older in the LATE POST group compared to the POST group. The iNMB decreased slightly in the LATE POST group compared to the POST group after adjustment for age category. Although this result seems contra intuitive, it can be explained by the increased proportion of women 65 years of age and older and by lower productivity costs in this group. Second, when changing the societal perspective into a health care perspective, the economic value (iNMB LATE POST) was lower but still positive. Age showed to have no impact on the iNMB from the health care perspective. Third, subgroup analyses on hospital level, showed wide variation in the level of sustained economic value, with one hospital showing a negative iNMB. In hospital three the iNMB from the societal perspective was $€-1374$ in the LATE POST group compared to the POST group and $€$ 1796 from a health care perspective. This may imply that additional investment in sustainability activities may be worthwhile in this hospital.

\section{Reflection on the concept of sustainability of economic value}

The current study showed that the breast cancer patients undergoing surgery were on average four years older, five years following implementation compared to early postimplementation results. However, in the Netherlands mean age for all breast cancer patients undergoing surgery increased only slightly from 60.1 years (IQR P25:50-P75:70) 
in 2007 to 60.7 years (IQR P25:51-P75:70) in 2012 (Netherlands Cancer Registry). The age difference as shown in the current study cannot be explained by a difference in inclusion criteria because these were exactly the same over the two measurement moments. Hence, the national data implies some selection bias in the primary implementation study. Despite the increase in age, the proportion of patients treated in short stay had nevertheless increased from $81 \%$ to $90 \%$. Long-term experience with SSP - given the inclusion criteria - may have influenced the application of the programme to older and more complex patients. Consequently, an extended learning process of SSP in the post-implementation phase may have resulted in increased economic value. It is therefore questionable if regression analyses are needed to adjust for changes in case-mix over time, as it may well be a characteristic of sustainability.

The health care context is dynamic and is suggested to be a determinant of sustainability [20]. Furthermore, some authors suggest to focus more on macro socioeconomic and political level influences in future sustainability research [21, 22]. Thus, another question is whether or not sustainability of economic value must be studied from a health care or from a societal perspective. The current study showed that the proportion of patients having a paid job did not significantly change over time. In the Netherlands however, the unemployment figure increased from $4.5 \%$ in 2007 to $6.4 \%$ in 2012 and the intention of women to extent the work carrier till 65 years increased from $21.1 \%$ in 2005 to $44.0 \%$ in 2010 [23], which - in theory - might have impacted the indirect costs. The societal perspective encompasses a broad range of macro socioeconomic and political level influences and may have influenced the results. Using a sustainability evaluation timeframe of for example five years, the change in costs outside the health care sector over time may not be related to (changes in) the innovation's characteristics or performance. Furthermore a societal perspective is not always desired as some countries prefer to only consider information relevant from a local or national budget holder's perspective [24].

Subgroup analyses of the current study on hospital level showed substantial variation in the level of sustained economic value. The results imply that an individual hospital level analysis may be more appropriate for analysing the sustainability of economic value than an analysis on a group level. Moreover, the amount and the type of activities to sustain an innovation may differ between settings. Unfortunately, we did not collect data prospectively regarding the actually performed sustainability activities over the period of five years, so these costs may have been missed. Nevertheless, qualitative research showed that the professionals involved did not perceive that they executed promising activities to sustain the short stay programme in real-world [25].

Following the NMB approach for analysing the sustainability of economic value, optimal implementation effects are assumed immediately after implementation, at the moment when additional investment are supposed to have no added value. The results presented in the current study suggest that a normalisation effect in terms of increased uptake may have taken place in the post-implementation phase and that implementa- 


\section{CHAPTER 5}

tion effects were measured too soon following the implementation activities. This raises the question what the best moment is to determine the optimal implementation level; this level should be used as the reference point for analysing sustainability of economic value. If sustainability is measured too soon after implementation, optimal implementation of an innovation may not yet be reached. If sustainability is measured too late after implementation, the momentum for investment in sustainability activities may have passed. In some cases, more simple sustainability evaluations, for example related to clinical outcomes or professionals' adherence to the new desired behaviour, may be an indicator for sustainability of economic value. To prevent inefficient assessment of sustainability of economic value, more simple sustainability evaluations need to be considered and explored.

Finally, sustainability of economic value needs to be distinguished from the costeffectiveness of an innovation. If the economic value of an innovation has not been fully sustained compared to the optimal implementation situation, current practice may still be cost-effective compared to the pre-implementation situation, which in this case was the former clinical admission practice in breast cancer patients.

\section{Conclusion}

International trends as changing socio-demographic characteristics and further advances in health care lead to an increased demand for care. Sustainability of economic value of cost-effective innovations has impact on the efficiency of the health care system. This was, to our knowledge, the first study focusing on sustainability of health care innovations from an economic perspective. The current study applied the NMB approach for the analysis of sustainability of economic value in practice. Results showed that the economic value had sustained five years following implementation of a short stay programme for breast cancer surgery. The changed case-mix in terms of age revealed to have a positive impact on the sustainability of economic value, mainly as a result of decreased productivity costs. Economic value was not sustained in one of the four early adopter hospitals. Application of the NMB approach was useful for the analysis of sustainability of economic value. The current study discussed some issues, such as whether regression analysis should be performed to correct for differences in case-mix over time, the appropriate perspective, the level of analysis (group- or individual hospital level), and the timing of the analysis. Future research should further explore how sustainability of economic value should be analysed. 


\section{References}

1. Ruwaard D SM, Kroese M, Steevens J, Ouwens M, Hilbrink M. Triple Aim en de toepassing in de regionale proeftuinen 'Populatiemanagement' van VGZ en CZ. Datawyse / Universitaire Pers Maastricht: Department of Health Services Research, Maastricht University/ IQ healthcare, Radboudumc; 2014.

2. Berwick DM, Nolan TW, Whittington J. The triple aim: care, health, and cost. Health affairs (Project Hope). 2008 May-Jun;27(3):759-69.

3. R. Grol MW, M. Eccles, D. Davis. Improving patient care: the implementation of change in health care. John Wiley \& Sons; 2013.

4. Shediac-Rizkallah MC, Bone LR. Planning for the sustainability of community-based health programs: conceptual frameworks and future directions for research, practice and policy. Health Educ Res. 1998 Mar;13(1):87-108.

5. NHS. Sustainability and its relationship with spread and adoption, General improvement skills. Coventry, UK: Improvement Leaders' guide; 2007

6. Ament SMC, Gillissen F, Maessen JMC, Dirksen CD, van der Weijden T, von Meyenfeldt MF. Sustainability of healthcare innovations (SUSHI): long term effects of two implemented surgical care programmes (protocol). BMC health services research. 2012;12:423.

7. Stirman SW, Miller CJ, Toder K, Calloway A. Development of a framework and coding system for modifications and adaptations of evidence-based interventions. Implement Sci. 2013;8:65.

8. Scheirer MA. Is Sustainability Possible? A Review and Commentary on Empirical Studies of Program Sustainability. American Journal of Evaluation. 2005 September 2005;26(3):27.

9. Graham ID, Logan J, Harrison MB, Straus SE, Tetroe J, Caswell W, et al. Lost in knowledge translation: time for a map? J Contin Educ Health Prof. 2006 Winter;26(1):13-24.

10. Tricco AC, Cogo E, Ashoor H, Perrier L, McKibbon KA, Grimshaw JM, et al. Sustainability of knowledge translation interventions in healthcare decision-making: protocol for a scoping review. BMJ Open. 2013 January 1, 2013;3(5).

11. Porter ME. What Is Value in Health Care? New England Journal of Medicine. 2010;363(26):2477-81.

12. Fenwick $E$, Claxton $K$, Sculpher $M$. The value of implementation and the value of information: combined and uneven development. Medical decision making : an international journal of the Society for Medical Decision Making. 2008 Jan-Feb;28(1):21-32.

13. Hoomans T, Ament AJHA, Evers SMAA, Severens JL. Implementing guidelines into clinical practice: what is the value? Journal of Evaluation in Clinical Practice. 2011;17(4):606-14.

14. de Kok M, van der Weijden T, Voogd AC, Dirksen CD, van de Velde CJ, Roukema JA, et al. Implementation of a short-stay programme after breast cancer surgery. Br J Surg. 2010 Feb;97(2):189-94.

15. Ament SMC, Gillissen F, Maessen JMC, Dirksen CD, Bell AVRJ, Vissers YL, et al. Sustainability of short stay after breast cancer surgery in early adopter hospitals. Breast (Edinburgh, Scotland). 2014.

16. Hoch JS, Briggs AH, Willan AR. Something old, something new, something borrowed, something blue: a framework for the marriage of health econometrics and cost-effectiveness analysis. Health economics. 2002 Jul;11(5):415-30.

17. Tambour M, Zethraeus N, Johannesson M. A note on confidence intervals in cost-effectiveness analysis. International journal of technology assessment in health care. 1998 Summer;14(3):467-71.

18. Efron B. Introduction to the bootstrap. New York, USA: Chapmand \& Hall; 1993.

19. Thabane L, Mbuagbaw L, Zhang S, Samaan Z, Marcucci M, Ye C, et al. A tutorial on sensitivity analyses in clinical trials: the what, why, when and how. BMC medical research methodology. 2013;13:92.

20. Gruen RL, Elliott JH, Nolan ML, Lawton PD, Parkhill A, McLaren CJ, et al. Sustainability science: an integrated approach for health-programme planning. Lancet. 2008 Nov 1;372(9649):1579-89.

21. Doyle C, Howe C, Woodcock T, Myron R, Phekoo K, McNicholas C, et al. Making change last: applying the NHS institute for innovation and improvement sustainability model to healthcare improvement. Implementation Science. 2013;8(1):127.

22. Scheirer MA, Dearing JW. An agenda for research on the sustainability of public health programs. American journal of public health. 2011 Nov;101(11):2059-67. 


\section{CHAPTER 5}

23. Centraal Bureau vo de Statistiek. StatLine 2014 [cited 2014 September 05].

24. Mauskopf JA, Sullivan SD, Annemans L, Caro J, Mullins CD, Nuijten M, et al. Principles of Good Practice for Budget Impact Analysis: Report of the ISPOR Task Force on Good Research Practices-Budget Impact Analysis. Value in Health. 2007;10(5):336-47.

25. Ament SMC, Gillissen F, Moser A, Maessen JMC, Dirksen CD, van der Weijden T, et al. What determines sustainability of two quality improvement programs after achieving early implementation success? A qualitative case study. BMC health services research. 2014;submitted. 
Additional file 1

Mean pooled costs and effects in the POST and LATE POST group

\begin{tabular}{|c|c|c|c|c|c|c|c|}
\hline & \multicolumn{3}{|l|}{ POST } & \multicolumn{3}{|c|}{ LATE POST } & \multirow{2}{*}{$\begin{array}{c}\text { Differences in } \\
\text { costs }(€)(95 \% \mathrm{CI}) \\
\text { LATE POST - POST }\end{array}$} \\
\hline & volume & $\begin{array}{c}\text { costs per } \\
\text { patient }\end{array}$ & $\begin{array}{l}\% \text { of } \\
\text { total }\end{array}$ & volume & $\begin{array}{c}\text { costs per } \\
\text { patient }\end{array}$ & $\begin{array}{l}\% \text { of } \\
\text { total }\end{array}$ & \\
\hline \multicolumn{8}{|c|}{ Direct $\mathrm{HC}$ costs before admission } \\
\hline Tests and procedures & n.a. & 675.28 & 8.2 & n.a. & 782.81 & 12.0 & \\
\hline MDO & 0.94 & 74.53 & 0.9 & 0.96 & 76.23 & 1.7 & \\
\hline Outpatient clinic & 3.09 & 257.91 & 3.2 & 3.15 & 268.85 & 4.1 & \\
\hline Preoperative screening & 0.75 & 37.61 & 0.5 & 0.92 & 46.38 & 0.7 & \\
\hline Subtotal & & 1045 & 13.0 & & 1174 & 17.0 & $-77(-172,13)$ \\
\hline \multicolumn{8}{|c|}{ Direct $\mathrm{HC}$ costs during admission } \\
\hline Wire localisation & 0.4 & 131.58 & 1.6 & 0.42 & 137.9 & 2.1 & \\
\hline Surgery & n.a. & 1628.95 & 20.3 & n.a. & 1707.19 & 26.2 & \\
\hline Admission period & 0.98 & 500.88 & 6.3 & 0.82 & 443.96 & 6.8 & \\
\hline Subtotal & & 2261 & 28.2 & & 2272 & 34.8 & $-76(-169,19)$ \\
\hline \multicolumn{8}{|c|}{ Direct $\mathrm{HC}$ costs after admission } \\
\hline MDO & 0.97 & 91.62 & 1.1 & 0.91 & 86.53 & 1.3 & \\
\hline Outpatient clinic & 1.9 & 148.35 & 1.9 & 2.29 & 191.5 & 2.9 & \\
\hline Surgery incl SN & n.a. & 361.74 & 4.5 & n.a. & 180.99 & 2.8 & \\
\hline Readmission period & 0.78 & 406.67 & 5.1 & 0.28 & 46.09 & 0.7 & \\
\hline Emergency departement & 0.14 & 22.95 & 0.3 & 0.03 & 4.47 & 0.1 & \\
\hline GP practice & 1.17 & 27.42 & 0.3 & 0.51 & 11.96 & 0.2 & \\
\hline GP home & 0.56 & 26.24 & 0.3 & 0.35 & 16.33 & 0.3 & \\
\hline GP telephone & 0.44 & 5.23 & 0.1 & 0.42 & 4.99 & 0.08 & \\
\hline GP post & 0.06 & 4.54 & 0.1 & 0.04 & 2.77 & 0.04 & \\
\hline Home care, domestic help & 1.78 & 49.22 & 0.6 & 0.3 & 7.74 & 0.1 & \\
\hline Home care, nursing & 2.99 & 138.87 & 1.7 & 2.03 & 96.4 & 1.5 & \\
\hline Paramedic care (various) & n.a. & 12.11 & 0.2 & n.a. & 32.03 & 0.5 & \\
\hline Medication (various) & n.a. & 49.86 & 0.6 & n.a. & 193.98 & 3.0 & \\
\hline Pharmacist's fee & 0.94 & 6.19 & 0.1 & 1.29 & 8.45 & 0.1 & \\
\hline Other & n.a. & 5.11 & 0.1 & n.a. & 6.41 & 0.1 & \\
\hline Subtotal & & 1356 & 16.9 & & 891 & 13.7 & $519(120,1056)$ \\
\hline SUBTOTAL HC costs & & 4663 & 58.2 & & 4337 & 66.5 & $351(-88,949)$ \\
\hline
\end{tabular}




\begin{tabular}{|c|c|c|c|c|c|c|c|}
\hline & \multicolumn{3}{|l|}{ POST } & \multicolumn{3}{|c|}{ LATE POST } & \multirow{2}{*}{$\begin{array}{c}\text { Differences in } \\
\text { costs (€) }(95 \% \mathrm{Cl}) \\
\text { LATE POST - POST }\end{array}$} \\
\hline & volume & $\begin{array}{c}\text { costs per } \\
\text { patient }\end{array}$ & $\begin{array}{l}\% \text { of } \\
\text { total }\end{array}$ & volume & $\begin{array}{c}\text { costs per } \\
\text { patient }\end{array}$ & $\begin{array}{l}\% \text { of } \\
\text { total }\end{array}$ & \\
\hline \multicolumn{8}{|l|}{ Direct non $\mathrm{HC}$ costs } \\
\hline Informal care & 28.97 & 279.8 & 3.5 & 17.55 & 169.6 & 2.6 & \\
\hline Paid housekeeper & 1.92 & 18.5 & 0.2 & 1.37 & 13.2 & 0.2 & \\
\hline Meal service & 0.16 & 0.95 & 0.0 & 0 & 0 & 0.0 & \\
\hline Total & & 299 & 3.7 & & 183 & 2.8 & $170(1,381)$ \\
\hline \multicolumn{8}{|l|}{ Indirect costs } \\
\hline Sick leave & 56.54 & 2398.3 & 29.9 & 40.7 & 1726.5 & 26.5 & \\
\hline Loss of voluntary work & 5.82 & 56.2 & 0.7 & 3.23 & 31.2 & 0.5 & \\
\hline Loss of leisure time & 6.7 & 64.71 & 0.8 & 3.86 & 37.3 & 0.6 & \\
\hline Loss of household tasks & 40.46 & 390.8 & 4.9 & 10.73 & 103.7 & 1.6 & \\
\hline other & n.a. & 14.3 & 0.2 & n.a. & 16.1 & 0.3 & \\
\hline Subtotal & & 2924 & 36.5 & & 1915 & 29.3 & $1079(233,1978)$ \\
\hline \multicolumn{8}{|l|}{ Out of pocket costs } \\
\hline Alternative treatment & 0,12 & 6.96 & 0.1 & 0 & 0 & 0.00 & \\
\hline Aids bought & n.a. & 64.67 & 0.8 & n.a. & 65.7 & 1.00 & \\
\hline Travelling expenses & n.a. & 1.79 & 0.00 & n.a. & 0.91 & 0.01 & \\
\hline Other & n.a. & 52.69 & 0.7 & n.a. & 24.43 & 0.37 & \\
\hline Subtotal & & 126 & 1.6 & & 91 & 1.4 & $87(-4,180)$ \\
\hline SUBTOTAL non HC costs & & 3350 & 41.8 & & 2189 & 33.5 & $1223(285,2153)$ \\
\hline Total costs & & 8013 & & & 6526 & & $1522(414,2664)$ \\
\hline
\end{tabular}

POST: immediately after implementation; LATE POST: five years after active implementation; HC: health care 


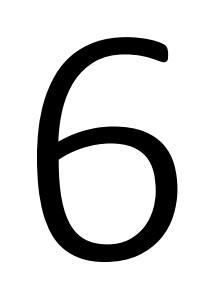

Sustainability of professionals' adherence to guideline recommendations in medical practice: a systematic review

Stephanie M C Ament, Jeanny J de Groot, José M C Maessen, Carmen D Dirksen,

Trudy van der Weijden, Jos Kleijnen

Submitted 


\section{CHAPTER 6}

\section{Abstract}

\section{Objective}

The aim of this systematic review was to evaluate the sustainability of professionals' adherence to guideline recommendations in medical practice more than one year following the cessation of active implementation.

\section{Design}

Systematic review

\section{Data sources}

Searches were conducted till February 2014 in MEDLINE, CINAHL, EMBASE, Cochrane Central Register of Controlled Trials (CENTRAL) and the Guidelines International Network (GIN) library. Before final analyses, update searches were performed to identify possible relevant studies (June 26, 2014). A snowball strategy, in which reference sections of other reviews and of included papers were searched, was used to identify additional papers.

\section{Eligibility criteria}

Studies needed to be focused on sustainability and on professionals' adherence to clinical practice guidelines in medical care. Studies had to include at least two measurements: one before (PRE) or immediately after implementation (EARLY POST) and one measurement longer than one year after active implementation (LATE POST).

\section{Results}

The search retrieved 3950 items, of which thirteen studies met the inclusion criteria, involving seventeen sustainability evaluations. Eleven studies used a case series design and two studies used a randomised controlled trial design. Implementation activities varied and included professional targeted interventions, organisational interventions and financial interventions. In five studies external experts facilitated the implementation strategy. In one study it was unclear whether the implementation strategy was externally supported. The mean timeframe between the end of active implementation and the sustainability evaluation was 2.7 years [ $\min 1.5-\max 7.0$ ]. The studies were heterogeneous with respect to their methodology. Sustainability was considered to be successful if performance in terms of professionals' adherence was fully maintained in the late post-implementation phase. Long-term sustainability of professionals' adherence was reported in $41 \%$ of the evaluations (7 out of 17), adherence was not sustained in five evaluations, four evaluations showed mixed sustainability results and in one evaluation it was unclear whether the professional adherence was sustained. Professionals' adherence was sustained at least at a level of 90\%, in 53\% (9 out of 17) of the evaluations. 


\section{Conclusions}

Professionals' adherence to a clinical practice guideline in medical care decreased after more than one year after implementation in about half of the cases. Due to the limited number of studies, the absence of a uniform definition, the high risk of bias, and the mixed results of studies, no firm conclusion about the sustainability of professionals' adherence to guideline recommendations in medical practice can be drawn. More sustainability evaluations are needed. 


\section{Introduction}

More than ever, improving health care performance is necessary due to limited budgets, increased demand and the continuous development of innovations. Quality of care can be improved by decreasing unwarranted practice variation between professionals. One way to reduce practice variation is by transferring evidence-based knowledge into daily practice. To facilitate the translation of the most recent evidence into practice, guidelines are developed and implemented. Following the Institute of Medicine (IOM), clinical practice guidelines are "statements that include recommendations intended to optimize patient care that are informed by a systematic review of evidence and an assessment of the benefit and harms of alternative care options" [1]. Guidelines contain practical evidence based advice for professionals and patients and aim to improve the quality of care [2]. In general, uptake of guidelines does not happen spontaneously and often an active implementation approach is required [3]. Moreover, once a guideline is successfully implemented in practice, it may be difficult to sustain the quality improvements over a longer period of time. People tend to fall back into old routines [4], which may impact long-term adherence to a guideline.

The road towards sustainability of health care innovations into practice is suggested to be a dynamic process [5] and sustainable adherence may not be self-evident without continued efforts. Sustainable change of professionals' behaviour has the potential to result in more optimal health care delivery and efficiency. Not sustaining quality improvements can result in nihilistic attitudes towards future innovation. In recent years, sustainability has gained attention in health care. Unfortunately, the concept of sustainability is still underdeveloped $[6,7]$. Some existing reviews studied sustainability from a wide health care perspective, including studies varying from medical care to public health. Results showed that determinants of sustainability varied widely between health care areas $[8,9]$ and suggest that partial sustainability of health care innovations is more common than full sustainability [10].

In this systematic review, the scope of sustainability research will be narrowed to professionals' adherence to clinical practice guidelines in medical care. The aim of the current review was to evaluate the level of sustained professionals' adherence to guideline recommendations in medical practice more than one year following the cessation of the implementation project.

\section{Methods}

\section{Eligibility criteria}

Studies needed to be focused on sustainability and on clinical practice guidelines. Sustainability was described as "Sustainability of change exists when a newly implemented innovation continues to deliver the benefits achieved over a longer period of time, cer- 
tainly does not return to the usual processes and becomes 'the way things are done around here' [11], even after the implementation project is no longer actively carried out, until a better innovation comes along" [12]. Studies had to include at least two measurements: one before (PRE) or immediately after implementation (EARLY POST) and one measurement longer than one year after active implementation (LATE POST). All activities to facilitate the adherence to clinical practice guidelines were labelled as part of the implementation project. Studies needed to be focused on professionals' adherence to a clinical practice guideline. Studies only using self-reported adherence were excluded to reduce the chance of social desirability bias and an overestimation of results [13]. Lastly, studies had to focus on medical care. Participants had to be health care professionals who deliver direct patient care. There were no restrictions on study design of the research articles.

\section{Search methods for identification of studies}

\section{Electronic searches}

We searched MEDLINE (OvidSP) (1946- February 2014), CINAHL (EBSCO Host) (1982February 2014), EMBASE (OvidSP) (February 2014), Cochrane Central Register of Controlled Trials (CENTRAL) and the Guidelines International Network (GIN) library for studies. The electronic search strategy was designed to focus on sustainability of guideline recommendations. Free text terms and MeSH terms regarding sustainability, quality improvement, impact and guideline recommendations were used. An information expert checked the developed search strategies (additional file 1). Before final analyses, update searches were performed to identify possible additional studies (June 26, 2014).

\section{Searching other resources}

A snowball strategy was performed, in which the reference sections of reviews [6-10, $14]$ and research papers on sustainability $[15,16]$ were searched. Also, databases such as PubMed and the Web of Knowledge Science Citation Index were used to locate publications and publications citing the original references. The process was repeated for any new relevant publication found.

\section{Data collection and analysis}

\section{Selection of studies}

All records were merged into a bibliographic database and screened independently by two reviewers (SA, JdG) based on title and abstract. Full text screening was performed by two reviewers $(S A, J d G)$. Disagreement on selection was resolved in consensus meetings with a third reviewer (TVW). Reasons for exclusion were documented during the full text screening. If more clarification or details of a study were needed, an author was contacted. Authors of conference abstracts were emailed and were asked to send the 
research protocol. Duplicate papers were identified and all papers published on one study were used for retrieving information.

\section{Data extraction and management}

Data of the methodology and results were independently extracted by two reviewers (SA, JdG), guided by a predefined data extraction form. Effective Practice and Organisation of Care (EPOC) Data Collection Checklist [17] items (e.g. location of care, type of targeted behaviour, implementation interventions) were integrated in the data extraction form. The data extraction form was developed by the authors and was pilot tested. The following study characteristics were recorded: study design, publication year, whether the study was executed in a single centre or in multiple centres, type of targeted behaviour, location of care, the name of the clinical practice guideline, clinical specialty, the implementation activities used and whether or not the implementation strategy was externally guided. An externally guided implementation strategy is a strategy which is led and supported by an external expert organisation. With respect to the methodology of the sustainability evaluation the following data were extracted: the timeframe between the end of the implementation strategy and the sustainability evaluation, the applied definition of sustainability, the data collection method, whether the evaluation was performed on patient, hospital or multiple hospital level and whether the sustainability evaluation was performed on single or multiple centre level. With respect to the outcome measures of the studies, data on the professionals' adherence rates before, early after implementation and longer than one year after implementation, and the authors' comments with respect to the sustainability of professionals' adherence were extracted. Adherence was presented in terms of proportion of patients receiving treatment according to the clinical practice guideline recommendations. If sustainability of professionals' adherence to a clinical practice guideline was evaluated at multiple post-implementation moments, the last evaluation was selected as LATE POST measurement. The authors (SA and JdG) checked if updates of the clinical practice guidelines had become available in the post-implementation phase (e.g. between the EARLY POST and the LATE POST measurement), which may explain reduced professionals' adherence. Disagreement on data extraction was resolved in consensus meetings with a third reviewer (TvW).

\section{Assessment of risk of bias in included studies}

Risk of bias assessment was independently conducted by two authors using the Downs and Black checklist for randomized and non-randomized studies [18]. This is a checklist which can be used for checking the risk of bias of original research articles of various study designs. Results were interpreted under consideration of risk of bias. The assessments were also used for recommendations for further research by identifying elements of studies that can be improved in future studies. The checklist was adapted to 
the research question. Risk of bias of the studies was presented on reporting, external validity, internal validity (bias and confounding), power and overall level.

\section{Analysis}

The analysis was narrative. This included a summary of the methodological characteristics of the sustainability evaluations, and the level of sustained professionals' adherence compared to results achieved immediately after implementation. Sustainability was considered to be successful if performance in terms of professionals' adherence was fully maintained in the late post-implementation phase. A sensitivity analysis was performed by applying a $90 \%$ instead of $100 \%$ adherence criterion of sustainability.

\section{Results}

\section{Description of studies}

For this review, 3950 items were retrieved and screened based on title and abstract, and 174 studies were assessed based on full text reading. Figure 1 shows the study selection process as recommended by the PRISMA statement [19] (figure 1) (additional file 2). Thirteen studies met the inclusion criteria for this review, describing seventeen sustainability evaluations [20-32]. Table 1 presents the characteristics of the included studies. Two publications were published before and eleven after 2000 [21, 31]. In five studies the targeted behaviour was prescribing $[22,23,26,28,31]$, in four studies procedures $[27,29,30,32]$, in three studies general management of a problem [20, 24, 25] and in one study [21] general management of a problem and prescribing. The location of care was inpatient in six studies [21, 26, 27, 30,32], outpatient in three studies [22$24]$ and mixed in five studies $[20,25,28,29,31]$.

The implementation strategy was described in twelve studies (additional file 3) [20-25, 27-37]. According to the EPOC checklist classification, in one study [22], a single element implementation strategy was executed while in the other eleven studies a multifaceted implementation strategy was executed. Implementation activities were professional targeted interventions $(n=11)[20,21,23-25,27,28,30-37]$, followed by organisational interventions $(n=6)[20,21,24,31-34,36,37]$ and financial interventions $(n=1)$ [25]. In five studies the implementation strategy was facilitated by external experts [20, $23-25,29]$. In one study it was unclear whether the implementation strategy was externally supported [26]. 


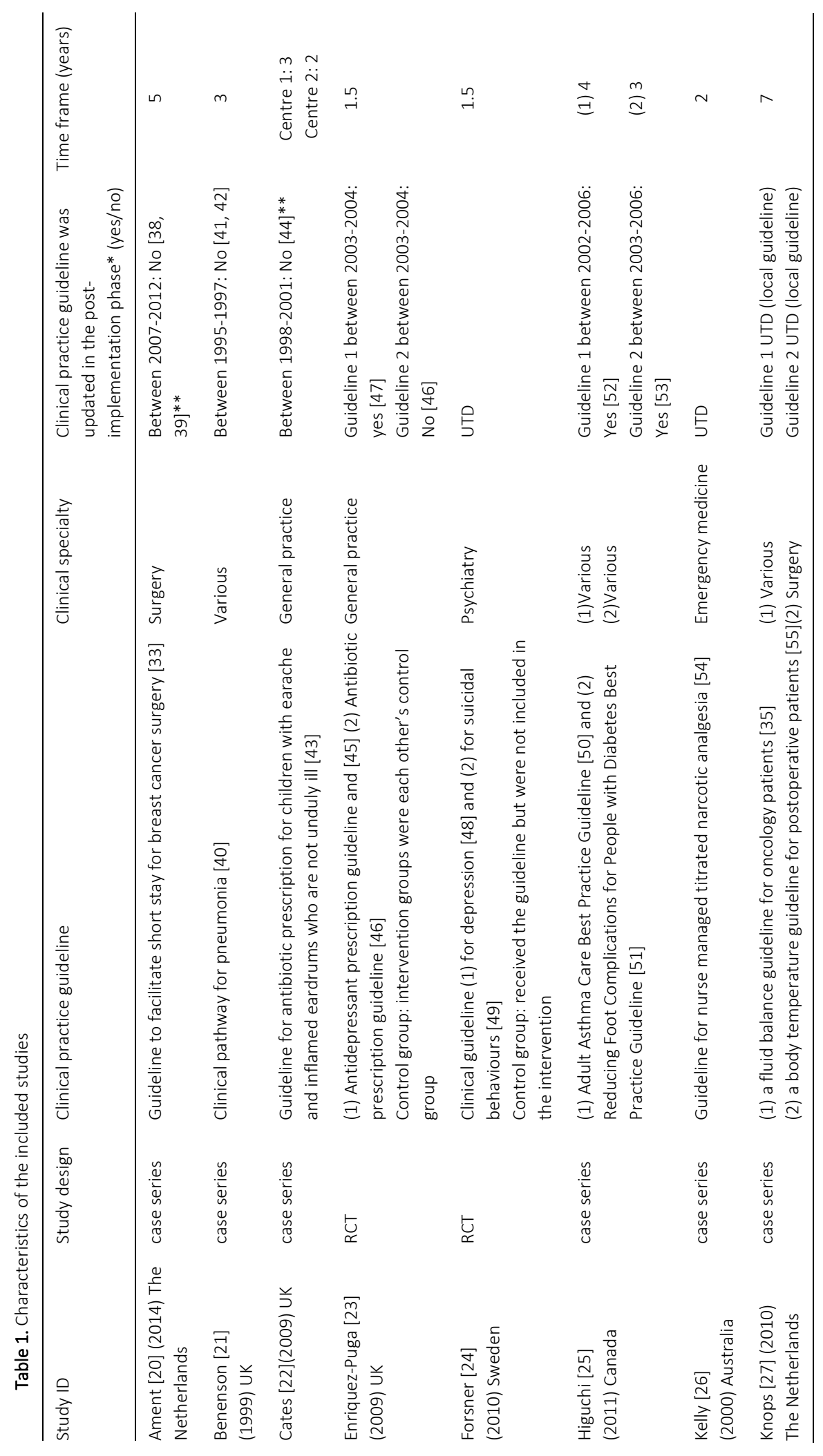




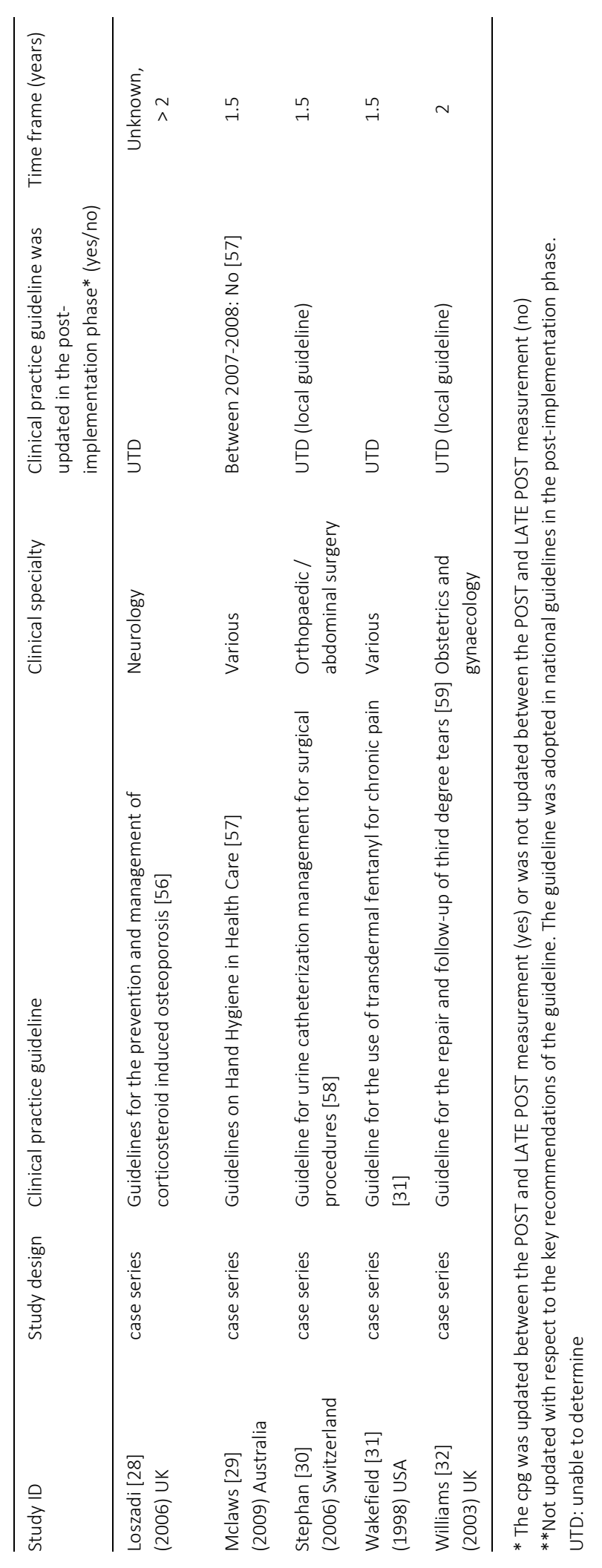




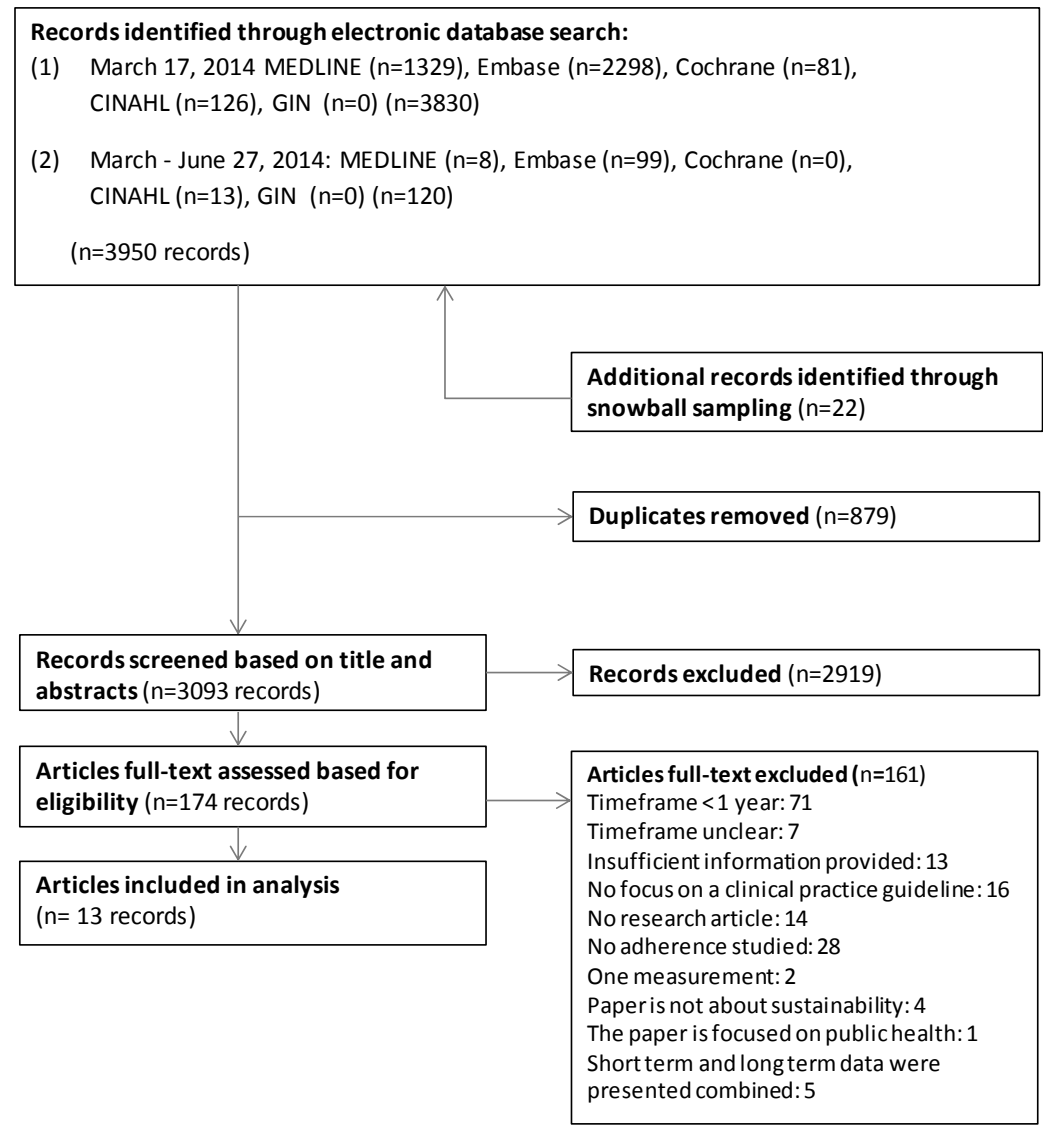

Figure 1. PRISMA Flow Diagram

\section{Characteristics of the sustainability evaluations}

The mean timeframe between the end of the implementation strategy and the sustainability evaluation of twelve studies was 2.7 years [ $\min 1.5$ - max 7.0]. The actual timeframe of one evaluation was unclear, but was at least two years [28]. Two studies referred to a definition of sustainability $[20,25]$. Eight studies used a retrospective data collection method [21-27, 31], two studies used a prospective data collection method $[29,30]$ and three studies used both a prospective and a retrospective data collection method $[20,28,32]$. Nine papers reported the level of sustained adherence of a single clinical practice guideline [20-22, 26, 28-32], while four reported the late postimplementation adherence of two clinical practice guidelines [23-25, 27]. Seven studies had a single centre design [21, 26-28, 30-32] and six studies evaluated sustainability in multiple centres $[20,22-25,29]$. Three out of six multiple centre studies evaluated the sustainability on multiple centre level $[20,24,29]$. Two out of six multiple centre studies evaluated the sustainability of professionals' adherence of two guidelines which were implemented in one centre each $[22,25]$. 


\section{Sustainability of changed behaviour}

The level of professionals' adherence was fully sustained in seven out of seventeen evaluations (table 2, additional file 4). The adherence was not fully sustained in five evaluations and four evaluations showed mixed sustainability results in the LATE POST measurement compared to the EARLY POST measurement. In one study, the EARLY POST measurement was not executed, while the authors reported sustained results [26]. After decreasing the sustainability level of professionals' adherence to $90 \%$ or higher, nine out of seventeen evaluations showed sustained results, two evaluations showed no sustained results, four evaluations showed mixed results. In two evaluations it was unclear whether the professionals' adherence had been sustained at a level 90\% or higher.

Five of the nine papers that reported about a single clinical practice guideline presented sustained professionals' adherence to clinical practice guidelines in the LATE POST measurement $[20-22,28,32]$. One of these five papers evaluated the sustainability of a single clinical practice guideline in two centres [22]. In both centres professionals' adherence had improved in the LATE POST measurement compared to the EARLY POST measurement. The four studies analysing the sustainability of two clinical practice guidelines showed mixed results. Two of these four studies [23, 27], presented the same level or improved adherence to one guideline and decreased adherence to the other guideline in the LATE POST measurement compared to the EARLY POST measurement. The other two of these four studies [24, 25] presented adherence results on guideline recommendation level and did not present overall adherence results on patient level. The adherence to the recommendations of the clinical practice guidelines showed decreased and improved levels in the LATE POST measurement compared to the EARLY POST measurement. In total, eight papers mentioned the term 'sustainability' in the conclusion (table 2) [20-26 30]. Five of these studies concluded to have sustained professionals' adherence in the late post-implementation phase [20-22 26 30], three out of eight studies described to have a 'mixed pattern', 'small desired' or 'almost' sustained professionals' adherence [23-25]. 


\section{CHAPTER 6}

Table 2. Sustainability of professionals' adherence to clinical practice guidelines

\begin{tabular}{|c|c|c|c|}
\hline Study ID & $\begin{array}{l}\text { Authors' comments in terms of sustainability of adherence } \\
\text { to the clinical practice guideline* }\end{array}$ & $\begin{array}{l}\text { Sustained } \\
\text { compared to early } \\
\text { implementation } \\
\text { results }(100 \%) \\
\text { (yes/no)** }\end{array}$ & $\begin{array}{l}\text { Sustained } \\
\text { compared to early } \\
\text { implementation } \\
\text { results }(90 \%) \\
\text { (yes/no)*** }\end{array}$ \\
\hline Ament [20] & $\begin{array}{l}\text { "Adherence to the guideline recommendations was } \\
\text { sustained in four early adopter hospitals" }\end{array}$ & yes & yes \\
\hline $\begin{array}{l}\text { Benenson } \\
{[21]}\end{array}$ & $\begin{array}{l}\text { "The observed pre pathway to post pathway differences } \\
\text { were sustained over three years" }\end{array}$ & yes & yes \\
\hline Cates [22] & $\begin{array}{l}\text { (Centre } 1 \text { \& 2) "our approach has brought about a } \\
\text { sustained reduction in the use of antibiotics for children } \\
\text { with acute otitis media, and after dissemination of our } \\
\text { findings, similar results have been replicated at centre II } \\
\text { using deferred prescribing of antibiotics for children who } \\
\text { are not unduly ill" }\end{array}$ & yes & yes \\
\hline $\begin{array}{l}\text { Enriquez- } \\
\text { Puga [23] }\end{array}$ & $\begin{array}{l}\text { "There was a small change in the desired direction in the } \\
\text { proportion of antidepressants prescribed according to } \\
\text { guidelines that lasted for } 24 \text { months, although no change } \\
\text { for antibiotics. A simple, group level educational outreach } \\
\text { intervention, designed to take account of identified } \\
\text { barriers to change, appears to have a small sustained effect } \\
\text { on prescribing levels, but the effect is not consistent across } \\
\text { different groups of drugs" }\end{array}$ & $\begin{array}{l}\text { Guideline 1: no } \\
\text { Guideline 2: yes }\end{array}$ & $\begin{array}{l}\text { Guideline 1: no } \\
\text { Guideline 2: yes }\end{array}$ \\
\hline
\end{tabular}

Forsner [24] "This study suggested that the compliance to clinical

Guideline 1: mixed Guideline 1: mixed guidelines, for treatment of depression and suicidal behaviour, was implemented and sustained over a twoyear period after an active implementation"

Higuchi [25] (1)“ The chart audit revealed that eleven nursing care indicators related to the asthma guideline recommendations showed a mixed pattern of Guideline 2: mixed Guideline 2: mixed sustainability" (2) Not mentioned

Kelly [26] "The study demonstrated a significant and sustained na change in analgesia administration practices away from the intramuscular (IM) route in favour of the IV route."

Knops [27] (1)Not mentioned

(2)Not mentioned

Guideline 1: yes Guideline 1: yes Guideline 2: no Guideline 2: no

Loszadi [28] Not mentioned yes yes

Mclaws [29] Not mentioned 
SUSTAINABILITY OF PROFESSIONALS' ADHERENCE TO GUIDELINES

\begin{tabular}{|c|c|c|c|}
\hline Study ID & $\begin{array}{l}\text { Authors' comments in terms of sustainability of adherence } \\
\text { to the clinical practice guideline* }\end{array}$ & $\begin{array}{l}\text { Sustained } \\
\text { compared to early } \\
\text { implementation } \\
\text { results }(100 \%) \\
\text { (yes/no)** }\end{array}$ & $\begin{array}{l}\text { Sustained } \\
\text { compared to early } \\
\text { implementation } \\
\text { results }(90 \%) \\
\text { (yes/no)*** }\end{array}$ \\
\hline Stephan [30] & $\begin{array}{l}\text { "One of the most important results of our intervention is } \\
\text { its sustained impact. In particular, the frequency of } \\
\text { catheter use decreased in the operating room not only } \\
\text { immediately after guideline implementation, but also could } \\
\text { be observed } 2 \text { years later." }\end{array}$ & $\begin{array}{l}\text { no } \\
\text { d }\end{array}$ & yes \\
\hline $\begin{array}{l}\text { Wakefield } \\
\text { [31] }\end{array}$ & Not mentioned & no & na \\
\hline $\begin{array}{l}\text { Williams } \\
\text { [32] }\end{array}$ & Not mentioned & yes & yes \\
\hline
\end{tabular}

* Citations of the authors of reviewed papers about the sustainability of adherence to the clinical practice guideline

** The same level or improved professionals' adherence was achieved years after implementation compared to early post-implementation results (yes/no)

***At least $90 \%$ of professionals' adherence was achieved years after implementation, compared to early post-implementation results (yes/no)

na: not applicable as the early post-implementation results were not measured; mixed: The overall professionals' adherence was not presented, and both sustained and not sustained levels of professionals' adherence to clinical practice guideline recommendations were achieved in the late post-implementation phase compared to early post-implementation results.

\section{Risk of bias in included studies}

All studies included in the present review had a high risk of bias, following the Downs and Black assessment tool [18] (table 3, additional file 5). 


\section{CHAPTER 6}

Table 3. Results of the risk of bias assessment

\begin{tabular}{|c|c|c|c|c|c|}
\hline \multirow{2}{*}{$\begin{array}{l}\text { Study ID } \\
\text { Ament [20] }\end{array}$} & \multirow{2}{*}{$\begin{array}{c}\text { Reporting } \\
\text { Unclear }\end{array}$} & \multicolumn{3}{|c|}{$\begin{array}{l}\text { External validity Internal validity - Internal validity - } \\
\qquad \text { bias confounding }\end{array}$} & Total \\
\hline & & High & High & High & High \\
\hline Benenson [21] & Unclear & High & High & High & High \\
\hline Cates [22] & High & Unclear & High & High & High \\
\hline Enriquez-Puga [23] & Unclear & High & High & High & High \\
\hline Forsner [24] & Unclear & Low & High & High & High \\
\hline Higuchi [25] & High & High & High & High & High \\
\hline Kelly [26] & High & High & High & High & High \\
\hline Knops [27] & High & Low & High & High & High \\
\hline Loszadi [28] & Unclear & High & Unclear & High & High \\
\hline Mclaws [29] & High & Low & Unclear & High & High \\
\hline Stephan [30] & High & High & Unclear & High & High \\
\hline Wakefield [31] & High & High & High & High & High \\
\hline Williams [32] & High & High & Unclear & High & High \\
\hline Total & High & High & High & High & High \\
\hline
\end{tabular}

\section{Discussion}

This systematic review identified thirteen studies, including seventeen evaluations that investigated the sustainability of professionals' adherence to a clinical practice guideline more than one year after the implementation was finished. Of seventeen analyses that focused on the extent of sustained professionals' adherence to a clinical practice guideline, seven analyses revealed fully sustained results. After decreasing the sustainability level of professionals' adherence to $90 \%$ or higher, nine out of seventeen evaluations showed sustained results. The current review showed that the number of sustainability studies is scarce and that the studies are heterogeneous with respect to their methodology. Furthermore, almost no study analysed or reflected on the updates of the guideline in the post-implementation phase. The results of this review suggest that updates of the clinical practice guidelines may have led to a warranted decrease in the adherence to the original clinical practice guideline.

As was confirmed in another systematic review [10], the sustainability studies showed to have limited methodological rigor. Two out of thirteen studies used an experimental design. The lack of identified studies in the current review suggests that most teams do not focus on the long-term performance effect of quality improvements 
[60]. Due to the limited number of studies focusing on this subject, the heterogeneity in studies, suboptimal reporting by authors and the revealed methodological weaknesses, no strong conclusions can be drawn based on the presented sustainability results. As also shown in other research, most sustainability studies used a single-case study design by focusing on a single type of programme or performed the evaluation at a single centre level [61]. The current review showed that in only two of the studies, a reference for the definition of sustainability was used. Other studies performed a sustainability evaluation without mentioning a definition. This shows the underdeveloped field of sustainability research. Also, a variety of timeframes to study the sustainability of professionals' adherence to clinical practice guidelines was revealed, varying from one and a half year to seven years following implementation.

Our review focused on the sustainability of implementation success in terms of professionals' adherence. Optimal adherence to a clinical practice guideline as determined during implementation is not always desired; for example, clinical experience and evidence may change. This systematic review included all research designs and seems to be the first review with respect to sustainability of professionals' adherence to clinical practice guidelines to date. Other reviews focused on health care from a broad perspective including multiple health care fields [10] or reviewed studies performed specifically in public health $[6,9]$. The sustainability of a health programme in public health may be influenced by other determinants than the sustainability of a clinical practice guideline in medical care. Also, the concept of the sustainability may differ between health care fields. For example, in public health sustainability of a health programme may be successfully sustained if health outcomes, e.g. changed lifestyle, are maintained and financial support is still available $[6,61]$. In medical care, the primary focus is on the quality and safety of care which is supposed to be captured in clinical practice guidelines. Due to the specific focus on clinical practice guidelines in the current review, mainly other studies were included compared to the existing sustainability reviews $[6-10,14]$.

\section{Strengths and weaknesses}

As yet, the term 'sustainability' is not consistently used for this area in the broader medical field, which presents a limitation to the electronic search strategy. The topic is not well indexed in electronic databases, and text word searches are prone to high recall and low specificity. However, it is likely that the use of a broad variety of search terms that covered sustainability, has downsized the the number of relevant studies missed and is a strength of the review.

In this systematic review, sustainability was assessed as successful if performance in terms of professionals' adherence was fully maintained in the late postimplementation phase. Also, a sensitivity analysis was performed to analyse the sustainability at a level of $90 \%$ or higher. However, as mentioned before, a limitation of the review is the high risk of bias of all studies included. The majority of the studies used a 


\section{CHAPTER 6}

retrospective data collection method. Nevertheless, results were interpreted under consideration of risk of bias, and the assessments were also used for recommendations for further research by identifying elements of studies that can be improved in new studies. Also, the question is what the best method is for evaluating sustainability. For example, retrospective data may be desired to prevent a Hawthorne effect when studying routine practice.

The results of the current review show more studies with sustained professionals' adherence than might be expected without continuing efforts and support to promote the level of sustained adherence in the post-implementation phase. Possibly, studies with unfavourable results may not be published or unsuccessful implementation projects may not be evaluated, leading to an under-representation of the true amount of work carried out in the field $[62,63]$.

\section{Implications for practice}

The current review showed that the level of the sustainability of professionals' adherence to clinical practice guidelines varies on case study level and drops in more than half of the studies. Due to the lack of sustainability research we think that sustainability failure as presented in this study is an underestimation. Unfortunately, implementation projects are primarily focused on short-term actions and short-term effect [60]. To guarantee a sustainable health care system, maintaining or improving the level of adherence to clinical practice guidelines achieved after implementation is necessary.

\section{Future research}

This review complements the existing sustainability research by focusing on sustained professionals' adherence in medical practice. The current review showed that not many studies reported data on the sustainability of professionals' adherence to clinical practice guidelines. Also, no strong conclusions can be drawn due to the high risk for bias and the heterogeneity of the studies. As shown in previous research, structural methods for sustainability evaluations are lacking $[10,64]$. More sustainability evaluation research and methodological guidance is needed to make future sustainability research more robust and generalisable and may be helpful in creating a general sustainability language.

\section{Conclusion}

This systematic review identified, reported and analysed studies that evaluated the level of sustainability of professionals' adherence to guideline recommendations in medical practice more than one year following the cessation of the implementation project. Seven out of seventeen evaluations showed sustained professionals' adherence on average 2.7 years after implementation. Due to the limited number and the lack of 
SUSTAINABILITY OF PROFESSIONALS' ADHERENCE TO GUIDELINES

methodological quality of the identified studies, no firm conclusion about the sustainability of professionals' adherence to guideline recommendations in medical practice can be drawn. More sustainability evaluations, methodological sustainability studies and reviews are needed in order to develop a general framework for sustainability measurement and to facilitate uniform language and communication within the sustainability science. 


\section{References}

1. Graham R MM, Wolman DM, Greenfield S, Steinberg E. Clinical practice guidelines we can trust. Washington (DC): National Academies Press; 2011.

2. Thomas LH, McColl E, Cullum N, Rousseau N, Soutter J, Steen N. Effect of clinical guidelines in nursing, midwifery, and the therapies: a systematic review of evaluations. Quality in Health Care. 1998 December 1, 1998;7(4):183-91.

3. Grimshaw JM, Thomas RE, MacLennan G, Fraser C, Ramsay CR, Vale L, et al. Effectiveness and efficiency of guideline dissemination and implementation strategies. Health technology assessment (Winchester, England). 2004 Feb;8(6):iii-iv, 1-72.

4. Ajzen I. The Theory of Planned Behavior. Organizational Behavior and Human Decision Processes. 1991;50(2):179-211.

5. Pluye P, Potvin L, Denis JL, Pelletier J. Program sustainability: focus on organizational routines. Health Promot Int. 2004 Dec;19(4):489-500.

6. Gruen RL, Elliott JH, Nolan ML, Lawton PD, Parkhill A, McLaren CJ, et al. Sustainability science: an integrated approach for health-programme planning. Lancet. 2008 Nov 1;372(9649):1579-89.

7. Greenhalgh T, Robert G, Macfarlane F, Bate P, Kyriakidou O. Diffusion of innovations in service organizations: systematic review and recommendations. Milbank Q. 2004;82(4):581-629.

8. Shediac-Rizkallah MC, Bone LR. Planning for the sustainability of community-based health programs: conceptual frameworks and future directions for research, practice and policy. Health Educ Res. 1998 Mar;13(1):87-108.

9. Scheirer MA. Is Sustainability Possible? A Review and Commentary on Empirical Studies of Program Sustainability. American Journal of Evaluation. 2005 September 2005;26(3):27.

10. Wiltsey Stirman S, Kimberly J, Cook N, Calloway A, Castro F, Charns M. The sustainability of new programs and innovations: a review of the empirical literature and recommendations for future research. Implementation Science. 2012;7(1):17.

11. NHS. Sustainability and its relationship with spread and adoption, General improvement skills. Coventry, UK: Improvement Leaders' guide; 2007.

12. Ament SMC, Gillissen F, Maessen JMC, Dirksen CD, van der Weijden T, von Meyenfeldt MF. Sustainability of healthcare innovations (SUSHI): long term effects of two implemented surgical care programmes (protocol). BMC health services research. 2012;12:423.

13. Adams AS, Soumerai SB, Lomas J, Ross-Degnan D. Evidence of self-report bias in assessing adherence to guidelines. International journal for quality in health care : journal of the International Society for Quality in Health Care / ISQua. 1999 Jun;11(3):187-92.

14. Buchanan D, Fitzgerald L, Ketley D, Gollop R, Jones JL, Lamont SS, et al. No going back: A review of the literature on sustaining organizational change. International Journal of Management Reviews. 2005;7(3):189-205.

15. Chambers D, Glasgow R, Stange K. The dynamic sustainability framework: addressing the paradox of sustainment amid ongoing change. Implementation Science. 2013;8(1):117.

16. Ilott I, Gerrish K, Pownall S, Eltringham S, Booth A. Exploring scale-up, spread, and sustainability: an instrumental case study tracing an innovation to enhance dysphagia care. Implementation Science. 2013;8(1):128.

17. Cochrane Effective Practice and Organisation of Care Review Group. Data Collection Checklist. University of Ottawa: Institute of Population Health; 2011.

18. Downs SH, Black N. The feasibility of creating a checklist for the assessment of the methodological quality both of randomised and non-randomised studies of health care interventions. Journal of Epidemiology and Community Health. 1998 June 1, 1998;52(6):377-84.

19. Moher D, Liberati A, Tetzlaff J, Altman DG. Preferred reporting items for systematic reviews and metaanalyses: the PRISMA statement. PLoS medicine. 2009 Jul 21;6(7):e1000097.

20. Ament SMC, Gillissen F, Maessen JMC, Dirksen CD, Bell AV, Vissers YL, et al. Sustainability of short stay after breast cancer surgery in early adopter hospitals. Breast (Edinburgh, Scotland). 2014 Mar 31. 


\section{SUSTAINABILITY OF PROFESSIONALS' ADHERENCE TO GUIDELINES}

21. Benenson R, Magalski A, Cavanaugh S, Williams E. Effects of a pneumonia clinical pathway on time to antibiotic treatment, length of stay, and mortality. Academic Emergency Medicine. 1999 December;6(12):1243-8.

22. Cates CJ. Delayed antibiotics for children with acute otitis media: Is practice change sustainable? Evidence-Based Medicine. 2009 February;14(1):2-3.

23. Enriquez-Puga A, Baker R, Paul S, Villoro-Valdes R. Effect of educational outreach on general practice prescribing of antibiotics and antidepressants: A two-year randomised controlled trialal. Scandinavian Journal of Primary Health Care. 2009;27(4):195-201.

24. Forsner T, Wistedt A, Brommels M, Janszky I, de Leon A, Forsell Y. Supported local implementation of clinical guidelines in psychiatry: a two-year follow-up. Implementation Science. 2010;5(1):4.

25. Higuchi KS, Davies BL, Edwards N, Ploeg J, Virani T. Implementation of clinical guidelines for adults with asthma and diabetes: A three-year follow-up evaluation of nursing care. Journal of Clinical Nursing. 2011 May;20(9-10):1329-38.

26. Kelly AM. Nurse-managed analgesia for renal colic pain in the emergency department. Australian health review : a publication of the Australian Hospital Association. 2000;23(2):185-9.

27. Knops AM, Storm-Versloot MN, Mank APM, Ubbink DT, Vermeulen H, Bossuyt PMM, et al. Factors influencing long-term adherence to two previously implemented hospital guidelines. International Journal for Quality in Health Care. 2010 October;22(5):421-9.

28. Lozsadi DA, Peters G, Sadik HY, Kellett MW, Fox SH, Smith DF. Prevention of osteoporosis in glucocorticoid-treated neurology patients. Clinical Neurology and Neurosurgery. 2006 February;108(2):157-62.

29. McLaws ML, Pantle AC, Fitzpatrick KR, Hughes CF. Improvements in hand hygiene across New South Wales public hospitals: clean hands save lives, part III. The Medical journal of Australia. 200919 Oct;191(8 Suppl):S18-24.

30. Stephan F, Sax H, Wachsmuth M, Hoffmeyer P, Clergue F, Pittet D. Reduction of urinary tract infection and antibiotic use after surgery: A controlled, prospective, before-after intervention study. Clinical Infectious Diseases. 200601 Jun;42(11):1544-51.

31. Wakefield B, Johnson JA, Kron-Chalupa J, Paulsen L. A research-based guideline for appropriate use of transdermal fentanyl to treat chronic pain. Oncology nursing forum. 1998 Oct;25(9):1505-13.

32. Williams A, Adams EJ, Bolderson J, Tincello DG, Richmond DH. Effect of a new guideline on outcome following third-degree perineal tears: results of a 3-year audit. International Urogynecology Journal. 2003;14(6):385-9.

33. de Kok M. Costs and effects of implementation of a short admission programme following breast cancer surgery in the Netherlands. Rotterdam: Maastricht University; 2010.

34. Ament SMC, De Kok M., Van de Velde C.J.H., Roukema J.A., Bell A.V.R.J., Van der Ent FW, Van der Weijden T., Von Meyenfeldt M.F., Dirksen C.D. Calculating implementation costs using a structured method: implementation of a short stay programme for breast cancer surgery. 2014 Submitted.

35. Mank A, Semin-Goossens A, Lelie J, Bakker P, Vos R. Monitoring hyperhydration during high-dose chemotherapy: body weight or fluid balance? Acta haematologica. 2003;109(4):163-8.

36. Storm-Versloot MN, Knops AM, Ubbink DT, Goossens A, Legemate DA, Vermeulen H. Long-term adherence to a local guideline on postoperative body temperature measurement: mixed methods analysis. J Eval Clin Pract. 2012 Aug;18(4):841-7.

37. Pantle AC, Fitzpatrick KR, McLaws ML, Hughes CF. A statewide approach to systematising hand hygiene behaviour in hospitals: clean hands save lives, part I. Med J Aust. 2009 Oct 19;191(8 Suppl):S8-S12.

38. Nationaal Borstkanker Overleg Nederland. NABON-Nota, Handboek organisatie mammazorg Amsterdam2008. Available from: http://www.oncoline.nl/uploaded/docs/mammacarcinoom/NABON\%20nota \%202008.pdf.

39. Nationaal Borstkanker Overleg Nederland. Richtlijn Mammacarcinoom, landelijke richtlijn 2012. Available from: http://www.heelkunde.nl/uploads/_a/Gk/_aGkhzjRsgiYhbhbFWFETQ/Mammacarcinoom2012.pdf.

40. Niederman MS, Bass JB, Jr., Campbell GD, Fein AM, Grossman RF, Mandell LA, et al. Guidelines for the initial management of adults with community-acquired pneumonia: diagnosis, assessment of severity, and 


\section{CHAPTER 6}

initial antimicrobial therapy. American Thoracic Society. Medical Section of the American Lung Association. The American review of respiratory disease. 1993 Nov;148(5):1418-26.

41. Niederman MS, Mandell LA, Anzueto A, Bass JB, Broughton WA, Campbell GD, et al. Guidelines for the management of adults with community-acquired pneumonia. Diagnosis, assessment of severity, antimicrobial therapy, and prevention. American journal of respiratory and critical care medicine. 2001 Jun;163(7):1730-54.

42. Mandell LA, Wunderink RG, Anzueto A, Bartlett JG, Campbell GD, Dean NC, et al. Infectious Diseases Society of America/American Thoracic Society Consensus Guidelines on the Management of CommunityAcquired Pneumonia in Adults. Clinical Infectious Diseases. 2007 March 1, 2007;44(Supplement 2):S27S72.

43. Centre for Clinical Practice at NICE (UK). Respiratory Tract Infections. Antibiotic Prescribing: Prescribing of Antibiotics for Self-Limiting Respiratory Tract Infections in Adults and Children in Primary Care: London: National Institute for Health and Clinical Excellence (UK); 2008.

44. National Institute for Health and Care Excellence (NICE). Respiratory tract infections - antibiotic prescribing: Prescribing of antibiotics for self-limiting respiratory tract infections in adults and children in primary care [September 16, 2014]. Available from: http://www.nice.org.uk/Guidance/cg69.

45. Leicestershire Health Authority. Leicestershire evidence based guidelines for the management of major depression. 2000

46. Health Protection Agency. Management of infection guidance for primary care for consultation and loca adaptation. London: HPA2005.

47. National Institue for health and care excellence (NICE). Depression in adults: The treatment and management of depression in adults. 2004.

48. Medicinskt programarbete. Regionalt vårdprogram för depressionssjukdomar inkl. mano-depressiv sjukdom. Stockholm Stockholm County Council, 2003.

49. Medicinskt programarbete. Regionalt vårdprogram. Vård av suicidnära patienter. Stockholm County Council, 2002

50. Registered Nurses' Association of Ontario, RNAO . Adult Asthma Care Best Practice Guideline. Toronto: 2002a.

51. Registered Nurses' Association of Ontario, RNAO. Reducing Foot Complications for People with Diabetes Toronto: 2004.

52. Registered Nurses' Association of Ontario, RNAO. Adult Asthma Care Guidelines for Nurses: Promoting Control of Asthma Toronto2004 [updated February 2007]. Available from: http://rnao.ca/sites/rnaoca/files/Adult_Asthma_Care_Guidelines_for_Nurses___Promoting_Control_of_Asthma.pdf.

53. Registered Nurses' Association of Ontario, RNAO. Reducing Foot Complications for people with Diabetes Toronto 2004 [updated 2007]. Available from: http://rnao.ca/sites/rnao-ca/files/Foot_Compl_Diabetes_ Updated.pdf.

54. Acute pain management guideline panel. Acute pain management: Operative or medical procedures and trauma: Clinical practice guideline. Washington DC: US Department of Health and Human Services., 1992.

55. Vermeulen H, Storm-Versloot MN, Goossens A, Speelman P, Legemate DA. Diagnostic accuracy of routine postoperative body temperature measurements. Clinical infectious diseases : an official publication of the Infectious Diseases Society of America. 2005 May 15;40(10):1404-10. Epub 2005/04/22. eng.

56. National Osteoporosis Society. Guidance on the prevention and management of corticosteroid induced osteoporosis. Bath: NOS, 1998.

57. World Health Organization, WHO. guidelines on hand hygiene in health care (advanced draft): a summary Clean hands are safer hands. Geneva: WHO, 2005.

58. Norton JA BD, Bollinger RR, et al., . Surgery: basic science and clinical evidence. 2nd ed. New York: Springer-Verlag; 2001.

59. Malouf AJ, Norton CS, Engel AF, Nicholls RJ, Kamm MA. Long-term results of overlapping anterior analsphincter repair for obstetric trauma. Lancet. 2000 Jan 22;355(9200):260-5.

60. Øvretveit J, Bate P, Cleary P, Cretin S, Gustafson D, McInnes K, et al. Quality collaboratives: lessons from research. Quality and Safety in Health Care. 2002 December 1, 2002;11(4):345-51. 


\section{SUSTAINABILITY OF PROFESSIONALS' ADHERENCE TO GUIDELINES}

61. Schell S, Luke D, Schooley M, Elliott M, Herbers S, Mueller N, et al. Public health program capacity for sustainability: a new framework. Implementation Science. 2013;8(1):15.

62. Hopewell S, Loudon K, Clarke MJ, Oxman AD, Dickersin K. Publication bias in clinical trials due to statistical significance or direction of trial results. The Cochrane database of systematic reviews. 2009 (1):MR000006.

63. Easterbrook PJ, Berlin JA, Gopalan R, Matthews DR. Publication bias in clinical research. Lancet. 1991 Apr 13;337(8746):867-72.

64. Tricco AC, Cogo E, Ashoor H, Perrier L, McKibbon KA, Grimshaw JM, et al. Sustainability of knowledge translation interventions in healthcare decision-making: protocol for a scoping review. BMJ open. 2013;3(5). 


\section{Additional file 1 \\ Electronic search strategy for MEDLINE (OvidSP)}

Database: Ovid MEDLINE <1946 to February Week 4 2014>

Date searched: 14.03 .2014

\section{Sustainability facet}

1 (adoption adj2 (longitudinal or long term or longterm)).ti,ab,ot. (64)

2 ((continued or continuation) adj2 (adherence or compliance or effect or effects or effectiveness or impact\$ or intervention\$ or innovation\$ or program\$)).ti,ab,ot. (1823)

3 (de-adoption adj2 (chang\$ or intervention\$ or innovation\$ or program\$)).ti,ab,ot. (0)

4 (diffusion adj2 (longitudinal or long term or longterm)).ti,ab,ot. (188)

5 ((discontinued or discontinuance or discontinuation) adj2 (intervention\$ or innovation\$ or program\$)).ti,ab,ot. (202)

6 ((dissemination or disseminated) adj2 (longitudinal or long term or longterm)).ti,ab,ot. (38)

7 (durability adj2 (adherence or benefit\$ or chang\$ or compliance or effect or effects or effectiveness or intervention\$ or improvement\$ or implement\$ or impact\$ or innovation\$ or longitudinal or outcome\$ or "over time" or process\$ or program\$ or post-implement\$ or success\$)).ti,ab,ot. (402)

8 (fidelity adj2 (adherence or adoption or chang\$ or compliance or effect or effects or effectiveness or evaluat\$ or impact\$ or implement\$ or improvement\$ or intervention\$ or innovation\$ or long-term or longterm or longitudinal or "over time" or outcome\$ or post-implementat\$ or program\$ or success\$)).ti,ab,ot. (605)

9 (institutionali?ation adj2 (adherence or chang\$ or compliance or effect or effects or effectiveness or improvement\$ or impact\$ or innovation\$ or longitudinal or long-term or longterm or outcome\$ or process\$ or post-implement\$)).ti,ab,ot. (235)

10 (longitudinal adj2 (adherence or assess $\$$ or benefit\$ or chang\$ or compliance or effect or effects or effectiveness or examination\$ or evaluat\$ or impact\$ or pattern? or program\$ or success\$)).ti,ab,ot. (9231)

11 ((maintenance or maintained) adj2 (adherence or chang\$ or compliance or effect or effects or effectiveness or fail\$ or intervention $\$$ or improvement $\$$ or implement $\$$ or impact or innovation $\$$ or long-term or longterm or longitudinal or outcome\$ or "over time" or process\$ or postimplement\$)).ti,ab,ot. (11874)

12 (normali?ation adj2 (adherence or chang\$ or compliance or effect or effects or effectiveness or improvement or impact\$ or innovation\$ or longitudinal or long-term or longterm or outcome\$ or process\$ or post-implement\$)).ti,ab,ot. (1069)

13 (persistence adj2 (implement\$ or innovation\$ or program\$ or long-term or longterm or "over time")).ti,ab,ot. (1551)

14 (routini\$ adj2 (chang\$ or improve\$ or intervention\$ or innovation\$ or longitudinal or long-term or longterm or outcome\$ or "over time" or program\$ or post-implement\$)).ti,ab,ot. (8)

15 (sustain\$ adj2 (adherence or adoption or assess or benefit\$ or chang\$ or compliance or evaluat\$ or effect or effects or effectiveness or fail\$ or innovation $\$$ or intervention $\$$ or improvement $\$$ or implement or impact\$ or long-term or longterm or outcome or "over time" or program\$ or post-implement\$ or success\$ or vitality)).ti,ab,ot. (15804)

16 sustainability.ti. (1367)

17 or/1-16 (43656) 


\section{SUSTAINABILITY OF PROFESSIONALS' ADHERENCE TO GUIDELINES}

\section{Guidelines facet}

18 guideline/ or practice guideline/ (24797)

19 guidelines as topic/ or practice guidelines as topic/ (108754)

20 Guideline Adherence/ (19958)

21 Health Planning Guidelines/ (3791)

22 (guideline\$ or guide-line\$).ti. (45298)

23 (practice adj3 parameter\$).ti,ab. (1081)

24 clinical protocols/ (19624)

25 guidance.ti,ab. (53787)

26 care pathway*.ti,ab. (1337)

27 critical pathway/ (4502)

28 (clinical adj3 pathway\$).ti,ab. (2907)

29 algorithms/ (168579)

30 consensus development conference.pt. (8886)

31 consensus development conference nih.pt. (725)

32 or/18-31 (396861)

3317 and 32 (1378)

\section{Animal-only study exclusion}

34 exp animals/ not (exp animals/ and humans/) (3902375)

3533 not 34 (1329) 


\section{Additional file 2}

\section{Excluded articles based on full-text selection ( $n=161$ )}

Paper

Exclusion reason

R. Adsit, D. Fraser, L. Redmond, S. Smith, and M. Fiore, 'Changing Clinical Practice, Helping No adherence People Quit: The Wisconsin Cessation Outreach Model', Wisconsin Medical Journal, 104 studied (2005), 32-36.

B. Allegranzi, A. Gayet-Ageron, N. Damani, L. Bengaly, M. L. McLaws, M. L. Moro, Z.

Timeframe $<1$ Memish, O. Urroz, H. Richet, J. Storr, L. Donaldson, and D. Pittet, 'Global Implementation of Who's Multimodal Strategy for Improvement of Hand Hygiene: A Quasi-Experimental Study', The Lancet Infectious Diseases, 13 (2013), 843-51.

J. C. Alonso, 'A Figo Project in Uruguay to Prevent Maternal Death Due to Unsafe Termination of Pregnancy', Journal of Perinatal Medicine, 41 (2013).

E. Alp, D. Haverkate, and A. Voss, 'Hand Hygiene among Laboratory Workers', Infection Control \& Hospital Epidemiology, 27 (2006), 978-80.

H. K. Amdany, and M. McMillan, 'Metronidazole Intravenous Formulation Use in inPatients in Kapkatet District Hospital, Kenya: A Best Practice Implementation Project', JBI Database of Systematic Reviews and Implementation Reports, 12 (2014), 419-32.

R. S. Bailie, D. Si, G. W. Robinson, S. J. Togni, and P. H. N. d'Abbs, 'A Multifaceted HealthService Intervention in Remote Aboriginal Communities: 3-Year Follow-up of the Impact on Diabetes Care', Medical Journal of Australia, 181 (2004), 195-200.

R. S. Bailie, S. J. Togni, D. Si, G. Robinson, and P. H. N. D'Abbs, 'Preventive Medical Care in Remote Aboriginal Communities in the Northern Territory: A Follow-up Study of the Impact of Clinical Guidelines, Computerised Recall and Reminder Systems, and Audit and Feedback', BMC Health Services Research, 3 (2003), 1-13.

Anonymous, 'Report: Cpoe Adoption a Long-Term Process', Healthcare Benchmarks \& Quality Improvement, 10 (2003), 105-7.

M. B. Goetz, T. Hoang, S. R. Henry, H. Knapp, H. D. Anaya, A. L. Gifford, and S. M. Asch, 'Evaluation of the Sustainability of an Intervention to Increase Hiv Testing', J Gen Intern Med, 24 (2009), 1275-80.

E. L. Mawdsley, S. Garcia-Houchins, and S. G. Weber, 'Back to Basics: Four Years of Sustained Improvement in Implementation of Contact Precautions at a University Hospital', Joint Commission journal on quality and patient safety / Joint Commission Resources, 36 (2010), 418-23.

R. S. Bailie, G. Robinson, S. N. Kondalsamy-Chennakesavan, S. Halpin, and Z. Wang, year

Insufficient information provided

Timeframe $<1$ year

Timeframe $<1$ year

Timeframe unclear

Timeframe unclear

No research article

No focus on a clinical practice guideline

No focus on a clinical practice guideline 'Investigating the Sustainability of Outcomes in a Chronic Disease Treatment Programme', year Soc Sci Med, 63 (2006), 1661-70.

F. E. Babl, D. Krieser, J. Belousoff, and T. Theophilos, 'Evaluation of a Paediatric Procedural Timeframe $<1$ Sedation Training and Credentialing Programme: Sustainability of Change', Emerg Med J, year 27 (2010), 577-81. 
P. A. Bampton, J. J. Sandford, and G. P. Young, 'Achieving Long-Term Compliance with $\quad$ Timeframe $<1$ Colonoscopic Surveillance Guidelines for Patients at Increased Risk of Colorectal Cancer in year Australia', International Journal of Clinical Practice, 61 (2007), 510-13.

D. Berild, T. G. Abrahamsen, S. Andresen, E. Bjorlow, O. Haug, I. M. Kossenko, O. I. Kubar, M. Lelek, S. I. Mintchenko, M. F. Pyasetskaya, S. H. Ringertz, and G. A. Sysenko, 'A

Timeframe $<1$ Controlled Intervention Study to Improve Antibiotic Use in a Russian Paediatric Hospital', International Journal of Antimicrobial Agents, 31 (2008), 478-83.

M. T. Bigham, R. Amato, P. Bondurrant, J. Fridriksson, C. D. Krawczeski, J. Raake, S.

Short term and Ryckman, S. Schwartz, J. Shaw, D. Wells, and R. J. Brilli, 'Ventilator-Associated Pneumonia in the Pediatric Intensive Care Unit: Characterizing the Problem and Implementing a Sustainable Solution', Journal of Pediatrics, 154 (2009), 582-87.e2.

long term data were presented combined

M. Fleuren, E. Dusseldorp, S. van den Bergh, H. Vlek, J. Wildschut, E. van den Akker, and D. No focus on a Wijkel, 'Implementation of a Shared Care Guideline for Back Pain: Effect on Unnecessary Referrals', Int J Qual Health Care, 22 (2010), 415-20.

K. Blanchet, and P. James, 'Can International Health Programmes Be Sustained after the End of International Funding: The Case of Eye Care Interventions in Ghana', BMC Health Serv Res, 14 (2014), 77

P. G. Teixeira, K. Inaba, J. Dubose, N. Melo, M. Bass, H. Belzberg, and D. Demetriades, 'Measurable Outcomes of Quality Improvement Using a Daily Quality Rounds Checklist: Two-Year Prospective Analysis of Sustainability in a Surgical Intensive Care Unit', The Journal of Trauma and Acute Care Surgery, 75 (2013), 717-21.

L. Bowers, C. Berry-Caban, and R. Munson, 'Implementing Process Improvement Efforts to Increase Adherence with Diabetes Clinical Practice Guidelines in a Military Treatment Facility', Journal of the American Pharmacists Association, 52 (5) (2012), 671.

C. C. Bowman, E. J. Sobo, S. M. Asch, and A. L. Gifford, 'Measuring Persistence of Implementation: Queri Series', Implement Sci, 3 (2008), 21.

C. Brand, F. Landgren, A. Hutchinson, C. Jones, L. MacGregor, and D. Campbell, 'Clinical Practice Guidelines: Barriers to Durability after Effective Early Implementation', Interna Medicine Journal, 35 (2005), 162-69.

B. D. Brazzell, 'Improving High Hand-Hygiene Compliance and Reducing Healthcareassociated Infection in Eight Nursing Units', American Journal of Infection Control, 1) (2014), S25-S26

A. S. Brent, W. M. Rahman, L. L. Knarr, J. A. Harrison, K. L. Kearns, and D. S. Lindstrom, 'Reducing Cycle Times in Pediatric Emergency Medicine', Pediatric Emergency Care, 25 (2009), 307-11.

D. L. Whitford, S. H. Roberts, and S. Griffin, 'Sustainability and Effectiveness of clinical practice guideline

No focus on a clinical practice guideline

No focus on a clinical practice guideline

Insufficient information provided

No research article One measurement

Timeframe $<1$ year

No adherence studied

Timeframe unclear . Diabet Med, 21 (2004), 1221

No research article 
Ali A. Cheema, Annette M. Scott, Karen J. Shambaugh, Jacqueline N. Shaffer-Hartman, Short term and Ronald E. Dechert, Susan M. Hieber, John W. Gosbee, and Matthew F. Niedner, 'Rebound long term data in Ventilator-Associated Pneumonia Rates During a Prevention Checklist Washout Period', were presented BMJ Quality \& Safety, 20 (2011), 811-17. combined

Y. C. Chen, W. H. Sheng, J. T. Wang, S. C. Chang, H. C. Lin, K. L. Tien, L. Y. Hsu, and K. S. Tsai, Timeframe $<1$ 'Effectiveness and Limitations of Hand Hygiene Promotion on Decreasing Healthcare- year Associated Infections', PLoS ONE [Electronic Resource], 6 (2011), e27163.

L. L. Chlan, J. L. Guttormson, and K. Savik, 'Tailoring a Treatment Fidelity Framework for an Timeframe $<1$ Intensive Care Unit Clinical Trial', Nursing Research, 60 (2011), 348-53.

year

B. Crawford, M. Skeath, and A. Whippy, 'Kaiser Permanente Northern California Sepsis Mortality Reduction Initiative', Critical Care, 16 (2012).

Timeframe $<1$ year

J. D. Crews, E. Whaley, D. Syblik, and J. Starke, 'Sustained Improvement in Hand Hygiene at Timeframe $<1$ a Children's Hospital', Infection Control and Hospital Epidemiology, 34 (2013), 751-53. $\quad$ year

S. B. DeMauro, E. Douglas, K. Karp, B. Schmidt, J. Patel, A. Kronberger, R. Scarboro, and M. Short term and Posencheg, 'Improving Delivery Room Management for Very Preterm Infants', Pediatrics, long term data 132 (2013), e1018-e25.

S. Deuster, I. Roten, and S. Muehlebach, 'Implementation of Treatment Guidelines to Support Judicious Use of Antibiotic Therapy', Journal of Clinical Pharmacy and Therapeutics, 35 (2010), 71-78.

M. A. Doyle, S. Brez, S. Sicoli, F. De Sousa, E. Keely, and J. C. Malcom, 'Using Standardized Insulin Orders to Improve Patient Safety in a Tertiary Care Centre', Canadian Journal of Diabetes, 38 (2014), 118-25.

K. Ellingson, R. R. Muder, R. Jain, D. Kleinbaum, P. J. Feng, C. Cunningham, C. Squier, J. Lloyd, J. Edwards, V. Gebski, and J. Jernigan, 'Sustained Reduction in the Clinical Incidence of Methicillin-Resistant Staphylococcus Aureus Colonization or Infection Associated with a Multifaceted Infection Control Intervention', Infect Control Hosp Epidemiol, 32 (2011), 18.

J. N. Epstein, J. M. Langberg, P. K. Lichtenstein, R. C. Kolb, and L. J. Stark, 'Sustained Improvement in Pediatricians' Adhd Practice Behaviors in the Context of a CommunityBased Quality Improvement Initiative', Children's Health Care, 39 (2010), 296-311.

K. J. Evans, J. Thompson, S. E. Spratt, L. F. Lien, and A. Vorderstrasse, 'The Implementation and Evaluation of an Evidence-Based Protocol to Treat Diabetic Ketoacidosis: A Quality Improvement Study', Adv Emerg Nurs J, 36 (2014), 189-98.

M. Fakhry, G. B. Hanna, O. Anderson, A. Holmes, and D. Nathwani, 'Effectiveness of an were presented combined

Timeframe $<1$ year

Insufficient information provided

No adherence studied

Timeframe $<1$ year Timeframe $<1$ year Audible Reminder on Hand Hygiene Adherence', American Journal of Infection Control, 40 (2012), 320-23.

M. G. Fakih, K. Jones, J. E. Rey, R. Takla, S. Szpunar, K. Brown, A. Boelstler, and L. Saravolatz, 'Peripheral Venous Catheter Care in the Emergency Department: Education and Feedback Lead to Marked Improvements', American Journal of Infection Control, 41 (2013), 531-6.

Timeframe $<1$

Timeframe $<1$ year year 
M. Bunik, M. J. Federico, B. Beaty, M. Rannie, J. T. Olin, and A. Kempe, 'Quality Timeframe $<1$ Improvement for Asthma Care within a Hospital-Based Teaching Clinic', Academic year Pediatrics, 11 (2011), 58-65.

B. R. Ferrell, and R. Virani, 'Institutional Commitment to Improved Pain Management: Sustaining the Effort', Journal of Pharmaceutical Care in Pain and Symptom Control, 6 (1998), 43-55

G. T. Feyissa, J. S. Gomersall, and S. Robertson-Malt, 'Compliance to Hand Hygiene Practice Timeframe $<1$ among Nurses in Jimma University Specialized Hospital in Ethiopia: A Best Practice year Implementation Project', JBI Database of Systematic Reviews and Implementation Reports, 12 (2014), 318-37.

A. M. Finucane, B. Stevenson, R. Moyes, D. Oxenham, and S. A. Murray, 'Improving End-of- Timeframe $<1$ Life Care in Nursing Homes: Implementation and Evaluation of an Intervention to Sustain year Quality of Care', Palliative Medicine, 27 (2013), 772-78.

A. Fisher, 'How Nhs Trusts Are Tackling Healthcare-Associated Infections', Nursing management (Harrow, London, England : 1994), 16 (2010), 26-27.

No research article

E. J. Gallagher, and S. W. Trotzky, 'Sustained Effect of an Intervention to Limit Ordering of No focus on a Emergency Department Lumbosacral Spine Films', Journal of Emergency Medicine, 16 clinical practice (1998), 395-401.

guideline

G. C. Fonarow, M. J. Reeves, E. E. Smith, J. L. Saver, X. Zhao, D. W. Olson, A. F. Hernandez, No focus on a E. D. Peterson, L. H. Schwamm, G. WTG-Stroke Steering Committee, and Investigators, clinical practice 'Characteristics, Performance Measures, and in-Hospital Outcomes of the First One Million guideline Stroke and Transient Ischemic Attack Admissions in Get with the Guidelines-Stroke', Circulation. Cardiovascular Quality \& Outcomes, 3 (2010), 291-302.

D. Fonda, J. Cook, V. Sandler, and M. Bailey, 'Sustained Reduction in Serious Fall-Related Injuries in Older People in Hospital', Medical Journal of Australia, 184 (2006), 379-82.

S. P. Ford, M. K. Leick-Rude, K. A. Meinert, B. Anderson, M. B. Sheehan, B. M. Haney, S. R. No adherence Leeks, S. D. Simon, and J. K. Jackson, 'Overcoming Barriers to Oxygen Saturation Targeting', studied Pediatrics, 118 Suppl 2 (2006), S177-86.

J. Fortney, M. Enderle, S. McDougall, J. Clothier, J. Otero, L. Altman, and G. Curran, Timeframe $<1$ 'Implementation Outcomes of Evidence-Based Quality Improvement for Depression in Va year Community Based Outpatient Clinics', Implement Sci, 7 (2012), 30.

J. B. Froehlich, D. Karavite, P. L. Russman, N. Erdem, C. Wise, G. Zelenock, T. Wakefield, J. $\quad$ No adherence Stanley, K. A. Eagle, Cardiology American College of, and Association American Heart, 'American College of Cardiology/American Heart Association Preoperative Assessment Guidelines Reduce Resource Utilization before Aortic Surgery', Journal of Vascular Surgery, 36 (2002), 758-63.

L. Y. Fu, M. Weissman, R. McLaren, C. Thomas, J. Campbell, J. Mbafor, U. Doshi, and D. $\quad$ Timeframe $<1$ Cora-Bramble, 'Improving the Quality of Immunization Delivery to an at-Risk Population: A year Comprehensive Approach', Pediatrics, 129 (2012), e496-e503. 
C. Fuller, S. Michie, J. Savage, J. McAteer, S. Besser, A. Charlett, A. Hayward, B. D. Cookson, Timeframe $<1$ B. S. Cooper, G. Duckworth, A. Jeanes, J. Roberts, L. Teare, and S. Stone, 'The Feedback year Intervention Trial (Fit) - Improving Hand-Hygiene Compliance in Uk Healthcare Workers: A Stepped Wedge Cluster Randomised Controlled Trial', PLoS ONE, 7 (2012).

P. W. Lee, A. J. Dietrich, T. E. Oxman, J. W. Williams, Jr., and S. L. Barry, 'Sustainable Impact No focus on a of a Primary Care Depression Intervention', J Am Board Fam Med, 20 (2007), 427-33. clinical practice guideline

V. Ganju, 'Mental Health Quality and Accountability: The Role of Evidence-Based Practices No research and Performance Measurement', Administration \& Policy in Mental Health, 33 (2006), article 659-65.

M. Gazarian, and L. V. Graudins, 'Long-Term Reduction in Adverse Drug Events: An Evidence-Based Improvement Model', Pediatrics, 129 (2012), e1334-e42.

K. M. Gendron, S. Y. Lai, G. S. Weinstein, A. A. Chalian, J. M. Husbands, P. F. Wolf, L. DiDonato, and R. S. Weber, 'Clinical Care Pathway for Head and Neck Cancer: A Valuable Tool for Decreasing Resource Utilization', Archives of Otolaryngology - Head and Neck Surgery, 128 (2002), 258-62.

M. F. Gerdtz, R. Waite, T. Vassiliou, B. Garbutt, R. Prematunga, and E. Virtue, 'Evaluation of Timeframe $<1$ a Multifaceted Intervention on Documentation of Vital Signs at Triage: A before-and-after year Study', EMA - Emergency Medicine Australasia, 25 (2013), 580-87.

V. Gibbons, T. Esselink, and S. McHugh, 'Assessing Practice Relating to Fall Risk Insufficient Management among Nurses in an Acute Ward Setting: A Best Practice Implementation information Report', JBI Database of Systematic Reviews and Implementation Reports, 11 (2013), 275- provided 85.

F. Gillissen, S. M. C. Ament, J. M. C. Maessen, C. D. Dirksen, T. Van Der Weijden, and M. F. Timeframe $<1$ Von Meyenfeldt, 'Sustainability of an Enhanced Recovery Programme in Colonic Surgery in year the Netherlands', European Journal of Cancer, 49 (2013), S533-S34.

Edward M. Glaser, 'Durability of Innovations in Human Service Organizations: A Case-Study No focus on a Analysis', Science Communication, 3 (1981), 167-85. clinical practice guideline

S. G. Anthony, L. M. Prevedello, M. M. Damiano, T. K. Gandhi, P. M. Doubilet, S. E. Seltzer, and R. Khorasani, 'Impact of a 4-Year Quality Improvement Initiative to Improve Communication of Critical Imaging Test Results', Radiology, 259 (2011), 802-07.

L. Gonzalez, J. F. Elgart, H. Calvo, and J. J. Gagliardino, 'Changes in Quality of Care and Costs Induced by Implementation of a Diabetes Program in a Social Security Entity of Argentina', ClinicoEconomics and Outcomes Research, 5 (2013), 337-45.

M. L. Grayson, P. L. Russo, M. Cruickshank, J. L. Bear, C. A. Gee, C. F. Hughes, P. D. R. Johnson, R. McCann, A. J. McMillan, B. G. Mitchell, C. E. Selvey, R. E. Smith, and I. J. Wilkinson, 'Outcomes from the First 2 Years of the Australian National Hand Hygiene Initiative', Medical Journal of Australia, 195 (2011), 615-19.

T. Greenhalgh, F. Macfarlane, C. Barton-Sweeney, and F. Woodard, '"If We Build It, Will It No adherence Stay?" A Case Study of the Sustainability of Whole-System Change in London', Milbank Q, studied 90 (2012), 516-47. 
SUSTAINABILITY OF PROFESSIONALS' ADHERENCE TO GUIDELINES

Paper

Exclusion reason

R. Grol, J. Braspenning, R. Dijkstra, M. Hulscher, and M. Wensing, 'Implementation of Nhg No research Guidelines: Success or Problem?. [Dutch]

article

Terry J. Hannan, Stanley Bart, Colin Sharp, Mathew J. Fassett, and Robert G. Fassett, 'The Sustainability of Medical Morning Handover Reporting: Adherence in a Regional Hospital', Australian Health Review, 34 (2010), 325-27.

M. F. Harris, C. Hobbs, G. P. Davies, S. Simpson, D. Bernard, and A. Stubbs,

No focus on a

clinical practice guideline

'Implementation of a Snap Intervention in Two Divisions of General Practice: A Feasibility

Timeframe $<1$ Study', Medical Journal of Australia, 183 (2005), S54-S58.

J. S. Haukoos, E. Hopkins, V. T. Eliopoulos, R. L. Byyny, K. A. Laperriere, M. X. Mendoza, M. No adherence W. Thrun, and H. I. V. Testing Study Group Denver Emergency Department Rapid, Studied 'Development and Implementation of a Model to Improve Identification of Patients Infected with Hiv Using Diagnostic Rapid Testing in the Emergency Department', Academic Emergency Medicine, 14 (2007), 1149-57.

D. Henry, F. R. Muriel, and P. Hirway, 'Sustaining Improvement in Surgical Infection Timeframe $<1$ Prevention Measures for Hysterectomy', Journal for healthcare quality : official publication year of the National Association for Healthcare Quality, 29 (2007), 50-56.

Kathryn Smith Higuchi, Angela Downey, Barbara Davies, Irmajean Bajnok, and Melissa Waggott, 'Using the Nhs Sustainability Framework to Understand the Activities and Resource Implications of Canadian Nursing Guideline Early Adopters', Journal of Clinical Nursing, 22 (2013), 1707-16.

P. B. Howard, P. El-Mallakh, A. L. Miller, M. K. Rayens, G. R. Bond, K. Henderson, and A. T. Cooley, 'Prescriber Fidelity to a Medication Management Evidence-Based Practice in the Treatment of Schizophrenia', Psychiatric Services, 60 (2009), 929-35.

P. Hudson, and A. Kupa, 'Anaphylaxis Discharge Management "Reducing the Risk"', Internal Medicine Journal, 40 (2010), 12.

- - - 'Optimising Anaphylaxis Discharge Management', Allergy: European Journal of Allergy and Clinical Immunology, 65 (2010), 44.

No adherence studied

Paper is not about sustainability Insufficient information provided

Insufficient information provided

A. Jamal, G. O'Grady, E. Harnett, D. Dalton, and D. Andresen, 'Improving Hand Hygiene in a Timeframe $<1$ Paediatric Hospital: A Multimodal Quality Improvement Approach', BMJ Quality \& Safety, year 21 (2012), 171-76.

K. Johnson, and A. Grossman, 'Implementation and Maintenance of Practice Guidelines to Decrease Central Line Associated Bloodstream Infections by Minimizing Line

Insufficient Manipulation', Biology of Blood and Marrow Transplantation, 1) (2013), S172.

information provided

R. Johnstone, A. Jones, and A. Fowell, 'Welsh Collaborative Care Pathway Project; 10 Years No adherence Experience of Implementing and Maintaining a Care Pathway for the Last Days of Life', $\quad$ studied International Journal of Care Pathways, 15 (2011), 39-43.

L. C. Kahwati, T. X. Lance, K. R. Jones, and L. S. Kinsinger, 'Re-Aim Evaluation of the Veterans Health Administration's Move! Weight Management Program', Translationa Behavioral Medicine, 1 (2011), 551-60.

Paper is not

about

sustainability 
T. G. Kampmeier, R. P. Lukas, C. Steffler, C. Sauerland, T. P. Weber, H. Van Aken, and A. Bohn, 'Chest Compression Depth after Change in Cpr Guidelines-Improved but Not Timeframe $<1$ Sufficient', Resuscitation, 85 (2014), 503-08.

L. S. Kao, D. F. Lew, P. D. Doyle, M. M. Carrick, V. S. Jordan, E. J. Thomas, and K. P. Lally, 'A Tale of 2 Hospitals: A Staggered Cohort Study of Targeted Interventions to Improve Compliance with Antibiotic Prophylaxis Guidelines', Surgery, 148 (2010), 255-62.

S. M. Kellie, A. Timmins, and C. Brown, 'A Statewide Collaborative to Reduce MethicillinResistant Staphylococcus Aureus Bacteremias in New Mexico', Joint Commission journal on quality and patient safety / Joint Commission Resources, 37 (2011), 154-62.

D. J. Kenny, and P. Goodman, 'Care of the Patient with Enteral Tube Feeding: An EvidenceBased Practice Protocol', Nursing Research, 59 (2010), S22-S31.

J. King, and J. A. Gold, 'Sustaining Improvements in Quality', Wisconsin Medical Journal, 113 (2014), 37.

R. Knippenberg, A. Soucat, K. Oyegbite, M. Sene, D. Broun, K. Pangu, I. Hopwood, R. Grandcourt, K. L. Tinguiri, I. Fall, S. Ammassari, and E. Alihonou, 'Sustainability of Primary Health Care Including Expanded Program of Immunizations in Bamako Initiative Programs in West Africa: An Assessment of 5 Years' Field Experience in Benin and Guinea', International Journal of Health Planning and Management, 12 (1997), S9-S28.

J. D. Knudsen, and S. E. Andersen, 'A Multidisciplinary Intervention to Reduce Infections of Timeframe $<1$ Esbl- and Ampc-Producing, Gram-Negative Bacteria at a University Hospital', PLoS ONE, 9 year (2014).

B. S. Kodali, D. Kim, R. Bleday, H. Flanagan, and R. D. Urman, 'Successful Strategies for the Reduction of Operating Room Turnover Times in a Tertiary Care Academic Medical Center', Journal of Surgical Research, 187 (2014), 403-11.

D. J. Kolko, A. M. Iselin, and K. J. Gully, 'Evaluation of the Sustainability and Clinical Outcome of Alternatives for Families: A Cognitive-Behavioral Therapy (Af-Cbt) in a Child Protection Center', Child Abuse Negl, 35 (2011), 105-16.

R. Kramer, R. Groom, D. Weldner, P. Gallant, B. Heyl, R. Knapp, and A. Arnold, 'Glycemic Control and Reduction of Deep Sternal Wound Infection Rates: A Multidisciplinary Approach', Archives of Surgery, 143 (2008), 451-6.

J. Lagan, P. Garg, J. M. Tang, and M. Burgess, 'Oxygen Therapy in Patients with Chest Pain of Acute Onset: Single Centre Audit Experience', British Journal of Hospital Medicine, 74 (2013), 347-9.

B. Lai, C. Gibb, J. Pink, and L. Thomas, 'Sustainability of a Pharmacist-Driven Pathway for Osteoporosis-Related Fractures on an Orthopaedic Unit after a 5-Year Period', International Journal of Pharmacy Practice, 20 (2012), 134-37.

B. C. C. Lam, J. Lee, and Y. L. Lau, 'Hand Hygiene Practices in a Neonatal Intensive Care Unit: A Multimodal Intervention and Impact on Nosocomial Infection', Pediatrics, 114 (2004), e565-71.

S. M. Levy, C. E. Senter, R. B. Hawkins, J. Y. Zhao, K. Doody, L. S. Kao, K. P. Lally, and K. No focus on a clinical practice guideline

One measurement

No adherence studied

Timeframe unclear

Timeframe $<1$ year

Timeframe $<1$ year Tsao, 'Implementing a Surgical Checklist: More Than Checking a Box', Surgery, 152 (2012), 331-6. 
S. L. Lim, S. C. Ng, J. Lye, W. C. Loke, M. Ferguson, and L. Daniels, 'Improving the Timeframe $<1$ Performance of Nutrition Screening through a Series of Quality Improvement Initiatives', year Joint Commission Journal on Quality \& Patient Safety, 40 (2014), 178-86.

N. Lubisch, R. Roskos, and S. M. Sattler, 'Improving Outcomes in Pediatric Procedural Sedation', Joint Commission Journal on Quality \& Patient Safety, 34 (2008), 192-5.

S. Mace, and D. Taylor, 'Improving Adherence to Nice Guidance for Bipolar Illness: Valproate Use in Women of Childbearing Potential', Psychiatrist, 35 (2011), 63-67.

R. MacRedmond, K. Hollohan, R. Stenstrom, R. Nebre, D. Jaswal, and P. Dodek, 'Introduction of a Comprehensive Management Protocol for Severe Sepsis Is Associated with Sustained Improvements in Timeliness of Care and Survival', Quality \& Safety in Health Care, 19 (2010), e46.

A. Maher, 'A Multi-Faceted Approach to Reducing Risk of Bloodstream Infections for Pediatric Rehabilitation Patients Receiving Total Parenteral Nutrition', American Journal of year Infection Control, 37 (5) (2009), E37-E38.

G. P. Martin, S. Weaver, G. Currie, R. Finn, and R. McDonald, 'Innovation Sustainability in Challenging Health-Care Contexts: Embedding Clinically Led Change in Routine Practice', Health Serv Manage Res, 25 (2012), 190-9.

B. C. Marshall, and E. C. Nelson, 'Accelerating Implementation of Biomedical Research Advances: Critical Elements of a Successful 10 Year Cystic Fibrosis Foundation Healthcare Delivery Improvement Initiative', BMJ Quality and Safety, 23 (2014), i95-i103.

Mohamad G. Fakih, Janice E. Rey, Margarita E. Pena, Susanna Szpunar, and Louis D. Saravolatz, 'Sustained Reductions in Urinary Catheter Use over 5 Years: Bedside Nurses View Themselves Responsible for Evaluation of Catheter Necessity', American Journal of Infection Control, 41 (2013), 236-39.

J. Mayer, B. Mooney, A. Gundlapalli, S. Harbarth, G. J. Stoddard, M. A. Rubin, L. Eutropius, B. Brinton, and M. H. Samore, 'Dissemination and Sustainability of a Hospital-Wide Hand Hygiene Program Emphasizing Positive Reinforcement', Infection Control \& Hospital Epidemiology, 32 (2011), 59-66.

W. M. McClellan, D. L. Frankenfield, P. R. Frederick, W. D. Flanders, A. Alfaro- Correa, M Rocco, and S. D. Helgerson, 'Can Dialysis Therapy Be Improved? A Report from the Esrd Core Indicators Project', American Journal of Kidney Diseases, 34 (1999), 1075-82.

R. McDermott, F. Tulip, B. Schmidt, and A. Sinha, 'Sustaining Better Diabetes Care in Remote Indigenous Australian Communities', British Medical Journal, 327 (2003), 428-30.

C. McDonnell, 'Interventions Guided by Analysis of Quality Indicators Decrease the Frequency of Laryngospasm During Pediatric Anesthesia', Paediatric Anaesthesia, 23 (2013), 579-87.

M. McDonnell, C. Reynolds, E. Nic Dhonncha, A. O'Connell, M. O'Donnell, and T. Walsh, 'Audit of Acute Stroke Care: From the Emergency Department to the Acute Stroke Unit', Irish Journal of Medical Science, 181 (2012), S277. Timeframe $<1$

Insufficient information provided

Timeframe $<1$ year

Timeframe $<1$ year

No adherence studied

No research article No adherence studied

Timeframe $<1$ year

Timeframe $<1$ year

No adherence studied

Timeframe $<1$ year

Insufficient information provided 
G. J. McHugo, R. E. Drake, R. Whitley, G. R. Bond, K. Campbell, C. A. Rapp, H. H. Goldman, W. J. Lutz, and M. T. Finnerty, 'Fidelity Outcomes in the National Implementing EvidenceBased Practices Project', Psychiatric Services, 58 (2007), 1279-84.

Christine McMullan, Grace Propper, Christine Schuhmacher, Lisa Sokoloff, David Harris, Paul Murphy, and William H. Greene, 'A Multidisciplinary Approach to Reduce Central Line-Associated Bloodstream Infections', Joint Commission Journal on Quality \& Patient Safety, 39 (2013), 61-69.

K. Meier, 'Sustainability in Health Care - Changing Mind, Knowledge and Behaviour', European Journal of Oncology Pharmacy, 6 (2012), 3.

M. J. Mello, J. Bromberg, J. Baird, T. Nirenberg, T. Chun, C. Lee, and J. G. Linakis, Paper is not about sustainability Timeframe $<1$ year 'Translation of Alcohol Screening and Brief Intervention Guidelines to Pediatric Trauma Centers', Journal of Trauma and Acute Care Surgery, 75 (2013), S301-S07.

J. Melnikow, N. D. Kohatsu, and B. K. S. Chan, 'Put Prevention into Practice: A Controlled Evaluation', American Journal of Public Health, 90 (2000), 1622-25.

W. C. Mertens, D. J. Higby, D. Brown, R. Parisi, J. Fitzgerald, E. M. Benjamin, and P. K. Lindenauer, 'Improving the Care of Patients with Regard to Chemotherapy-Induced Nausea and Emesis: The Effect of Feedback to Clinicians on Adherence to Antiemetic Prescribing Guidelines', Journal of Clinical Oncology, 21 (2003), 1373-78.

A. Mian, C. Russell, M. Honeycutt, and C. Oldridge, 'Multidisciplinary Approach to Reduction of Central Line Associated Blood Stream Infections (Clabsi): An Institutional Experience with Collaborative Guidelines', The Journal of the Arkansas Medical Society, 109 (2012), 128-31

M. R. Miller, M. Griswold, J. M. Harris, II, G. Yenokyan, W. C. Huskins, M. Moss, T. B. Rice, Timeframe $<1$ D. Ridling, D. Campbell, P. Margolis, S. Muething, and R. J. Brilli, 'Decreasing Picu Catheter- year Associated Bloodstream Infections: Nachri's Quality Transformation Efforts.... National Association of Children's Hospitals and Related Institutions', Pediatrics, 125 (2010), 20613

M. R. Miller, M. F. Niedner, W. C. Huskins, E. Colantuoni, G. Yenokyan, M. Moss, T. B. Rice, D. Ridling, D. Campbell, and R. J. Brilli, 'Reducing Picu Central Line - Associated Bloodstream Infections: 3-Year Results', Pediatrics, 128 (2011), e1077-e83.

L. S. Segre, M. W. O'Hara, R. L. Brock, and D. Taylor, 'Depression Screening of Perinatal Women by the Des Moines Healthy Start Project: Program Description and Evaluation', Psychiatric Services, 63 (2012), 250-5.

No research article Timeframe $<1$ year

Timeframe $<1$ year

Timeframe $<1$ year

Insufficient information provided D. M. Nestler, A. Noheria, L. H. Haro, L. G. Stead, W. W. Decker, L. N. Scanlan-Hanson, R. J. No adherence Lennon, C. C. Lim, D. R. Holmes, C. S. Rihal, M. R. Bell, and H. H. Ting, 'Sustaining studied Improvement in Door-to-Balloon Time over 4 Years: The Mayo Clinic St-Elevation Myocardial Infarction Protocol', Circulation: Cardiovascular Quality and Outcomes, 2 (2009), 508-13. 
G. T. O'Connor, S. K. Plume, E. M. Olmstead, J. R. Morton, C. T. Maloney, W. C. Nugent, F. No focus on a Hernandez, Jr., R. Clough, B. J. Leavitt, L. H. Coffin, C. A. Marrin, D. Wennberg, J. D. clinical practice Birkmeyer, D. C. Charlesworth, D. J. Malenka, H. B. Quinton, and J. F. Kasper, 'A Regional guideline Intervention to Improve the Hospital Mortality Associated with Coronary Artery Bypass Graft Surgery. The Northern New England Cardiovascular Disease Study Group', JAMA, 275 (1996), 841-6.

B. Ostrowsky, S. Sharma, M. Defino, Y. Guo, P. Shah, S. McAllen, P. Chung, S. Brown, J. Paternoster, A. Schechter, B. Yongue, and R. Bhalla, 'Antimicrobial Stewardship and Automated Pharmacy Technology Improve Antibiotic Appropriateness for CommunityAcquired Pneumonia', Infection Control and Hospital Epidemiology, 34 (2013), 566-72.

E. Pawlowski, and D. Hobbs, 'A Large Community Hospital's Multi-Disciplinary Approach to Code Stroke', Stroke, 44 (12) (2013), e203.

Timeframe $<1$ year

Insufficient information provided

P. H. Perlstein, U. R. Kotagal, P. J. Schoettker, H. D. Atherton, M. K. Farrell, W. E. Gerhardt, and M. P. Alfaro, 'Sustaining the Implementation of an Evidence-Based Guideline for Bronchiolitis', in Archives of Pediatrics \& Adolescent Medicine (2000), pp. 1001-07.

D. Petroudi, 'Nosocomial Infections and Staff Hygiene', Journal of Infection in Developing Countries, 3 (2009), 152-56.

J. A. Al-Tawfiq, M. S. Abed, N. Al-Yami, and R. B. Birrer, 'Promoting and Sustaining a Hospital-Wide, Multifaceted Hand Hygiene Program Resulted in Significant Reduction in Health Care-Associated Infections', American Journal of Infection Control, 41 (2013), 482 6.

A. J. Porter, J. Y. Narimasu, M. F. Mulroy, and R. P. Koehler, 'Sustainable, Effective Implementation of a Surgical Preprocedural Checklist: An "Attestation" Format for All Operating Team Members', Joint Commission journal on quality and patient safety / Joint Commission Resources, 40 (2014), 3-9.

P. J. Pronovost, C. A. Goeschel, E. Colantuoni, S. Watson, L. H. Lubomski, S. M. Berenholtz, D. A. Thompson, D. J. Sinopoli, S. Cosgrove, J. B. Sexton, J. A. Marsteller, R. C. Hyzy, R. Welsh, P. Posa, K. Schumacher, and D. Needham, 'Sustaining Reductions in Catheter Related Bloodstream Infections in Michigan Intensive Care Units: Observational Study', BMJ, 340 (2010), c309.

S. Provost, R. Pineault, P. Tousignant, M. Hamel, and R. B. Da Silva, 'Evaluation of the Implementation of an Integrated Primary Care Network for Prevention and Management of Cardiometabolic Risk in Montreal', BMC family practice, 12 (2011), 126.

B. R. Rachman, and R. B. Mink, 'A Prospective Observational Quality Improvement Study of the Sustained Effects of a Program to Reduce Unplanned Extubations in a Pediatric Intensive Care Unit', Paediatric Anaesthesia, 23 (2013), 614-20.

H. Ragazzi, A. Keller, R. Ehrensberger, and A. M. Irani, 'Evaluation of a Practice-Based Timeframe $<1$ year

No research article

Timeframe $<1$ year

Timeframe $<1$ year

No adherence studied

No research article No adherence studied Intervention to Improve the Management of Pediatric Asthma', Journal of urban health : bulletin of the New York Academy of Medicine, 88 Suppl 1 (2011), 38-48.

C. A. Rapp, R. J. Goscha, and L. S. Carlson, 'Evidence-Based Practice Implementation in Kansas', Community mental health journal, 46 (2010), 461-65.

No adherence studied 
A. Rawn, and K. Wilson, 'Standardized Network Order Sets in Rural Ontario: A Follow-up

G. Regev-Yochay, M. Raz, R. Dagan, H. Roizin, B. Morag, S. Hetman, S. Ringel, N. Ben-Israel, Timeframe $<1$ M. Varon, E. Somekh, and E. Rubinstein, 'Reduction in Antibiotic Use Following a Cluster year Randomized Controlled Multifaceted Intervention: The Israeli Judicious Antibiotic Prescription Study', Clinical Infectious Diseases, 53 (2011), 33-41.

S. Reid, and J. McFarlin, 'Environmental Hygiene Sustainability-Is It Possible?', American Journal of Infection Control, 40 (5) (2012), e68-e69.

Insufficient information provided

M. J. Richards, M. B. Robertson, J. G. A. Dartnell, M. M. Duarte, N. R. Jones, D. A. Kerr, L. L. No focus on a Lim, P. D. Ritchie, G. J. Stanton, and S. E. Taylor, 'Impact of a Web-Based Antimicrobial Approval System on Broad-Spectrum Cephalosporin Use at a Teaching Hospital', Medical Journal of Australia, 178 (2003), 386-90.

S. A. Roberts, C. Sieczkowski, T. Campbell, G. Balla, A. Keenan, Steering Auckland District Health Board Hand Hygiene, and Groups Working, 'Implementing and Sustaining a Hand Hygiene Culture Change Programme at Auckland District Health Board', New Zealand Medical Journal, 125 (2012), 75-85.

M. T. Roe, 'Success Stories: How Hospitals Are Improving Care', American Heart Journal, 148 (2004), S52-S55.

J. M. Rothschild, S. McGurk, M. Honour, A. A. McClendon, P. Srivastava, W. H. Churchill, R. M. Kaufman, J. Avorn, E. F. Cook, and D. W. Bates, 'Assessment of Education and Computerized Decision Support Interventions for Improving Transfusion Practice', Transfusion, 47 (2007), 228-39.

D. Bird, A. Zambuto, C. O'Donnell, J. Silva, C. Korn, R. Burke, P. Burke, and S. Agarwal, 'Adherence to Ventilator-Associated Pneumonia Bundle and Incidence of VentilatorAssociated Pneumonia in the Surgical Intensive Care Unit', Archives of Surgery, 145 (2010), 465-70.

F. H. Rubin, K. Neal, K. Fenlon, S. Hassan, and S. K. Inouye, 'Sustainability and Scalability of the Hospital Elder Life Program at a Community Hospital', J Am Geriatr Soc, 59 (2011), 359-65.

T. R. Sanders, C. L. Roberts, and G. L. Gilbert, 'Compliance with a Protocol for Intrapartum Antibiotic Prophylaxis against Neonatal Group B Streptococcal Sepsis in Women with Clinical Risk Factors', Infectious Diseases in Obstetrics \& Gynecology, 10 (2002), 223-9.

A. P. Savant, L. J. Britton, K. Petren, S. A. McColley, and H. H. Gutierrez, 'Sustained Improvement in Nutritional Outcomes at Two Paediatric Cystic Fibrosis Centres after Quality Improvement Collaboratives', BMJ Quality and Safety, 23 (2014), i81-i89.

S. Scheithauer, F. Eitner, H. Hafner, J. Floege, and S. W. Lemmen, 'Long-Term Sustainability Timeframe $<1$ of Hand Hygiene Improvements in the Hemodialysis Setting', Infection, 41 (2013), 675-80. year Timeframe $<1$ year

clinical practice guideline

Timeframe $<1$ year

Timeframe unclear

Timeframe $<1$ year

No adherence studied

Short term and long term data were presented combined

Timeframe $<1$ year 
L. H. Schwamm, G. C. Fonarow, M. J. Reeves, W. Pan, M. R. Frankel, E. E. Smith, G. Ellrodt, No focus on a C. P. Cannon, L. Liang, E. Peterson, and K. A. LaBresh, 'Get with the Guidelines-Stroke Is Associated with Sustained Improvement in Care for Patients Hospitalized with Acute clinical practice Stroke or Transient Ischemic Attack', Circulation, 119 (2009), 107-15.

L. Bouadma, B. Mourvillier, V. Deiler, B. Le Corre, I. Lolom, B. Regnier, M. Wolff, and J. C. $\quad$ Timeframe $<1$ Lucet, 'A Multifaceted Program to Prevent Ventilator-Associated Pneumonia: Impact on year Compliance with Preventive Measures', Critical Care Medicine, 38 (2010), 789-96.

L. A. Shrier, S. A. Moszczenski, S. J. Emans, M. R. Laufer, and E. R. Woods, 'Three Years of a No adherence Clinical Practice Guideline for Uncomplicated Pelvic Inflammatory Disease in Adolescents', studied Journal of Adolescent Health, 27 (2000), 57-62.

K. C. Stange, M. A. Goodwin, S. J. Zyzanski, and A. J. Dietrich, 'Sustainability of a PracticeIndividualized Preventive Service Delivery Intervention', Am J Prev Med, 25 (2003), 296300 .

P. Sunaert, H. Bastiaens, F. Nobels, L. Feyen, G. Verbeke, E. Vermeire, J. De Maeseneer, S. Willems, and A. De Sutter, 'Effectiveness of the Introduction of a Chronic Care ModelBased Program for Type 2 Diabetes in Belgium', BMC Health Serv Res, 10 (2010), 207.

K. Swain, R. Whitley, G. J. McHugo, and R. E. Drake, 'The Sustainability of Evidence-Based Practices in Routine Mental Health Agencies', Community Ment Health J, 46 (2010), 11929.

M. A. Swales, B. Taylor, and R. A. B. Hibbs, 'Implementing Dialectical Behaviour Therapy: Programme Survival in Routine Healthcare Settings', Journal of Mental Health, 21 (2012), 548-55.

T. R. Talbot, J. G. Johnson, C. Fergus, J. H. Domenico, W. Schaffner, T. L. Daniels, G. Wilson, J. Slayton, N. Feistritzer, and G. B. Hickson, 'Sustained Improvement in Hand Hygiene Timeframe $<1$ year

Timeframe unclear

Short term and long term data were presented combined

No adherence studied Adherence: Utilizing Shared Accountability and Financial Incentives', Infection Control and Hospital Epidemiology, 34 (2013), 1129-36.

D. R. Taylor, 'Sustained Success of Collaboratively Developed Ondansetron Guidelines', Australian Journal of Hospital Pharmacy, 25 (1995), 522-25.

Insufficient information provided

J. Tjia, C. Lemay, K. M. Mazor, B. A. Briesacher, T. S. Field, A. O. Kanaan, J. L. Donovan, L. R. Timeframe $<1$ Harrold, and J. H. Gurwitz, 'Dissemination of Evidence-Based Antipsychotic Prescribing year Guidelines to Nursing Homes: A Cluster Randomized Trial', Journal of the American Geriatrics Society, 62 (2014), S128.

A. Mukerji, J. Narciso, C. Moore, A. McGeer, E. Kelly, and V. Shah, 'An Observational Study Timeframe $<1$ of the Hand Hygiene Initiative: A Comparison of Preintervention and Postintervention year Outcomes', BMJ Open, 3 (2013).

R. Van Acker, I. De Bourdeaudhuij, K. De Cocker, L. M. Klesges, A. Willem, and G. Cardon, No adherence 'Sustainability of the Whole-Community Project '10,000 Steps': A Longitudinal Study', BMC studied Public Health, 12 (2012), 155. 
Paper Exclusion reason

D. Pittet, S. Hugonnet, S. Harbarth, P. Mourouga, V. Sauvan, S. Touveneau, and T. V. $\quad$ Timeframe $<1$ Perneger, 'Effectiveness of a Hospital-Wide Programme to Improve Compliance with Hand year Hygiene. Infection Control Programme.[Erratum Appears in Lancet 2000 Dec 2330;356(9248):2196]', Lancet, 356 (2000), 1307-12.

B. W. Warner, K. A. Rich, H. Atherton, C. L. Andersen, and U. R. Kotagal, 'The Sustained No adherence Impact of an Evidenced-Based Clinical Pathway for Acute Appendicitis', Seminars in studied Pediatric Surgery, 11 (2002), 29-35.

F. H. van Tiel, T. W. O. Elenbaas, B. M. A. M. Voskuilen, J. Herczeg, F. W. Verheggen, B. Mochtar, and E. E. Stobberingh, 'Plan-Do-Study-Act Cycles as an Instrument for Timeframe $<1$ year Improvement of Compliance with Infection Control Measures in Care of Patients after Cardiothoracic Surgery', Journal of Hospital Infection, 62 (2006), 64-70.

B. W. Trautner, N. J. Petersen, A. Gendrett, S. Hysong, J. E. Patterson, and A. D. Naik, 'An Audit/Feedback Intervention Reduces Inappropriate Screening and Treatment in Patients with Urinary Catheters', Journal of the American Geriatrics Society, 62 (2014), S125.

A. L. Wentworth, C. H. Fox, L. S. Kahn, K. Glaser, and R. Cadzow, 'Two Years after a Quality No adherence Improvement Intervention for Chronic Kidney Disease Care in a Primary Care Office', $\quad$ studied American Journal of Medical Quality, 26 (2011), 200-05.

M. Whitby, M. L. McLaws, K. Slater, E. Tong, and B. Johnson, 'Three Successful Interventions in Health Care Workers That Improve Compliance with Hand Hygiene: Is Sustained Replication Possible?', American Journal of Infection Control, 36 (2008), 349-55.

S. Whitelaw, N. Graham, D. Black, J. Coburn, and L. Renwick, 'Developing Capacity and Achieving Sustainable Implementation in Healthy 'Settings': Insights from Nhs Health Scotland's Health Promoting Health Service Project', Health promotion international, 27 (2012), 127-37.

L. J. Weireter, Jr., J. N. Collins, R. C. Britt, S. F. Reed, T. J. Novosel, and L. D. Britt, 'Impact of Timeframe a Monitored Program of Care on Incidence of Ventilator-Associated Pneumonia: Results of unclear a Longterm Performance-Improvement Project', Journal of the American College of Surgeons, 208 (2009), 700-4; discussion 04-5.

R. B. Womer, E. Tracy, W. Soo-Hoo, B. Bickert, S. DiTaranto, and J. H. Barnsteiner, No adherence 'Multidisciplinary Systems Approach to Chemotherapy Safety: Rebuilding Processes and studied Holding the Gains', Journal of Clinical Oncology, 20 (2002), 4705-12.

D. M. Zerr, A. L. Allpress, J. Heath, R. Bornemann, and E. Bennett, 'Decreasing HospitalAssociated Rotavirus Infection: A Multidisciplinary Hand Hygiene Campaign in a Children's year Hospital', Pediatric Infectious Disease Journal, 24 (2005), 397-403.

N. Zwar, J. Henderson, H. Britt, K. McGeechan, and G. Yeo, 'Influencing Antibiotic No focus on a Prescribing by Prescriber Feedback and Management Guidelines: A 5-Year Follow-Up', in clinical practice Family practice (2002), pp. 12-7. guideline 
SUSTAINABILITY OF PROFESSIONALS' ADHERENCE TO GUIDELINES

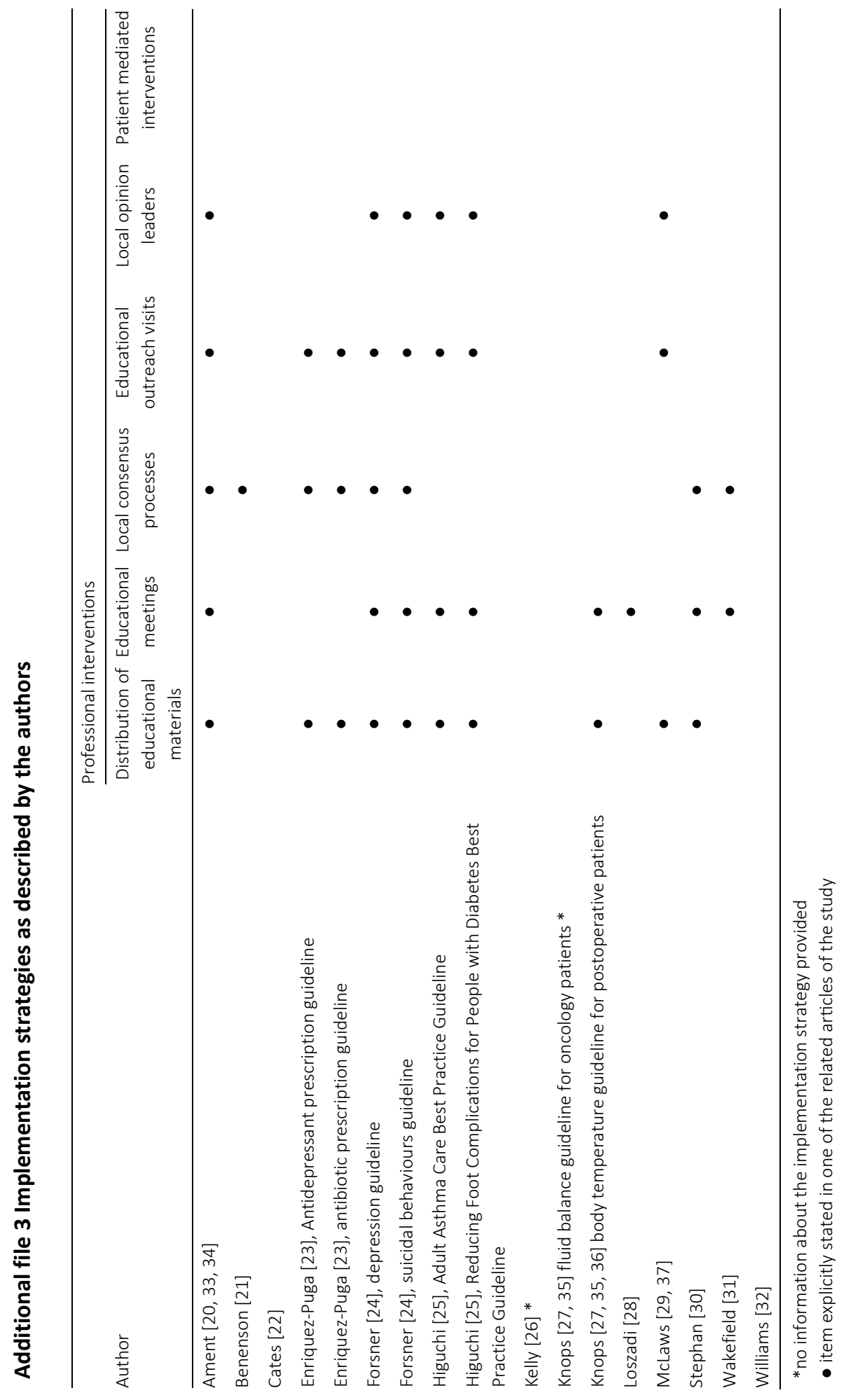


CHAPTER 6

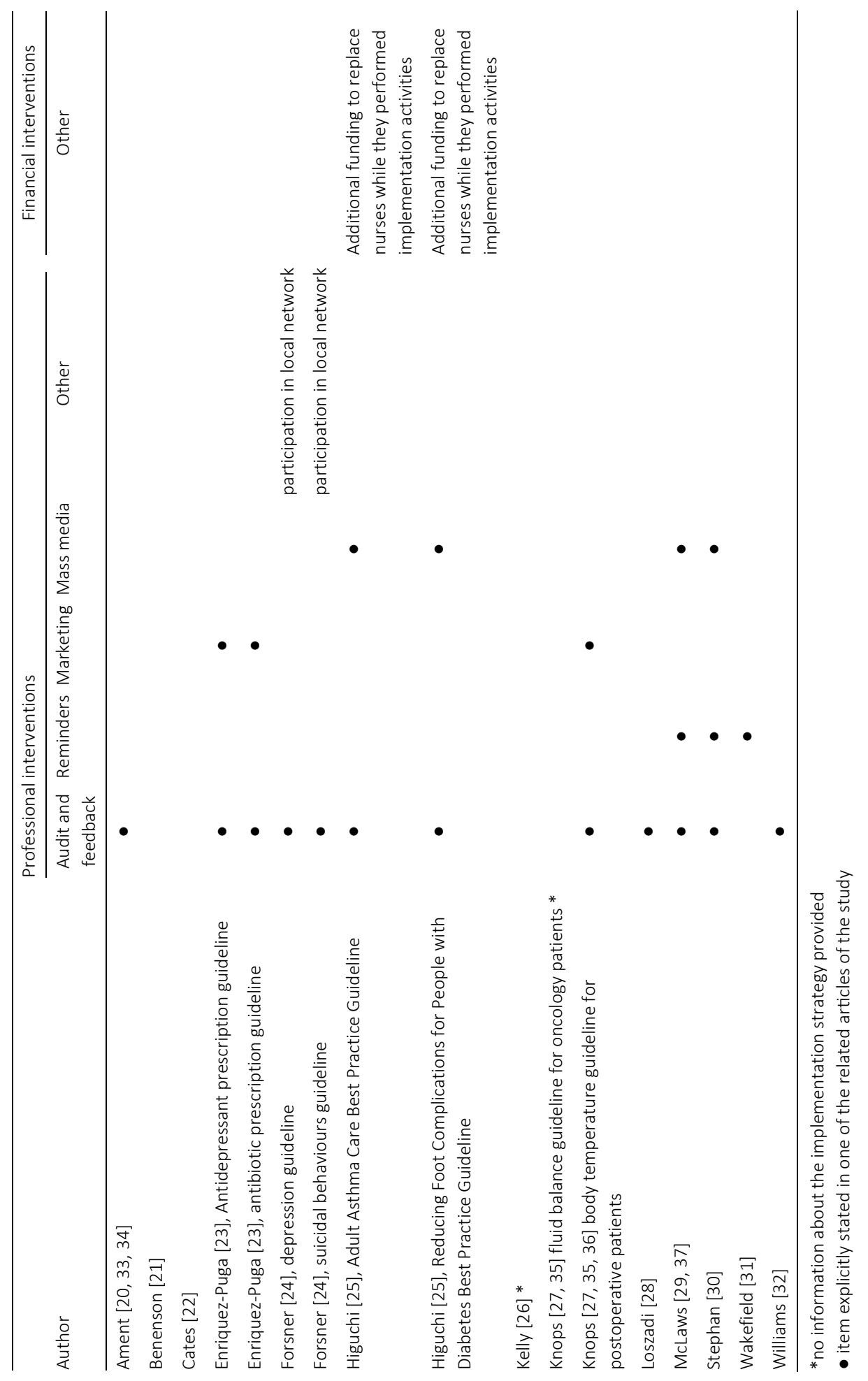


SUSTAINABILITY OF PROFESSIONALS' ADHERENCE TO GUIDELINES

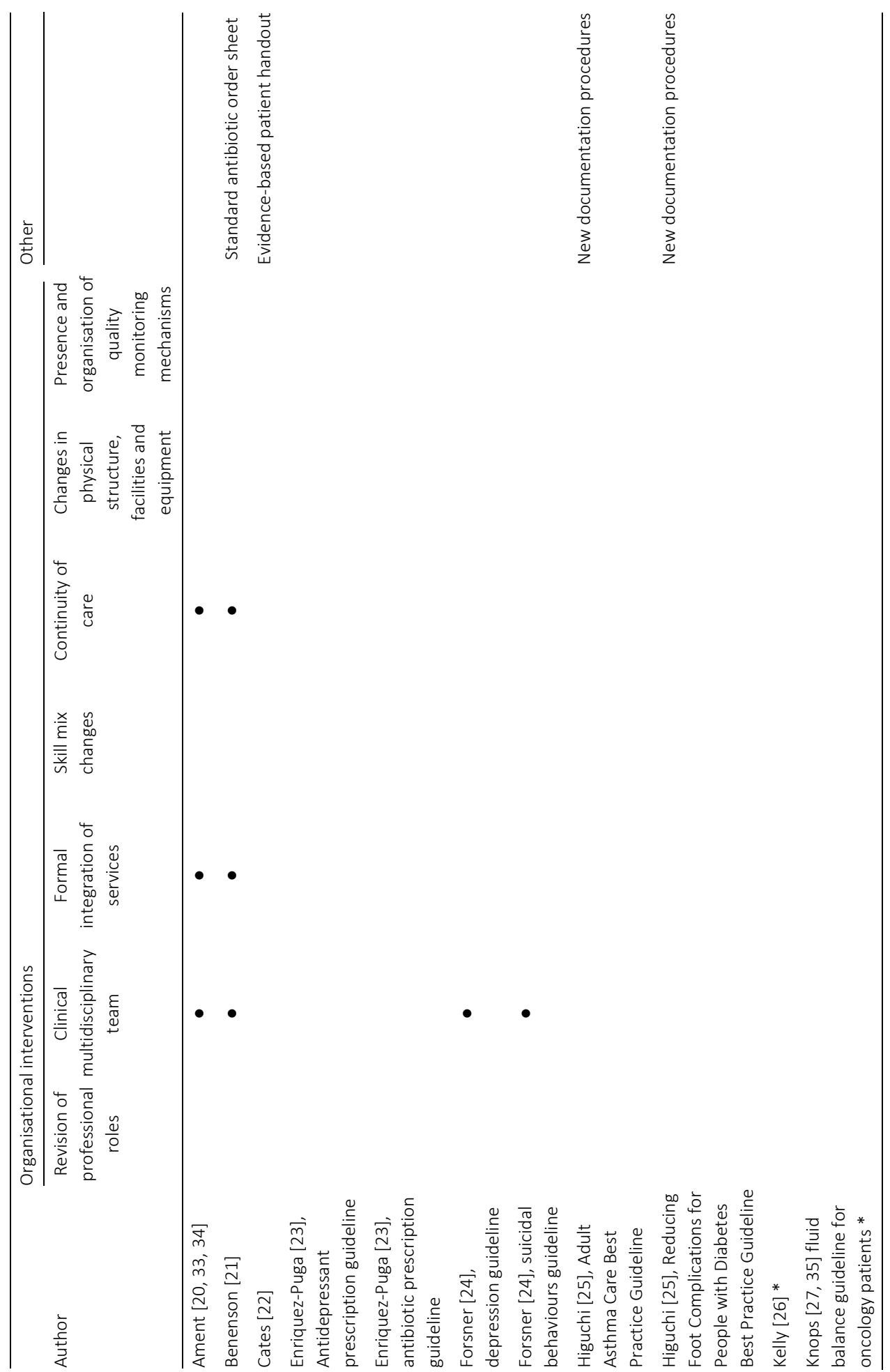




\section{CHAPTER 6}
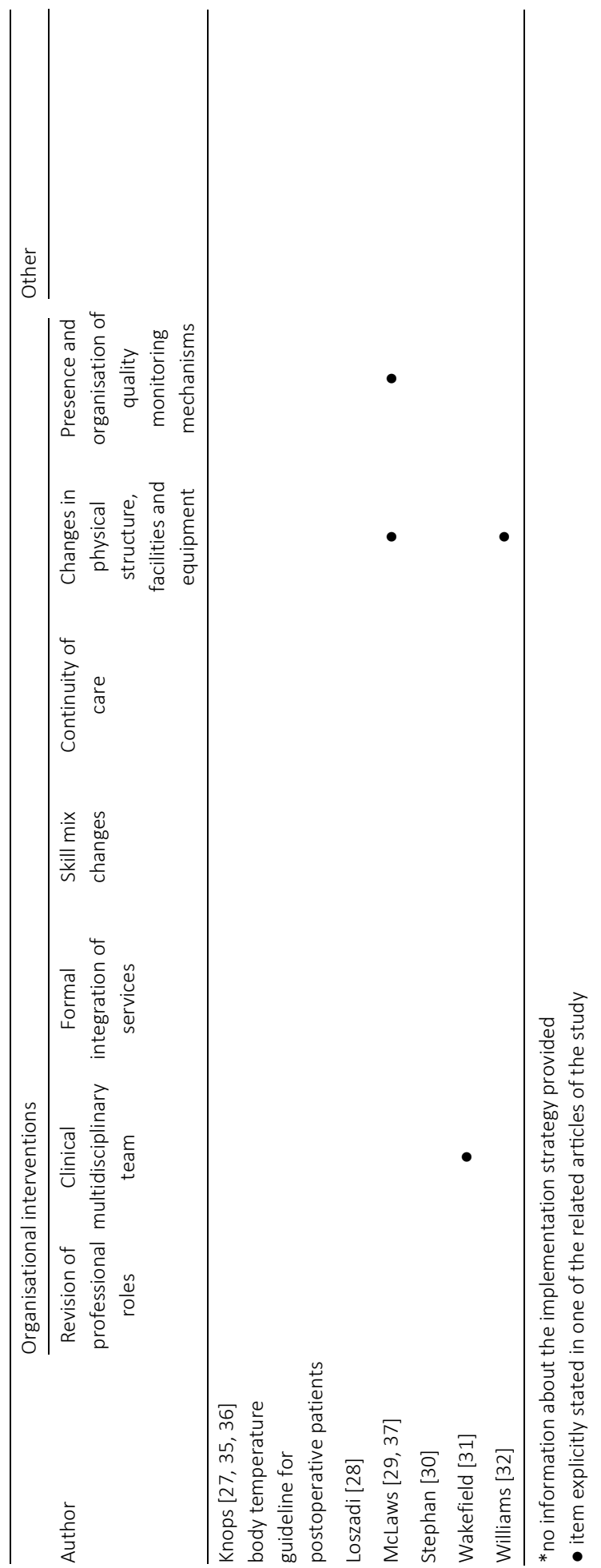
SUSTAINABILITY OF PROFESSIONALS' ADHERENCE TO GUIDELINES

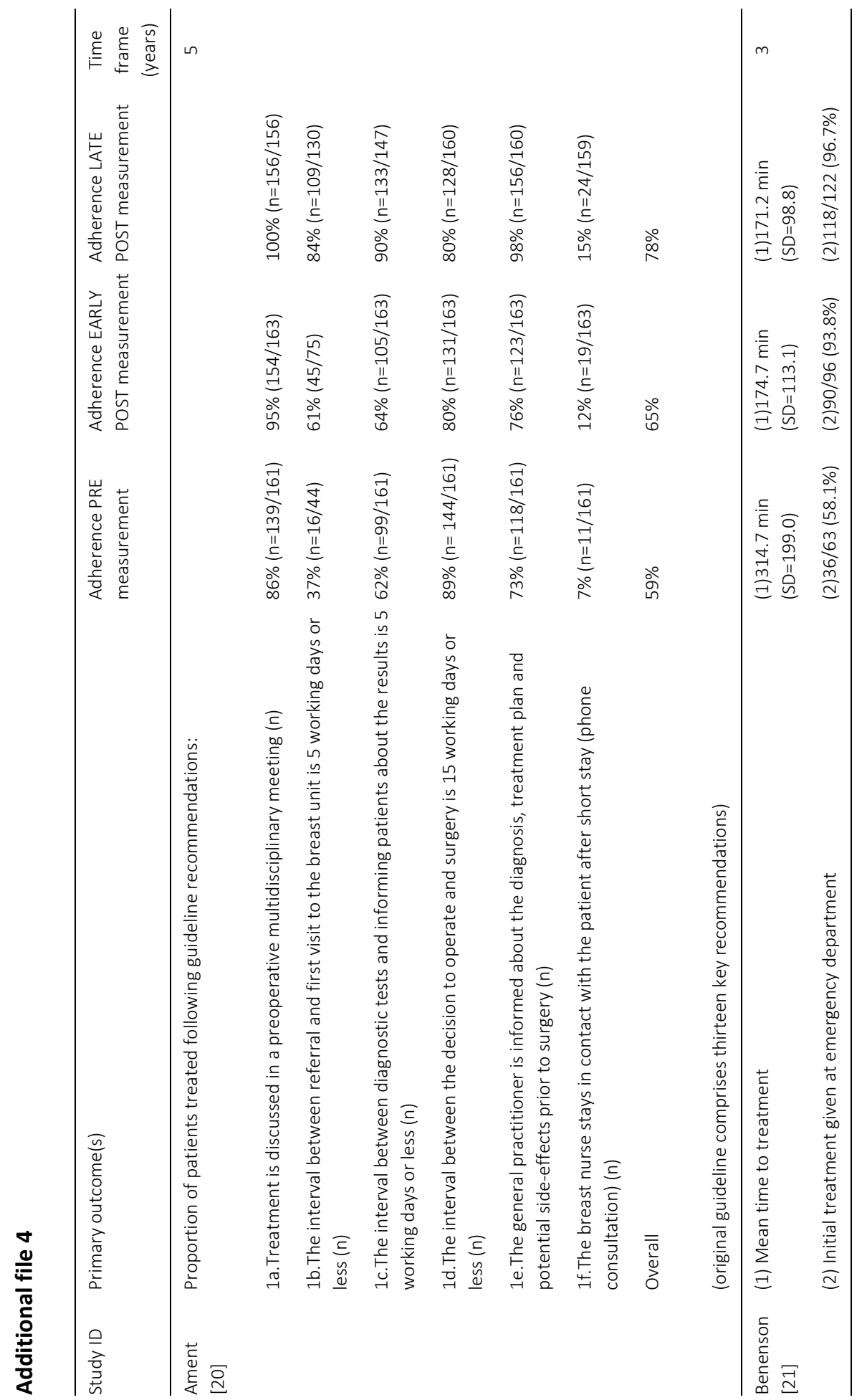


CHAPTER 6

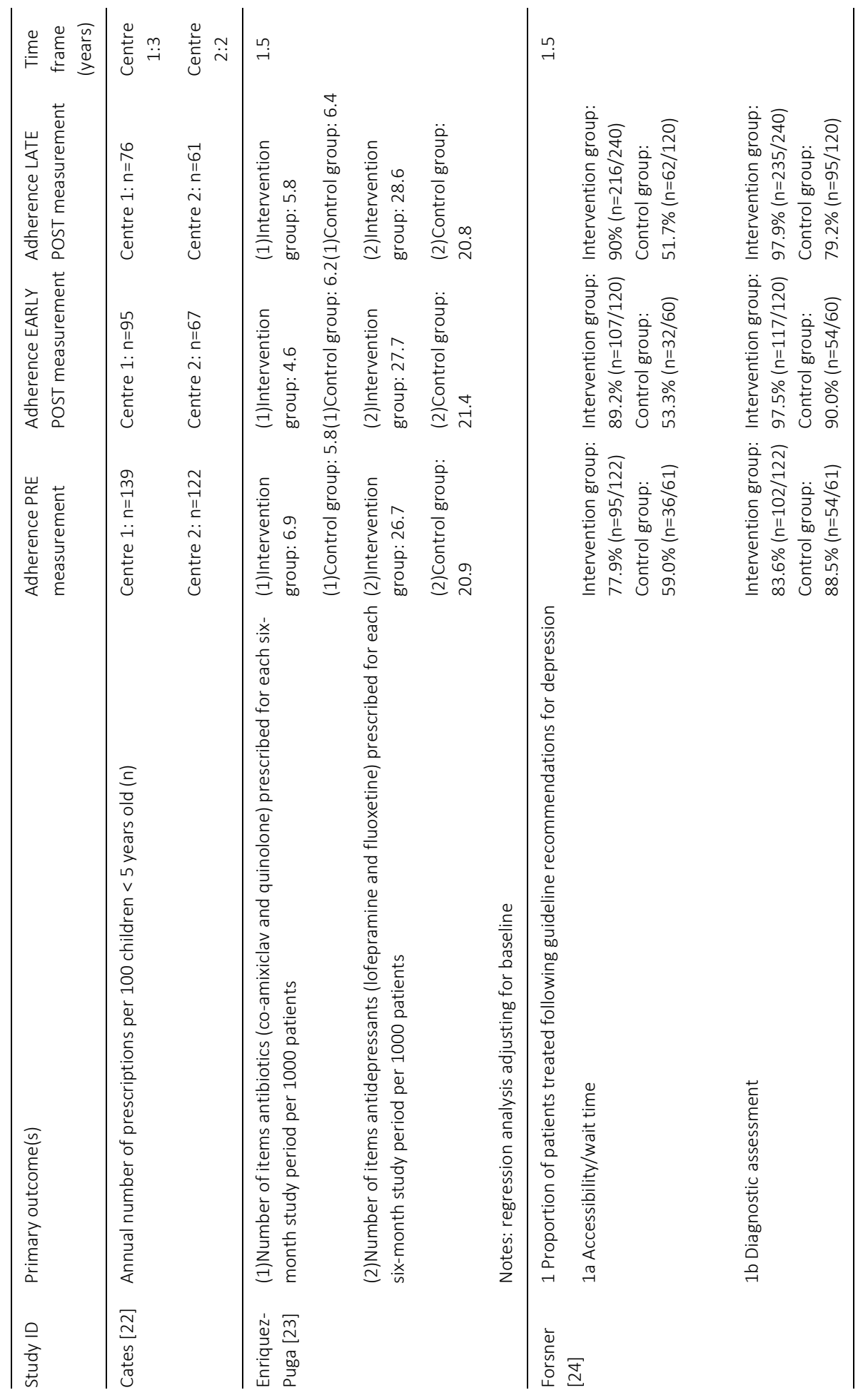


SUSTAINABILITY OF PROFESSIONALS' ADHERENCE TO GUIDELINES

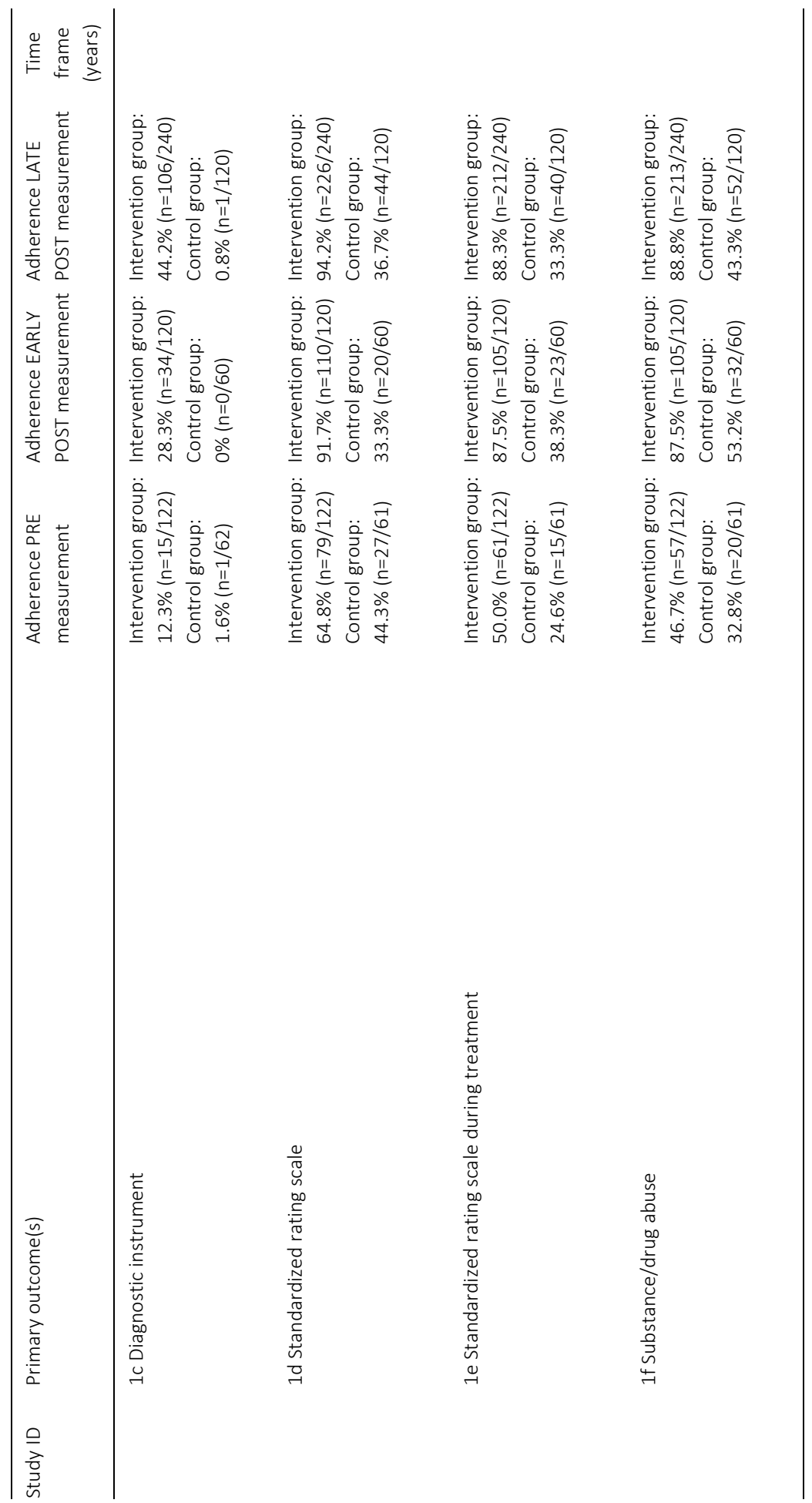


CHAPTER 6

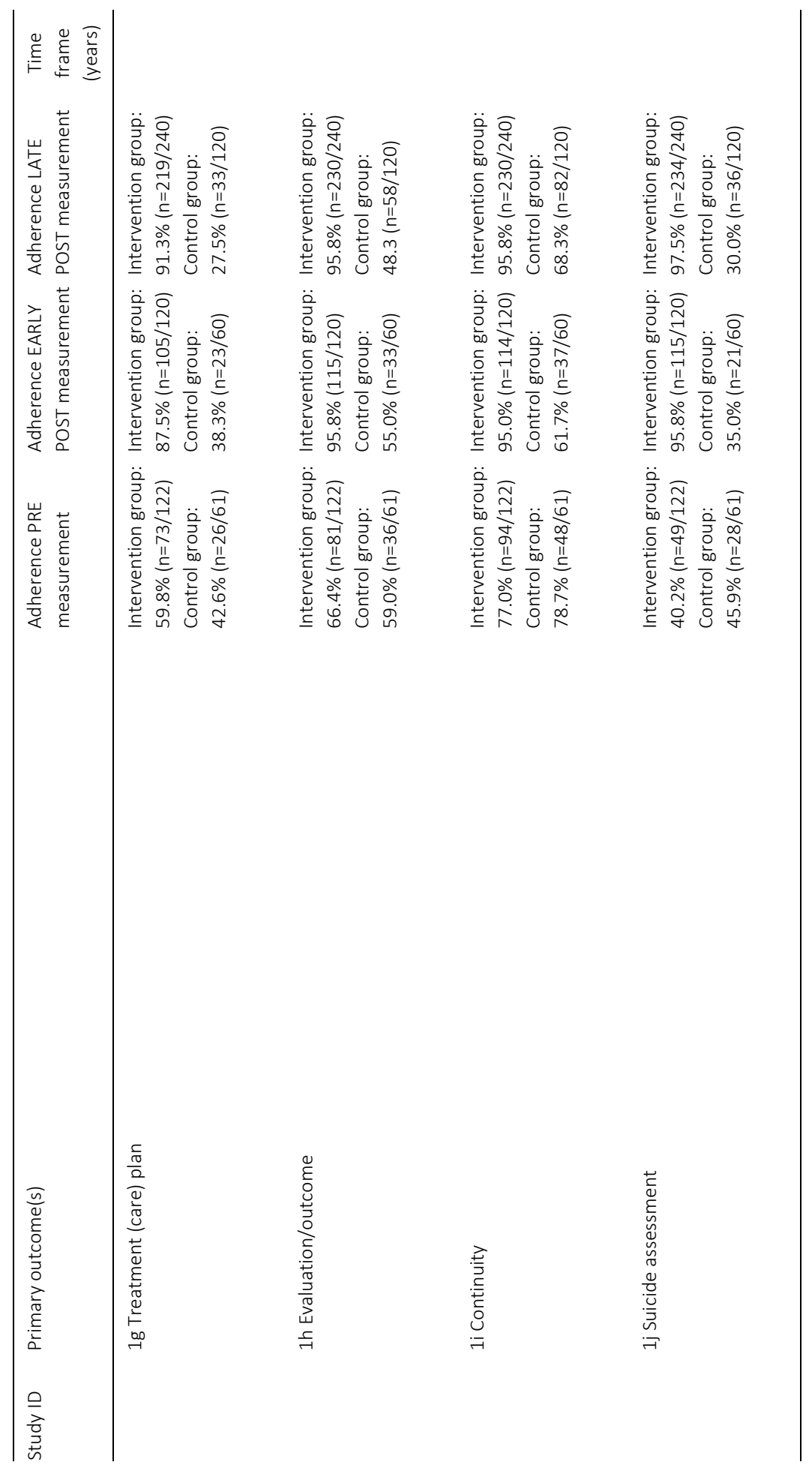


SUSTAINABILITY OF PROFESSIONALS' ADHERENCE TO GUIDELINES

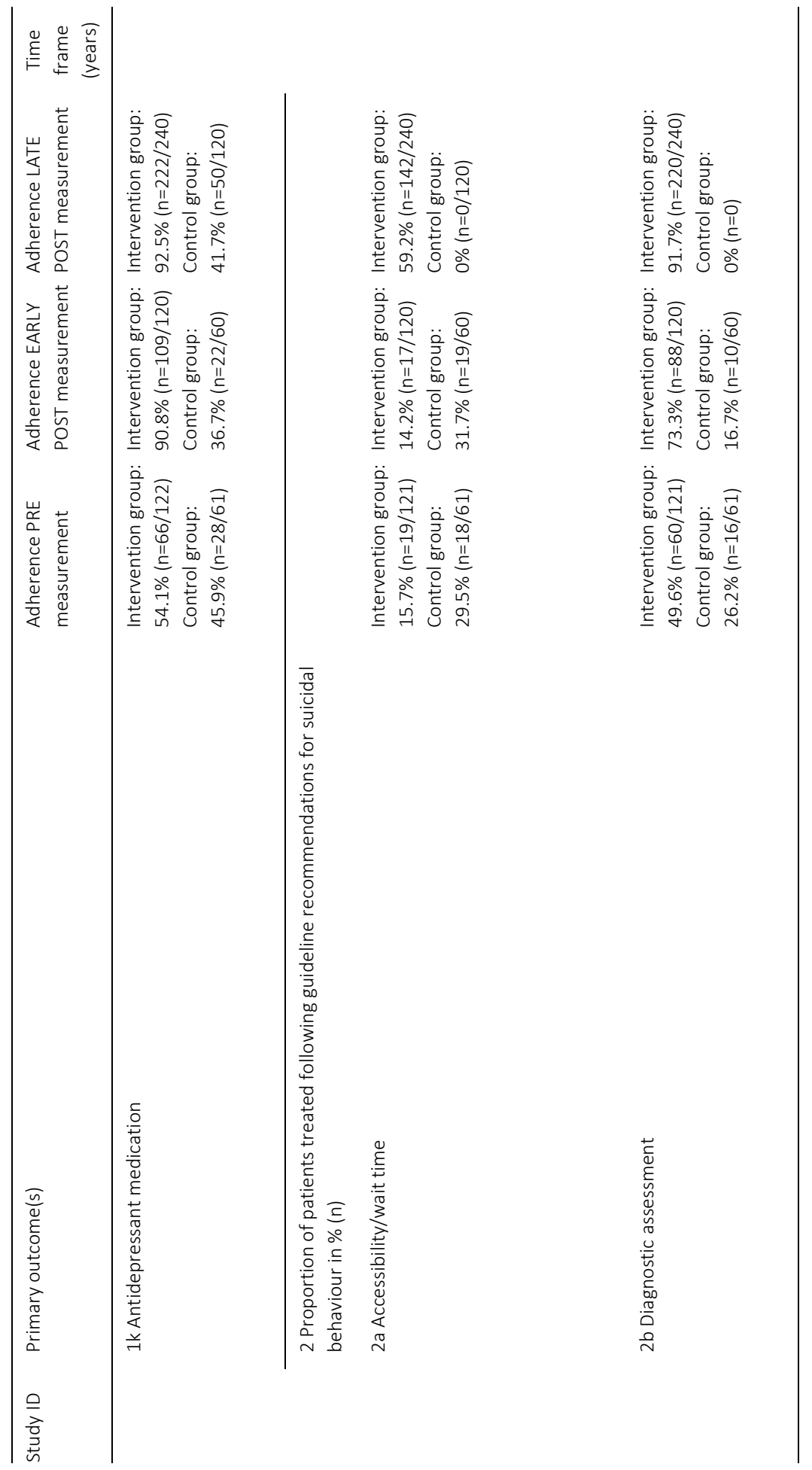


CHAPTER 6

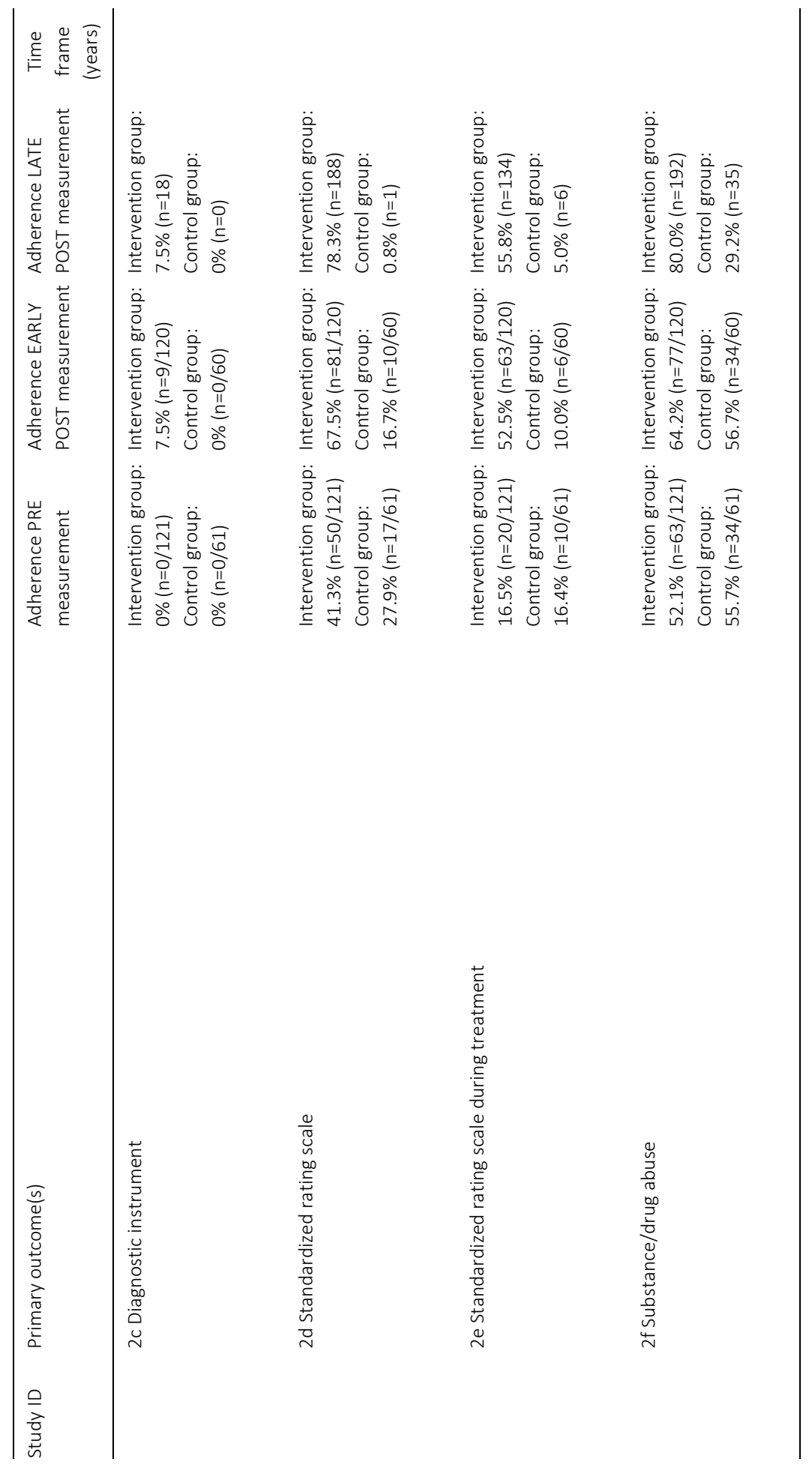


SUSTAINABILITY OF PROFESSIONALS' ADHERENCE TO GUIDELINES

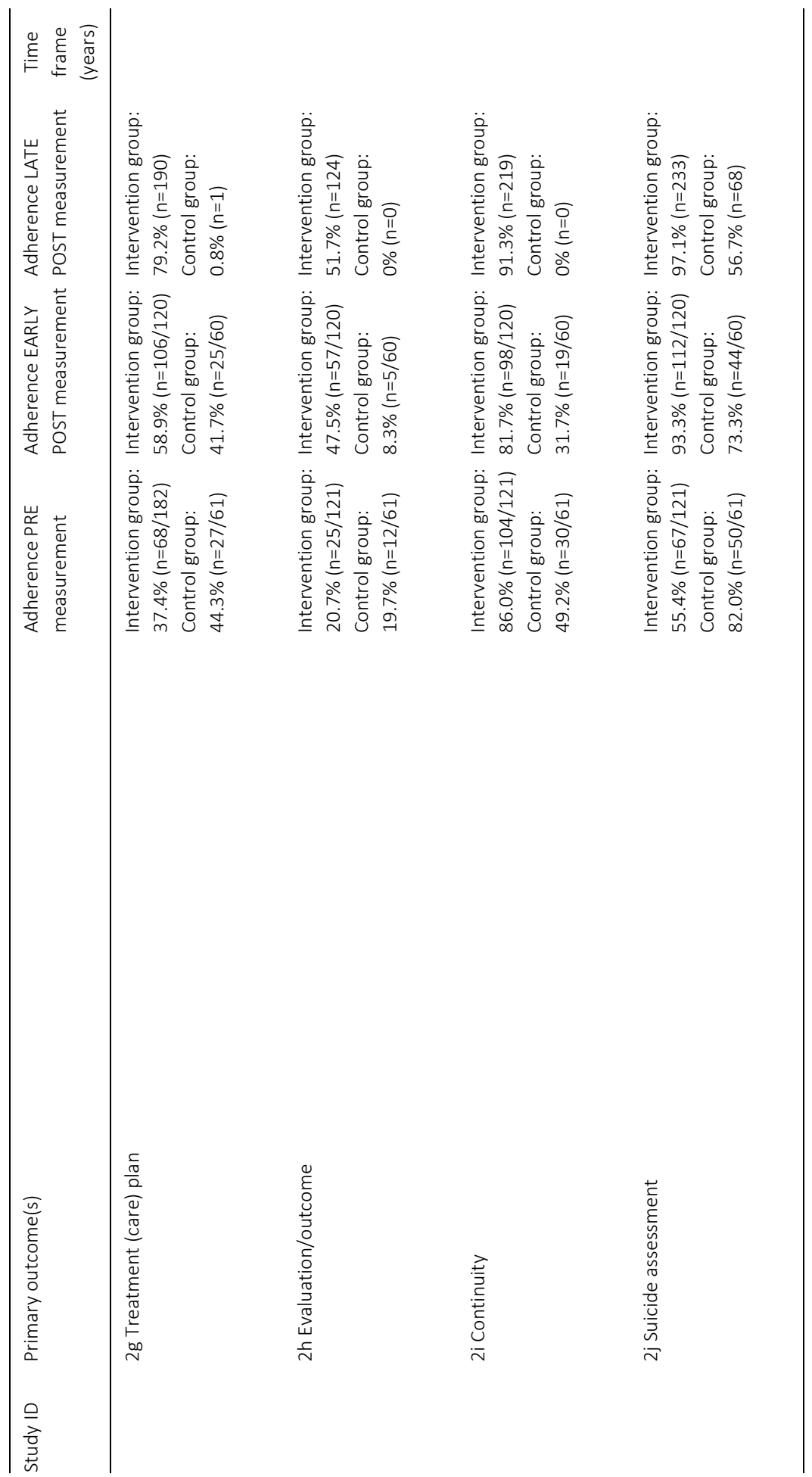


CHAPTER 6

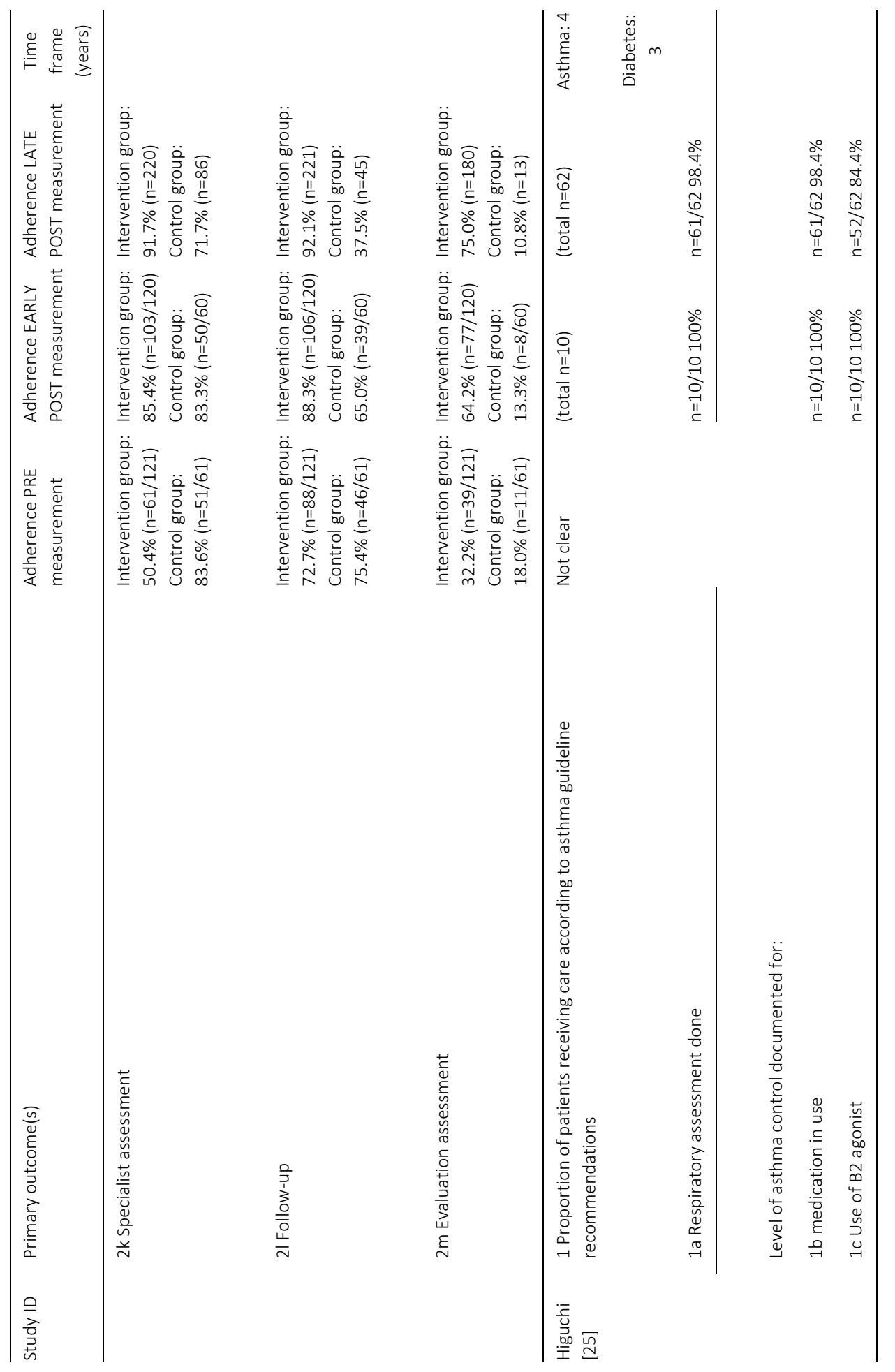


SUSTAINABILITY OF PROFESSIONALS' ADHERENCE TO GUIDELINES

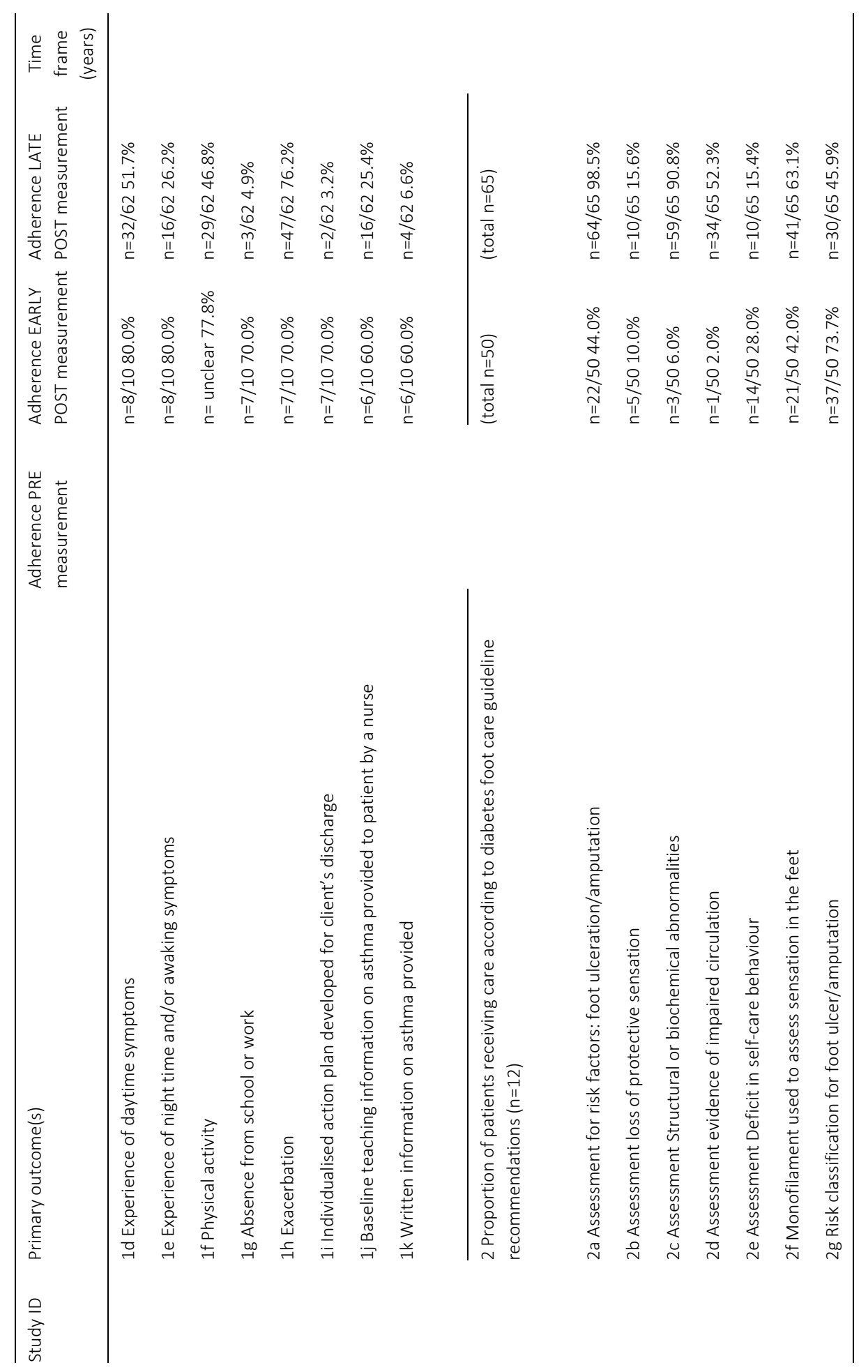


CHAPTER 6

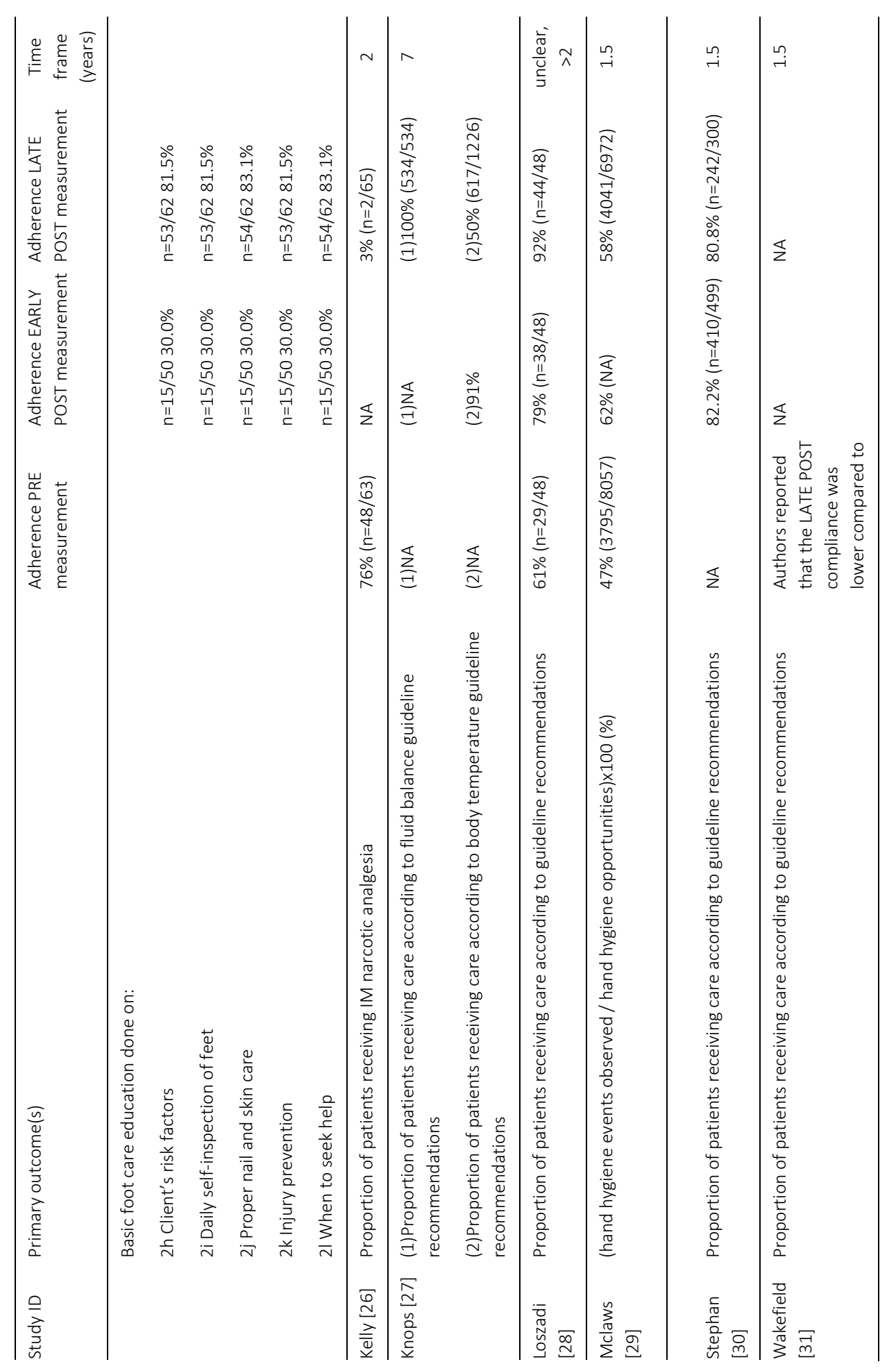


SUSTAINABILITY OF PROFESSIONALS' ADHERENCE TO GUIDELINES

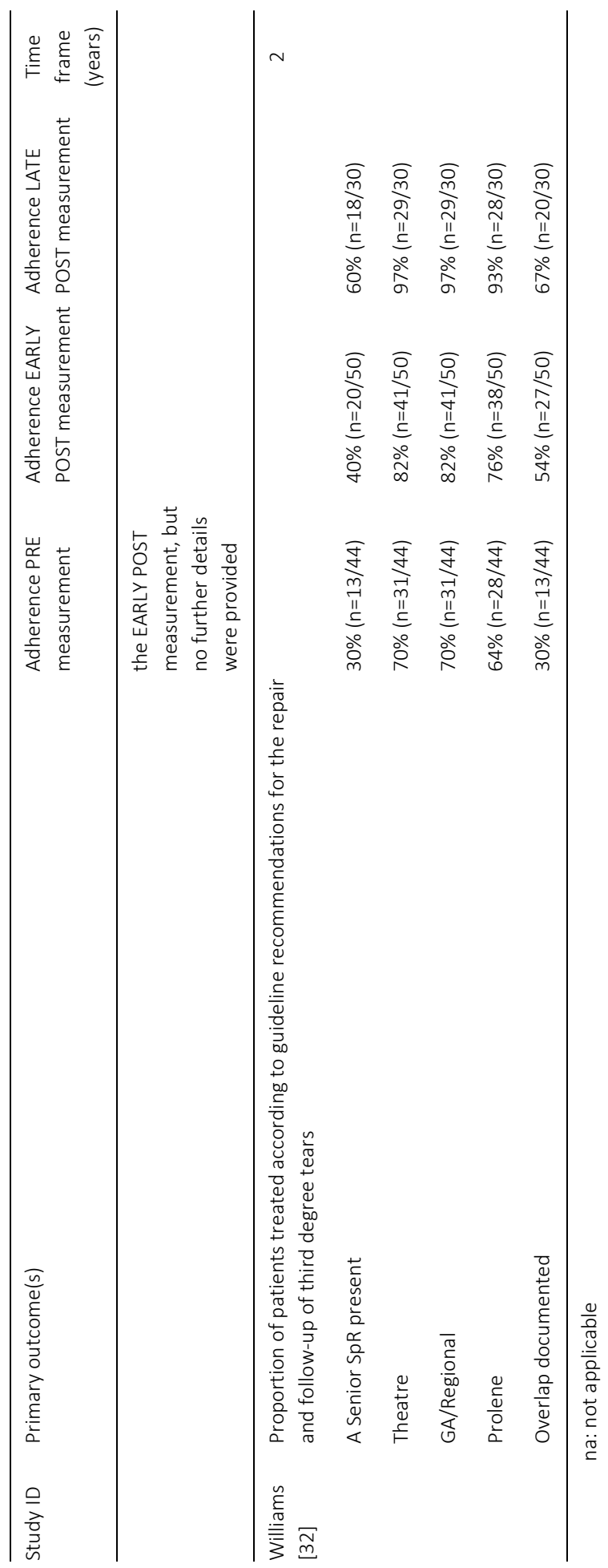




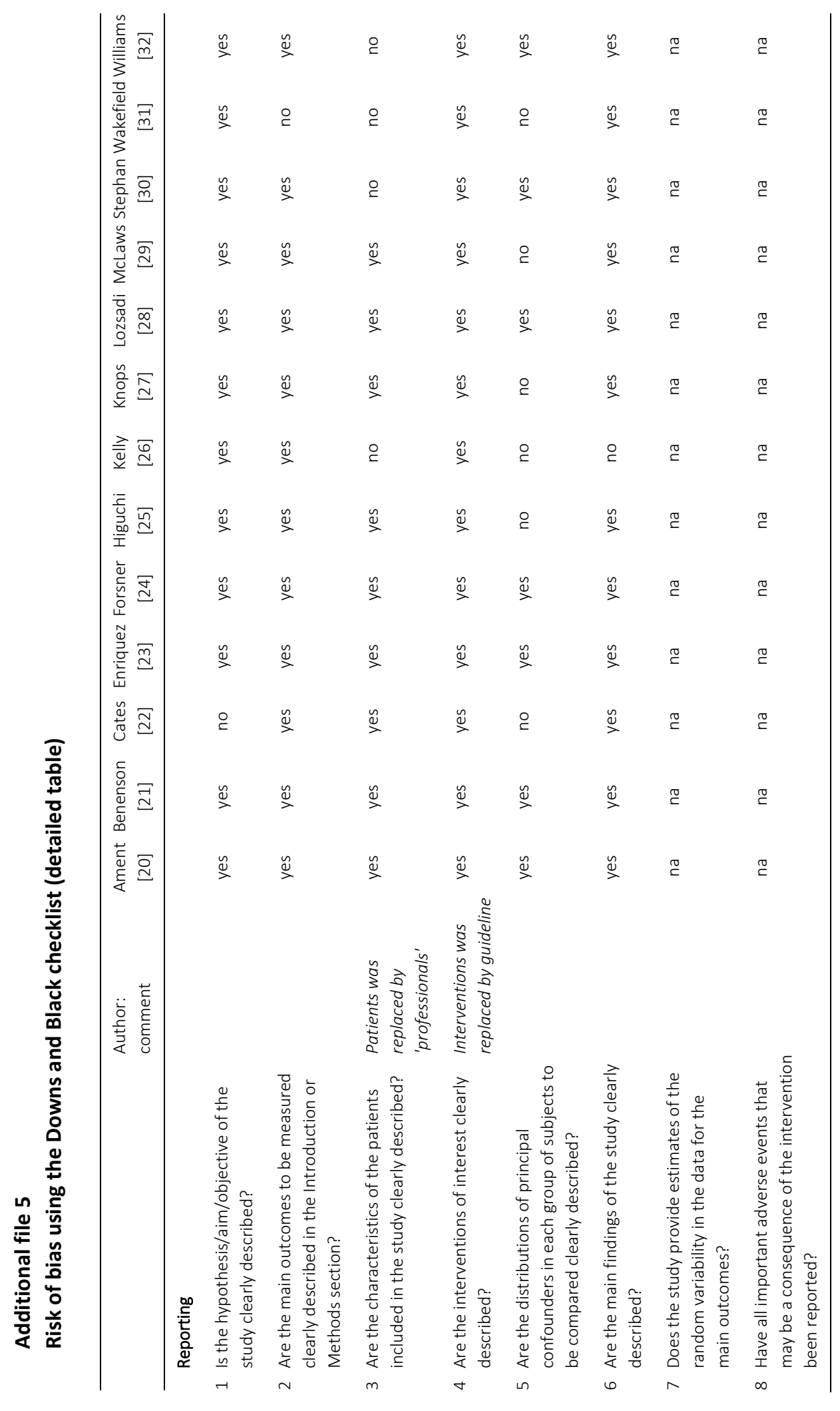




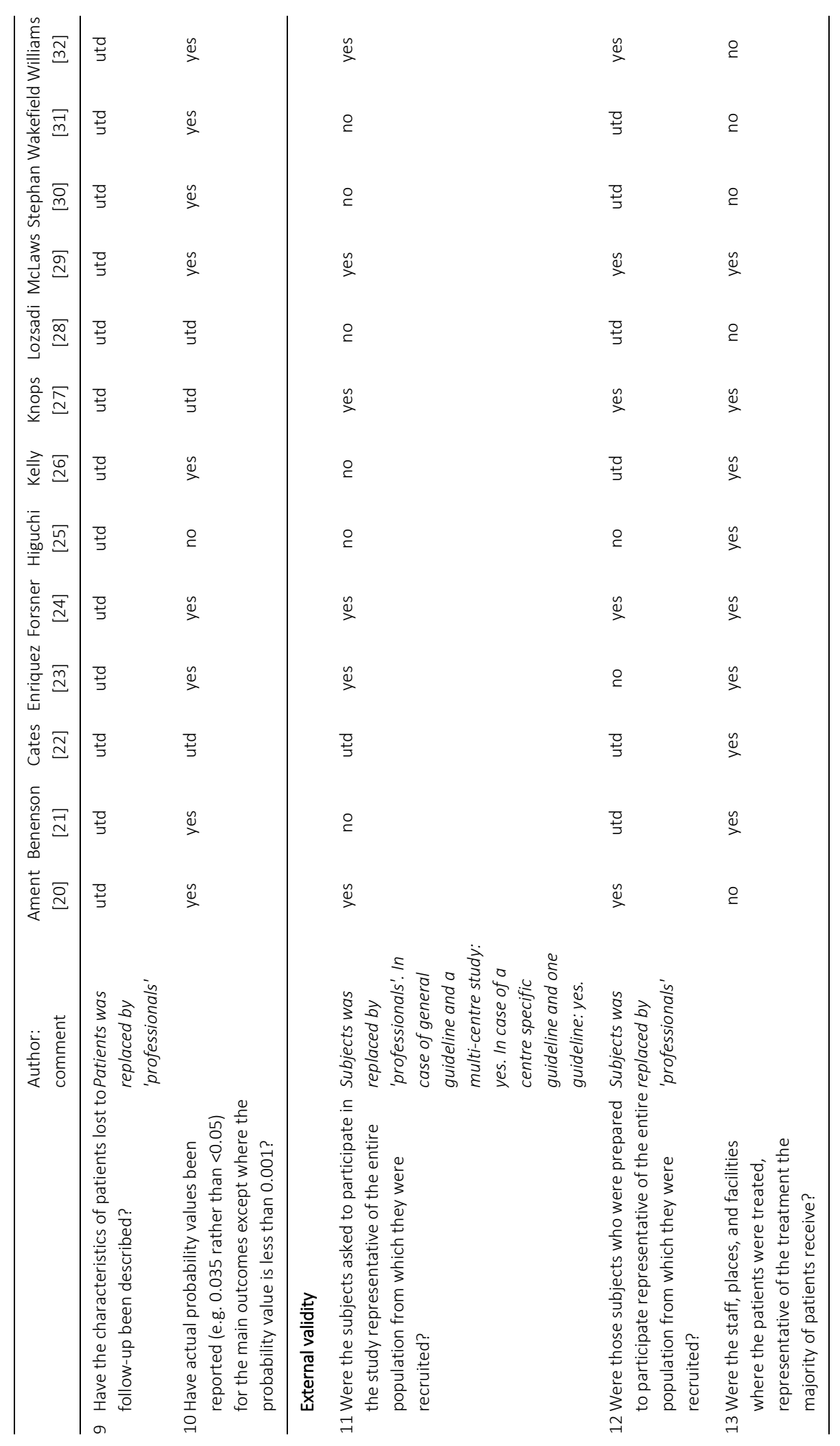




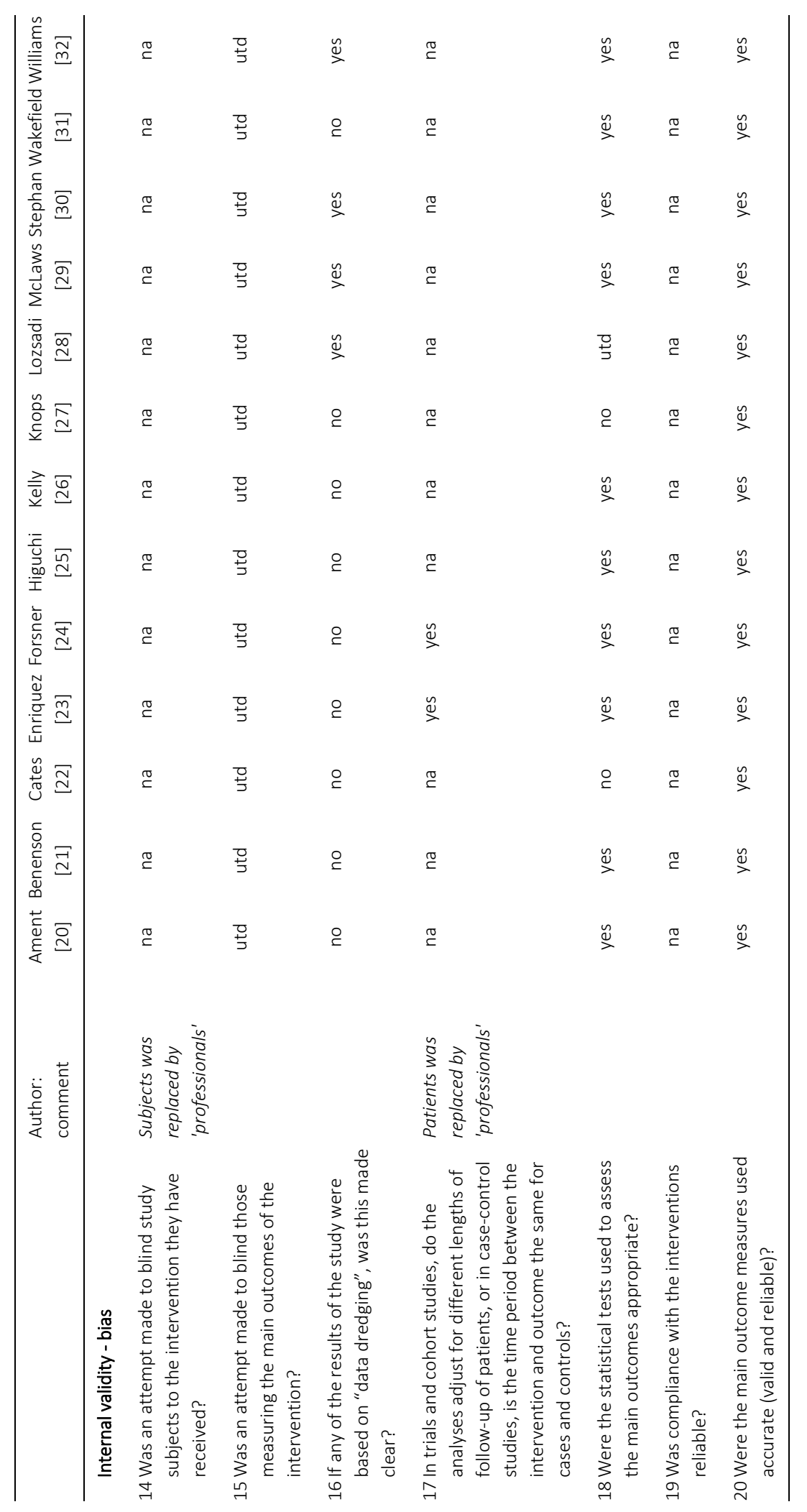




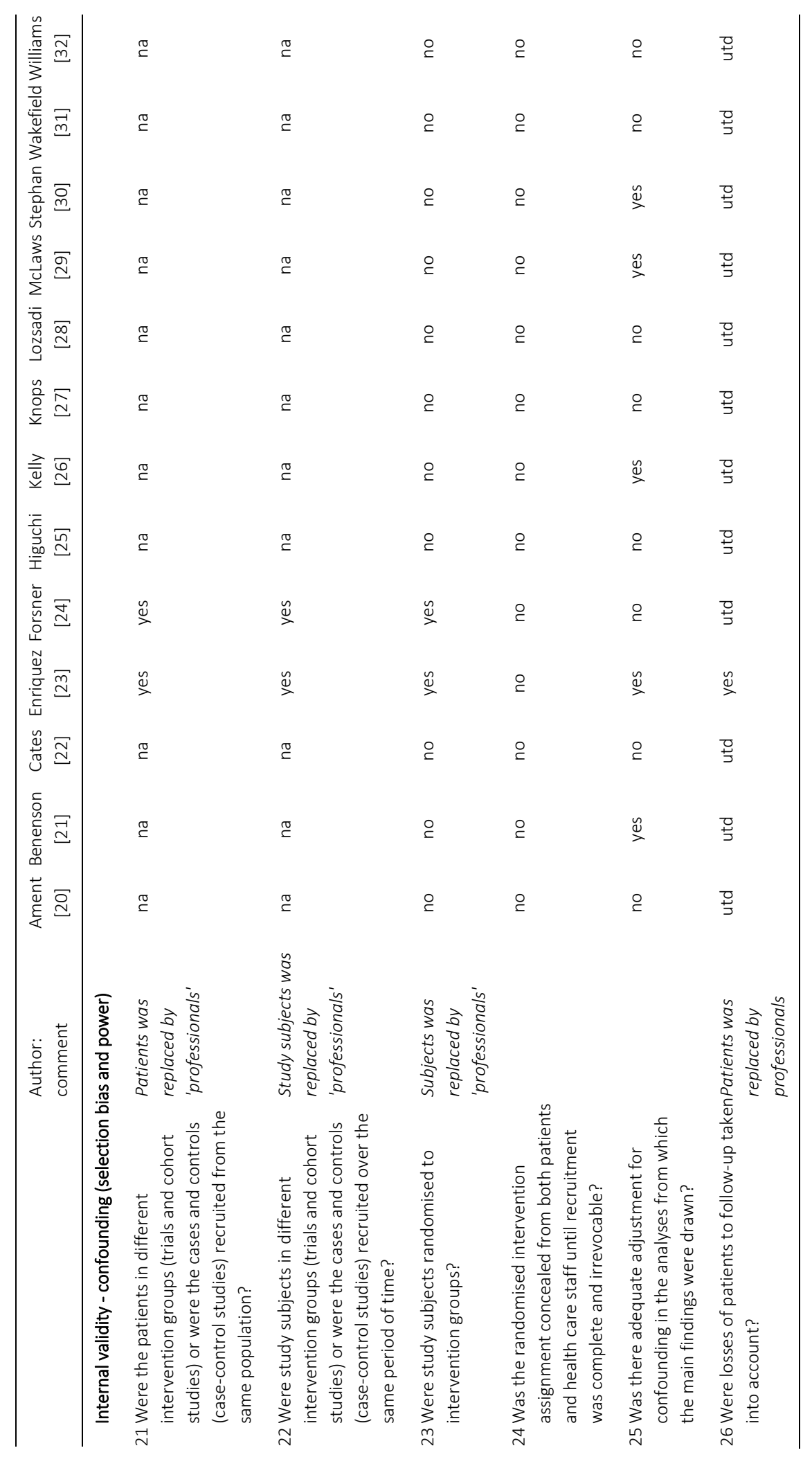




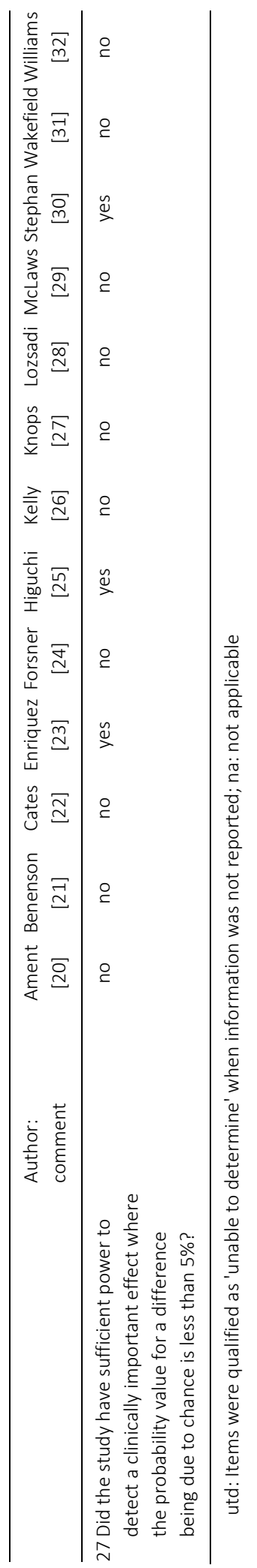




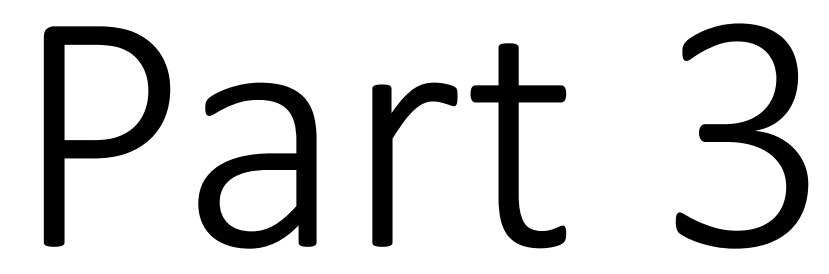

Qualitative analyses of sustainability 



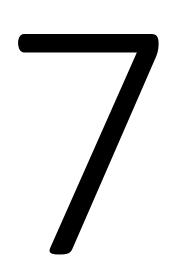

\section{What determines sustainability of two multidisciplinary hospital-based programmes after achieving early implementation success? A qualitative study}

* both authors contributed equally 


\section{CHAPTER 7}

\section{Abstract}

\section{Background}

Sustainability of innovations is a relatively new concept in health care research and has become an issue of growing interest. The current study explored determinants of the sustainability of two multidisciplinary hospital-based programmes three to six years after achieving early implementation success.

\section{Methods}

An exploratory qualitative study was conducted into two implementation cases, an Enhanced Recovery After Surgery (ERAS) programme for colonic surgery and a short stay programme for breast cancer surgery (SSP). Semi-structured interviews were held with key persons involved in the care process minimally three years after the implementation, between March 2012 and May 2013. The Consolidated Framework for Implementation Research (CFIR) was used to direct the development of the interview guide, during data collection and during analysis. A direct content analysis was performed.

\section{Results}

A total of 21 interviews with 26 individuals were held, 18 regarding the ERAS case and eight regarding the SSP case. Respondents mentioned the following key determinants of sustainability of the programmes: modification and adaptability of the programme, cost-effectiveness, institutionalisation into existing systems, short communication lines within the multidisciplinary team, an innovative culture, benefits for patients, cosmopolitanism, the existence of external policies and incentives, trust and belief in the programme, and spread of the programme to other settings. Two of these determinants are not covered by the CFIR, i.e. modification of the programme over the years and spread of the programme to other contexts.

\section{Conclusions}

The key determinants of sustainability put forward were largely the same in both cases. Leadership and the implementation project were not mentioned as having influenced the long-term sustainability of the benefits achieved. Sustainability of the innovations is influenced by determinants stemming from all ecological levels of the health care system and demands continuous effort in the post-implementation phase. 


\section{Background}

Quality of care is an important subject in health care research and quality improvement strategies are frequently used to change and improve health care. Once the process of implementation is completed, however, there is often a tendency to relapse into old routines [1]. Quality improvement efforts are a waste of time and money if early postimplementation successes, that is, the benefits achieved immediately after the end of an implementation project, are lost again in the post-implementation phase. Moreover, the consequences of evaporation of early post-implementation successes are not just financial but may also result in suboptimal care, cause frustration and diminish the support for future health care innovations. It is therefore important that implementation successes are sustained over a longer period of time.

Sustainability of innovations is a relatively new term in health care research and has become an issue of growing interest [2,3]. In its simplest form, it can be seen as 'normalisation', 'holding the gains' and 'evolving as required'. Different definitions of sustainability have been proposed [4-8]. In the current study we use the following definition: 'Sustainability of change exists when a newly implemented innovation continues to deliver the achieved benefits over a longer period of time, certainly does not return to the usual processes and becomes "the way things are done around here", until a better innovation comes along, even after the implementation project is no longer actively carried out' [9]. Key determinants of the sustainability of early post-implementation successes are not necessarily the same as those of the achievement of the early successes. Maintaining early post-implementation successes, in terms of achieved benefits, in the real world involves a dynamic process $[6,8]$. A conceptualisation of three main determinants, viz. the intervention that is implemented, the organisation and the community and environment, was proposed by Scheirer [7] and has been used in most sustainability studies. Since innovations are implemented in various fields of health care, key determinants of the sustainability of health care programmes may vary considerably, and research into them is underdeveloped [10,11]. While most sustainability studies focused on results in public health during two or more years after the initial implementation [11], few have assessed the long-term sustainability of innovations in a multidisciplinary hospital-based setting [12].

We tried to identify the key determinants of the sustainability of two multidisciplinary hospital-based surgical care programmes, the enhanced recovery after surgery programme (ERAS) for colonic surgery and the short-stay programme (SSP) for breast cancer surgery $[13,14]$, by exploring the post-implementation results of these two programmes three to six years after successful implementation, and the views of key players on this process. The more general aim of this study was to explore determinants of sustainability of quality improvements in health care. Knowing and understanding these determinants should lead to more effective implementation and should help to understand, predict and increase the chances of long-term sustainability. 


\section{CHAPTER 7}

\section{Methods}

\section{Study design}

An exploratory qualitative two-case study was conducted to identify determinants of the sustainability of the programmes as perceived by professionals. The study protocol has been published elsewhere [9].

\section{Framework}

In the current study, the Consolidated Framework for Implementation Research (CFIR), developed by Damschroder et al $[15,16]$, was used as a systematic approach gathering and analysing data regarding the determinants of sustainability of two surgical programmes as perceived by professionals. The CFIR provides a broad comprehensive model of evidence-based constructs which cover the full context of implementation, and also considers a broad scope of potential determinants of sustainability. The CFIR is composed of 39 constructs, organised into five main domains: the characteristics of the innovation, the individuals involved, the inner setting, the outer setting and the implementation process [16]. The framework was used to direct the content of the interview guide and to guide the coding process of the interviews.

\section{Setting}

\section{Two implementation cases}

Two implementation cases were used for the exploration of the concept of sustainability as part of the main study [9]. The two implementation cases were selected as these were two prominent studies which were executed by the Maastricht University Medical Centre and had both been funded by The Netherlands Organisation for Health Research and Development.

\section{Enhanced Recovery After Surgery (ERAS)}

The ERAS programme describes eighteen elements of perioperative care and was developed to enhance functional recovery after surgery and to reduce the hospital length of stay (LOS) after colonic surgery $[17,18]$. The programme was implemented for colonic surgery in 33 hospitals in the Netherlands between 2006 and 2009. It was implemented by means of a generic implementation strategy, the Breakthrough Series. The Breakthrough Series, developed by the Institute for Health care Improvements (IHI), USA, reported to be an effective approach to implementing change in clinical medicine $[19,20]$. It involves a structured collaboration between hospitals that share the desire to attain a known best practice in a particular field. As a result of the implementation project, functional recovery after colonic surgery was enhanced, and hospital length of stay decreased from nine to six days. A recent quantitative evaluation of the long-term results showed that three to six years following its implementation, the sustainability of 
the early implementation results in terms of functional recovery, length of hospital stay and protocol adherence varied between hospitals [21].

\section{Short-stay programme for breast cancer surgery (SSP)}

SSP is a comprehensive programme and was developed to increase the efficiency of breast cancer surgery care by renewing the patient information strategy and by standardisation of the care process, while maintaining the perceived quality of care by patients [22]. The programme was implemented in four early adopter hospitals in the Netherlands between 2005 and 2007, by means of a hospital-tailored implementation strategy aimed at overcoming the specific barriers to implementation in each participating hospital. Multi-faceted implementation strategies were used, which provided insight, induced change and acceptance, and gave feedback to maintain changes [22]. As a result of the implementation project, breast cancer surgery care became more structured and cost-effective. A recent quantitative sustainability evaluation showed that the early post-implementation results of SSP for breast cancer surgery were maintained in terms of the proportion of patients treated in short stay and adherence to key recommendations to facilitate short stay, five years after the completion of implementation [23].

\section{Hospitals}

For the current study, we selected 14 hospitals from the primary implementation projects. The selection was based on the criterion that the primary implementation strategy had shown early post-implementation effectiveness in these hospitals, in order to increase the chances of finding information on sustainability and its determinants. In the ERAS case, ten hospitals were selected out of the 33 that participated in the primary implementation project. These ten hospitals were identified as implementation champions regarding the ERAS protocol [14] and included seven teaching and three nonteaching hospitals. Hospitals participating in the SSP case were all four hospitals which had successfully participated in the primary implementation strategy, covering the four main organisational types of hospital settings in the Netherlands: one university hospital, two teaching hospitals and one non-teaching hospital. The hospitals are geographically spread over several Dutch regions.

\section{Respondents and sampling}

Respondents were key members of the current multidisciplinary teams. 23 of the 26 respondents had been involved in the initial implementation process, while three respondents had started working after the implementation had ended. Sampling started with the surgeons involved at each location. Surgeons were assumed to know most about the determinants of the sustainability of the programmes, as they were the formal implementation project leaders. In two hospitals, the relevant surgeon was not able to participate in this study, so the first interview was held with a nurse practitioner. Additional respondents were selected by means of snowball sampling. After the first 
interview, the interviewee was asked to propose another possible respondent from their team. For the ERAS case, these respondents were four nurse practitioners and one ward nurse, while one interview was held with a head nurse and a ward nurse together. For the SSP case, the respondents were two breast care nurses and two nurse practitioners.

\section{Data collection}

\section{Semi-structured interviews}

A total of 21 interviews with 26 persons were held, 14 for the ERAS case and seven for the SSP case. Four of the 21 interviews were combined interviews with two or three respondents. Each interview was conducted by one of the two main researchers (FG and SA) and took place in a quiet setting at the hospital.

The content of the semi-structured interview guide (figure 1) was guided by the CFIR. The interview guide was discussed in research team meetings during the data collection process but this did not influence the content of the guide. The main focus of the interviews was on reasons why outcomes and programme elements in both cases were maintained after the initial successful implementation. Hospital specific quantitative results $[21,23]$ were used as a reference for the actual performance during the interviews by presenting and discussing the pre-implementation, implementation and late post-implementation results to key persons. For the ERAS case, hospital specific functional recovery, length of hospital stay and protocol adherence results were presented. For the SSP case, hospital specific results with respect to the proportion of patients treated in short stay and adherence to the key recommendations to facilitate short stay were presented. The two main researchers evaluated the interview guide after the first five interviews had been held in the ERAS case and after three interviews in the SSP case.

The average duration of the interviews was 42 minutes (minimum 19 minutes, maximum 68 minutes). Data were collected between March 2012 and May 2013. All interviews were audiotaped and fully transcribed, and field notes were taken.

\section{Data analysis}

A direct content analysis was performed for the interpretation of the interview data [24]. Interviews were coded per case after which the data were integrated in one database (using NVIVO 10 software). Data collection and analysis were performed simultaneously. The first five interviews were used for a broad analysis of the data, for orientation purposes and were included in the main analyses. Further interviews were analysed using a deductive thematic coding approach for each of the two implementation cases, using the CFIR as a coding scheme. After the codes had been assigned to the various CFIR domains, an inductive coding approach was used at the construct level, to explore emerging themes which were not covered by the constructs but were nevertheless 
regarded as relevant. Data saturation was reached after 18 interviews, and no new data were found in the last three additional interviews.

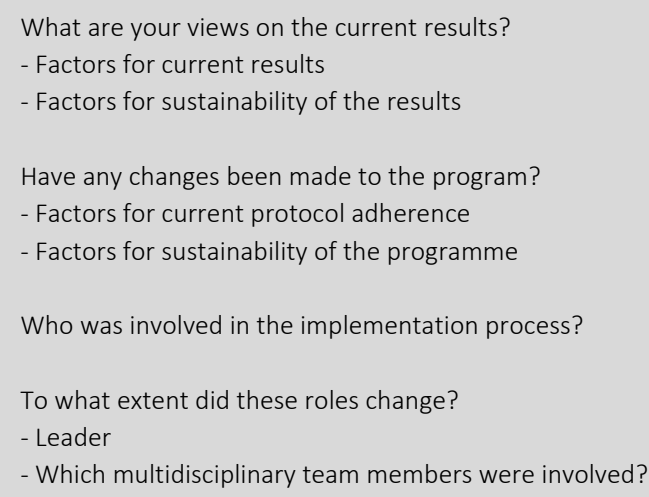

Figure 1. Interview guide

\section{Trustworthiness}

The research team met regularly to discuss the interview guide, sampling and summaries of the interviews, as well as the analysis process, codes and positioning of the codes in the different constructs of the CFIR. Agreement on the coding was reached during consensus meetings. An independent expert on qualitative research (AM), not involved in the project, was consulted to review the research process. Methodological triangulation was ensured by the use of multiple methods of data collection such as in-depth interviews and field notes. Data triangulation was achieved by using multiple sources of data, i.e. interviews with different professionals in different settings. A member check was performed by submitting an interview summary to each respondent for approval. Field notes were used during the analyses to get a better sense of the characteristics of the clinical interview setting. 


\section{CHAPTER 7}

\section{Confidentiality}

Before the start of the interview, respondents were informed that the interview data would be treated confidentially, while anonymity was secured by numbering the interviews. The Medical Ethics Committee of the University of Maastricht granted approval, METC 11-4-015.10.

\section{Results}

\section{Determinants of sustainability}

Determinants of sustainability were found in all domains of the CFIR. The results on the specific determinants are presented for each domain using quotes from the raw interview data and are shown in bold in figure 2. Data which did not fit into the CFIR were classified as determinants beyond CFIR, and are shown in capitals in figure 2 .

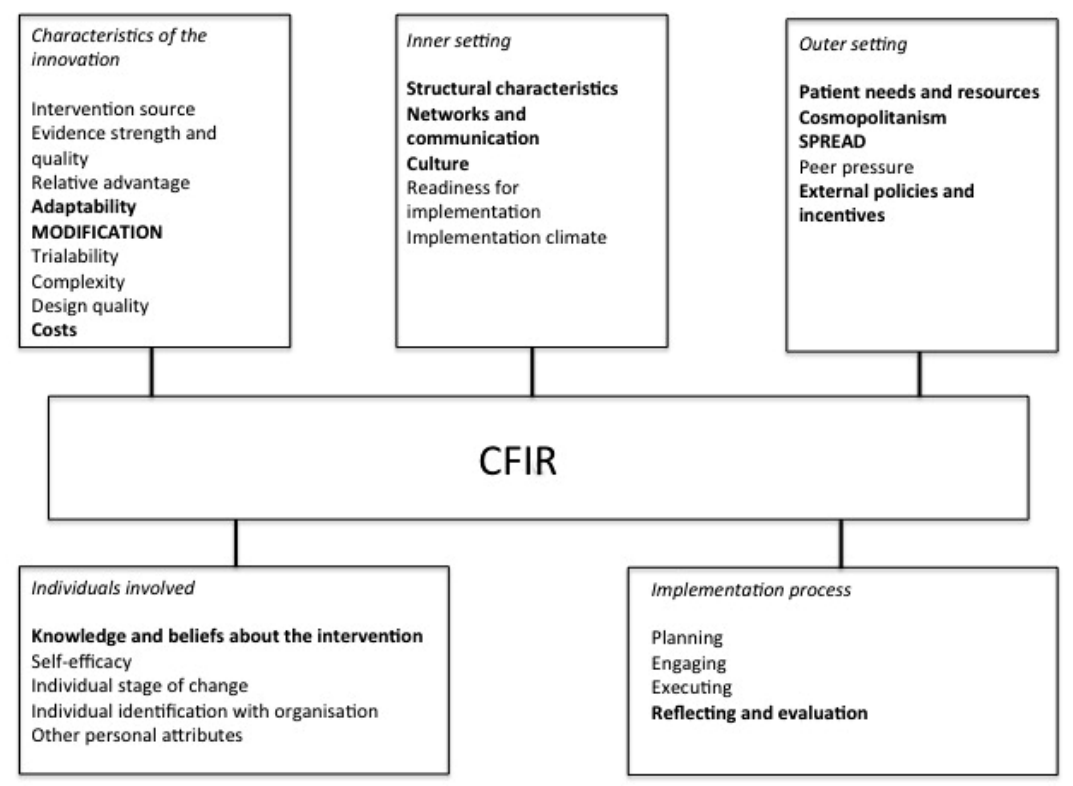

Figure 2. Determinants of sustainability of two multidisciplinary hospital-based programmes after early im plementation success, categorised according to the CFIR. The constructs that were confirmed as determinants of sustainability are printed in bold. Data which did not fit into the CFIR are classified in constructs beyond the CFIR and printed in capitals 


\begin{abstract}
Adaptability
Respondents in both cases mentioned the importance of the possibility to adapt the programme elements to local and patient needs. As a result of this possibility to adapt both programmes, some variation was observed between the participating hospitals. For example, in the SSP case, respondents in one hospital mentioned that they always planned an overnight stay for every patient, while respondents in another hospital customised admission plans for each individual patient, focusing on whether the patient could be treated in day care surgery or required an overnight stay.
\end{abstract}

\title{
Determinant beyond CFIR: Modification of the programme over the years
}

The possibility to modify the programme was also mentioned as a determinant of sustainability. Integration of newly available evidence into the baseline programmes led to modification of the programmes, which helped to sustain them. In both cases new insights and evidence became available during the post-implementation phase. Surgeons in both cases suggested that the programmes had been successfully modified as a result of long-term experience and the emergence of new evidence. We define adaptations to a programme as minor changes within the programme itself to fit local or patient needs, while modifications to the protocol are bigger changes to the protocol itself.

'And then you get the whole analgesics story. There's an overuse of NSAIDs. There have been a number of papers that show that NSAID use leads to more anastomotic leakages. I'll show you a few. This is an article in the British Journal of Surgery from last year... That's a coincidence that I have that here, by the way, it's about a case series from regions near here that shows this. So then we have to get together again and discuss this. So they do get them, but the point is whether we need to change that. This is hard data, you see' (Surgeon, ERAS)

\section{Costs}

Some respondents in the ERAS case noted an economic advantage of early discharge. Respondents from the SSP case mentioned that the proven early post-implementation cost-effectiveness of the programme was a determinant of sustainability. One respondent commented, however, that although it is cost-effective for the hospital, SSP may be a (financial) burden for patients due to more follow-up visits.

I: OK. Day care surgery still works here. But why does it still work? Why is it being implemented so well here? R. Because it's cost-effective as well... And it works; you shouldn't keep people in the hospital longer. That's what makes care expensive' (Nurse Practitioner, SSP) 


\section{CHAPTER 7}

Inner Setting

\section{Structural characteristics}

In both cases, structural characteristics were perceived to be a determinant of sustainability. Low turnover of staff was associated with more knowledge about and experience with the programmes being available than in teams with a higher turnover. Respondents mentioned that new employees have different routines, and perceived this as a barrier to sustainability of knowledge about and attitude towards the programmes. It is especially in teaching hospitals that knowledge of and experience with the programme is lost in the institution, as new doctors enter training every year.

'New people who come in aren't necessarily aware of ERAS, and it's not sustained in such a way that it will continue on its own. People have been trained in different ways and you cannot change that by simply saying 'we do things differently here' (Surgeon, ERAS)

Planning surgery in the early morning was perceived as a determinant of sustainability for both cases. Performing surgery in the afternoon decreases the adherence to protocol elements on the day of surgery in the ERAS case, and decreases the proportion of patients treated in short stay in the SSP case. Lack of integrated planning of staff, operating rooms and beds at institutional level was pointed out as impeding the sustainability of both programmes. Another structural characteristic was that of delegating planning responsibility to one of the professionals in the team. Three to six years after the completion of implementation, those involved in the SSP case perceived the nurses as the coordinators of the care process in most hospitals. They are the case managers for the patients and are responsible for planning and for communication with the patients during the whole care process.

'No, I think the most important reason why this succeeded is due, in our hospital especially, to what I told you before, to the breast care nurse and the specialist nurse. The most important aspect of sustaining it is that the person who is really doing it, and that's not the doctors but the breast care nurses, have a mandate to arrange things. They need to support the idea. It's often 2-3 people and they're not the doctors' (Surgeon, SSP)

\section{Networks and communication}

Short formal and informal communication lines were perceived to be a determinant of sustainability in both cases. Surgeons indicated that short communication lines enabled more control over the performance. In the ERAS case, respondents in some hospitals experienced isolation and differences of interests between disciplines, for instance between surgeons and anesthesiologists, while in other hospitals there were regular meetings with all professionals involved. Open communication and discussions of possi- 
ble shortcomings of a programme among different disciplines were perceived as a determinant of programme adherence in the post-implementation phase. Both programmes were characterised as resulting from multi-disciplinary teamwork. The multidisciplinary character of the programmes was also mentioned as a barrier to sustainability, however, as it complicated communication and reaching a shared vision.

'The multi-disciplinary character makes it really vulnerable, as does its dependence on groups of people who have to keep on doing it, really' (Surgeon, ERAS)

\section{Culture}

Patient satisfaction and high standards for quality of care were viewed as highly relevant in the professionals' value system. However, the ambition to sustain early postimplementation successes varied between hospitals. Some hospitals wanted to maintain their initial results, while others accepted results that were comparable to national results. Culture was perceived to differ between wards. Surgery was perceived as a discipline that is relatively more open to trying out innovations than other disciplines. For example, one respondent indicated that their ward was viewed as 'the' early adopter or pilot ward in the hospital.

'We are the department where all pilot projects that take place in the hospital, they're all run in this department. I really think that has to do with the dynamics of our department, with the group... I guess, I think so. I find it difficult to put this into words for you. I think that it's really kind of part of the nature of being a real department of surgery. ... I think it's also the culture that we have a strong culture of change, and that we, we're really a dynamic department' (Physician assistant, ERAS)

\section{Outer Setting}

\section{Patient needs and resources}

Patient satisfaction was mentioned as determining sustainability of the programmes and was considered to be more important than strictly adhering to the protocol. Respondents in both cases valued client-centeredness, with attention for patient needs and patient satisfaction, as one of the most important focuses in health care.

'I believe that if you want to reach $100 \%$ compliance, you'll have to start pushing people. Because of course, it's possible... Some of these patients would indeed go home, but patient satisfaction will go down. Because a patient who goes home, while feeling that she's not quite ready for it, will give a lower score' (Breast Care Nurse, SSP)

In the ERAS case, respondents mentioned that if a patient does not want to or does not seem to be able to be mobilised or does not want or cannot eat solid foods during the 


\section{CHAPTER 7}

day following surgery, the professionals tended to be less tenacious about this than they had been during the early post-implementation phase.

'I don't want letters of complaint, I want satisfied patients, I don't want to endanger patients etc.; my personal opinion is that that sometimes comes at the expense of the admission day' (Surgeon ERAS)

If patients in the SSP case who were scheduled for day care surgery requested an overnight stay in view of their social context, such an overnight stay was made possible.

'Nowadays we say to people: you decide when you go home, we call that client-friendly' (Surgeon SSP)

\section{Cosmopolitanism}

SSP was reported to be care as usual in the breast cancer wards of many Dutch hospitals nowadays. Communication regarding the programme between hospitals and other health care services was perceived as a determinant of sustainability. Sustainability was facilitated by intensive communication and a strong network connecting different centres. Intensive relations with other centres meant that respondents felt able to share their experiences with the programme.

'So we've known each other for years. You know that the way we work and the protocols have been comparable for years. You know this from the site visits where surgeons from another hospital come and visit us, and where we visit other hospitals in the region. So then you get an impression of how they do it and you know how the breast surgeons work and from this we knew that a lot of the protocols are really similar and that means that breast surgeons have been on a very clear course for some years now' (Surgeon, SSP)

Less intensive communication between hospitals regarding care delivery in the postimplementation phase was mentioned in the ERAS case, and was perceived as a barrier to sustainability by several respondents.

'The implementation goes really well and then it's all up and running and then what? What tricks do others use? See, you read a couple of dissertations on ERAS, but the rest of it you have to deal with completely on your own' (Surgeon, ERAS)

\section{Determinant beyond CFIR: Spread}

Even stronger than external networking with direct peers in other hospitals and sharing experiences with colon cancer and breast cancer teams, is spreading the experiences to professionals in other fields. This spread was perceived as an important determinant of 
sustainability as it was perceived as further confirmation of their effectiveness, and it increased the intention to sustain the care in accordance with these programmes. Respondents from the ERAS case mentioned that, after having gained positive experiences with the ERAS programme for colonic surgery, the programme was spread to other patient groups outside their specialism, e.g. vascular, urological and gynecological surgery departments. Respondents perceived this spread to other disciplines as a facilitator of the sustainability of ERAS programme elements at organisational level. In the SSP case, surgeons from other hospitals approached the early adopters for advice about the implementation of SSP in their setting. Both programmes were spread to other internal or external settings in the post-implementation phase.

'Part of the success is due to the fact that it can be extrapolated to other patient categories. You don't need to think one way in one department and use another policy in another corridor' (Surgeon, ERAS)

\section{External policies \& incentives}

Sustainability of both programmes was perceived to be influenced by external policies. Respondents in both cases referred to their performance being partially audited by means of a national registration database. Several elements of SSP were mentioned as obligatory in the guidelines of the Dutch health care inspectorate (IGZ) which was perceived to impact the sustainability of SSP. The results of these performance measures were felt to create more transparency about performance. However, lack of timely feedback of these national results was said to be a barrier to improving results.

'But OK, we participate in the national registration, the DSCA. So that means that a lot of things are brought out into the open' (NP, ERAS)

\section{Characteristics of individuals}

\section{Knowledge and beliefs}

Knowledge of and beliefs about the programme was perceived as determinant of sustainability. Extensive experience affected the professionals' beliefs regarding the suitability of certain programme elements in particular situations. Some respondents mentioned that some professionals did not adhere to all programme elements and believed that colleagues deviated from the programme because they perceived it as additional workload. Another point about the knowledge and beliefs about the programmes that was mentioned is that knowledge of the results in terms of patient outcomes affects the perception of a programme. Respondents' perceptions of the SSP benefits were positive as a result of long-term experience with the programme. 


\section{CHAPTER 7}

'And that's something that needs to grow on people, and if you see often enough that people are able to go home just fine, and they aren't more likely to come back because of problems, then you start to rely on it a little more yourself'(Breast nurse, SSP)

\section{Implementation process}

The original implementation process, although viewed as being of importance for the early post-implementation results, was not mentioned as having influenced the longterm sustainability of the benefits achieved. Continuous monitoring and feedback of performance and outcomes, which was one of the important components of the Breakthrough Series, was nevertheless viewed as supporting sustainability. Many detailed activities were mentioned, whether stemming from the original list of implementation activities or not, such as feedback and educational meetings. These activities will be reported elsewhere.

'What we did until recently was organise an ERAS lunch every six weeks to three months, where all the patients of the previous period were, not discussed individually, but there was a check to see: Right, what was the length of hospital stay, what's the situation regarding mobilisation, and drinking? That kind of thing, and where does it go wrong?' (Surgeon, ERAS)

\section{Discussion}

The current study explored key determinants of sustainability of two hospital-based multidisciplinary programmes, focusing on sustainability after early post-implementation success was achieved. Perceived key determinants of sustainability were evaluated among relevant professionals three to six years after the completion of successful implementation. The following key determinants of sustainability were perceived: modification and adaptability of the programme, cost-effectiveness, institutionalisation into existing systems, short communication lines within the multidisciplinary team, an innovative culture, benefits to patients, cosmopolitanism, the existence of external policies and incentives, trust and belief in the programme, and spreading the programme to other settings. These determinants of sustainability of the two programmes are partly the same as those proposed by Scheirer [25], and recently by the British NHS [10]. However, external policies and incentives, and spread within and between hospitals were not mentioned in these models but are suggested recently by other authors [11, 26]

External policies and incentives is a broad construct in the CFIR. It includes external strategies from a macro perspective and can be related to for example political or socioeconomic issues [27] or national benchmarking [15]. Over the last years, national registration on cancer care performance to increase quality of care has taken its position in 
the Netherlands. Some key recommendations of SSP were covered as part of national audit systems which was perceived to have influence on the sustainability of SSP. The current study showed that external strategies were perceived to influence the sustainability of the innovations. The determinant spread of the programme within and between hospitals was mentioned also by our respondents. The relation between sustainability and spread has also been reported in the literature [28]. Spread of the programmes within and between hospitals was perceived as further confirmation of their effectiveness, and increased the intention to sustain the care in accordance with these programmes. In terms of the framework for the spreading of innovations proposed by Ovretveit [29], facilitated evolution had happened in the post-implementation phase. There were instances of self-initiated spreading, as a result of long-term experience, proven effectiveness of the programmes and the possibility to tailor and integrate the care programmes to other patient groups or hospitals.

Recent studies have reported that little is known about the influence of innovation modification on the sustainability of innovations [30]. The current study shows that the original programme was being modified in response to patient needs, institutional factors and the emergence of new evidence in the post-implementation phase. For example, in the SSP case, respondents in one hospital mentioned that they always planned an overnight stay for every patient, while in another hospital admission plans were customised on individual patient level. These modifications and adaptations can impact costs, outcomes and experience of the health care innovation. Interestingly, the original implementation process was not perceived to play a major role in the sustainability of the programmes in our study. One reason might be that during the implementation process, little attention was given to sustainability. Although the hospitals were guided by experts during the implementation process, no one focused on long-term results after the original implementation processes had ended. Nevertheless, another study showed that attention to sustainability during the initial implementation process increases the chances of sustainability [10].

\section{Strengths and weaknesses}

A strong point of our study was that we used a qualitative approach to understand the road to sustainability. Exploring perceptions of determinants of success can contribute to a clearer focus regarding the concept of sustainability. The collection of qualitative data and the analysis of determinants of sustainability were guided by the CFIR. This approach was adopted because it is a broad comprehensive model of evidence-based dimensions covering the full context of implementation, and it considers a wide range of possible determinants of sustainability of initial implementation successes. Another strength of our study is the use of quantitative results in the interviews as a reference regarding actual performance, by presenting and discussing pre-implementation, implementation and late post-implementation results with the key persons. This may have helped us to elicit the determinants of sustainability more effectively. Since the inter- 
views were held with professionals from hospitals which had successfully maintained their results, as well as from hospitals with less success in terms of sustainability, they yielded more information about determinants of the sustainability of quality improvement programmes. Hospitals selected for this study where those which had successfully achieved early post-implementation changes. This selection was based on the assumption that early post-implementation success is necessary to achieve sustainability.

A possible weakness is that it could be argued that using the CFIR is not the ideal framework for exploring determinants of sustainability. The CFIR was designed for implementation research rather than for sustainability, so some key determinants mentioned by our respondents were not covered by it. However, the deductive and inductive analyses we used offered enough room to incorporate unexpected findings, allowing us to identify and report major determinants beyond the CFIR. Revealed key determinants which are not part of the CFIR are elements that may not matter in the early post-implementation phase. Only studies focusing on long-term post-implementation evaluation may identify these determinants. Another issue arising from the use of the CFIR is the fragmented approach to the data, as there are no connections between domains in the framework. CFIR is a static model and describes several constructs and factors within these constructs. The dynamics of sustainability may not fit easily in this model, which is why results regarding some determinants overlapped with other determinants.

Most respondents had been actively involved during the implementation project and were expected to have the greatest knowledge about current care. However, information may have been missed as a result of the selection of key persons. Since it were the formal leaders of the care process who were interviewed, they would not refer to themselves regarding leadership. This may have resulted in a possible lack of information about leadership, an important determinant of success in other studies. Also, respondents were self-selected using a snowball sampling method and all respondents were health care practitioners. This may have resulted in selection bias. Relevant perception from hospital staff and staff other than health care professionals (e.g. managers and anesthesiologists) may have been missed. Furthermore, the interviews were not all individual interviews. As a consequence of the attendance of multiple respondents, the content of the answers may be influenced. Another weakness of the study is its small-scale character, which may have influenced the generalisability of the result. Also, the results may have been influenced by the hospital selection which was based on achieving early post-implementation success. Therefore, in other cases and in other fields of health care other determinants of sustainability may emerge.

\section{Future research}

Our findings suggest that the key determinants of sustainability are multidimensional and complex [25]. Unfortunately, sustainability research has focused on a 'one-size-fitsall' approach in different fields of health care [27] and not much research has primarily 
focused on complex multidisciplinary hospital-based innovations. The current study revealed two determinants beyond the CFIR, i.e. modification of the programme over the years and spread of the programme to other contexts. Leadership and the implementation project were not mentioned as having influenced the long-term sustainability of the benefits achieved. More long-term post-implementation evaluations are needed to explore determinants of sustainability which may not be revealed in the current study. Ultimately, future sustainability research may focus on a 'consolidated framework of sustainability research'.

This study showed that some care programme elements had evolved and had been adapted and spread in the post-implementation phase. It is questionable to what degree such changes can still be regarded as adaptations or modifications of the original innovation, or should be regarded as a 'new' innovation. However, it is also questionable if the changes made to the programmes have led to increased fidelity to the programmes in the post-implementation phase. Given the importance of maintaining high quality of care, future research must focus on the nature of modification and spreading of best practices.

\section{Conclusions}

The current study examined the sustainability of two programmes from a broad and exploratory perspective. Modification and adaptability of the programme, institutionalisation into existing systems, short communication lines within the multidisciplinary team, trust and belief in the programme, and spreading of the programme to other settings were perceived to be the key determinants of sustainability of early implementation success. Two of these determinants are not covered by the CFIR, i.e. modification of the programme over the years and spread of the programme to other contexts. This implies that determinants of implementation are different than determinants of sustainability. Furthermore, the determinants of sustainability put forward in both cases were largely the same. The results of this study confirm that sustainability of hospitalbased multidisciplinary programmes is a complex process. The current study showed that sustainability is influenced also beyond the borders of the initial implementation setting, as determinants 'external policies and incentives' and 'spread' were newly revealed determinants of sustainability. Sustainability of innovations is influenced by determinants stemming from all ecological levels of the health care system and needs continuous effort in the post-implementation phase in the form of ongoing quality improvement activities. Implementation teams may consider these determinants when performing quality improvements in health care. 


\section{References}

1. Grol R, Grimshaw J. From best evidence to best practice: effective implementation of change in patients' care. Lancet. 2003 Oct 11;362(9391):1225-30.

2. Hovlid E, Bukve O, Haug K, Aslaksen AB, von Plessen C. Sustainability of healthcare improvement: what can we learn from learning theory? BMC health services research. 2012;12:235.

3. NHS. Sustainability and its relationship with spread and adoption, General improvement skills. Coventry, UK: Improvement Leaders' guide; 2007

4. Duckers ML, Wagner C, Vos L, Groenewegen PP. Understanding organisational development, sustainability, and diffusion of innovations within hospitals participating in a multilevel quality collaborative. Implement Sci. 2011;6:18.

5. Forster DA, Newton M, McLachlan HL, Willis K. Exploring implementation and sustainability of models of care: can theory help? BMC public health. 2011;11 Suppl 5:S8.

6. Gruen RL, Elliott JH, Nolan ML, Lawton PD, Parkhill A, McLaren CJ, et al. Sustainability science: an integrated approach for health-programme planning. Lancet. 2008 Nov 1;372(9649):1579-89.

7. Scheirer M. Is sustainability possible? A review and commentary on empirical studies of program sustainability Am J Eval. 2005;26:320-47.

8. Shediac-Rizkallah MC, Bone LR. Planning for the sustainability of community-based health programs: conceptual frameworks and future directions for research, practice and policy. Health Educ Res. 1998 Mar;13(1):87-108.

9. Ament SMC, Gillissen F, Maessen JMC, Dirksen CD, van der Weijden T, von Meyenfeldt MF. Sustainability of healthcare innovations (SUSHI): long term effects of two implemented surgical care programmes (protocol). BMC health services research. 2012;12:423.

10. Doyle C, Howe C, Woodcock T, Myron R, Phekoo K, McNicholas C, et al. Making change last: applying the NHS institute for innovation and improvement sustainability model to healthcare improvement. Implement Sci. 2013;8(1):127. PubMed PMID: 24160758.

11. Wiltsey Stirman S, Kimberly J, Cook N, Calloway A, Castro F, Charns M. The sustainability of new programs and innovations: a review of the empirical literature and recommendations for future research. Implement Sci. 2012;7:17.

12. Knops AM, Storm-Versloot MN, Mank AP, Ubbink DT, Vermeulen H, Bossuyt PM, et al. Factors influencing long-term adherence to two previously implemented hospital guidelines. International journal for quality in health care : journal of the International Society for Quality in Health Care / ISQua. 2010 Oct;22(5):421-9.

13. de Kok M, van der Weijden T, Kessels A, Dirksen C, van de Velde C, Roukema J, et al. Implementation of an ultra-short-stay program after breast cancer surgery in four hospitals: perceived barriers and facilitators. World J Surg. 2008 Dec;32(12):2541-8.

14. Gillissen F, Hoff C, Maessen JMC, Winkens B, Teeuwen JH, von Meyenfeldt MF, et al. Structured synchronous implementation of an enhanced recovery program in elective colonic surgery in 33 hospitals in The Netherlands. World J Surg. 2013 May;37(5):1082-93.

15. Damschroder $\mathrm{L}$, Aron DC, Keith RE, Kirsh SR, Alexander JA, Lowery JC. Fostering implementation of health services research findings into practice: a consolidated framework for advancing implementation science. Implement Sci. 2009;4:50.

16. Damschroder $\sqcup$, Hagedorn HJ. A guiding framework and approach for implementation research in substance use disorders treatment. Psychol Addict Behav. Jun;25(2):194-205.

17. Kehlet $\mathrm{H}$, Mogensen $\mathrm{T}$. Hospital stay of 2 days after open sigmoidectomy with a multimodal rehabilitation programme. Br J Surg. 1999 Feb;86(2):227-30.

18. Basse L, Hjort Jakobsen D, Billesbolle P, Werner M, Kehlet $\mathrm{H}$. A clinical pathway to accelerate recovery after colonic resection. Ann Surg. 2000 Jul;232(1):51-7.

19. Kilo CM. A framework for collaborative improvement: lessons from the Institute for Healthcare Improvement's Breakthrough Series. Qual Manag Health Care. 1998 Sep;6(4):1-13.

20. Schouten LM, Hulscher ME, van Everdingen JJ, Huijsman R, Grol RP. Evidence for the impact of quality improvement collaboratives: systematic review. Bmj. 2008 Jun 28;336(7659):1491-4. 
21. Gillissen F, Ament SMC, Maessen JMC, Dejong CH, Dirksen CD, van der Weijden T, et al. Sustainability of an Enhanced Recovery After Surgery Program (ERAS) in Colonic Surgery. World journal of surgery. 2014 Aug 23.

22. de Kok M, Frotscher CN, van der Weijden T, Kessels AG, Dirksen CD, van de Velde CJ, et al. Introduction of a breast cancer care programme including ultra short hospital stay in 4 early adopter centres: framework for an implementation study. BMC Cancer. 2007;7:117.

23. Ament SMC, Gillissen F, Maessen JMC, Dirksen CD, Bell AV, Vissers YL, et al. Sustainability of short stay after breast cancer surgery in early adopter hospitals. Breast (Edinburgh, Scotland). 2014 Mar 31.

24. Pope C, Mays, N. Qualitative Research in Health Care: Blackwell Publishing; 2006.

25. Scheirer MA. Is Sustainability Possible? A Review and Commentary on Empirical Studies of Program Sustainability. American Journal of Evaluation. 2005 September 2005;26(3):27.

26. Chambers D, Glasgow R, Stange K. The dynamic sustainability framework: addressing the paradox of sustainment amid ongoing change. Implementation Science. 2013;8(1):117.

27. Greenhalgh T, Robert G, Macfarlane F, Bate P, Kyriakidou O. Diffusion of innovations in service organizations: systematic review and recommendations. Milbank Q. 2004;82(4):581-629.

28. Slaghuis SS, Strating MM, Bal RA, Nieboer AP. A measurement instrument for spread of quality improvement in healthcare. International journal for quality in health care : journal of the International Society for Quality in Health Care / ISQua. 2013 Apr;25(2):125-31.

29. Ovretveit J. Widespread focused improvement: lessons from international health for spreading specific improvements to health services in high-income countries. International Journal for Quality in Health Care. 2011 June 1, 2011;23(3):239-46.

30. Stirman SW, Miller CJ, Toder K, Calloway A. Development of a framework and coding system for modifications and adaptations of evidence-based interventions. Implement Sci. 2013;8:65. 



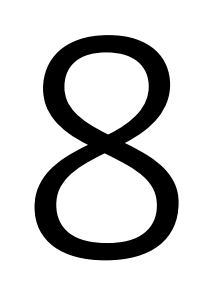

\section{Identification of promising strategies to sustain improvements in hospital practice:} a qualitative case study 


\section{CHAPTER 8}

\section{Abstract}

\section{Background}

A quality improvement collaborative is an intensive project involving a combination of implementation strategies applied in a limited "breakthrough" time window. After an implementation project, it is generally difficult to sustain its success. In the current study, sustainability was described as maintaining an implemented innovation and its benefits over a longer period of time after the implementation project has ended. The aim of the study was to explore potentially promising strategies for sustaining the Enhanced Recovery After Surgery (ERAS) programme in colonic surgery as perceived by professionals, three to six years after the hospital had successfully finished a quality improvement collaborative.

\section{Methods}

A qualitative case study was performed to identify promising strategies to sustain key outcome variables related to the ERAS programme in terms of adherence, time needed for functional recovery (FR) and hospital length of stay (LOS), as achieved immediately after implementation. Ten hospitals were selected which had successfully implemented the ERAS programme in colonic surgery (2006-2009), with success defined as a median LOS of six days or less and protocol adherence rates above $70 \%$. Fourteen semistructured interviews were held with eighteen key participants of the care process three to six years after implementation, starting with the project leader in every hospital. The interviews started by confronting them with the level of sustained implementation results. A direct content analysis with an inductive coding approach was used to identify promising strategies. The mean duration of the interviews was 37 minutes (min 26 minutes - max 51 minutes).

\section{Results}

The current study revealed strategies targeting professionals and the organisation. They comprised internal audit and feedback on outcomes, small-scale educational booster meetings, reminders, changing the physical structure of the organisation, changing the care process, making work agreements and delegating responsibility, and involving a coordinator. A multifaceted self-driven promising strategy was applied in most hospitals, and in most hospitals promising strategies were suggested to sustain the ERAS programme.

\section{Conclusions}

Joining a quality improvement collaborative may not be enough to achieve long-term normalisation of transformed care, and additional investments may be needed. The findings suggest that certain post-implementation strategies are valuable in sustaining implementation successes achieved after joining a quality improvement collaborative. 


\section{Background}

Starting a quality improvement collaborative (QIC) is a widely used implementation strategy, with the intention to bring about large-scale change in health care [1, 2]. A QIC is an intensive project with a combination of implementation activities executed in a limited 'breakthrough' time window and applied in multidisciplinary teams. Despite the frequent application of QICS, the quality improvements achieved may not be sustained in daily practice, as the implementation strategy may not have a long-term effect [3-5]. It is only recently that sustainability of health care innovations has gained attention in implementation science, so the concept of sustainability is not well defined yet $[4,6,7]$. Most implementation projects have focused on short-term results, and sustainability research is performed in various manners. The current study used the following definition of sustainability: "sustainability of change exists when a newly implemented innovation continues to deliver the achieved benefits over a longer period of time [8] and definitely does not return to the previous processes [3], even after the implementation project is no longer actively carried out" [9]. Unfortunately, sustainability of quality improvement is not self-evident, as it is a dynamic and complex process $[4,6,8]$.

After successful implementation of a health care innovation in practice it is important that the results achieved are sustained to prevent a waste of implementation efforts and costs and to prevent suboptimal care delivery by professionals who fall back on old routines [10]. Recently, Stirman et al. presented four main determinants of sustainability, viz. innovation characteristics, context, capacity and processes and interactions [6]. In the processes and interactions determinant, they included activities such as evaluation and feedback, shared decision making among stakeholders, integration of rules and training and education. Doyle et al. proposed factors that may affect the sustainability, including monitoring performance and organising educational activities [11] Thus, additional activities after the completion of an implementation project such as joining a QIC may be valuable for the sustainability of innovations.

Between 2006 and 2009 a QIC (breakthrough implementation strategy) was used to implement the Enhanced Recovery After Surgery (ERAS) programme in colonic surgery (figure 1). An ERAS programme is an evidence-based perioperative care protocol which leads to faster functional recovery [12-17] and is associated with earlier hospital discharge and reduced hospital costs [18-20]. Implementation results showed a decrease in time to functional recovery, a decrease in hospital length of stay and increased adherence to the ERAS programme elements [21]. The ERAS programme is currently being introduced to other surgical fields [22, 23]. However, recent research showed variability in the long-term impact of the QIC with respect to the professionals' adherence to the innovation and key outcomes (functional recovery and hospital length of stay) between hospitals that had succeeded in implementing the ERAS programme in colonic surgery [24]. Subsequent research identified the determinants of the sustainability of adherence to the ERAS programme elements and the related benefits (What 


\section{CHAPTER 8}

determines sustainability of two quality improvement programs after achieving early implementation success? A qualitative case study, Chapter 7). This generated important insights into the role of specific determinants of sustainability. Apart from various innovation and contextual determinants, it became clear that active post-implementation strategies may play a role in sustaining the successes achieved.

The aim of this study was to explore promising post-implementation hospital specific strategies as perceived by professionals to maintain or improve primary implementation successes (the innovation and its benefits). For this purpose, we identified the professionals' ideas and insights regarding the post-implementation activities they perceived to be promising for sustaining the adherence to the ERAS programme and its benefits achieved. Not much research has been done regarding the sustainability of changes after a hospital has successfully finished a QIC [1]. Moreover, there have been calls for follow-up implementation studies to achieve a better understanding of longterm improvement processes [8].

Breakthrough series in three groups of hositals $(n=33)$ to implement the ERAS programme in colonic surgery

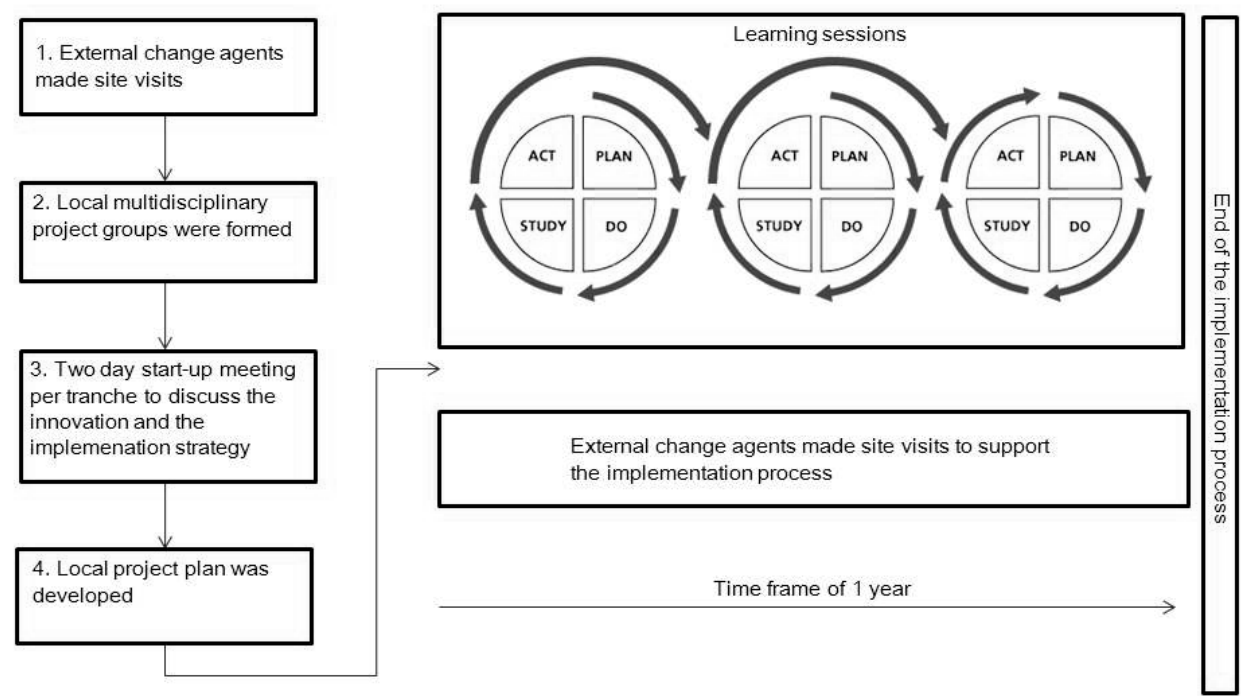

Figure 1. The implementation process of the ERAS programme using Breakthrough Series between 2006-2009 


\section{Methods}

\section{Study design}

A qualitative single-case study was conducted to identify promising strategies to sustain achieved successes as perceived by health care professionals after the hospital had joined a QIC. A case study can be used to explore "how"- and "why"-type research questions about contemporary phenomena [25]. An instrumental case study can be conducted to examine a phenomenon using a typical case $[26,27]$. Since the aim of the study was to explore promising post-implementation strategies to sustain health care innovations, we opted for an instrumental case study approach. Our case study involved the sustainability of the ERAS programme in colonic surgery after the hospital had joined a QIC. The rational for a single-case study design was the combination of a critical and a longitudinal case which according to Yin [25] are elements for single-case designs. The case is critical because of the early post-implementation success and the shared implementation experience. The case is longitudinal as we studied the ERAS programme at several points in time.

\section{Research project}

The present study is part of a research project, the sustainability of health care innovations (SUSHI) study [9]. The overall objective of this research project was to explore the concept of sustainability of two surgical innovations. One of the surgical innovations was the ERAS programme in colonic surgery. As shown in previous research, structural methods for sustainability evaluations are lacking $[6,28]$. Also, there is no standard timeframe for evaluating sustainability yet. The SUSHI study used a time frame of three to six years after finishing the primary implementation process.

The primary implementation process (breakthrough implementation strategy) of the ERAS programme was externally guided by the Dutch Institute For Healthcare Improvement (CBO). Hospitals participating in the SUSHI study were selected from the 33 Dutch hospitals that initially participated in the primary implementation strategy. In the SUSHI study, hospital selection was based on the criterion that the implementation strategy had been successfully applied in these hospitals, in order to increase the chances of finding information on sustainability and its determinants. Implementation success of the ERAS programme was defined as achieving (1) a median hospital length of stay of six days or less of patients undergoing colonic surgery, (2) an overall protocol adherence to the ERAS programme above 70\%, and (3) at least 40 patients treated within the year of the implementation project [21]. Ten of the 33 hospitals that had initially participated in the primary implementation strategy met the inclusion criteria for the SUSHI study.

The sustainability of the ERAS programme was evaluated three to six years after implementation (late post-implementation measurement, 2012) and compared with the performance at the end of the implementation project (early post-implementation 
measurement, 2006-2009). As part of the SUSHI study, the sustainability of the ERAS programme for colonic surgery was analysed by means of quantitative and qualitative methods. The quantitative sustainability evaluation focused on two key outcome variables: the level of professionals' adherence to the ERAS programme elements, and key outcomes at the patient level [21]. Professionals' adherence was defined as the proportion of patients receiving care according to the ERAS programme. Key outcomes at patient level were time needed for functional recovery and hospital length of stay. Functional recovery was defined as adequate pain control requiring oral analgesia only, sufficient oral intake, and independent mobility sufficient to perform activities of daily living at the preoperative level. Hospital length of stay was defined as the number of nights in hospital after surgery.

The sustainability of the ERAS programme was assessed on key outcome level. The results of the quantitative sustainability study were that:

- the implementation successes in protocol adherence was not acceptably sustained, as, in most hospitals, adherence to the ERAS programme was lower in the late postimplementation measurement compared with the early post-implementation measurement,

- time to recovery was acceptably sustained in most hospitals

- the reduction in LOS was not fully sustained in all hospitals.

- the study showed that patients were significantly older and physically more complex, and that the proportion of patients operated with laparoscopic surgery increased in the late post-implementation measurement compared with the early post-implementation measurement.

These results suggested that a dynamic context may had occurred that could influence the sustainability of the ERAS programme. Subsequently, a qualitative study was carried out and identified the determinants of the sustainability of adherence to the ERAS programme elements and the related benefits. Key results were that the sustainability was influenced by:

- Modification and adaptability of the programme,

- institutionalisation into existing systems

- short communication lines within the multidisciplinary team

- trust and belief in the programme, and

- spreading of the programme to other settings (What determines sustainability of two quality improvement programs after achieving early implementation success? A qualitative case study, Chapter 7).

The results of the previous studies related to the SUSHI study focused on the level of sustainability of outcomes such as professional adherence, patient key outcomes and on the determinants of sustainability. In this study we turn our attention to another unit of sustainability analysis: strategies applied to facilitate the sustainability of the ERAS programme after the hospital had successfully finished a quality improvement collabo- 
rative. Promising strategies for the sustainability of the ERAS programme were explored in relation to the professionals' adherence to the programme elements, and time needed for functional recovery and hospital length of stay [24].

The case being studied in the current study was defined as 'the event of successful early post-implementation of the ERAS programme using a quality improvement collaborative'. The first boundary of the current case study was that, the combination of the implementation strategy and the innovation used, influences the level of quality improvement [29]. The hospitals in this study shared the experience of joining a quality improvement collaborative to implement the ERAS programme for colonic surgery between 2006 and 2009. The second boundary of the current case study was the delivery of colonic surgery care in a multidisciplinary setting in the Netherlands. The third boundary of the case study was the focus on the ten hospitals that showed early postimplementation success while 23 out of 33 hospitals did not achieve this result. The fourth boundary of this case study was the post-implementation phase. What lies beyond the boundaries were the 23 out of 33 hospitals that did not achieve early postimplementation success.

\section{Theoretical orientation}

The present study used the Cochrane Effective Practice and Organisation of Care (EPOC) Data Collection Checklist $[29,30]$ as a theoretical orientation. The EPOC checklist was developed by the EPOC group and is a resource to support researchers and reviewers in conducting and assessing systematic reviews about interventions, to improve the delivery, practice and organisation of health care services. The EPOC checklist contains a taxonomy of implementation activities, called "types of interventions". Implementation activities are classified into professional interventions, financial interventions, organisational interventions and regulatory interventions. The taxonomy as described by the EPOC group was used as a reference point to identify and analyse relevant data.

\section{Respondents and sampling}

\section{Hospitals}

Hospitals that had implemented the ERAS programme successfully after finishing the QIC [24] were included. These were the same hospitals as included in the SUSHI study, and were found to differ in the level of sustaining the primary implementation success (table 1). The hospital selection included seven teaching and three non-teaching hospitals.

\section{Respondents}

In total, ten gastrointestinal surgeons, two physician assistants, two coordinators and four nurses were interviewed between March 2012 and February 2013. Formal project leaders were purposively sampled at hospital level as they were expected to have the 


\section{CHAPTER 8}

best knowledge about possible sustainability strategies. In each hospital, the formal project leader was a surgeon, and they were interviewed first. In nine of the ten participating hospitals, it was the formal project leader who was interviewed while in one of the ten hospitals, a physician assistant was interviewed instead of the formal project leader, who lacked the time to participate. Sixteen of the eighteen respondents had been involved in the implementation of the ERAS programme, while two physician assistants had been appointed in the post-implementation phase and were currently key persons in the care process related to the ERAS programme in colonic surgery. A snowball sampling method was used to identify and recruit other respondents. Characteristics of the respondents are presented in table 2.

Table 1. Early post- and late post-implementation results of the ERAS programme for each hospital

\begin{tabular}{|c|c|c|c|c|c|c|}
\hline & \multicolumn{2}{|c|}{ Hospital length of stay } & \multicolumn{2}{|c|}{ Time to functional recovery } & \multicolumn{2}{|c|}{$\begin{array}{l}\text { Adherence to the ERAS } \\
\text { programme }\end{array}$} \\
\hline & Impl & Post-impl & Impl & Post-impl & Impl & Post-impl \\
\hline Hospital 1 & 6 & 7 & 3 & 3 & 77 & 61 \\
\hline Hospital 2 & 4 & 8 & 3 & 4 & 82 & 70 \\
\hline Hospital 3 & 5 & 5 & 3 & 2 & 71 & 56 \\
\hline Hospital 4 & 5 & 6 & NA & 3 & 65 & 68 \\
\hline Hospital 5 & 6 & 6.5 & 3 & 3 & 78 & 60 \\
\hline Hospital 6 & 4 & 6 & 3 & 3 & 87 & 69 \\
\hline Hospital 7 & 5 & 7.5 & 3 & 3 & 80 & 73 \\
\hline Hospital 8 & 5.5 & 6 & 3 & 2.5 & 75 & 68 \\
\hline Hospital 9 & 6 & 5 & 4 & 3 & 68 & 70 \\
\hline Hospital 10 & 6 & 5 & 4 & 3 & 64 & 72 \\
\hline
\end{tabular}

NA: Not Available

Data on length of stay and time needed for functional recovery are median numbers of days. Data on compliance are percentages. Data of compliance are percentages

\section{Data collection}

\section{Semi-structured interviews}

Data was collected by means of semi-structured interviews three to six years following implementation. In two of the ten hospitals, the interview sessions were attended by two and three respondents simultaneously. The main focus of the interviews was whether they perceived that outcomes and elements of the ERAS programme were maintained after the initial successful implementation, and how they could have been or were maintained. Respondents were asked to retrospectively reflect on the years following implementation in terms of the supportive actions that had been undertaken by the hospital and were perceived to be promising in sustaining achieved outcomes in 
terms of length of stay, time needed for functional recovery, and adherence to the ERAS programme elements. The interviews were transcribed and field notes were taken. The mean duration of the interviews was 37 minutes (min 26 minutes - max 51 minutes). A member check was performed by submitting an interview summary to each respondent for approval. The data saturation level was reached after 14 interview sessions with 18 interviewees.

Table 2. Respondent and interview characteristics for each session

\begin{tabular}{|c|c|c|c|}
\hline $\begin{array}{l}\text { Interview session } \\
(n=14)\end{array}$ & $\begin{array}{l}\text { Respondent } \\
(n=18)\end{array}$ & Function & Sex \\
\hline 1 & Hospital 1 (n=1) & Surgeon & M \\
\hline 2 & Hospital 1 (n=1) & Nurse & M \\
\hline 3 & Hospital 2 (n=1) & Surgeon & M \\
\hline 4 & Hospital 3 (n=1) & Surgeon & $\mathrm{M}$ \\
\hline 5 & Hospital 3 (n=1) & Unit coordinator & $\mathrm{F}$ \\
\hline 6 & Hospital 4 (n=1) & Surgeon & M \\
\hline 7 & Hospital 5 (n=1) & Surgeon & M \\
\hline 8 & Hospital $6(n=1)$ & Surgeon & M \\
\hline 9 & Hospital 6 (n=3) & Unit coordinator, 2 nurses & $F, F, F$ \\
\hline 10 & Hospital 7 (n=1) & Surgeon & M \\
\hline 11 & Hospital 7 (n=1) & Nurse & $\mathrm{F}$ \\
\hline 12 & Hospital $8(n=2)$ & 2 Surgeons & $\mathrm{M}, \mathrm{M}$ \\
\hline 13 & Hospital $9(n=2)$ & Surgeon, Physician assistant & $F, M$ \\
\hline 14 & Hospital $10(n=1)$ & Physician assistant & $\mathrm{F}$ \\
\hline
\end{tabular}

\section{Data analysis}

A directed content analysis with an inductive coding approach was used to identify promising strategies to sustain the quality improvements achieved [31,32]. Implementation strategies as described by the EPOC group were used deductively as a guiding analytical framework to identify and analyse relevant data. At the same time we also worked inductively to leave room for data that were not covered by the EPOC checklist but were relevant to answer the research question. Interviews were coded independently by SA or FG and one of the other authors. Text fragments referring to activities aimed at sustaining or improving the quality improvements achieved in the postimplementation phase were identified and coded. We used a sensitizing concept approach in which concepts are being used to guide the analysis process [33]. Codes were discussed during consensus meetings with project group members. The data were analysed using NVivo research software version 10. The interviews were performed, fully transcribed and analysed in Dutch language. For publishing purposes, the quotes and codes used in the current paper were translated by a professional translator. 


\section{CHAPTER 8}

\section{Trustworthiness}

To prove trustworthiness two criteria should be met: credibility and transferability [34]. Credibility is concerned with the aspect of truth value. To establish credibility the strategies of triangulation and member check have been applied. Triangulation is the use of evidence from different methods, investigators and sources. To ensure methodological triangulation, data were gathered by means of semi-structured interviews and field notes. Investigator triangulation was assured by involving several investigators as members of the research team. The members of the research team met on a regular basis to reflect on the research process as a whole, the organisation of the project and methodological issues. If consensus was not reached during consensus meeting, a third project group member was consulted (TVW). Regular analysis sessions were held to reflect on the analysis and interpretation of the data. The expertise of investigators were: implementation science, clinical expertise in cancer care and qualitative research. A member check is the feedback of the respondent on the interview transcripts to improve accuracy. All respondents received a summary of the transcripts of the interviews with the request to check for authenticity. In all cases the participants approved the transcripts.

Transferability is concerned with the aspect of applicability [34]. Transferability can be proven by thick description. Descriptive data, such as setting, sample of the hospitals and sample of participants, sample size, sample strategy, inclusion and exclusion criteria, interview procedure and quotes from participants, are provided to enable the reader to make a transferability judgment. A reflective logbook was kept by the researcher to reflect on methodological, analytical and organisational matters related to this study.

\section{Confidentiality}

Before the start of the interview, respondents were informed about the confidentiality of the interview data. Anonymity was secured by numbering the respondents and the interviews. The Medical Ethics Committee of the University of Maastricht had granted approval, METC 11-4-015.10.

\section{Results}

Respondents of all hospitals mentioned promising strategies, targeting both professionals and the organisation, to sustain early implementation successes.

\section{Strategies targeting professionals}

\section{Internal audit and feedback of outcomes}

From a long-term perspective, continuous monitoring and systematic timely communication of the results achieved in the post-implementation phase were perceived by most respondents to encourage staff to sustain behaviour changes and therefore as 
contributing to sustained quality improvement. Respondents perceived monitoring combined with feedback activities as an effective activity to motivate, remind and encourage staff to maintain adherence to the ERAS programme.

"(...) because the project ended. The CBO-project was finished and the database we had been keeping ended and of course it's really intensive work to keep that up. But you do find that you see some things slide. What maybe could have been different, is that we could have monitored for a longer period after it finished. We kept records for a long time of course and at a certain point that stops. (...) I personally prefer to measure things, to measure is to know, I think so. Because it does give you a little bit of focus, clarifying that this is why we're doing this, and this is what we're doing and these are the results and it really provides good insight. So in that sense it could be helpful, I think, yes. (...) But I do believe it is important that there's a kind of post-implementation phase, where you measure, even if it's only once a month or so." (Unit coordinator)

In one hospital, feedback about adherence to the ERAS programme was organised in the format of "ERAS lunches". All respondents mentioned using the output of a national registration database, which was founded in the post-implementation phase, for general performance evaluation. Some respondents said they had an additional internal monitoring system. The aim of such internal audit systems was to identify the determinants of outcomes achieved at their hospital.

"They're interested right away. The funny thing is that you only get that when you show those numbers. And then they really don't mind when it's not going well, as long as you know why it's not going well. That is exactly what you want, then you're going to use those numbers well. See, those half-yearly numbers, they come from now until December, and then next year we'll really start doing that as a standard procedure." (Surgeon)

\section{Reminders}

Some respondents highlighted the importance of administrative reminders integrated in daily practice, such as checklists in patient files, to increase the awareness of and adherence to the ERAS programme. These reminders mainly focused on the nursing activities of the ERAS programme.

"You also see this when we admit the patients. (Respondent picks up a checklist form from a tray), patients we admit from the outpatient department are given an appointment. We've been doing this for four years now, and it's in fact become a regular component, preparing patients for their operation. And afterwards as well. So it's actually completely integrated in the process." (Surgeon) 


\section{CHAPTER 8}

Repeated questioning of non-adherence by colleagues and reminding each other during care delivery were also mentioned as promising activities to increase awareness of and insights into the consequences of actions.

"When you're constantly told "why this", "why that", that you're not doing something correctly, I don't want to call it failing, but there will be an improvement, because you're confronted every time. I think that that's it." - "He was in surgery on Friday, and he was able to go home on Monday. He's still here. He eats, he drinks, he does everything. And why is he here?" (Physician assistant)

\section{Small-scale educational booster meetings}

Respondents perceived investment in ongoing education to maintain and spread evidence-based knowledge as a promising activity in the post-implementation phase.

"Well yes, repetition. See, for me it's obvious, I don't think I need that every time. But I do notice in the team that it's quite important that you keep repeating things, at least on a yearly basis." (Nurse)

Some respondents reported that obligatory educational meetings had been organised to maintain knowledge and to spread new insights in the post-implementation phase. The ERAS programme has been integrated in the educational sessions for existing staff. One respondent explained that yearly education sessions were tailored to the experience and knowledge of different subgroups within the multidisciplinary team, with specific attention for educating new employees.

"With the registrars it's the case that because we have so many staff changes among registrars that you have a new bunch of six registrars that need to receive that training again. So that's pretty much standard, the training for the nurses is adapted as the level of experience rises." (Physician assistant)

Other respondents expressed the view that experiencing the ERAS programme in practice was sufficient to facilitate adherence to the programme. Respondents also indicated that it was common practice to admit ERAS patients to a non-gastrointestinal department, due to limited admission capacity. These patients received care by teams which had not joined the QIC and were therefore not familiar with the ERAS programme elements. As a consequence, some hospitals took the initiative to organise activities to spread the knowledge about the ERAS programme within their hospital.

"We recently trained other surgical departments because we have given up some beds to Gl surgery, because there's a larger number of people ending up there." (Surgeon) 
The ERAS programme comprises elements requiring patient adherence, for example early oral intake and mobilisation. Not all care providers have enough experience of ways to encourage patients to adhere to such protocol elements. Respondents suggested that it is important to instruct staff in how to interact with and motivate patients to adhere to specific guideline recommendations.

"The one making rounds, if all is as it should be, they're trained in such a way that they motivate the patient. And that we'll say to patients 'we have a lot of work to do'." (Physician assistant)

\section{Strategies targeting the organisation}

\section{Changing the physical structure of the organisation}

In some hospitals, certain components of the organisational structure were adapted to facilitate the normalisation of the ERAS programme elements. The ERAS programme influenced the extent to which elective colon patients were clustered in a specific department. Clustering was recognised as a promising activity to increase the feasibility of some ERAS programme elements, such as early mobilisation of patients, in daily practice.

"And it's led to the situation that all intestinal cases go to one section of B2, before they were all mixed together and then the nurses pretty much suggested that the patients should be clustered." (Surgeon)

Respondent: "We really have no unfavourable outcomes, and that's actually a rare thing."

Interviewer: "Perhaps the workload has increased?"

Respondent: "The nursing staff don't think so, which is striking. I thought it would, but they (the nurses) are not perceiving it that way, especially since we allowed them to cluster them (the patients) together in one room. (...) But if you just know that today, or for a couple of days, you've got the fast recovery group, I think they then perceive that as 'right, let's check the list for all four beds." (Surgeon)

One respondent perceived a positive change in the nurses' attitudes towards nurse specific items of the ERAS programme after a shared facility for colon patients had been created, in that collaboration and communication among nurses had improved.

\section{Changing the care process}

Some respondents perceived treatment of patients in batches as a promising activity and highlighted that it seems crucial to invest in discharge planning, although this is not a formal component of ERAS. Respondents emphasised that actual discharge as soon as 


\section{CHAPTER 8}

a patient is found to be recovered and able to go home is still a relevant topic deserving attention. Other respondents mentioned that it would help to have greater uniformity in the procedure for planning and discussing the timing of discharge. Improved organisation, early communication to the patient about discharge and coordination of discharge planning were suggested as promising activities to decrease the late postimplementation length of hospital stay.

"I think that we don't focus as much on that, you always have a home situation that's really a factor that determines whether you stay a little longer or not. If it's all arranged, it's possible. Yes, I believe you need to arrange that well." (Surgeon)

"But the problem is, and that's the way I see it, you have to immediately when the patient is admitted, or really when the patient is referred, you have to start arranging home care and that kind of thing." (Surgeon)

\section{Involving a coordinator}

Investment in staff capacity to sustain improvements related to the key outcome variables was mentioned as a promising activity to sustain early post-implementation success. Some respondents felt a need for a specific coordinator, a task usually delegated to a nurse, who is responsible for systematic checks and monitoring of outcomes and adherence. In cases where late post-implementation successes relapsed, more coordinating efforts were perceived to be necessary.

"We really don't have any key person, where you can say that they're coordinating things at this point. It's kind of been adopted, while nobody really has taken on that key position. And I think there's improvements to be there. When you have someone who keeps on pushing. And that could be the stoma nurse specialist in this case. They see all those patients before and after, and they are in contact with the surgeons, that you could profit from that." (Surgeon)

"The anaesthetist, we noticed very soon that that didn't work too well, so we transferred that to the anaesthesia assistant, that's XXX, but then after a while she started training to become a physician assistant. (...) We also had XX at the department, but I don't know if she, I don't really check that, you see, I should check that, I think it's still being done, but I have to check it, that's the way it works with us humans." (Surgeon)

\section{Making work agreements and delegating responsibility}

Embedding ERAS programme elements in local protocols and performance targets was considered to be a promising activity to improve sustainability. Setting specific outcome targets related to the key outcome variables of the ERAS programme was perceived to facilitate the sustainability of performance. 
"So I think that the way it was set up by the CBO that that's a good way. It has been tried here as well without the $C B O$ at one point, then you see it fail, and then the second time in that structure you see it succeed, and you try it in a way that suits your hospital. And now you find: it's been implemented, everyone is working according to it but now you have to drop some anchors so you can indeed fix a line now and then. And then I say: it's really important to have points of reference." (Nurse)

Respondents mentioned that staff needed to be aware of changes in agreements and responsibilities to get all team members to shift their performance in the same direction. Coordinators and professionals can refer to the guideline if unwarranted deviations from the ERAS programme are noticed. Some key persons delegated part of the responsibility for carrying out ERAS related tasks to other multidisciplinary team members in the post-implementation phase. Shifting responsibilities from initial project leaders to other members of the multidisciplinary team was perceived to increase the chances of sustainability in the post-implementation phase.

"We see in theatre now that it's going really well, so there isn't really a need for action now. Somebody from anaesthesiology has been made responsible for the protocol there, that's another discipline, but it has been made responsible precisely because of that. Because if you keep everything here with you then everyone, now everyone has received their responsibility, that works really well." (Surgeon)

\section{Overview of strategies in the various hospitals}

Table 3 shows that the combination of strategies differed for each hospital. The table gives an overview of the strategies, as identified per hospital, that were actually applied, those that were suggested and those that were not mentioned. Strategies not mentioned are those that were not applied or suggested in one of the participating hospitals, but were identified in other hospitals. Furthermore, most hospitals applied more than one strategy, i.e. a multifaceted self-driven strategy, to increase the chances for the sustainability of ERAS programme related performance in the post-implementation phase. The strategy of 'making work agreements and delegating responsibility' was applied in the largest number of hospitals, while the strategy of changing the care process was suggested in the largest number of hospitals. 
CHAPTER 8

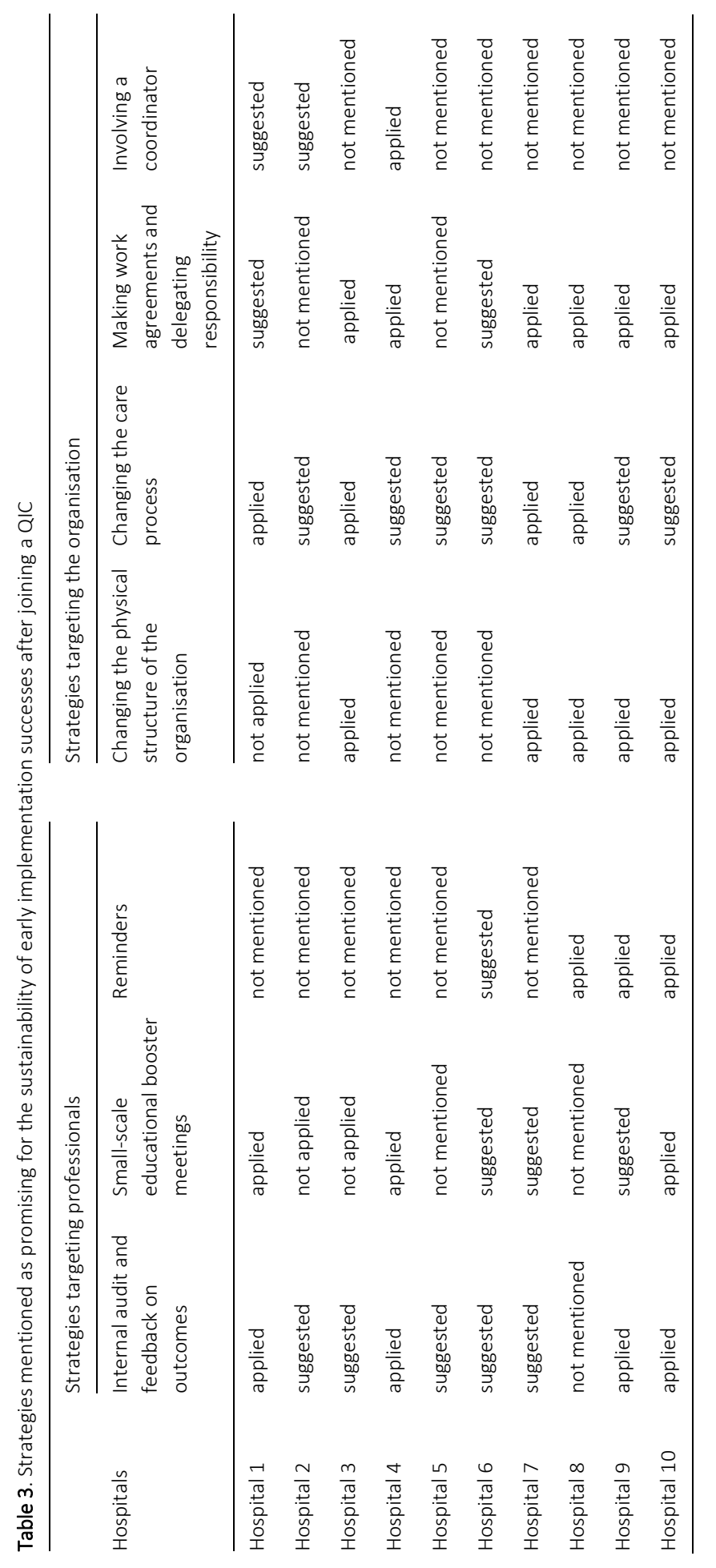




\section{Discussion}

The aim of this study was to explore promising post-implementation hospital-specific strategies to maintain or improve primary implementation successes as perceived by professionals after their hospital joined a QIC. In all hospitals, certain post-implementation strategies, either targeting the professionals or the organisational, were mentioned as promising for the sustainability of initial implementation successes in terms of adherence to the ERAS programme elements, time needed for functional recovery and hospital length of stay. Strategies identified as targeting at the professionals were internal audit and feedback on outcomes, small-scale educational booster meetings and reminders. Strategies identified as targeting the organisation were changing the physical structure of the organisation, changing the care process, making work agreements and delegating responsibility and involving a coordinator.

This late post-implementation evaluation revealed how the target group viewed the possibilities for sustaining the ERAS programme in response to the changing context after the hospital had joined a QIC. In an updated systematic review, Hulscher et al. mentioned that systematic data collection may increase the maintenance and spread of collaborative successes after the initial implementation period has finished [35]. In the current study, professionals indicated also that information about performance is a promising strategy for sustainability. This finding is in line with recent research about the implementation of the ERAS programme using a QIC [36]. This study also suggested continuous measurement of outcomes as an activity to sustain results. Furthermore, the current study found that more structured discharge planning was regarded as a promising activity to improve early post-implementation results of the ERAS programme. However, the primary implementation study [37] had already concluded that the primary implementation strategy failed to cover the organisation of discharge planning. At that time, structured discharge planning was already pointed out as a promising activity to improve the early post-implementation results of the ERAS programme. Unfortunately, after a collaborative project has ended, teams usually do not plan activities to maintain the implementation successes achieved [38]. Nevertheless, the current study showed that some teams used self-initiated strategies to refresh the knowledge about the ERAS programme and even spread it to other departments in their hospital.

The current study confirms that additional bottom-up initiated strategies were perceived to be promising to sustain evidence-based care delivery in the post-implementation phase. Following Pettigrew [39], context 'concerns itself both with influence from the outer context (such as the prevailing economic, social, political environment) and influences internal to the local organisation under study (for example, its resources, capabilities, structure, culture and politics).' Implementation research confirmed that context-related domains, such as the outer setting, the inner setting and the characteristics of individuals, affect implementation of innovations [40]. Institutional differences may result in different perspectives on post-implementation strategies in different hos- 
pitals [41]. Besides differences on institutional level, the external context changed as a consequence of time between the end of the implementation process and the sustainability evaluation. Since 2010, the ERAS society has started to be an international leader in the field of the ERAS programme [42]. One of the aims of the ERAS Society is to continuously review and update the literature and to facilitate in the implementation of the ERAS programme. Also, as mentioned in the context description of the current study, a recent study related to the main research project analysed quantitatively the level of sustainability of the ERAS programme for colonic surgery [24]. This study revealed that patients were significantly older and physically more complex, and that the proportion of patients operated with laparoscopic surgery was higher in the late postimplementation measurement compared with the early post-implementation measurement. These findings imply that too much focus on systematic and fixed postimplementation strategies may limit sustainability and may be a barrier to future innovation. Determinants of sustainability, such as modification of the innovation or changes in the context, may lead to the need for activities to promote the sustainability of the innovation.

One strength of our single-case study approach is the possibility to perform indepth analyses, and the opportunity to analyse and combine data from multiple sources [27]. The broad sample of hospital profiles with respect to organisational characteristics and the variety of late post-implementation successes can potentially enrich implementation research by revealing patterns of variation in executed and suggested activities [43]. Another strength of the current study is that the data are based on semistructured interviews in retrospect three to six years after implementation. Participants were confronted with objective data on the post-implementation results. The results are based on narrative data on the perceptions of professionals, in order to explore how innovations could be sustained in the post-implementation phase. Designs such as longitudinal qualitative research after implementation, or a prospective process log to track and quantify the activities, would probably have been more accurate to identify all the efforts applied in practice during the post-implementation phase. However, merely quantifying the activities performed would not reveal the reasons for perceiving the potential of sustainability activities.

The present study has some limitations. The results may be influenced by the use of a case study design, the sampling of the respondents and hospitals and may be influenced by the analysis. First, a weakness of using a case study design, is the possible bias introduced by the procedures as performed by the researcher [36]. To overcome these, several tactics were performed as described under the heading trustworthiness. A weakness of using a single-case study design is the limited transferability of the results. More case studies will be needed to allow the conclusions to be generalised. The transferability may also be limited as a single-case study design confines the possibility to replicate the results [26]. Second, the results of the current study may be limited by the respondent selection, as most of the respondents had been coordinators or key persons 
during the implementation phase. As a consequence, our data concerning a permanent watch-dog or the involvement of a coordinator may have been relatively underrepresented by the fact that people may not point towards themselves. This might have introduced a selection bias and overly positive answers. Third, the sample only included respondents of hospitals that had achieved successful ERAS implementation at the end of the primary implementation project. It would have enriched the results to include and analyse negative cases of hospitals that might have needed a longer learning curve of implementation. Finally, the use of the classification described by the EPOC group as a theoretical orientation to identify and analyse relevant data needs some further considerations. The results of the present study showed that the EPOC classification can be used to classify sustainability activities. Currently, there is no general taxonomy of implementation strategies. However, the EPOC classification may have led to analysis difficulties in our study, as it may not completely cover sustainability aspects. We have proposed some modifications of the EPOC classification of interventions in relation to sustainability, which may be a starting point for further development of the taxonomy.

The current study showed that sustaining the early post-implementation success of the ERAS programme in colonic surgery needs post-implementation institutional efforts after a hospital has finished a QIC. There is no single strategy to sustain change which is applicable in every context. Given the budget-constrained health care system, it is essential to implement and sustain effective innovations in an efficient way. However, sustainability activities will entail extra costs in addition to the costs of implementation and, where applicable, the costs of the innovation itself. The current study did not yield information on the frequency, intensiveness, exact timing or success of the activities to sustain the implementation successes achieved. More data on the effectiveness and costs of sustainability strategies would be useful for further understanding and prediction of sustainability of innovations.

\section{Conclusions}

This study examined which strategies professionals perceived to be promising for sustaining early post-implementation successes after their hospital joined a QIC. Our findings suggest that joining a QIC may be enough for short-term implementation success but may not be sufficient to achieve long-term normalisation of transformed care. Sustainability of successful early post-implementation results may need a multifaceted bottom-up approach to respond to the complex influences at institutional level. Sustainability planning by hospitals appears to be promising for normalisation of evidence based guidelines and to sustain initially achieved implementation successes. 


\section{CHAPTER 8}

\section{References}

1. Schouten LM, Hulscher ME, van Everdingen JJ, Huijsman R, Grol RP. Evidence for the impact of quality improvement collaboratives: systematic review. Bmj. 2008 Jun 28;336(7659):1491-4.

2. Ayers LR, Beyea SC, Godfrey MM, Harper DC, Nelson EC, Batalden PB. Quality improvement learning collaboratives. Qual Manag Health Care. 2005 Oct-Dec;14(4):234-47.

3. NHS. Sustainability and its relationship with spread and adoption, General improvement skills. Coventry, UK: Improvement Leaders' guide; 2007

4. Gruen RL, Elliott JH, Nolan ML, Lawton PD, Parkhill A, McLaren CJ, et al. Sustainability science: an integrat ed approach for health-programme planning. Lancet. 2008 Nov 1;372(9649):1579-89.

5. Greenhalgh T, Macfarlane F, Barton-Sweeney C, Woodard F. "If we build it, will it stay?" A case study of the sustainability of whole-system change in London. Milbank Q. 2012 Sep;90(3):516-47.

6. Wiltsey Stirman S, Kimberly J, Cook N, Calloway A, Castro F, Charns M. The sustainability of new programs and innovations: a review of the empirical literature and recommendations for future research. Implementation Science. 2012;7(1):17.

7. Scheirer MA. Is Sustainability Possible? A Review and Commentary on Empirical Studies of Program Sustainability. American Journal of Evaluation. 2005 September 2005;26(3):27.

8. Shediac-Rizkallah MC, Bone LR. Planning for the sustainability of community-based health programs: conceptual frameworks and future directions for research, practice and policy. Health Educ Res. 1998 Mar;13(1):87-108

9. Ament SMC, Gillissen F, Maessen JMC, Dirksen CD, van der Weijden T, von Meyenfeldt MF. Sustainability of healthcare innovations (SUSHI): long term effects of two implemented surgical care programmes (protocol). BMC health services research. 2012;12:423.

10. Hoomans T, Ament AJHA, Evers SMAA, Severens JL. Implementing guidelines into clinical practice: what is the value? Journal of Evaluation in Clinical Practice. 2011;17(4):606-14.

11. Doyle C, Howe C, Woodcock T, Myron R, Phekoo K, McNicholas C, et al. Making change last: applying the NHS institute for innovation and improvement sustainability model to healthcare improvement. Implementation Science. 2013;8(1):127.

12. Kehlet $\mathrm{H}$, Mogensen $\mathrm{T}$. Hospital stay of 2 days after open sigmoidectomy with a multimodal rehabilitation programme. Br J Surg. 1999 Feb;86(2):227-30.

13. Basse L, Hjort Jakobsen D, Billesbolle $\mathrm{P}$, Werner $\mathrm{M}$, Kehlet $\mathrm{H}$. A clinical pathway to accelerate recovery after colonic resection. Annals of surgery. $2000 \mathrm{Jul} ; 232(1): 51-7$.

14. Basse L, Raskov HH, Hjort Jakobsen D, Sonne E, Billesbolle P, Hendel HW, et al. Accelerated postoperative recovery programme after colonic resection improves physical performance, pulmonary function and body composition. Br J Surg. 2002;89(4):446-53.

15. Basse L, Thorbol JE, Lossl K, Kehlet H. Colonic surgery with accelerated rehabilitation or conventional care. Dis Colon Rectum. 2004 Mar;47(3):271-7; discussion 7-8

16. Kehlet H, Wilmore DW. Multimodal strategies to improve surgical outcome. Am J Surg. 2002 Jun;183(6):630-41.

17. Varadhan KK, Neal KR, Dejong $\mathrm{CH}$, Fearon KC, Ljungqvist $\mathrm{O}$, Lobo DN. The enhanced recovery after surgery (ERAS) pathway for patients undergoing major elective open colorectal surgery: a meta-analysis of randomized controlled trials. Clin Nutr. 2010 Aug;29(4):434-40.

18. Adamina M, Kehlet H, Tomlinson GA, Senagore AJ, Delaney CP. Enhanced recovery pathways optimize health outcomes and resource utilization: a meta-analysis of randomized controlled trials in colorecta surgery. Surgery. 2011 Jun;149(6):830-40.

19. Sammour T, Zargar-Shoshtari K, Bhat A, Kahokehr A, Hill AG. A programme of Enhanced Recovery After Surgery (ERAS) is a cost-effective intervention in elective colonic surgery. The New Zealand medical jour nal. 2010 Jul 30;123(1319):61-70.

20. Maessen J, Dejong CH, Hausel J, Nygren J, Lassen K, Andersen J, et al. A protocol is not enough to implement an enhanced recovery programme for colorectal resection. Br J Surg. 2007 Feb;94(2):224-31. 
21. Gillissen F, Hoff C, Maessen JMC, Winkens B, Teeuwen JH, von Meyenfeldt MF, et al. Structured synchronous implementation of an enhanced recovery program in elective colonic surgery in 33 hospitals in The Netherlands. World journal of surgery. 2013 May;37(5):1082-93.

22. Coolsen MM, Wong-Lun-Hing EM, van Dam RM, van der Wilt AA, Slim K, Lassen K, et al. A systematic review of outcomes in patients undergoing liver surgery in an enhanced recovery after surgery pathways. HPB : the official journal of the International Hepato Pancreato Biliary Association. 2013 Apr;15(4):245-51.

23. Lv D, Wang X, Shi G. Perioperative enhanced recovery programmes for gynaecological cancer patients. Cochrane Database Syst Rev. 2010 (6):CD008239.

24. Gillissen F, Ament SM, Maessen JMC, Dejong CH, Dirksen CD, van der Weijden T, et al. Sustainability of an Enhanced Recovery After Surgery Program (ERAS) in Colonic Surgery. World journal of surgery. 2014 Aug 23.

25. Yin RK. Case Study Research: Design and Methods: SAGE Publications; 2003.

26. Stake RE. The Art of Case Study Research: SAGE Publications; 1995.

27. Crowe S, Cresswell K, Robertson A, Huby G, Avery A, Sheikh A. The case study approach. BMC medical research methodology. 2011;11(1):100.

28. Tricco AC, Cogo E, Ashoor H, Perrier L, McKibbon KA, Grimshaw JM, et al. Sustainability of knowledge translation interventions in healthcare decision-making: protocol for a scoping review. BMJ open. 2013;3(5). PubMed PMID: 23674448.

29. Grol R, Grimshaw J. From best evidence to best practice: effective implementation of change in patients' care. Lancet. 2003 Oct 11;362(9391):1225-30.

30. Group CEPaOoCR. Data Collection Checklist. Ontario: Institute of Population Health, University of Ottawa, 2011.

31. Hsieh HF, Shannon SE. Three approaches to qualitative content analysis. Qualitative health research. 2005 Nov;15(9):1277-88

32. Ulin PRR, E.T., Tolley, E.E. Qualitative methods in public health, a field guide for applied research. San Francisco: A Wiley Imprint; 2005.

33. van den Hoonaard W. Working with Sensitizing Concepts: Analytical Field Research: Thousand Oaks; 1997.

34. Lincoln YS GE. Naturalistic Inquiry Newbury Park, CA: Sage Publications Inc; 1985.

35. Hulscher ME, Schouten LM, Grol RP, Buchan H. Determinants of success of quality improvement collaboratives: what does the literature show? BMJ Qual Saf. 2013 Jan;22(1):19-31.

36. Knott A, Pathak S, McGrath JS, Kennedy R, Horgan A, Mythen M, et al. Consensus views on implementation and measurement of enhanced recovery after surgery in England: Delphi study. BMJ Open. 2012;2(6)

37. Maessen JMC. Enhanced Recovery After Surgery Fact or Fiction? Maastricht: Maastricht University; 2009.

38. Ovretveit J, Bate P, Cleary P, Cretin S, Gustafson D, Mclnnes K, et al. Quality collaboratives: lessons from research. Quality \& safety in health care. 2002 Dec;11(4):345-51.

39. Pettigrew A, Ferlie E, McKee L. Shaping strategic change - The case of the NHS in the 1980s. Public Money \& Management. 1992 1992/07/01;12(3):27-31.

40. Damschroder L, Aron D, Keith R, Kirsh S, Alexander J, Lowery J. Fostering implementation of health services research findings into practice: a consolidated framework for advancing implementation science. Implementation Science. 2009;4(1):50.

41. Vos L, Duckers ML, Wagner C, van Merode GG. Applying the quality improvement collaborative method to process redesign: a multiple case study. Implementation science : IS. 2010;5:19.

42. ERAS Society [http://www.erassociety.org/]

43. Benzer JK, Beehler S, Cramer IE, Mohr DC, Charns MP, Burgess JF, Jr. Between and within-site variation in qualitative implementation research. Implementation science : IS. 2013;8:4. 

Part 4

Discussion 



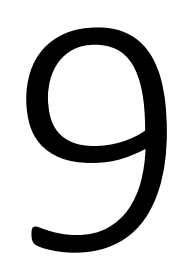

General discussion 
It is well known that substantial gaps may exist between desired care and actual care provided. Frequently, evidence-based innovations are implemented with the aim to increase the quality of care. Unfortunately, the implemented innovations are frequently not maintained causing that many patients do not receive care following the latest evidence. The use of the word 'sustainability' is emerging in the health care field. Also, in relation to implementation of evidence, the concept of sustainability is relatively new and unexplored. After invested implementation efforts it is important that the innovation sustains in practice. If achieved quality improvement results decrease unintendedly in the post-implementation phase, investments and efforts to change practice have been a waste of money and time. Furthermore, teams may get less motivated to be involved in quality improvement projects in case innovations do not sustain. Although the goal of implementation projects is to embed health care innovations in daily routines, many implementation projects focus primarily on immediate results.

The overall objective of this thesis was to improve long-term impact of implementation efforts by exploring the concept of sustainability of health care innovations in hospital care. In this thesis, sustainability was defined as change which exists when a newly implemented innovation continues to deliver the achieved benefits over a longer period of time, certainly does not return to the usual processes and becomes the way things are done around here', even after the implementation project is no longer actively carried out, until a better innovation comes along". Using this definition, we assume that an evidence-based health care innovation is successfully implemented. In our research the level of sustainability was determined in terms of professionals' adherence, patient outcomes and economic value.

The thesis focused on the follow up of quality improvement research of two implementation projects. The Maastricht University Medical Centre (MUMC) was involved in two major implementation projects in the Netherlands between 2006 and 2009: The Netherlands Organisation for Health Research and Development (ZonMW) supported project 'Introduction of a breast cancer care programme in ultra-short stay (ambulatory/24 hour stay setting) (SSP) in four early adopter centres: implementation and evaluation' and the Dutch Institute for Health Care Improvement (CBO) led 'introduction of the enhanced recovery after surgery (ERAS) programme in colonic surgery using a breakthrough implementation technique' $[1,2]$. The implementation projects resulted in remarkable improvements and the aim of this thesis was to analyse to what level both health care innovations (SSP and ERAS) were sustained three to six years after finishing implementation.

This chapter presents an overview of the main findings per research question and places them in the perspective of the existing literature. Also, some methodological issues 
of the thesis are discussed. Finally, the implications of the results for clinical practice and recommendations for future research are described.

\section{Summary and main findings}

The first research question focused on the level of sustainability of health care innovations in the late post-implementation phase. On patient level, care according to SSP and the benefits achieved related to SSP were sustained over a longer period of time. Although slight adaptations in the execution of short stay on hospital level, an aging population and differences in type of surgery were observed; breast cancer surgery care in short stay had become the way things are done in the early adopter hospitals. The results related to the ERAS programme showed that the benefits achieved early after implementation were sustainable, but that the sustainability remains challenging on hospital level following successful implementation. Our findings revealed that the ERAS programme had not fully become the way things are done in the participating hospitals. Taken into account that the case mix had become more complex three to six years following implementation, care according to the ERAS programme seems to have to some degree returned to the traditional processes and was not fully sustained for a longer period of time. Also, in contrast to SSP, the ERAS programme was not spread and embedded in national quality indicators. The actual level of sustainability varied between hospitals and on sustainability indicator level. To compare our results with other studies, a systematic review about the sustainability of professionals' adherence to clinical practice guidelines was conducted. Long-term sustainability of $100 \%$ professionals' adherence compared to the early post-implementation results was revealed in $41 \%$ of the evaluations (7 out of 17). Due to the lack of sustainability research we think that this finding is an overestimation. The studies were heterogeneous with respect to their methodology.

To answer the second research question, which was 'to what level the economic value of SSP was sustained in four early adopter hospitals', the level of sustained economic value of SSP for breast cancer surgery was determined, using the net monetary benefit (NMB) approach. Our results showed that sustainability of economic value of health care innovations can be calculated using the NMB approach. Application of the NMB approach showed that, overall, the economic value sustained five years following implementation of SSP from a societal perspective. The mean pooled iNMB breast cancer surgery care was $€ 1649$ per patient. Bootstrap analysis showed a 95\% probability that the economic value of SSP was sustained irrespective of the threshold value. However, application of the NMB approach for the analysis of sustainability of economic value raised some issues, such as whether regression analysis should be performed to correct for differences in case-mix over time, the appropriate perspective, 
the level of analysis (group or individual hospital level), and the appropriate timing of analysis.

The third research question was aimed on exploring the determinants of the sustainability of the two health care innovations three to six years after implementation, as perceived by the professionals. Modification and adaptability of the programme, costeffectiveness of the innovation, structural characteristics related to the organisation, short communication lines within the multidisciplinary team, culture, benefits to patients, cosmopolitanism between organisations, the existence of external policies and incentives, knowledge and belief in the programme, and spreading the programme to other settings were perceived determinants of sustainability. Largely the same determinants were revealed for the sustainability in both cases. Surprisingly, leadership was not revealed to be a determinant of the sustainability of the SSP and the ERAS programme.

The fourth research question focused on promising strategies for the sustainability of the ERAS programme, as perceived by professionals after the hospitals joined a quality improvement collaborative. Activities targeting professionals and the organisation were perceived to be promising to sustain the ERAS programme in the post-implementation phase. Strategies targeted at professional level were internal audit and feedback on outcomes, small-scale educational booster meetings, and reminders. Strategies that were seen as promising on organisational level were changing the physical structure of the organisation, changing the care process, making work agreements and delegating responsibility and involving a coordinator. In most hospitals such strategies were combined and applied as multifaceted self-driven promising strategy. Taken into account the results related to the determinants of sustainability, the findings suggested that too much focus on systematic and fixed strategies, may limit the flexibility for future quality improvement.

\section{State of the art and what this research adds}

The current thesis contributed to the exploration of the concept of sustainability of health care innovations. The sustainability research presented in this thesis was original with respect to the evaluation of two hospital-based multidisciplinary innovations. To our knowledge, both sustainability evaluation cases were the first studies of surgical care programmes years following finishing implementation. Currently, both innovations are still being implemented in practice $[3,4]$ and up till now no new innovation, which could replace the SSP or the ERAS programme, has come along. We found that in both innovation cases, the level of sustainability differed between hospitals and the sustainability was different on sustainability indicator level. Mixed findings on the level of sus- 
tainability of professionals' adherence were shown by our systematic review confirming earlier findings $[5,6]$.

\section{Sustainability research}

As also highlighted by other authors [6, 7], sustainability research is broadly focused, fragmented, heterogeneous and underdeveloped. This thesis showed that the definition used for sustainability is not mentioned consistently in sustainability evaluations of clinical practice guidelines in medical practice and generally there is no definition of sustainability used. Also, this research confirmed that if a definition for sustainability is used in a sustainability evaluation, they differ over studies [6, 8]. A recently suggested term related to sustainability is sustainment, which is the continued use of an innovation in daily care [9]. Sustainment implies a linear approach to the innovation. However, an innovation may evolve in the post-implementation phase and it may be undesirable to sustain it in the original content or format. By evaluating the level and the determinants of sustainability of two hospital-based multidisciplinary innovations, both a linear and a dynamic approach for sustainability were combined in our research. Combining both approaches gives a more complete picture of the sustainability of the innovations. Not many studies have performed a mixed method approach to study sustainability [6].

\section{Determinants of sustainability}

In the current thesis, the Consolidated Framework for Implementation Research (CFIR) $[10,11]$, was used as a systematic approach gathering and analysing data regarding the determinants of sustainability of the two surgical programmes as perceived by professionals. The five CFIR domains [10], that is, the characteristics of the innovation, individuals involved, inner setting, outer setting and the implementation process, were represented in the results of this thesis. Some determinants beyond the CFIR have emerged in this thesis, i.e. modification of the programme over the years and spread. Probably, the determinants modification and spread were not of relevance during implementation [12]. The professionals involved perceived that the following determinants were essential for the sustainability of SSP and the ERAS programme: the possibility to modify and adapt the programme, cost-effectiveness of the innovation, structural characteristics related to the organisation, short communication lines within the multidisciplinary team, culture, benefits to patients, cosmopolitanism between organisations, the existence of external policies and incentives, knowledge and belief in the programme, and spreading the programme to other settings. Adaptability of the innovation and perceived benefits for the patients were also presented by Scheirer [5], who investigated the determinants of sustainability in the Canadian and U.S. health care setting. Furthermore, in line with the findings of Scheirer, a programme champion, substantial fit with the underlying organisation's mission and procedures, and support from other stakeholders in the community were presented as other determinants of sustainability. Scheirer described a programme champion as 'a person who is strategically located to 
have access to upper management as well as influence on, or control over, day-to-day programme operations'. In contrast with our expectations, leadership was not mentioned as a determinant of sustainability. Respondents in our study perceived that the role of the project leader, in terms of leadership, changed into a more watching role and was delegated to a nurse, with a mandate of the head of the surgery department. In the CFIR, this was coded under 'implementation process' [10]. Also, our study has shown that involvement of a coordinator was perceived as one of the promising activities for the sustainability of the ERAS programme. 'Substantial fit with the underlying organisation's mission and procedures' was revealed for example in terms of planning of care. 'Support from other stakeholders in the community' was perceived by the respondents as a key determinant of sustainability. A determinant that was not shown to be a perceived key determinant of sustainability was the implementation strategy used. Although our aim was not to compare the innovation cases, the initial assumption was that the tailored implementation strategy would increase the chance for the sustainability of the innovation. A tailored implementation strategy is more focused on the needs of the context. A recent study showed that improved quality of care delivery during both the first and second year after programme implementation positively influenced the sustainability of health care programmes [13]. Teams achieving early postimplementation success may have a stronger believe in the innovation. This suggests that the effect of the implementation strategy on early post-implementation achievements may influence the sustainability of the innovation.

\section{Promising strategies}

In public health, sustainability capacity is described as the existence of activities and procedures that enables a public health programme to provide resources to sustain evidence-based policies and activities [8]. Our findings suggest that also in hospital care capacity is a determinant of sustainability as a post-implementation strategy was perceived to be promising to sustain the ERAS programme after the hospital joined a quality improvement collaborative. Some definitions used in implementation research approach sustainability as an ongoing process. For example, Chambers described sustainability as 'the process of managing and supporting the evolution of an intervention within a changing context' [14]. Recent studies revealed activities in terms of evaluation and feedback, training and education [15], and integration of rules [6]. Ploeg et. al revealed four types of sustainability strategies, in the context of innovation in home care for older adults, namely evaluation and feedback, integrating into practice, educating and coaching, and engaging and communicating [16]. These activities show a notable overlap with the activities used as part of the hospital tailored implementation strategy to implement SSP. They also suggested that executing sustainability strategies is a continuous process facilitating the spread of the innovation to other settings as it may make the benefits more transparent. This could be confirmed by our findings as the short stay programme was successfully implemented alongside a research project. After successful 
implementation the National Breast Cancer Audit (NABON) and the Health Care Inspectorate (IGZ) adopted some recommendations for short stay in their quality indicators. Transparency of the results in terms of scientific publications and the performance as monitored and revealed by national registries may have had a role in the spread of the clinical practice guideline. In contrast, the normalisation of the ERAS programme into external policies was not observed or mentioned by respondents.

\section{Methodological considerations}

\section{Study design and case selection}

The main part of the thesis covers the long-term follow-up of two innovations three to six years following implementation. A longitudinal multiple-case study approach was applied, as it gave the possibility to track and study in-depth; two innovation cases before, immediately after and late after successful implementation. Case studies are a preferred strategy when questions of 'how' or 'why' are being posed, when the investigator has little control over events and when the focus is on a contemporary phenomenon within a complex real-life context [17]. However, as the selected cases in this thesis are hospital-based, generalisability of the findings to other health care sectors such as primary care or public health may be limited. Furthermore, a sustainability evaluation of another type of innovation, such as an innovation containing a single component or a easy to use medicine, may lead to other results. On the other hand, using a multiplecase study design, although both in the surgery setting, may positively impact on the generalisability of the results [17]. Moreover, medical care is increasingly provided in a complex and multidisciplinary context. The nature of the two innovations studied in this thesis is highly complex and multidisciplinary and the results may increase the generalisability for comparable contexts.

In theory, mixed method research is described as 'the class of research where the researcher mixes or combines quantitative and qualitative research techniques, methods, approaches, concepts or language into a single study' [18]. In this research, a mixedmethod sequential follow-up explanatory design was used in which quantitative research is followed by qualitative research $[19,20]$. First, sustainability of the two innovations was studied quantitatively to analyse the level of sustainability of the innovation. Second, a qualitative method was applied, using the quantitative data during the interviews, to explore the sustainability of the two surgical innovations and to explain the quantitative results. A strength of using this design is that the professionals' view with respect to the actual performance achieved can be studied in-depth. This design is especially useful when unexpected results arise from a quantitative study [21] and in our study the level of sustainability of the innovations was unknown before the start of our study. The use of a mixed method approach is also in line with the sustainability 
research recommendation made by Greenhalgh, as she suggested that combining both study approaches produces an enriched insight in sustainability [22].

The short-term effectiveness of the implementation strategies was not evaluated by robust experimental designs during the primary implementation studies [1, 2], but by pre-post intervention study designs. If the original studies would have been RCTs on the level of the hospitals it might have been interesting to follow-up the original control conditions to monitor the natural course of, for example, the SSP. Our follow-up study was by nature uncontrolled, as there was no intervention to be tested or introduced in the post-implementation phase. Also, the aim of this research project was not to investigate the causal effects of the implementation strategies. Therefore, the uncontrolled design for the exploration of sustainability was in this research project the optimal design.

\section{Hospital selection}

Hospitals with early post-implementation success were selected for participation in the SUSHI-study. In the SSP case the four early adopter hospitals were included, that were shown successful in reaching the implementation targets. The hospitals were labelled as early adopter hospitals with respect to breast cancer surgery in the Netherlands. In the ERAS case, ten out of 33 hospitals were selected. Preferably, all 33 hospitals that were originally exposed to the Breakthrough strategy to implement the ERAS programme should have been included to assess long-term sustainability of the innovation, as it may be possible that hospitals without early implementation success, achieved higher levels of uptake of the ERAS programme in the post-implementation phase due to a slow learning curve. This would imply, as for example was suggested in the SSP case, that the implementation effect in these hospitals may have been measured too soon after implementation. A recent study demonstrated that improved quality of care delivery during both the first and second year after programme implementation positively influenced the sustainability of these programmes [13]. Teams that were successful in implementing a programme were suggested to be more likely to maintain the innovation. Therefore, the hospital selection used in this explorative research project may be a strength, as the chance of finding determinants of sustainability may be higher in hospitals that achieved early post-implementation success.

\section{Measurements}

The measurements early after implementation were conducted prospectively, while the late post-implementation measurements were performed retrospectively. An exception is the cost and QALY measurement for calculation of the level of sustainability of economic value, which required a prospective approach. The difference in prospective and retrospective measurement may have influenced the results. Prospective measurements may influence professionals' behaviour and may consequently result in an overestimation of results. To overcome this possible effect, the retrospective audit with 
respect to patient outcomes and professionals' adherence was performed over the period before the SUSHI study started.

The same measurements as in the primary implementation studies were used in the SUSHI study. To perform long-term evaluations, we were dependent on the availability of data from the primary implementation studies. With respect to the ERAS programme, the cost-effectiveness and the sustainability of economic value of the innovation could unfortunately not be analysed as part of the SUSHI study, due to a lack of data from the primary implementation study. Therefore, costs of the ERAS programme for colonic surgery in the ten participating hospitals was not determined three to six years following implementation.

As mentioned before, not much is known about the appropriate timing of an analysis of sustainability. We used an evaluation time frame of three to six years after finishing implementation. However, such a long-term evaluation could be disturbed by for example updates of the clinical practice guideline, as most handbooks recommend to update clinical practice guidelines two to three years following implementation and $23 \%$ of the handbooks recommend a time frame of four to five years [23]. Also, in the Netherlands the average hospital length of stay for all causes decreased from 8.5 days in 2000 to 5.6 days in 2009 [24]. More care is also delivered in outpatient settings instead of inpatient settings [25]. Hence, using a time frame of three to six years following implementation more developments related to sustainability may have taken place and more determinants of sustainability may have been revealed than if a shorter timeframe had been used. Also, our research suggested that developments in context of the innovation may be a characteristic of sustainability and as such acceptable. Interestingly, we refer in this sustainability research to SSP and the ERAS programme as innovations. However, in the settings we studied the programmes were not perceived as innovations anymore, but as 'care as usual'.

\section{Implications for practice}

The results of this thesis may help and inspire people involved in quality improvement projects, researchers, implementation experts, policy makers, insurance companies and other people working or interested in the complex health care field. Integration of the main findings in future quality improvement projects could facilitate the chance for sustainability of evidence-based innovations in daily clinical practice and ultimately adds to the sustainability of the health care system from a macro-system perspective.

Sustainability of an innovation is difficult to determine and to plan in the preimplementation phase. During the implementation strategy, sustainability of the inno- 
vation is suggested to be discussed and a strategy for sustainability needs to be set when approaching the end of the implementation projects as the determinants of successful primary implementation may be different in comparison with the determinants of sustainability. The results suggest that sustainability planning is an on-going exercise and it is recommended to integrate the sustainability planning and control in quality assurance policies at a hospital level. Furthermore, as also shown in this thesis, isolated hospital practice is not recommended. In case of multi-centre implementation, sharing experiences and site visits may inspire and excel performance in the postimplementation phase. New insights and updates of the innovation may become available, and not integrating new findings in clinical practice guidelines may impact the level of sustainability of the guideline.

Currently, teams may trust the implementation strategy and may perceive that for example joining a guided implementation strategy of a evidence-based innovation is enough for sustained practice change. However, the results showed that, if early postimplementation success was achieved, sustainability was not self-evident on institutional and on sustainability indicator level. Furthermore, professionals may have different (mostly overestimated) perceptions about the actual health care performance. To determine influences on sustainability, professionals were asked to reflect on their team's performance three to six years following implementation. Merely, professionals' perceptions about their performance did not fully reflect the real world performance as they thought that the performance was better. Chapter 9 showed that a multifaceted sustainability strategy was applied or was suggested in all hospitals which achieved successful implementation of the ERAS programme early after joining a quality improvement collaborative. However, the content of the activities must correspond to the needs of the hospital, team and to the needs of the innovation. For example, the sustainability planning for the uptake of a single and easy to use drug may be different than for a multidisciplinary complex clinical practice guideline. Clearly, sustainability evaluation is not without efforts and costs. In a time when the health care budget is under pressure and health care needs to be administered for different internal and external purposes, additional administrative efforts to facilitate sustainability of evidence-based innovations may be perceived as undesired. The value of sustainability must be kept in mind, meaning that a balance must be sought in investments and benefits of sustainability activities. Optimal resource use and effective care delivery is needed to keep health care at an accessible level. Possibly, health care providers and hospitals may jointly contribute to the resources for sustainability in terms of educational booster sessions or by audit and feedback by means of using data from national registries. Input of national registries may partly be automatised using hospital data. 
Implications for the two innovation cases

The sustainability of two innovations, SSP and the ERAS programme, were studied in depth by means of various evaluations. The findings revealed in this thesis may have practice implications related to both cases.

\section{Case I: Short Stay Programme for breast cancer surgery}

\section{Close the gap}

In chapter 3, some variation in the level of sustainability in terms of proportions of patients treated in short stay was observed between the early adopter hospitals. However, the current thesis showed that the differences in proportion of patients treated in short stay between early adopter hospitals became smaller. The national mean hospital length of stay for breast cancer surgery was 1.5 (sd: 1.5) in 2011 (Netherlands Cancer Registry) compared to 0.9 day (sd: 0.8) found in our study. Based on the findings in our study it could be concluded that care may be delivered more efficiently in hospitals where length of hospital stay after breast cancer surgery is relatively high. However, long-term experience with the SSP showed that hospital length of stay was affected by type of surgery in the early adopter hospitals. One hospital in the breast cancer case was shown to performed significantly more immediate breast reconstructions. In the Netherlands, not all hospitals perform immediate reconstructions.

Breast cancer is the cancer type with the most scientific biomedical publications [26]. Continuously, breast cancer care is evolving and is becoming more specialised. The audit showed that the adherence to recommendation 'breast nurse stays in contact with the patient after short stay (phone consultation)' was low five years following implementation. Chapter 7 confirmed that this recommendation was not needed for most patients as the patients are well informed and the care process is well organised. Some new recommendations may have emerged as a result of the increased treatment of immediate breast reconstructions. Also, patients were significantly older five years following implementation. Furthermore, the long-term experience and the trust in SSP may influence the content of new recommendations for treating breast cancer patients in short stay. An update of the key recommendations for breast cancer surgery in short stay is recommended and should be disseminated to hospitals performing breast cancer surgery to decrease the gap between theoretical care and practice, This may lead to more efficient care delivery on national level.

\section{Sustained partnership}

Treating (breast cancer) patients in short stay not only depends on structured hospital care but also on well organised and embedded integrated care delivery. Discharged patients must be able to contact specialised care at any moment after discharge. Therefore, sustained partnership with extramural professionals is needed [22, 27]. Due to the 
long-term collaboration with specialised home care givers, respondents of the SSP case indicated that they could rely on this partnership. Unfortunately, as a result of national budget cuts and decentralisation of responsibility to communities, differences between communities regarding the availability and quality of home care may become larger. Changes in home care availability may influence the sustainability of breast cancer surgery treatment in short stay as it could impact on the post-discharge procedures and patient satisfaction. It could even influence the hospital length of stay. Also, losing the trusted partnership between the hospital and external stakeholders could lead to confusion and inefficiencies within the care system.

\section{Specialisation of care}

Increasingly, hospitals need to make strategic choices regarding the focus of care delivery. Specialisation and concentration of complex treatments [28], in this case breast cancer surgery, may lead to increased quality of care [29]. However, treating more breast cancer patients per hospital may lead to decreased dedicated time by the breast cancer nurse, who serves as a valuable and reliable case manager for patients. As this research, and the research related to the original implementation study, showed, patient information is essential for treating breast cancer surgery patients in short stay. Respondents mentioned that increased patient volumes may lead to less time for patients and limited overview of the department. As a consequence, the sustainability of treating breast cancer patients in short stay may decrease.

\section{Case II: Enhanced Recovery After Surgery for colonic surgery}

\section{Long-term and wide experience}

The applicability of the ERAS programme is expanding and a recent systematic review showed that the programme is even safe for patients of 65 years and older [30]. Also, research showed that patients' and health care professionals' attitudes for the ERAS programme were mainly positive, and this confirms the trust and acceptance of the innovation [31]. According to the qualitative results presented in this thesis, trust in the innovation facilitates its sustainability, Moreover, the ERAS programme in colonic surgery has been implemented widely, and also in other specialties the ERAS programme has been analysed and adopted [32-34]. However, our research showed that the programme was not fully sustained in all participating hospitals achieving early postimplementation success. This result suggests normalisation of deviance and calls for additional focus on the programme after finishing the implementation strategy on institutional level.

\section{Attention for adherence to post-operative ERAS programme elements}

It was demonstrated that the ERAS programme was not sustained in all hospitals with primary implementation success. A wide hospital variation in performance according to 
the ERAS programme was observed. The sustainability of the adherence to the postoperative ERAS programme was perceived to be more difficult to achieve than sustainability of preoperative and operative care elements. Especially the adherence to postoperative programme elements as 'mobilisation of patients for more than three hours on the first postoperative day,' 'cessation of intravenous fluids on the first postoperative day' and 'resumption of solid foods decreased. These ERAS programme elements, especially carried out by the nursing staff, may need extra attention during and after implementation. Also, practice variation with respect to hospital length of stay was observed and our research suggested that the management of hospital length of stay could be improved. Stakeholders, involved in future quality improvement projects and research related to the ERAS programme, may include these lessons in their context.

\section{ERAS and discharge}

The results of our research revealed that SSP was better sustained than the ERAS programme in terms of hospital length of stay. Moreover, our qualitative findings showed that the possibility for modification of the guideline was a determinant of sustainability. The version of the ERAS programme contains merely care-related items and there may be room for an update of the innovation.

Both our quantitative and qualitative research results imply that there is room for an update of the innovation. Thus, the sustainability of the ERAS programme with respect to hospital length of stay may be facilitated by adopting organisational elements in the guideline, such as multidisciplinary surgical team meetings to discuss the surgical treatment at patient level. Structural meetings of professionals from all disciplines involved, varying from surgeon to dietist facilitates the communication between disciplines and structures the policy and treatment. Also, after agreements concerning treatment, the patient will receive the same message from all parties during the care process. Clear and unambiguous information about the treatment policy to patients will probably strengthen the patients' adherence to ERAS programme elements and will probably empower them in discharge decision-making.

Discharge criteria were suggsted by the ERAS Society but they are not part of the guideline as the guideline aims to facilitate fast recovery instead of early discharge. Currently, discharge criteria seem to be well-known by the professional but apperently these criteria are not applied in every team. This may suggest a difference in mindset with respect to discharge between the ERAS programme case and the SSP case. The criteria may be adopted in the ERAS programme in order to let patients go home at the moment admission is no longer needed and potentially harmful. 


\section{Communication between hospitals}

In the ERAS case, the communication between hospitals with respect to performance may be improved to facilitate the sustainability of the innovation. The ERAS society was founded in 2010 and has started to be an international leader in the field of the ERAS programme (www.erassociety.org). One of the aims of the ERAS Society is to continuously review and update the literature and to facilitate the implementation of the ERAS programme. Another facilitating activity of the ERAS Society is the ERAS Congress, which has been organised in 2012, 2014 and in 2015. Although the ERAS society undoubtedly facilitates communication between hospitals about the ERAS programme and its sustainability in practice, the respondents in our study perceived still not enough communication between hospitals in the Netherlands with respect to the ERAS programme.

\section{External policies}

The results presented in this thesis revealed that there is a gap between actual care and desired care according to the ERAS programme and that there is a gap between functional recovery and hospital length of stay. Moreover, our qualitative research suggested that external policies may influence the sustainability of health care innovations. Therefore, it is recommended to insert ERAS programme elements in the Dutch Institute for Clinical Auditing (DICA) system. Furthermore, insurance companies may facilitate the sustainability of the ERAS programme in terms of recognising hospital length of stay related to and defined by discharge indicators. These discharge indicators must be described and in case patients undergo unnecessary hospital admission days; the redundant and potentially harmful part of care may not be reimbursed by the insurance company.

\section{Implications for future research}

\section{Value of the ERAS programme}

The sustainability of economic value of the ERAS programme was not analysed in this thesis. Three recent systematic reviews showed that very few studies focused on the cost-effectiveness of the ERAS programme [35-37]. All reviews concluded that the evidence is methodologically weak they tend to support the cost-effectiveness of the ERAS programme compared to traditional care. More well-designed research including costs outside the health care perspective is needed to analyse the cost-effectiveness of the ERAS programme. The current research presented the findings of the level of sustainability of the ERAS programme in terms of patient outcomes and adherence to elements of the ERAS programme. Sustainability of innovations in terms of, for example, health outcomes and adherence to programme elements, does not automatically imply sustainability of economic value. It would be interesting to analyse what the value of the ERAS programme is. 


\section{Sustainability research}

The current thesis confirmed that sustainability research is heterogeneous and underdeveloped. For example, there is no commonly accepted timeframe, standard definition or method to assess the sustainability of quality improvements. Chapter 6 showed that few studies analysed the sustainability of professionals' adherence to a clinical practice guideline. The methods used in these studies were variable and conclusions concerning sustainability were mainly based on reflections of the author.

The concept of sustainability in the current thesis was studied from a multiple dimensional approach and indicated additional key points for future research. In our research the level of sustainability was determined in terms of professionals' adherence, patient outcomes and economic value. Possibly, other sustainability indicators may be explored such as the level of sustained knowledge (38) of an innovation. However, evaluating the sustainability of innovations is time consuming and therefore the development of more simple or efficient sustainability evaluations need to be explored. Also, sustainability research needs to focus on what strategies are superior in what case, rather than assessing their value only as 'promising'. Strategies to establish sustainability may not always be necessary as new routines may be sustained without additional efforts.

The analyses of sustainability of the innovations raised some issues, such as whether regression analysis should be performed to correct for differences in case-mix over time, the appropriate perspective of economic evaluation, the level of analysis (macro or meso level), and the appropriate timing of analysis. This research showed for example that an extended learning process of SSP in the post-implementation phase resulted in increased economic value. Respondents perceived 'trust and believe in the innovation' as an important determinant of the sustainability while long-term experience was indicated to strengthen the trust and believe in SSP. Long-term experience with SSP may have influenced the application of the programme to older patients and more complex patient groups. It is therefore questionable if regression analyses are needed to adjust for changes in case-mix over time, as it in itself may be a characteristic of sustainability. Moreover, the role of being an early adopter, leadership and the use of a tailored implementation strategy in relation to sustainability must be analysed. More sustainability research is needed to answer these conceptual and methodological questions. Also, more case study research must be performed in various settings, in order to provide more robust findings. Based on our results, it is recommended to develop a general framework for the evaluation of sustainability of health care innovations.

\section{The patient}

Health care is a dynamic and complex field and ultimately health care is delivered around patients' needs. The last decade, the role of the patient and patient's needs have been changing. Increasingly, the patient has become more articulated and em- 


\section{CHAPTER 9}

powered by more information. In our research, patient information was an important element of both innovations. It would be interesting to know what the role of patient's awareness and knowledge of the innovation is in relation to the sustainability of a health care innovation. Empowering the patient with patient versions of a clinical practice guideline or a decision aid may influence the sustainability of the innovation. Improved communication about the admission until discharge (including a decision aid with the discharge criteria) may increase the chance for sustainability of the benefits related to the ERAS programme. Moreover, it would be interesting to know how patients experience the clinical practice guidelines. Gaps in actual care and desired care as proposed by patients could lead to appropriate adaptations and may further increase the quality of the guidelines and consequently, the sustainability of the innovations. In the current study we measured quality of life as perceived by the patient. However, it would be interesting to know whether or not the quality of care as perceived by the patient was sustained three to six years after implementation. To our knowledge, no sustainability evaluation has included this type of data to determine the role of the patients regarding the sustainability of an innovation.

\section{Conclusions}

The gap between actual health care and desired practice is substantial and as a result many patients receive suboptimal and potential harmful care. Also, as the demand for health care increases and the resource availability is limited, focus on quality improvement is needed. To guarantee quality care, it is important that cost-effective innovations sustain after implementation in practice and that traditional routines hindering the execution of such innovations become eliminated. The thesis revealed gaps in current sustainability research and the insights of this thesis may be a starting point for further sustainability research and projects. Hopefully, this thesis created awareness about the need for sustainability of evidence-based health care innovations in the realworld to safeguard the time and money invested to improve medical care. 


\section{References}

1. Maessen JMC. Enhanced Recovery After Surgery Fact or Fiction? Maastricht: Maastricht University; 2009.

2. De Kok M. Costs and effects of implementation of a short admission programme following breast cancer surgery in the Netherlands. Maastricht; 2010.

3. Geltzeiler CB, Rotramel A, Wilson C, Deng L, Whiteford MH, Frankhouse J. Prospective study of colorectal enhanced recovery after surgery in a community hospital. JAMA surgery. 2014 Sep;149(9):955-61.

4. Hainsworth AJ, Lobo CR, Williams P, Case C, Surridge F, Sharma AK, et al. '23 h Model' for breast surgery: an early experience. Breast (Edinburgh, Scotland). 2013 Oct;22(5):898-901.

5. Scheirer MA. Is Sustainability Possible? A Review and Commentary on Empirical Studies of Program Sustainability. American Journal of Evaluation. 2005 September 2005;26(3):27.

6. Wiltsey Stirman S, Kimberly J, Cook N, Calloway A, Castro F, Charns M. The sustainability of new programs and innovations: a review of the empirical literature and recommendations for future research. Implementation science : IS. 2012;7:17.

7. Gruen RL, Elliott JH, Nolan ML, Lawton PD, Parkhill A, McLaren CJ, et al. Sustainability science: an integrated approach for health-programme planning. The Lancet. 2008;372(9649):1579-89.

8. Schell S, Luke D, Schooley M, Elliott M, Herbers S, Mueller N, et al. Public health program capacity for sustainability: a new framework. Implementation Science. 2013;8(1):15.

9. Aarons GA, Hurlburt M, Horwitz SM. Advancing a conceptual model of evidence-based practice implementation in public service sectors. Administration and policy in mental health. 2011 Jan;38(1):4-23.

10. Damschroder LJ, Aron DC, Keith RE, Kirsh SR, Alexander JA, Lowery JC. Fostering implementation of health services research findings into practice: a consolidated framework for advancing implementation science. Implementation science : IS. 2009;4:50.

11. Damschroder $\sqcup$, Hagedorn HJ. A guiding framework and approach for implementation research in substance use disorders treatment. Psychol Addict Behav. Jun;25(2):194-205.

12. Grol R, Grimshaw J. From best evidence to best practice: effective implementation of change in patients' care. Lancet. 2003 Oct 11;362(9391):1225-30.

13. Cramm JM, Nieboer AP. Short and long term improvements in quality of chronic care delivery predict program sustainability. Social Science \& Medicine. 2014 1//;101(0):148-54.

14. Chambers D, Glasgow R, Stange K. The dynamic sustainability framework: addressing the paradox of sustainment amid ongoing change. Implementation Science. 2013;8(1):117

15. Doyle C, Howe C, Woodcock T, Myron R, Phekoo K, McNicholas C, et al. Making change last: applying the NHS institute for innovation and improvement sustainability model to healthcare improvement. Implementation Science. 2013;8(1):127.

16. Ploeg J, Markle-Reid M, Davies B, Higuchi K, Gifford W, Bajnok I, et al. Spreading and sustaining best practices for home care of older adults: a grounded theory study. Implementation Science. 2014;9(1):162.

17. Yin RK. Case Study Research: Design and Methods: SAGE Publications; 2003.

18. Johnson RB, Onwuegbuzie AJ. Mixed Methods Research: A Research Paradigm Whose Time Has Come. Educational Researcher. 2004 October 1, 2004;33(7):14-26.

19. Ivankova NV, Creswell JW, Stick SL. Using Mixed-Methods Sequential Explanatory Design: From Theory to Practice. Field Methods. 2006 February 1, 2006;18(1):3-20.

20. Creswell JW, Gutmann ML, Hanson WE. Advanced mixed methods research designs. Handbook of mixed methods in social and behavioral research. 2003:209-40.

21. Morse JM. Approaches to qualitative-quantitative methodological triangulation. Nursing research. 1991 Mar-Apr;40(2):120-3.

22. Greenhalgh T, Macfarlane F, Barton-Sweeney C, Woodard F. "If we build it, will it stay?" A case study of the sustainability of whole-system change in London. Milbank Q. 2012 Sep;90(3):516-47.

23. Vernooij R, Sanabria A, Sola I, Alonso-Coello P, Martinez Garcia L. Guidance for updating clinical practice guidelines: a systematic review of methodological handbooks. Implementation Science. 2014;9(1):3.

24. OECD. Average length of stay in hospitals", in Health at a Glance 2011: OECD Indicators. 2011

25. Krystyna-Wasowicz D. Trends in day surgery in the Netherlands. Enschede: Universiteit Utrecht; 2008. 


\section{CHAPTER 9}

26. Bryant J, Boyes A, Jones K, Sanson-Fisher R, Carey M, Fry R. Examining and addressing evidence-practice gaps in cancer care: a systematic review. Implementation Science. 2014;9(1):37.

27. Scheirer MA, Dearing JW. An agenda for research on the sustainability of public health programs. American journal of public health. 2011 Nov;101(11):2059-67.

28. Ziekenhuizen NVv. Zorg in de toekomst. NVZ Standpunten. Utrecht: NVZ, 2013.

29. Gooiker GA, van Gijn W, Post PN, van de Velde CJ, Tollenaar RA, Wouters MW. A systematic review and meta-analysis of the volume-outcome relationship in the surgical treatment of breast cancer. Are breast cancer patients better of with a high volume provider? European journal of surgical oncology : the journa of the European Society of Surgical Oncology and the British Association of Surgical Oncology. 2010 Sep;36 Suppl 1:S27-35.

30. Bagnall NM, Malietzis G, Kennedy RH, Athanasiou T, Faiz O, Darzi A. A systematic review of enhanced recovery care after colorectal surgery in elderly patients. Colorectal disease : the official journal of the Association of Coloproctology of Great Britain and Ireland. 2014 Jul 18.

31. Hughes M, Coolsen MM, Aahlin EK, Harrison EM, McNally SJ, Dejong CH, et al. Attitudes of patients and care providers to enhanced recovery after surgery programs after major abdominal surgery. The Journal of surgical research. 2014 Jun 23.

32. Coolsen MM, Wong-Lun-Hing EM, van Dam RM, van der Wilt AA, Slim K, Lassen K, et al. A systematic review of outcomes in patients undergoing liver surgery in an enhanced recovery after surgery pathways. HPB : the official journal of the International Hepato Pancreato Biliary Association. 2013 Apr;15(4):245-51.

33. Findlay JM, Gillies RS, Millo J, Sgromo B, Marshall RE, Maynard ND. Enhanced recovery for esophagectomy: a systematic review and evidence-based guidelines. Annals of surgery. 2014 Mar;259(3):413-31.

34. Ibrahim MS, Alazzawi S, Nizam I, Haddad FS. An evidence-based review of enhanced recovery interventions in knee replacement surgery. Annals of the Royal College of Surgeons of England. 2013 Sep;95(6):386-9.

35. Adamina M, Kehlet H, Tomlinson GA, Senagore AJ, Delaney CP. Enhanced recovery pathways optimize health outcomes and resource utilization: a meta-analysis of randomized controlled trials in colorectal surgery. Surgery. 2011 Jun;149(6):830-40.

36. Lee L, Li C, Landry T, Latimer E, Carli F, Fried GM, et al. A systematic review of economic evaluations of enhanced recovery pathways for colorectal surgery. Annals of surgery. 2014 Apr;259(4):670-6.

37. Paton F, Chambers D, Wilson P, Eastwood A, Craig D, Fox D, et al. Effectiveness and implementation of enhanced recovery after surgery programmes: a rapid evidence synthesis. BMJ Open. 2014 July 1 , $2014 ; 4(7)$

38. Edmunds J, Read K, Ringle V, Brodman D, Kendall P, Beidas R. Sustaining clinician penetration, attitudes and knowledge in cognitive-behavioral therapy for youth anxiety. Implementation Science. 2014;9(1):89. 
Summary 
This thesis focused on the measurement and concept of sustainability of health care innovations that have been actively implemented in hospital care. Many teams that are involved in changing and improving patient care focus on the primary implementation phase. However, it is often unknown to what level the quality improvements have been sustained. Sustainability of short-term implementation successes is a relatively new concept and various definitions and perspectives are used in health care research. Furthermore, not much research has examined the level and the determinants of sustainability of successfully implemented innovations.

The overall objective of this thesis was to explore the concept of sustainability of health care innovations that have been actively implemented. Two innovations, the enhanced recovery after surgery (ERAS) programme for colonic surgery and a short stay programme for breast cancer surgery (SSP), were studied by means of various types of evaluations three to six years following implementation. The ERAS programme was implemented between 2006 and 2009 using a breakthrough implementation strategy in 33 hospitals and SSP was implemented between 2006 and 2007 using a hospital tailored implementation strategy in four early adopter hospitals.

\section{Part I Introduction}

The first part (chapters $1-2$ ) of the thesis, reports on the background of the research. This part contains the introductory chapter, chapter 1 , and the study protocol of the 'sustainability of health care innovations' (SUSHI) study, chapter 2.

Chapter 1, the general introduction, explains the relevance of the subject and an overview of the most important elements of the study. Sustainability and concepts related to sustainability such as innovation, quality improvement and implementation are outlined. Furthermore, the background of the two innovation cases is presented. Finally, the aim of the thesis is described and the following research questions of the thesis are presented:

- To what level were health care innovations sustained in the late post-implementation phase?

- To what level was the economic value of the short stay programme for breast cancer surgery sustained in four early adopter hospitals?

- What were the determinants of the sustainability of the two innovations three to six years after implementation, as perceived by the professionals?

- What strategies seem to be promising for sustaining the ERAS programme for colonic surgery, as perceived by the professionals?

Chapter 2 presents the methodology of the SUSHI study. A mixed methods approach was used to explore the concept of sustainability, by analysing two innovations three to six years following successful implementation. The measured benefits were patient outcomes such as functional recovery and length of hospital stay and the pro- 
fessionals' adherence to the programmes. Additionally, the economic value was recalculated for SSP from a societal perspective. The results achieved three to six years following implementation were compared with the (economic) results immediate after implementation. Key persons involved in the care process were interviewed to explore the determinants of sustainability of the two innovations.

\section{Part II Quantitative analyses of the level of sustainability}

In the second part (chapters $3-6$ ), the level of sustainability of SSP and the ERAS programme is evaluated from a quantitative research approach. Sustainability is evaluated in terms of patient outcomes, professionals' adherence and economic value. The level of sustainability in terms of patient outcomes and the professionals' adherence to SSP and the ERAS programme elements is presented in chapters 3 and 4 respectively. In chapter 5 , the analysis of the sustainability of economic value is presented using SSP as an exemplary case. In chapter 6 , a systematic review of the sustainability of professionals' adherence to clinical practice guidelines in medical care is presented.

Chapter 3 investigates the sustainability of SSP five years following implementation. A retrospective audit of 160 consecutive patients undergoing breast cancer surgery was performed in the four early adopter hospitals. Sustainability of the primary implementation success was assessed by measuring the proportion of patients treated in short stay (day or 24 hour admission) and professionals' adherence to the key recommendations to facilitate SSP. Data of the retrospective audit were analysed on patient and on hospital level and were compared with the data of the primary implementation study $(n=163)$. The overall proportion of patients treated in short stay was $82 \%$ (hospital 1 : 83\%, hospital 2: 78\%, hospital 3: 87\%, hospital 4: 80\%) five years after implementation, which was comparable to the proportion in short stay immediately after implementation ( $p=0.938$ ). Overall professionals' adherence to the SSP key recommendations increased from $65 \%$ directly after implementation to $78 \%$ five years after implementation. This study shows that short stay for breast cancer surgery was overall successfully sustained in the four early-adopter hospitals five years following implementation and that breast conserving surgery was a predictive factor for successful short stay treatment (OR 95\% Cl 4.3 1.6-11.2).

Chapter 4 focuses on the sustainability of the ERAS programme for colonic surgery three to six years after finishing a breakthrough implementation strategy. From the 33 ERAS hospitals in the primary implementation study, ten hospitals that had achieved early post-implementation results were selected. Success was defined as a median hospital length of stay of six days or lower and a professionals' adherence rate above $70 \%$. In 2012, a retrospective audit of 30 consecutive patients was performed in each of these hospitals. Data were compared with the early post-implementation data $(n=532)$. Overall median LOS in the selected hospitals increased from 5.25 days to 6 days, but 
this change was not significant. Time needed for functional recovery was equal in both measurements: median 3.00 days (IQR P25 2.88 - P75 3.00; min. 2.00 - max. 4.00) late post-implementation data vs. median functional recovery 3.00 (IQR P25 3.00 - P75 3.50; min. 3.00 - max. 4.00) early post-implementation data ( $P=0.26)$. Protocol adherence decreased from 75\% (SD $7.6 \mathrm{~min} .64 \%$ - max. 87\%) to 67\% (SD $6.3 \mathrm{~min} .56 \%$ - $\max .73 \%$ ) $(p=0.052)$. Especially adherence to post-operative care elements decreased considerably. This chapter shows that the ERAS programme in colonic surgery was sustained reasonably in the ten selected hospitals. There was quite some variation between the hospitals and on sustainability indicator level.

Chapter 5 introduces the sustainability of economic value using the net monetary benefit approach. Economic value of SSP was determined at two moments in time: a measurement in the early post-implementation phase (POST, i.e. directly following implementation, in 2007, $\mathrm{n}=127$ ) and a measurement in the late post-implementation phase (LATE POST, i.e. five years following implementation, in 2012, $n=121$ ). Using the net monetary benefit (NMB) approach with a maximum willingness to pay for a QALY of $€ 80.000$, the economic value of SSP was fully sustained with a mean pooled iNMB LATE $_{\text {. }}$ POST of $€ 1649$ per patient. The iNMB decreased slightly in the LATE POST group compared to the POST group after adjustment for age category. From a health care perspective, the $\mathrm{NNMB}_{\text {LATE POST }}$ was $€ 487$. The level of sustainability of economic value varied between hospitals, both from a societal and from a health care perspective. Bootstrap analyses showed that the probability that the economic value of SSP was sustained was $95 \%$ and $65 \%$ respectively from a societal perspective and a health care perspective. Application of the NMB approach for the analysis of sustainability of economic value raised some issues, such as whether regression analysis should be performed to correct for differences in case-mix over time, the appropriate perspective, the level of analysis (macro or meso level), and the appropriate timing of the analysis.

Chapter 6 presents a systematic review which is focused on the sustainability of professionals' adherence to clinical practice guidelines in medical practice more than one year following the end of active implementation. Thirteen studies met the inclusion criteria, involving seventeen sustainability evaluations. The mean timeframe between the end of active implementation and the sustainability evaluation was 2.7 years [min 1.5 - max 7.0]. Long-term sustainability of professionals' adherence was revealed in $41 \%$ of the evaluations (7 out of 17 ), adherence was not sustained in five evaluations, four evaluations showed mixed sustainability results and in one evaluation it was unclear whether the professional adherence was sustained. Professionals' adherence was sustained at least at a level of $90 \%$, in $53 \%$ (9 out of 17) of the evaluations. The results of this review suggest that updates of the clinical practice guidelines may have led to a warranted decrease in the adherence to the original clinical practice guideline. Due to the limited number of studies, the absence of a uniform definition, the high risk of bias, and the mixed results of studies, no firm conclusion about the sustainability of professionals' adherence to guideline recommendations in medical practice can be drawn. 


\section{Part III Qualitative analyses of sustainability}

Part three (chapters $7-8$ ) focuses on the concept of sustainability using a qualitative research approach. In chapter 7 the key determinants of sustainability with respect to early post-implementation successes of SSP and the ERAS programme for colonic surgery are studied. Perceived key determinants of sustainability are analysed three to six years following successful implementation. In chapter 8 , strategies that help, have helped or have the potential to help to sustain the ERAS programme for colonic surgery as perceived by professionals after the hospitals joined a quality improvement collaborative, are presented.

Chapter 7 explores determinants of the sustainability of SSP and the ERAS programme for colonic surgery three to six years following implementation. An explorative multiple qualitative case study was performed in which the two innovation cases were used. Semi structured interviews were conducted with 25 key persons involved in the care process. A direct content analysis was performed and Damschroder's Consolidated Framework Implementation Research (CFIR) was used as guidance during the data collection and analysis. Respondents perceived trust and the belief in the innovation, spread of the programmes to other settings, adaptability of a programme, institutionalisation of the programmes into existing systems and short communication lines within the multidisciplinary team as key determinants of sustainability. This chapter shows that sustainability is a complex process and mainly the same determinants of sustainability were put forward in both cases. The study reveals two determinants of sustainability beyond the CFIR, i.e. modification of the innovation and spread to other contexts.

Chapter 8 describes promising strategies for the sustainability of the ERAS programme for colonic surgery as perceived by professionals after their hospital joined a quality improvement collaborative (QIC). The identified strategies were targeting the professionals and the organisation. Identified activities were internal audit and feedback on outcomes, small-scale educational booster meetings, reminders, changing the physical structure of the organisation, changing the care process, making work agreements, delegating responsibility and involving a coordinator. A multifaceted self-driven strategy was executed in most hospitals and in most hospitals promising strategies were proposed to sustain the ERAS programme. This chapter suggests that post-implementation investment at the institutional level is needed to sustain the ERAS programme after a hospital joined a QIC and achieved early post-implementation success.

\section{Part IV Discussion}

Part four is the final part of the thesis (chapter 9). Chapter 9 provides an overall conclusion and the general discussion of this thesis. This chapter explains that this thesis contributed to the exploration of the concept of sustainability of health care innovations 
and shows that this sustainability research is original as it focuses on hospital-based multidisciplinary innovations. Furthermore, the research presented in this thesis shows that in both innovation cases, the level of sustainability differed between hospitals and the level of sustainability was different on sustainability indicator level. The chapter presents the methodological considerations of the research presented in this thesis. It also reveales gaps in current sustainability research and it describes starting points for further sustainability research and projects. 
Samenvatting 
Implementeren van innovaties in de zorg gaat niet vanzelf. In ziekenhuizen zijn veel personen en teams actief betrokken om de zorg te verbeteren. Deze verbeterprojecten en activiteiten zijn vooral gericht op het behalen van korte termijn effecten. Vaak is het onbekend of en in hoeverre deze kwaliteitsverbeteringen duurzaam zijn. Daarnaast is weinig onderzoek is gedaan naar de mate waarin korte termijn successen van actieve implementatie beklijven en naar de determinanten voor de duurzaamheid van succesvol geïmplementeerde zorginnovaties.

Twee innovaties, een kortverblijfprogramma voor darmchirurgie (ERAS) en een kortverblijfprogramma voor borstkankerchirurgie (SSP), werden drie tot zes jaar na succesvolle implementatie bestudeerd. Het ERAS programma werd in 33 ziekenhuizen geïmplementeerd tussen 2006 en 2009 door middel van een doorbraak implementatiestrategie. SSP werd in vier early adopter ziekenhuizen geïmplementeerd tussen 2006 en 2007. Hierbij is gebruik gemaakt van een ziekenhuis-specifieke implementatiestrategie gebruikt, waarbij de invulling van de implementatiestrategie afhankelijk was van de behoeften van de ziekenhuizen om SSP succesvol te implementeren.

Deze thesis heeft als doel het concept duurzaamheid van zorginnovaties na actieve implementatie in de ziekenhuiszorg te onderzoeken. Duurzaamheid is een nieuw begrip in de gezondheidszorg en verschillende definities en perspectieven worden in implementatieonderzoek gebruikt. Het concept duurzaamheid wordt onderzocht aan de hand van de twee implementatiecasussen. De thesis bestaat uit vier onderdelen, namelijk een introducerend deel, een deel met kwantitatieve evaluaties, een deel met kwalitatieve evaluaties en een afsluitend deel.

\section{Deel I Introductie}

Het eerste deel (hoofdstukken 1 - 2), richt zich op de achtergrond van het proefschrift. Dit onderdeel bevat de algemene inleiding, hoofdstuk 1 , en het studieprotocol van de 'SUStainability of Health care Innovations' (SUSHI) studie, hoofdstuk 2.

Hoofdstuk 1 beschrijft in een algemene inleiding, de relevantie van het onderwerp en geeft een overzicht van de belangrijkste elementen van de studie. Duurzaamheid en concepten gerelateerd aan duurzaamheid zoals innovatie, kwaliteitsverbetering en implementatie worden beschreven. Daarnaast wordt de achtergrond van de twee innovatiecasussen toegelicht. Tenslotte wordt in dit hoofdstuk het doel van het proefschrift beschreven en worden de vraagstellingen genoemd. Deze thesis onderzoekt in hoeverre innovaties zijn behouden een aantal jaar na succesvolle implementatie en onderzoekt het concept duurzaamheid van innovaties in de zorg na succesvolle implementatie middels verschillende evaluaties. 
Hoofdstuk 2 presenteert de methodologie van de SUSHI-studie. Een kwantitatieve en kwalitatieve onderzoeksmethode werd toegepast om het duurzaamheidsconcept te exploreren. Duurzaamheid van patiëntuitkomsten en duurzaamheid van de opvolging van de zorgprogramma's door de zorgprofessional werden bepaald drie tot zes jaar na succesvolle implementatie. Tevens werd de duurzaamheid van de economische waarde van SSP vanuit een maatschappelijk perspectief bepaald. Om de determinanten voor de duurzaamheid van twee innovatiecasussen te exploreren, werden daarna semigestructureerde interviews gehouden met sleutelfiguren.

\section{Deel II Kwantitatieve evaluaties over duurzaamheid}

In het tweede deel van dit proefschrift (hoofdstuk 3-6) wordt de mate van duurzaamheid van SSP en het ERAS programma bepaald vanuit een kwantitatief onderzoeksperspectief. Patiëntuitkomsten en de opvolging van de zorgprogramma's door de zorgprofessional worden onderzocht in respectievelijk hoofdstuk 3 en hoofdstuk 4 . In hoofdstuk 5 wordt het concept duurzaamheid toegepast op de economische waarde met de SSP casus ter illustratie. Het onderdeel sluit af met een systematische review over de duurzaamheid van richtlijnopvolging door professionals in de medische zorg.

Hoofdstuk 3 onderzoekt de duurzaamheid van SSP vijf jaar na implementatie. De mate van duurzaamheid van het zorgprogramma werd bepaald door het percentage patiënten dat in verkorte opname werd behandeld (dagbehandeling of 24-uursopname) te onderzoeken en door de richtlijnopvolging door zorgprofessionals in kaart te brengen. Het gemiddelde percentage borstkankerpatiënten dat in verkorte opname werd behandeld was vijf jaar na implementatie gelijk gebleven op 82\% (ziekenhuis 1: 83\%, ziekenhuis 2: $78 \%$, ziekenhuis 3: $87 \%$, ziekenhuis 4: $80 \%$ ). Dit was vergelijkbaar met de resultaten direct na implementatie van het zorgprogramma $(p=0.938)$. De gemiddelde opvolging van de zorgprogrammaonderdelen vanuit het perspectief van de zorgprofessional steeg van $65 \%$ op het moment direct na implementatie naar $78 \%$ vijf jaar na implementatie. De studie laat zien dat verkorte opname na borstkankerchirurgie was behouden in de vier ziekenhuizen en dat een borstsparende operatietechniek een voorspellende factor was voor verkorte opname (OR 95\% Cl 4.3 1.6-11.2).

Hoofdstuk 4 onderzoekt de duurzaamheid van het ERAS programma (darmchirurgie) in ziekenhuizen drie tot vijf jaar na deelname aan een doorbraakimplementatiestrategie. Uit de 33 ziekenhuizen die deelnamen aan de implementatiestudie, werden de tien ziekenhuizen geselecteerd die destijds het best hadden gepresteerd tijdens de implementiestudie. Succes was omschreven als een mediane opnameduur van zes dagen of korter en richtlijnopvolging door de professionals van minimaal $70 \%$. De gemiddelde mediane opnameduur steeg van 5,25 naar 6 dagen, deze stijging was echter niet significant. De duur tot functioneel herstel was gelijk in beide metingen: een mediaan van 3,00 dagen (interkwartielafstand P25 2,88 - P75 3,00; min. 2,00 - max. 4,00) drie 
tot vijf jaar na implementatie in vergelijking met een mediaan van 3,00 dagen (interkwartielafstand P25 3,00 - P75 3,50; min. 3,00 - max. 4,00) vlak na implementatie $(P=0.26)$. Richtlijnopvolging daalde van 75\% (SD 7.6 min. 64\% - max. 87\%) naar 67\% (SD 6.3 min. 56\% - max. 73\%) ( $p=0.052)$. Met name richtlijnopvolging van de ERASelementen die uitgevoerd moeten worden na de darmoperatie daalde. Dit hoofdstuk laat zien dat het ERAS programma redelijk was behouden in de tien ziekenhuizen. Er was variatie geconstateerd tussen ziekenhuizen en op indicatorniveau.

Hoofdstuk 5 introduceert het concept duurzaamheid in relatie tot economische waarde van een zorginnovatie. Hierbij werd gebruik gemaakt van de 'netto monetaire baten' (NMB) benadering met een maximale bereidheid tot betalen van $€ 80.000$ per QALY. Het hoofdstuk laat zien dat de economische waarde van SSP vijf jaar implementatie volledig was behouden in vergelijking met de economische waarde direct na implementatie. De gemiddelde incrementele NMB (iNMB) was $€ 1649$ per patiënt. De iNMB daalde licht vijf jaar na implementatie na correctie voor leeftijdscategorie. Vanuit een gezondheidszorgperspectief was de NMB vijf jaar na implementatie €487 hoger. De mate van duurzaamheid van de economische waarde van SSP was verschillend tussen ziekenhuizen, zowel vanuit een maatschappelijk- als vanuit een gezondheidszorgperspectief. Toepassing van de NMB benadering voor de analyse van duurzaamheid van economische waarde leidde tot verschillende discussiepunten, zoals de vraag of regressieanalyse uitgevoerd moet worden om te corrigeren voor casemixverschillen die in de loop van de tijd ontstaan zijn, het analyseniveau (vanuit een enkelvoudig ziekenhuisperspectief of vanuit een meervoudig ziekenhuisperspectief) en het gewenste moment van duurzaamheidsanalyse.

Hoofdstuk 6 presenteert een systematische review die is gericht op de duurzaamheid van het opvolgen van richtlijnen door zorgverleners. Het hoofdstuk richt zich op adherentie aan medische richtlijnen meer dan een jaar na het einde van actieve implementatie. Dertien studies voldeden aan de inclusiecriteria, die in totaal zeventien evaluaties omvatten. De gemiddelde tijdsduur tussen het eind van implementeren en de duurzaamheidsevaluatie was 2,7 jaar [min 1.5 - max 7.0]. In 41\% van de evaluaties (7 van de 17) bleek de richtlijn nog altijd opgevolgd te worden, terwijl dit niet was behouden in vijf evaluaties. Vier evaluaties presenteerde gemixte resultaten en bij één evaluatie was het onduidelijk of de richtlijnopvolging was behouden. Richtlijnopvolging was behouden op een niveau van minstens $90 \%$ in $53 \%$ (9 van de 17) van de evaluaties. De resultaten suggereren dat updates van klinische richtlijnen kunnen leiden tot terechte afwijking van de originele richtlijn. Door het beperkte aantal studies, de afwezigheid van een uniforme definitie, het hoge risico op bias en de gemixte resultaten van de studies, kan geen robuuste conclusie over de duurzaamheid van de opvolging van medische richtlijnen door zorgverleners worden getrokken. 


\section{Deel III Kwalitatieve analyses over duurzaamheid}

Het derde deel (hoofdstuk 7-8) van dit proefschrift focust op het duurzaamheidsconcept waarbij gebruik wordt gemaakt van een kwalitatieve onderzoeksmethode. In hoofdstuk 7 worden de determinanten voor de duurzaamheid van de succesvol geïmplementeerde innovaties SSP en het ERAS programma geanalyseerd. Determinanten die door zorgverleners worden ervaren als bevorderend dan wel belemmerend werden drie tot zes jaar na implementatie bestudeerd. In hoofdstuk 8 worden activiteiten gepresenteerd die waardevol waren of zouden kunnen zijn voor de duurzaamheid van het ERAS programma na de toepassing van een doorbraakimplementatiestrategie.

Hoofdstuk 7 heeft als doel de determinanten voor duurzaamheid van SSP en het ERAS programma drie tot zes jaar na implementatie te exploreren. Semi-gestructureerde interviews werden afgenomen met 25 sleutelfiguren zoals chirurgen, verpleegkundig specialisten en afdelingshoofden die betrokken waren bij SSP of het ERAS programma. Het 'Consolidated Framework Implementation Research' (CFIR) van Damschroder werd gebruikt tijdens de dataverzameling en data-analyse. Determinanten die volgens de respondenten relevant waren voor de duurzaamheid van het ERAS programma en SSP waren: vertrouwen en geloof in de innovatie, verspreiding van de innovatie naar andere contexten, de mogelijkheid om de innovatie in de lokale context aan te passen, de mogelijkheid om de innovatie te modificeren, institutionalisatie van de innovatie en korte communicatielijnen binnen het multidisciplinaire team. Het hoofdstuk laat zien dat de duurzaamheid van een zorginnovatie een complex proces is. Daarnaast laat het onderzoek zien dat dezelfde determinanten van toepassing zijn in beide casussen.

In hoofdstuk 8 worden mogelijke faciliterende activiteiten onderzocht voor het behoud van het ERAS programma nadat een ziekenhuis heeft deelgenomen aan een doorbraakimplementatiestrategie. De resultaten laten zien dat activiteiten gerelateerd aan de zorgverlener en de organisatie worden ervaren als faciliterend voor de duurzaamheid van ERAS. Activiteiten gerelateerd aan de zorgprofessional zijn: interne audit en terugkoppeling van resultaten, kleinschalige educatiebijeenkomsten om kennis op te frissen, herinneringen, aanpassing van de organisatiestructuur, verandering van het zorgproces, werkafspraken maken, verantwoordelijkheid delegeren en het aanstellen van een coördinator. In ieder ziekenhuis werd ervaren dat een strategie bestaande uit meerdere activiteitsoorten potentie had om een innovatie te waarborgen in de periode na succesvolle implementatie. Het hoofdstuk concludeert dat deze activiteiten waardevol kunnen zijn voor de duurzaamheid van het ERAS-programma na uitvoering van een doorbraakimplementatiestrategie. 


\section{Deel IV Discussie}

Onderdeel vier bevat de algemene discussie van het proefschrift (hoofdstuk 9).

Dit hoofdstuk presenteert de algemene conclusie van deze thesis en geeft een algemene discussie. Het hoofdstuk laat zien dat deze thesis bijdraagt aan de exploratie van het concept duurzaamheid van zorginnovaties na succesvolle implementatie. Daarnaast laat dit hoofdstuk zien dat het onderzoek origineel is binnen het huidige onderzoek omdat het zich richt op innovaties in de multidisciplinaire ziekenhuiswereld. De resultaten in dit proefschrift laten zien dat de lange termijn resultaten van beide implementatiestudies verschillend waren op ziekenhuisniveau en op indicatorniveau. Het hoofdstuk gaat in op de methodologie van het onderzoek en beschrijft tenslotte de richting waar toekomstig onderzoek en toekomstige projecten zich op kunnen focussen. 
Valorisation 
Valorisation of knowledge is a process of making knowledge available for society. Besides educating students and performing research, universities play a major role in the valorisation of new obtained insights. Although a lot of scientific research is performed in the Netherlands, the actual uptake of knowledge in society is limited. The current chapter describes the valorisation potentials of this thesis.

The present thesis explored the concept of sustainability of health care innovations in hospital care after active implemention of these innovations. The findings that are reported in this thesis can be of relevance for health care professionals, patients, researchers, quality improvement specialists, policy makers and insurance companies. The valorisation possibilities are described on target group level.

\section{Healthcare professionals}

For results of research to be effective in practice, they have to be communicated to health care professionals. The research results presented in this thesis were presented at several international and national conferences aimed at general surgeons and breast cancer and gastroenteral surgeons. The results were also presented to other disciplines. For example, the ERAS programme for gynaecologic surgery was implemented in 15 hospitals in 2015. The findings regarding the sustainability of the ERAS programme for colonic surgery were shared at a gynaecologic symposium targeting the hospitals involved with the implementation project. The insights of this thesis were useful to motivate and facilitate sustainable change in the gynaecologic setting.

\section{Patients}

Yearly, many patients receive medical care in the Netherlands. Unfortunately, a major proportion of these patients do not receive care following the latest evidence, which may lead to negative results on morbidity and mortality. Besides harm to patients, health care costs may increase as a result of imperfect care delivery. Sustainability of actively implemented innovations potentially leads to a reduction in medical errors. Reduction of medical errors will lead to increased quality of care and a reduction in unnecessary care or care as a result of complications and therefore in costs. The results of this thesis were communicated to care givers involved in the breast cancer surgery and colonic surgery setting. As a result of the valorisation of the findings presented in this thesis, professionals may have adjusted their long-term routines with respect to the ERAS programme or the short stay programme years after successful implementation. The impact of this research in terms of sustainability of medical innovations can be valuable for breast cancer and colonic cancer patients. As the ERAS programma was 
implemented in 15 hospitals in the Netherlands between 2014 and 2015, the insights of this thesis can also be valuable for gynecologic patients.

\section{Scientific community}

The results of this thesis contribute to current models for determinants of effective implementation and cost-effectiveness. The results and recommendations of this thesis were published in peer reviewed international journals and were presented at international scientific conferences. The journals and conferences had a quality improvement, surgical or a health technology assessment scope. Furthermore, this thesis will be spread among researchers in the main researcher's network. These researchers are specialised merely in quality improvement, surgery or health technology assessment and they may use these insights in their research. The paper "Identification of promising strategies to sustain improvements in hospital practice" (BMC Health Services Research 2014, 14:641) was mentioned as an editor's choice on the homepage of BMC Health Services Research. This may have facilitated the valorisation of the knowledge to this target group.

Also, data of the Netherlands Cancer Registry was used in the discussion of chapter 3 of this thesis. The Netherlands Cancer Registry has the policy to collect publications if their data is used. As a result, this generates structural valorisation between research parties. This thesis was also send to the Netherlands Cancer Registry.

\section{Quality improvement experts}

Last decades, a large body of research evidence has become available and from this, new technologies and ideas for process redesigns have emerged. The rate of scientific publications has been increasing over the last 50 years and new insights are increasingly being spread using new distribution channels such as open access journals and publications on the internet. Given the exponential increase in knowledge on innovations and knowledge on effective implementation strategies to implement and sustain these innovations, the actual use of the last available evidence has become quite a challenge. There is a growing interest in clinical practice guidelines and they are increasingly being developed and implemented in practice. Although the goal of implementation projects is to embed health care innovations in daily routines, many implementation projects focus primarily on immediate results. To create awareness about the long-term impact of quality improvement efforts, the current thesis was sent to the Dutch institute for healthcare improvement ( $\mathrm{CBO}$, Kwaliteitsinstituut voor de gezondheidszorg $\mathrm{CBO}$ ) and to the Netherlands organisation of health research and development (ZonMw) implementation fellows. The implementation fellows aim to transfer knowledge about implemen- 
tation of evidence in hospitals. Also, they support implementation research proposals and projects. The research presented in this thesis will be discussed during next implementation fellow meeting.

\section{Policymakers}

Most research that is performed in the Netherlands is financially supported by external organisations. In health care for example, ZonMw invests in health research. The thesis revealed gaps in current sustainability research and the insights of this thesis may be a starting point for further sustainability research and projects related to healthcare.

An interview was given for the ZonMw journal Mediator (December 2014, issue 9). Mediator is a journal with articles about results and updates of ZonMw quality improvement projects. The journal is digital and is spread among all people involved in ZonMw quality improvement projects and to people who subscribed to receive this journal. The subject of the interview was the sustainability of the short stay programme for breast cancer surgery. In this interview results and recommendations for practice were given. Hopefully, this interview and this thesis created awareness about the need for sustainability of evidence-based health care innovations in the real-world to safeguard the time and money invested to improve medical care. To ensure that policy makers are aware of the potential of a sustainability approach, this thesis was distributed among the innovation departments of policy makers such as ZonMw and the Dutch Ministry of public health, welfare and sports.

\section{Health insurance companies}

Increasingly, insurance companies aim to contract integrated patient centred care. The current thesis revealed that an integrated care prorgamme for breast cancer surgery patients was sustained five years following implementation in four early adopter hospitals. This programme is a good illustration of structural and integrated primary and secondary care collaboration. Treating patients in short stay was shown to be effective, safe, efficient and sustainable. Insurance companies could use the insights of this thesis in their contracting policy for oncologic care. 
Dankwoord 
Allereerst wil ik de borstkankerpatiënten bedanken die hebben deelgenomen aan de SUSHI-studie. Het invullen van de vragenlijsten en het kostenboekje, tijdens een uiterst moeilijke periode in het leven, is van ongekend belang geweest voor het uitvoeren van deze studie. Zonder uw deelname had ik het kortverblijfprogramma voor borstkankerchirurgie niet in deze vorm kunnen analyseren.

Naast dat het promotieonderzoek erg interessant was, had ik gelukkig ook te maken met een warm en breed georiënteerd promotieteam. Tijdens het sollicitatiegesprek voelde ik direct de brede expertise, connectie, motivatie en het vertrouwen. Het promotieteam bestond uit een implementatie-expert, een expert op het gebied van kwaliteitsverbetering, een gezondheidseconoom, een oncologisch chirurg, een artsonderzoeker en een gezondheidswetenschapper. Deze samenstelling bleek een zeer goede combinatie te zijn voor de uitvoering van dit onderzoeksproject.

Prof. dr. Van der Weijden, Trudy, hartelijk dank voor de mogelijkheid die je me hebt gegeven om dit onderzoek uit te voeren. Voor iedereen was 'sustainability' een nieuw concept dat tot vele discussies leidde. Jij wist, dankzij de implementatiekennis, doorslaggevend advies te geven. Daarnaast gaf je me alle ruimte en mogelijkheden om me te ontwikkelen tijdens deze promotiereis. Heel veel succes gewenst met de ontwikkeling van het onderzoeksprogramma promoting health and personalized care!

Prof. dr. Von Meyenfeldt, Maarten, ontzettend bedankt dat je ook na het verlaten van de afdeling chirurgie, het voorzitterschap bij de METC, en andere prioriteiten, daadkrachtig doorging met het leiden van dit project. Naast de klinische ervaring verdiep je je ook al jaren in de aspecten rondom de chirurgie. Of het nou gaat om klinische vraagstukken, zorgmanagementzaken, zorgvernieuwingen of medisch ethische kwesties; deze aspecten combineer jij als 'zorg-allrounder'. Deze inzichten waren zeer bruikbaar bij de uitvoering van dit onderzoek. Vergeet ook niet van het pensioen te genieten, zeker na dit wetenschappelijke huwelijk.

Prof. dr. Dirksen, Carmen, enorm bedankt voor de waardevolle brainstormsessies en de pientere feedback op papers. Deze 'Ament' heb je met vol enthousiasme begeleid en je ging tot het uiterste. Jouw focus, kritische feedback en de 'open minded' blik op sustainability gerelateerde vraagstukken hebben enorm bijgedragen aan de basis van dit eindresultaat. Als professor Health Technology Assessment of clinical interventions en afdelingshoofd bij KEMTA wens ik je heel veel succes toe.

Dr. Maessen, José, ook wil ik jou ontzettend bedanken voor de tijd die je in de manuscripten hebt gestoken. Als ERAS-expert, implementationfellow en kwaliteit- en veiligheidsspecialist heb je me ontzettend veel geleerd. Zelfs als de agenda het eigenlijk niet toeliet, maakte je tijd vrij om inhoudelijke input te geven. De 'city hopper' tripjes naar Cannes en Londen waren door jouw aanwezigheid extra gezellig. Enorm veel succes gewenst bij de ontwikkeling van 'het ziekenhuis van de toekomst' en met de uitrol (en duurzaamheid) van ERAS gynaecologie in Nederland. 
Freek, co-PhDer en sinds kort weer stadsgenoot, dank voor de samenwerking en de gezelligheid tijdens dit onderzoeksproject. Van Antwerpen tot Stadskanaal (via NieuwAmsterdam), waar zijn we niet geweest om data te verzamelen of een cursus te volgen. Ik ben benieuwd of de dagelijkse 'lachgolf' vanuit onze werkkamer op de afdeling KEMTA gemist wordt. Ontzettend veel succes bij de chirurgie in het Medisch Spectrum Alkmaar! We moeten maar snel in wat SUSHI snijden en wat bubbels drinken om onze boekjes te vieren.

Ook wil ik de leden van de beoordelingscommissie hartelijk danken voor het beoordelen van mijn proefschrift: prof. dr. L.W. van Rhijn, prof. dr. G.L. Beets, em. prof. dr. R. Grol, prof. dr. H.L. Severens en dr. H. Fiolet.

De deelname van de veertien participerende ziekenhuizen aan de studie is van uiterst belang geweest. Ik wil daarom alle betrokkenen en respondenten van de ziekenhuizen bedanken die hebben bijgedragen aan deze studie. Voor de SSP (MaDO) ziekenhuizen was de deelname aan de studie omvangrijker en intensiever. Ik ben me zeer bewust van het feit dat ik zonder de medewerking en de support van de artsen, (bovenal) de verpleegkundig specialisten, physician assistants, mammacare verpleegkundigen en afdelingshoofden het onderzoek niet had kunnen uitvoeren.

Ook de co-auteurs ben ik dankbaar voor de inspirerende en kritische bijdragen aan de papers. Jeanny de Groot, review buddy, wat heeft onze team spirit geresulteerd in een mooie, vlotte en succesvolle samenwerking. Heel veel succes met de afronding van jouw promotieonderzoek en de toekomstige baan bij de gynaecologie. Ik hoop dat we deze gezelligheidsmomenten kunnen blijven herhalen (maar dan ook zonder de uitvoering van een review). Dr. de Kok, beste Mascha, bedankt voor de antwoorden op MaDOgerelateerde vragen, die verspreid over afgelopen jaren, aan je werden gesteld. Zonder jouw geinvesteerde bloed, zweet en tranen in implementatiestudie kon ik deze studie niet uitvoeren. Veel succes gewenst als huisarts in spe. Prof. dr. Kleijnen, Jos, hartelijk dank voor het methodologische advies bij de uitvoering van de systematic review. Prof dr. Grimshaw, Jeremy, thank you for advising me about the discussion of the systematic review during the master class you gave at Maastricht University in 2014. Dr. Moser, Albine, jij hebt me telkens geholpen als ik vragen had over kwalitatief onderzoek waarvoor dank. Ik heb door jou veel geleerd over de uitvoering en het schrijven over kwalitatief onderzoek.

Karin Vaessen, er leek maar geen einde te komen aan de op te vragen papers voor de review. Gelukkig hielp je me bij het verzamelen van de papers voor de systematic review en stond je ook bij andere verzoeken altijd voor me klaar waarvoor dank. 
Fons, hartelijk dank voor het advies over de statistiek en voor de gezonde streekproductproeverijen! Geniet van je welverdiende pensioen.

Collega's op de afdeling KEMTA, dank voor de gezellige lunches, de praatjes bij de koffiecorner; kortom de gezellige tijd! De theetjes en koffietjes waren ook enorm waardevol om te reflecteren als onderzoeker. Dank voor de lunches en koffiemomenten waarbij ervaringen over onderzoek, publicatieplannen ("anders kun je ook in de VIVA publiceren") en het regelen van de promotie werden gedeeld.

Van elke patiënt met kanker verzamelt het Integraal Kankercentrum Nederland (IKNL) data in de Nederlandse Kankerregistratie (NKR). Ik dank de registratieteams van IKNL voor de dataverzameling in de NKR en de wetenschappelijke medewerkers voor de analyse en aanlevering van data uit de NKR die gebruikt zijn in dit proefschrift.

Wat moet een promovendus zonder paranimfen. Gelukkig word ik ondersteund en vergezeld door twee sterke vrouwen (en die heb ik nodig bij de mannelijke beoordelingscommissie;-)): Inge en Marianne. Marianne, wat was je een fijne kamergenoot en wat maakte je overheerlijke (chocolade) baksels die ik ook op voorgenomen balansdagen zeker niet kon afslaan (ik mis ze!). Je bent een geweldige collega en moeder en ik wens je heel veel succes in de toekomst! Inge, lieve vriendin, twee jaar geleden had ik je al gevraagd (of jij mij?) om paranimf te zijn. Voortvarend sta jij meteen in de actiemodus om me te begeleiden naar DE dag en dat waardeer ik enorm. Ook hebben wij hebben een andere SUSHI-historie achter ons (dank Faye en Dominique!). Dat de receptie bij Ginger wordt gehouden is daarom geen toeval! Bedankt voor alle steun en gezellige momenten!

Veel vrienden en familieleden hebben interesse getoond en me gesupport tijdens mijn promotieonderzoek en het schrijven van dit proefschrift. Ik wil enkelen daarvan in het bijzonder benoemen. Stefanie, wat mis ik de gezellige lunches in de Mensa met je. Gelukkig zijn we goed in improviseren met onze tijdbesteding en spreken we elkaar regelmatig. Veel succes bij Medtronic en veel geluk gewenst met je gezin. Eem, bedankt voor de interesse in het onderzoek. Jij gaf me inspirerende input en energie voor het uitvoeren van dit onderzoek. Hopelijk organiseer je samen met je man snel een housewarming in Nijmegen! Lieve Farah en Bernhard, bedankt voor jullie support en de gezellige zonnige weekenden met heerlijk eten in Scheveningen. Arnold, Caroline en Jack, veel dank voor de continue steun en de interesse voor de voortgang van het onderzoek.

Mama en Bo, bedankt voor alle liefde en steun! Wat hebben we de afronding van de promotie goed gevierd in Rome! Zusje, ondanks dat we zo van elkaar verschillen zie ik steeds vaker dat we op elkaar lijken. Ik ben heel blij met jou als mijn zus, een leven zonder jou is ondenkbaar. Mama, bedankt voor de vrijheid die je me hebt gegeven om 
me te ontwikkelen tot wie ik ben. Het is fijn om na een werkweek te kunnen crashen in het knusse Zuiden, al is het ontdekken van Amsterdam met jullie ook altijd een feest! Ik hou van jullie!

Homam, liefde van mijn leven, het proefschrift is af! YES, dat betekent meer vrije tijd in het weekend! Jouw eerlijkheid en afstand van de academische wereld hielpen mij enorm om zaken te relativeren. Ik hoop dat we samen een mooi, lang en gezond leven tegemoet gaan. Ik hou van je en ik ben dankbaar dat wij duurzaam zijn.

Stephanie 

About the author 


\section{About the author}

Stephanie Ament was born in Heerlen on January 30, 1986. From 2005 till 2008 she attained her bachelor degree in Health Sciences majoring in policy and management at Maastricht University. After finishing her bachelor she completed her master degree in Health Care Management at the Erasmus University Rotterdam in 2009. Her master thesis concentrated on the optimalisation of Maastro Clinic's head throat cancer pathway using lean thinking.

From May 2009 until April 2011 she was consulent finance and registration at the department Finance and Information of University Hospital Maastricht. From April 2011 until December 2014 she worked on her PhD research project at the department KEMTA, at the Maastricht University Medical Centre (MUMC) and was employed at the School for Public Health and Primary Care (CAPHRI, UM). The 'SUStainability of HealthCare Innovations' (SUSHI) research project was financially supported by ZonMw (project number 171103004). The aim of the SUSHI study was to explore the concept of sustainability of innovations in hospital practice after successful implementation. Currently, Stephanie is employed as a specialist at the department of compliance (health care purchase) at Zilveren Kruis in Leusden.

E-mail:

Linkedln \& Twitter: Research gate: stephanieament@gmail.com

stephanieament

stephanie_mc_ament 


\section{List of publications and presentations}

\section{Peer-reviewed publications}

Stephanie M C Ament, Freek Gillissen, José M C Maessen, Carmen D Dirksen, Trudy van der Weijden, Maarten F von Meyenfeldt. Sustainability of health care innovations (SUSHI): long-term effects of two implemented surgical care programmes (protocol) BMC Health Services Research 2012, 12:423

Stephanie M C Ament, Freek Gillissen, José M C Maessen, Carmen D Dirksen, Toine V R J Bell, Yvonne L J Vissers, Trudy van der Weijden, Maarten F von Meyenfeldt. Sustainability of short stay for breast cancer surgery in early adopter hospitals The Breast, Volume(s) 23, 01-april-2014, Pages 429-434

Freek Gillissen, Stephanie M C Ament, José M C Maessen, Cees H C Dejong, Carmen D Dirksen, Trudy van der Weijden, Maarten F von Meyenfeldt. Sustainability of an Enhanced Recovery After Surgery programme in colonic surgery World Journal of Surgery 2015 Feb;39(2):526-33

Stephanie M C Ament, Freek Gillissen, Albine R N Moser, José M C Maessen, Carmen D Dirksen, Trudy van der Weijden, Maarten F von Meyenfeldt. Identification of promising strategies to sustain improvements in hospital practice BMC Health Services Research $2014,14: 641$

Stephanie M C Ament, Mascha de Kok, Cornelius J H van de Velde, Jan A Roukema, Toine V R J Bell, Fred W van der Ent, Trudy van der Weijden, Maarten F von Meyenfeldt, Carmen $D$ Dirksen. A detailed report of the resource use and costs associated with implementation of a short stay programme for breast cancer surgery Implementation Science 2015, 10:78

\section{Submitted publications}

Stephanie M C Ament, Freek Gillissen, Albine R N Moser, José M C Maessen, Carmen D Dirksen, Maarten F von Meyenfeldt, Trudy van der Weijden. What determines sustainability of two multidisciplinary hospital based programmes after achieving early implementation success? A qualitative study Submitted 
Stephanie M C Ament, Jeanny J de Groot, José M Maessen, Carmen D Dirksen, Trudy van der Weijden, Jos Kleijnen. Sustainability of professionals' adherence to guideline recommendations in medical practice: a systematic review Submitted

Stephanie M C Ament, Freek Gillissen, José M C Maessen, Jan A Roukema, Maarten F von Meyenfeldt, Carmen D Dirksen. Sustainability of value of a short stay programme for breast cancer surgery care five years after implementation Submitted

Jeanny J de Groot, Stephanie M C Ament, Jos Kleijnen, José M Maessen, Brigitte F Slangen. What are the benefits of Enhanced Recovery After Surgery (ERAS) programmes for patients undergoing abdominal gynecologic surgery? A systematic review Submitted

Freek Gillissen, Stephanie M C Ament, José M C Maessen, Carmen D Dirksen, Trudy van der Weijden, Maarten F von Meyenfeldt. An ongoing gap between functional recovery and discharge after colonic surgery: a qualitative case study exploring barriers and facilitators of early discharge Submitted

\section{Presentations}

Stephanie M C Ament, Freek Gillissen, José M C Maessen, Carmen D Dirksen, Trudy van der Weijden, Maarten F von Meyenfeldt. Is the ERAS programme normalised? Sustainability of the 'Enhanced Recovery After Surgery' programme in colonic surgery after using a breakthrough implementation strategy ERAS congress 2012, Cannes (France). Poster presentation

Stephanie M C Ament, Freek Gillissen, José M C Maessen, Carmen D Dirksen, Trudy van der Weijden, Maarten F von Meyenfeldt. Sustainability of healthcare innovations in medical care. PhD Forum at Jagiellonian University Medical College 2012, Krakow (Poland). Oral presentation

Stephanie M C Ament, Freek Gillissen, José M C Maessen, Carmen D Dirksen, Trudy van der Weijden, Maarten F von Meyenfeldt. How to sustain implementation successes? Quality and Safety forum 2012, London (England). Poster presentation

Freek Gillissen, Stephanie M C Ament, José M C Maessen, Carmen D Dirksen, Trudy van der Weijden, Maarten F von Meyenfeldt. Sustainability of a short stay programme after breast cancer surgery in four early adopting hospitals. European Cancer Congress (ECCO) 2013, Amsterdam (the Netherlands). European Journal of Cancer, Volume 49 Supplement 2, September 2013. Poster presentation 
Freek Gillissen, Stephanie M C Ament, José M C Maessen, Carmen D Dirksen, Trudy van der Weijden, Maarten F von Meyenfeldt. Sustainability of an enhanced recovery programme in colonic surgery in the Netherlands. European Cancer Congress (ECCO) 2013, Amsterdam (the Netherlands). European Journal of Cancer, Volume 49 Supplement 2 September 2013. Poster presentation

Freek Gillissen, Stephanie M C Ament, José M C Maessen, Carmen D Dirksen, Trudy van der Weijden, Maarten $\mathrm{F}$ von Meyenfeldt. Sustainability of the ERAS programme for colonic surgery. ERAS congress 2014, Valencia (Spain). Oral abstract presentation

Stephanie M C Ament, Freek Gillissen, José M C Maessen, Carmen D Dirksen, Trudy van der Weijden, Maarten F von Meyenfeldt. Sustainability of economic value of a short stay programme for breast cancer surgery care five years following implementation The Low Lands Health Economists' Study Group (LolaHesG) 2014, Oostvoorne (the Netherlands). Paper presentation

Stephanie M C Ament, Freek Gillissen, José M C Maessen, J A Roukema, Trudy van der Weijden, Maarten F von Meyenfeldt, Carmen D Dirksen. Towards a framework for analysing sustainability of economic value: the case of a short stay programme for breast cancer surgery care five years after implementation. International Society For Pharmacoeconomics and Outcomes Research (ISPOR) 2014, Amsterdam (the Netherlands) Value in Health (2014), volume 17, issue 7, pages A662-A663. Poster presentation 